PROPERTIES AND CYCLE PERFORMANCE OF REFRIGERANT BLENDS OPERATING NEAR AND ABOVE THE REFRIGERANT CRITICAL POINT

Task 1: Refrigerant Properties

Final Report

Date Published - August, 2002

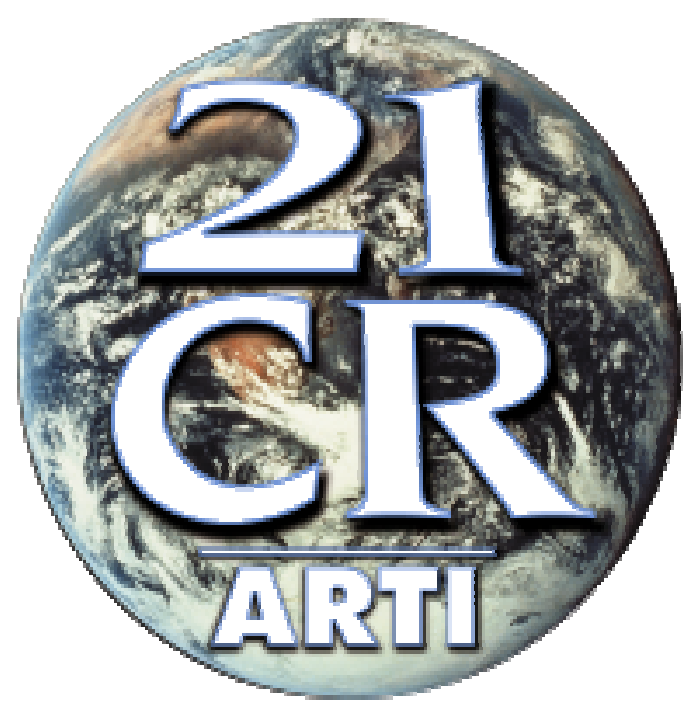

Mark O. McLinden

Arno Laesecke

Eric W. Lemmon

Joseph W. Magee

Richard A. Perkins

NATIONAL INSTITUTE OF STANDARDS AND TECHNOLOGY

Physical and Chemical Properties Division

Boulder, Colorado 80305-3328

Prepared for the

AIR-CONDITIONING AND REFRIGERATION TECHNOLOGY INSTITUTE

4100 N. Fairfax Drive, Suite 200, Arlington, Virginia 22203

Distribution A - Approved for public release; further dissemination unlimited. 


\section{DISCLAIMER}

This report was prepared as an account of work sponsored by the Air-Conditioning and Refrigeration Technology Institute (ARTI) under its "HVAC\&R Research for the $21^{\text {st }}$ Century" (21-CR) program. Neither ARTI, the financial supporters of the 21-CR program, or any agency thereof, nor any of their employees, contractors, subcontractors or employees thereof - makes any warranty, expressed or implied; assumes any legal liability or responsibility for the accuracy, completeness, any third party's use of, or the results of such use of any information, apparatus, product, or process disclosed in this report; or represents that its use would not infringe privately owned rights. Reference herein to any specific commercial product, process, or service by trade name, trademark, manufacturer, or otherwise, does not necessarily constitute nor imply its endorsement, recommendation, or favoring by ARTI, its sponsors, or any agency thereof or their contractors or subcontractors. The views and opinions of authors expressed herein do not necessarily state or reflect those of ARTI, the 21-CR program sponsors, or any agency thereof.

Funding for the 21-CR program provided by (listed in order of support magnitude):

- U.S. Department of Energy (DOE Cooperative Agreement No. DE-FC05-99OR22674)

- Air-Conditioning \& Refrigeration Institute (ARI)

- Copper Development Association (CDA)

- New York State Energy Research and Development Authority (NYSERDA)

- Refrigeration Service Engineers Society (RSES)

- Heating, Refrigeration Air-Conditioning Institute of Canada (HRAI)

Available to the public from

U.S. Department of Commerce

National Technical Information Service

5285 Port Royal Road

Springfield, VA 22161

(703) 487-4650

Available to U.S. Department of Energy and its contractors in paper from

U.S. Department of Energy

Office of Scientific and Technical Information

P.O. Box 62

Oak Ridge, TN 37831

(423) 576-8401 
ARTI-21CR/605-50010-01

DE-AI01-97EE23775

\title{
PROPERTIES AND CYCLE PERFORMANCE OF REFRIGERANT BLENDS OPERATING NEAR AND ABOVE THE REFRIGERANT CRITICAL POINT
}

\section{Task 1. REFRIGERANT PROPERTIES}

Final Report

\author{
Mark O. McLinden \\ Arno Laesecke \\ Eric W. Lemmon \\ Joseph W. Magee \\ Richard A. Perkins
}

Physical and Chemical Properties Division National Institute of Standards and Technology

325 Broadway, Mailstop 838

Boulder, CO 80303-3328

$$
\text { prepared for }
$$

Air-Conditioning and Refrigeration Technology Institute

4100 N. Fairfax Drive, Suite 200

Arlington, VA 22203

and

Building Equipment Division Office of Building Technologies

U.S. Department of Energy

1000 Independence Avenue, SW

Washington, DC 20585

August 1, 2002 


\section{EXECUTIVE SUMMARY}

This project has measured data and developed models for the properties of R410A and other HFC blends. An emphasis was placed on conditions near and above the mixture critical point-conditions where existing data were scarce and where a serious degradation in the performance of air-conditioning equipment has been observed. Many of the HFC blends have much lower critical temperatures than the CFCs or HCFCs (primarily R22) they are replacing, meaning that the operating temperatures of equipment using the blends can approach the critical temperature in extreme ambient conditions.

In this project the isochoric (constant volume) heat capacity of R125 was measured and a comprehensive, high-accuracy equation of state for the thermodynamic properties of this fluid was developed. R125 is a component of several important HFC blends, and improvements to the pure fluid formulation were a precursor to an improved mixture model. The isochoric heat capacity and pressure-density-temperature (P- $\rho-T)$ behavior of R410A were measured and these data, along with data from the literature, were used to develop a new equation of state applicable to the entire class of HFC blends. Finally, measurements of the viscosity and thermal conductivity of R410A and R507A were carried out and the resulting data were compared to the mixture model in the NIST REFPROP database. R507A is the blend R125/143a (50/50); it is a replacement for R502. The R125/143a binary pair also comprises the majority of R404A, which is the blend R125/143a/134a (44/52/4).

In support of the R125 equation of state development, the isochoric heat capacity of R125 was measured over the temperature range $305-397 \mathrm{~K}\left(32-124{ }^{\circ} \mathrm{C}\right)$ at pressures up to $20 \mathrm{MPa}$. The measurements overlapped with existing data at lower temperatures and extended well above the R125 critical point of $339 \mathrm{~K}$. These data are presented.

A new formulation is presented for the thermodynamic properties of R125 based on both literature data and the new data measured in this project. This equation of state formulation is explicit in the Helmholtz energy and can be used for the calculation of all the thermodynamic properties, including density, heat capacity, speed of sound, enthalpy, entropy, and saturation properties. Ancillary equations are given for the ideal gas heat capacity, the vapor pressure, and for the saturated liquid and vapor densities. To minimize the number of terms, the equation was developed using new non-linear fitting techniques. Comparisons to the experimental data are given to establish the accuracy of properties calculated using the equation of state. The equation of state generally represents the experimental data within their uncertainties. The estimated uncertainties of calculated values are $0.1 \%$ in density, $0.5 \%$ in heat capacity, $0.02 \%$ in speed of sound for the vapor at pressures less than $1 \mathrm{MPa}, 0.5 \%$ in the speed of sound elsewhere, and $0.1 \%$ in vapor pressure. Deviations are higher in the critical region. The equation is valid for temperatures from the triple point temperature $\left(172.52 \mathrm{~K},-100.63{ }^{\circ} \mathrm{C}\right)$ to $500 \mathrm{~K}\left(227{ }^{\circ} \mathrm{C}\right)$ and pressures up to $60 \mathrm{MPa}$.

Measurements of the P- $\rho-\mathrm{T}$ behavior of R410A were completed along 14 isochores (lines of constant density) and covered the temperature range of $200-400 \mathrm{~K}$ (-73 to $127{ }^{\circ} \mathrm{C}$ ) with pressures to $35 \mathrm{MPa}$. The isochoric heat capacity was measured along eight isochores with temperatures ranging from 303 to $397 \mathrm{~K}$ (30 to $124{ }^{\circ} \mathrm{C}$ ) with pressures up to $18 \mathrm{MPa}$. These calorimetric measurements also provided simultaneous data of the P- $\rho-\mathrm{T}$ behavior. These data are presented.

Mixture models explicit in Helmholtz energy have been developed to calculate the thermodynamic properties of HFC refrigerant mixtures containing R32, R125, R134a, R143a, and/or R152a. The Helmholtz energy of the mixture is the sum of the ideal gas contribution, the compressibility (or real gas) contribution, and the contribution from mixing. The independent 
variables are the temperature, density, and composition. The model may be used to calculate all the thermodynamic properties of these mixtures, including dew and bubble point properties and critical points. It incorporates the most accurate equations of state for each pure fluid. The form of the model is the same for all the blends considered, but blend-specific mixing functions are required for the blends $\mathrm{R} 32 / 125$ (R410) and R32/134a (a constituent binary of R407). The systems R125/134a, R125/143a, R134a/143a, and R134a/152a share a common, generalized mixing function. The estimated uncertainties of calculated properties are $0.1 \%$ in density and $0.5 \%$ in heat capacities and the speed of sound. Calculated bubble point pressures are generally accurate to within $0.5 \%$. The model is valid from 200 to $450 \mathrm{~K}\left(-73\right.$ to $\left.177{ }^{\circ} \mathrm{C}\right)$ with pressures up to $60 \mathrm{MPa}$ as verified by experimental data. The equation was developed primarily using data for binary (two-component) blends, but it is accurate in calculating the properties of the two mixtures with three constituents for which data were available. It is expected that this result will apply to other ternary and higher-order systems as well.

The new equation of state for R125 is believed to be the most accurate and comprehensive formulation for the properties of that fluid. Likewise, the mixture model developed in this work is the new state of the art for the thermodynamic properties of HFC blends. These models will be incorporated into version 7 of the NIST REFPROP database to be released in the summer of 2002.

Measurements of the viscosity and thermal conductivity of the HFC blends R410A and R507A were completed. The thermal conductivity measurements were carried out along six isotherms over the temperature range 301 to $404 \mathrm{~K}\left(28\right.$ to $\left.131{ }^{\circ} \mathrm{C}\right)$ with pressures to $38 \mathrm{MPa}$. The measurements included liquid, vapor, and supercritical states. The viscosity was measured along five isotherms over the temperature range 301 to $421 \mathrm{~K}\left(28\right.$ to $\left.148{ }^{\circ} \mathrm{C}\right)$ with pressures to $82 \mathrm{MPa}$. The originally planned viscosity measurements were also supplemented by measurements of the saturated liquid viscosity of R410A in a capillary viscometer over the range 288 to $320 \mathrm{~K}$ (15 to $\left.47{ }^{\circ} \mathrm{C}\right)$. These data were used to assess the current models for the transport properties of mixtures. These comparisons reveal that further development and refinement of the transport models is warranted. 


\section{ACKNOWLEDGEMENTS}

This work was sponsored by the Air-Conditioning and Refrigeration Technology Institute (ARTI) under their 21-CR program, the Building Equipment Division of the U.S. Department of Energy under contract number DE-AI01-97EE23775, and the National Institute of Standards and Technology (NIST).

We thank Ralph Miller of the DuPont Company for his assistance in obtaining the high-purity R32 and R125 used in this work. We thank Honeywell, Inc. for the donation of the R143a sample.

Richard Jacobsen of the Idaho National Engineering and Environmental Laboratory provided valuable assistance in the development of the R125 equation of state and the mixture model. The authors would like to acknowledge helpful discussions with Marcia Huber. We thank Tom Bruno for the analyses of the experimental samples used in this work. Mike Hiza prepared the mixtures used in the measurements. Dwain Diller assisted with the P- $\rho-T$ measurements on R410A. We also thank Piotr Domanski and Keith Rice, principal investigators of the other two tasks associated with this project, for their interactions and comments and also for providing the motivation for this work. 


\section{TABLE OF CONTENTS}

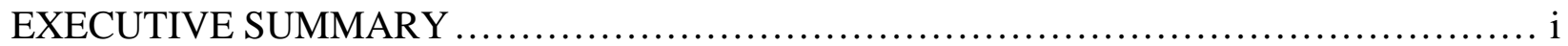

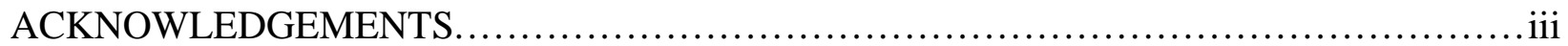

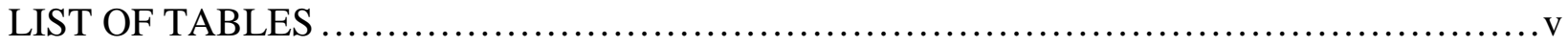

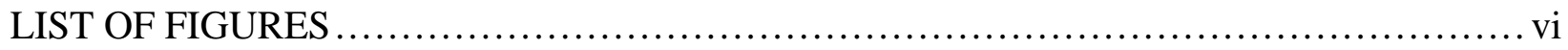

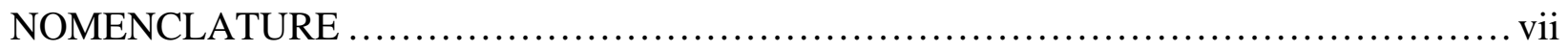

1. INTRODUCTION AND TASK STATEMENTS...................................

2. R125 HEAT CAPACITY MEASUREMENTS ........................................

3. R125 EQUATION OF STATE .............................................. 10

4. MIXTURE P- $\rho$-T AND HEAT CAPACITY MEASUREMENTS $\ldots \ldots \ldots \ldots \ldots \ldots \ldots \ldots \ldots . \ldots \ldots$

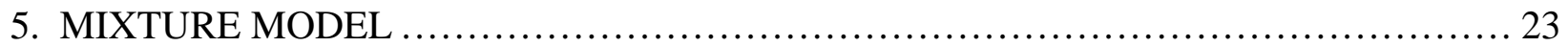

6. MIXTURE THERMAL CONDUCTIVITY MEASUREMENTS $\ldots \ldots \ldots \ldots \ldots \ldots \ldots \ldots \ldots . \ldots . \ldots . \ldots$

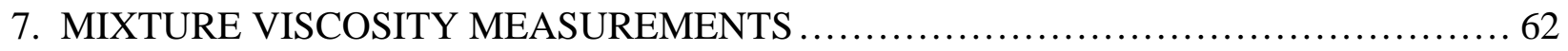

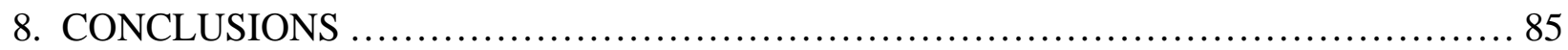

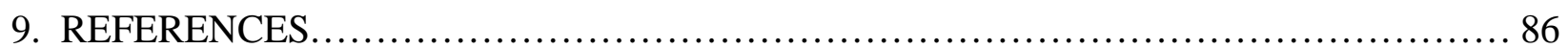

APPENDIX A-MANUSCRIPT “An Equation of State for Pentafluoroethane (HFC-125) for Temperatures from the Triple Point (172.52 K) to $500 \mathrm{~K}$ and Pressures to $60 \mathrm{MPa}$ ". Attachment A

APPENDIX B-MANUSCRIPT "Equations of State for Mixtures of R-32, R-125, R-134a, R-143a, and R152a"... Attachment B 


\section{LIST OF TABLES}

1. Isochoric heat capacity data measured for R125 ..............................

2. Coefficients and exponents to the R125 equation of state....................... 12

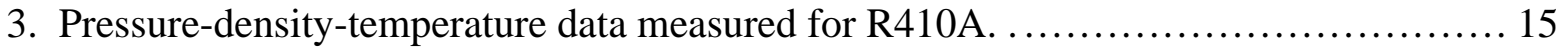

4. Isochoric heat capacity and P- $\rho$-T data measured for R410A. .................... 19

5. Pure fluid equations of state for refrigerants used in the mixture model. ............. 25

6. Coefficients and exponents of the mixture equations. ........................... 25

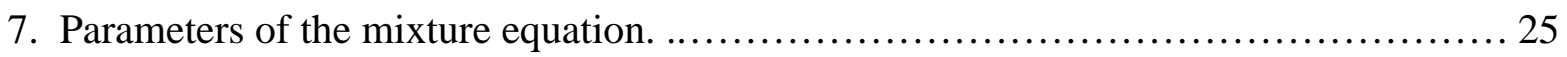

8. Thermal conductivity for R410A measured by the steady-state technique............. 33

9. Thermal conductivity for R410A measured by the transient hot-wire technique. ...... 35

10. Thermal conductivity for R507A measured by the steady-state technique. ........... 47

11. Thermal conductivity for R507A measured by the transient hot-wire technique. ...... 49

12. Experimental viscosities of the mixture R507A (0.5 R143a + 0.5 R125 by mass) as measured in the torsional crystal viscometer. .................................. 75

13. Experimental viscosities of the mixture R410A $(0.5 \mathrm{R} 32+0.5 \mathrm{R} 125$ by mass $)$ as measured in the torsional crystal viscometer. ................................. 79

14. Saturated-liquid viscosities for the mixture R410A ( $0.5 \mathrm{R} 32+0.5 \mathrm{R} 125$ by mass) measured in the sealed gravitational capillary viscometer. 


\section{LIST OF FIGURES}

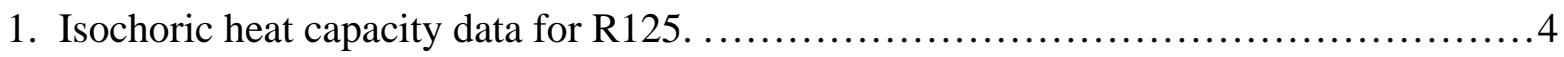

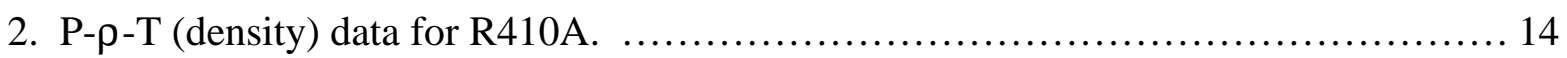

3. Isochoric heat capacity data for R410A. ....................................... 18

4. Location of measured thermal conductivity data points for R410A. ................ 27

5. Location of measured thermal conductivity data points for R507A. ............... 28

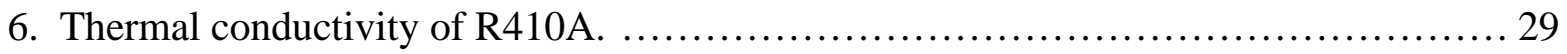

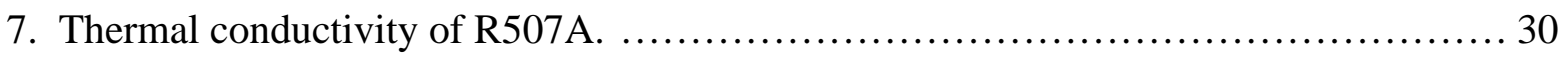

8. Deviations between the measured thermal conductivities for R410A and the model in REFPROP. .................................................. 31

9. Deviations between the measured thermal conductivities for R507A and the model in REFPROP.

10. Conductances $G$ at $39 \mathrm{kHz}$ of the mixture R507A as measured in the torsional crystal viscometer.

11. Density dependence of experimental viscosities of the mixture R507A as measured in the torsional crystal viscometer.

12. Percent deviations between experimental viscosities of the mixture R507A as measured in the torsional crystal viscometer and data calculated with the model in REFPROP.

13. Percent deviations between literature data for the viscosity of the binary system $\mathrm{R} 143 \mathrm{a}+\mathrm{R} 125$ and data calculated with the model in REFPROP. 70

14. Conductances $G$ at $39 \mathrm{kHz}$ of the mixture R410A as measured in the torsional crystal viscometer.

15. Density dependence of experimental viscosities of the mixture R410A as measured in the torsional crystal and capillary viscometers.

16. Percent deviations between experimental viscosities of the mixture R410A as measured in the torsional crystal viscometer and in the sealed gravitational capillary viscometer and data calculated with the model in REFPROP.

17. Percent deviations between literature data for the viscosity of the binary system $\mathrm{R} 32+\mathrm{R} 125$ and data calculated with the model in REFPROP. 


\section{NOMENCLATURE}

$\begin{array}{ll}a & \text { Helmholtz energy }[\mathrm{J} / \mathrm{mol}] \\ C_{V} & \text { heat capacity at constant volume }[\mathrm{kJ} /(\mathrm{kg}-\mathrm{K})] \\ C_{p} & \text { heat capacity at constant pressure }[\mathrm{kJ} /(\mathrm{kg}-\mathrm{K})] \\ d, l, t & \text { exponents in mixture model } \\ f^{*} & \text { resonant frequency }[\mathrm{Hz}] \\ F_{i j} & \text { generalized mixture parameter } \\ G & \text { conductance }[\mu \mathrm{S}] \\ i, j, l, m & \text { exponents in equation of state } \\ i, k & \text { index for summation } \\ n & \text { number of components in mixture } \\ N & \text { numerical coefficient } \\ p, P & \text { pressure }[\mathrm{MPa}] \\ R & \text { gas constant }[8.314472 \mathrm{~J} /(\text { mol-K) }] \\ s & \text { standard deviation in a measured quantity } \\ T & \text { temperature }[\mathrm{K}] \\ u & \text { parameter in heat capacity }(\text { Equation } 4) \\ x & \text { mixture composition }[\text { mole fraction] } \\ \alpha & \text { dimensionless Helmholtz energy }(\alpha=a / R T) \\ \beta & \text { exponent in Equation } 6 \\ \delta & \text { reduced density } \\ \Delta & \text { difference between calculated and experimental quantity } \\ \Delta \mathrm{f} & \text { bandwidth of resonance }[\mathrm{Hz}] \\ \eta & \text { viscosity [mPa-s] } \\ \lambda & \text { thermal conductivity }[\mathrm{W} / \mathrm{m} \cdot \mathrm{K}] \\ \rho & \text { density }\left[\mathrm{kg} / \mathrm{m}^{3}(\text { data); mol/L }(\text { equation of state })]\right. \\ \tau & \text { inverse reduced temperature }\left(\tau=\mathrm{T}_{c} / \mathrm{T}\right) \\ \theta & \text { dimensionless temperature }\left(\theta=1-\mathrm{T} / \mathrm{T}_{\mathrm{c}}\right) \\ \xi & \text { density parameter in mixture model }[\mathrm{L} / \mathrm{mol}] \\ \zeta & \text { temperature parameter in mixture model }[\mathrm{K}]\end{array}$

Note: Symbols used only once are defined where they occur. 


$\begin{array}{ll}\text { Subscripts } & \\ \text { c } & \text { critical point } \\ \text { calc } & \text { calculated value } \\ \text { exp } & \text { experimental value } \\ \text { liq } & \text { liquid } \\ \text { lit } & \text { value from literature } \\ \text { red } & \text { reduced } \\ \text { REFPROP } & \text { value calculated with the NIST REFPROP database } \\ \text { vap } & \text { vapor } \\ \sigma & \text { saturation } \\ 0 & \text { reference state (vacuum for viscosity measurements) } \\ & \\ \text { Superscripts } & \\ \text { E } & \text { excess (departure from ideal mixing) } \\ \text { idmix } & \text { ideal mixing } \\ \text { r } & \text { residual or real-gas contribution } \\ 0 & \text { ideal gas state }\end{array}$




\section{INTRODUCTION}

The Working Fluids Subcommittee of the ARTI 21-CR program has identified the performance of R410A at high ambient temperatures to be a very high research priority. This project has addressed the highest priority data data needs. A parallel effort by the NIST Thermal Machinery Group under the direction of Dr. Piotr Domanski has investigated the cycle performance of blends operating near the refrigerant critical point and the modeling of cycles operating at such conditions. Further analysis of the performance was carried out by Dr. Keith Rice at Oak Ridge National Laboratory. Their work is detailed in companion reports. The work at NIST was jointly funded by the Air-Conditioning and Refrigeration Technology Institute (ARTI) under their 21-CR program, the Building Equipment Division of the U.S. Department of Energy, and the National Institute of Standards and Technology (NIST).

The 50/50 (by mass) blend of R32 and R125 is known as R410A. It is the replacement of choice for R22 in many heat pumps and air-conditioners. R404A is the blend of R125, R143a, and $\mathrm{R} 134 \mathrm{a}$ at a composition of 44/52/4 mass percent; it is the replacement for R502 in many applications. Compared to R22, R410A and R404A operate at higher pressures and provide a greater refrigeration capacity. Both of these features stem from the lower critical temperatures of the blends. The critical temperatures of R410A $\left(343 \mathrm{~K}\right.$ or $\left.70{ }^{\circ} \mathrm{C}\right)$ and R404A $\left(345 \mathrm{~K}\right.$ or $\left.\left.72{ }^{\circ} \mathrm{C}\right)\right]$ are significantly lower than the R22 critical temperature of $369 \mathrm{~K}$ or $96{ }^{\circ} \mathrm{C}$. Or, stated differently, for a given ambient temperature, R410A and R404A operate closer to their respective critical temperatures than $\mathrm{R} 22$. At high ambient temperatures, the condensing temperature is near the critical temperature, and a significant energy penalty has been measured.

To understand the causes of the energy penalty at high ambient conditions, detailed modeling of the cycle is a valuable tool, and this requires reliable property data. But, there are very few data for the blends near their critical temperatures, and the limited data at lower temperatures indicates that present mixture models are not adequate.

To address the observed deficiencies in the existing mixture model, we have carried out measurements on R125 to fill in large gaps in the available data in the critical and supercritical regions (Task 1a). These data were used to develop an improved and comprehensive equation of state (Task 1b). Similar measurements on the blends (Task 1c), combined with the new R125 equation of state, were used to develop an improved mixture model for blends of R32, R125, R143a, R134a, and R152a (Task 1d). The results of this work have been incorporated into version 7 of the NIST REFPROP database [Lemmon et al. 2002], which will be released in the summer of 2002.

The focus of the properties work was on the thermodynamic properties and equation-of-state modeling, but we also addressed the transport properties of viscosity and thermal conductivity. Working from recent literature surveys (Yata, 1998 and Tanaka and Sotani, 1998) we identified key gaps in the available transport properties for the blends of interest and have measured those properties and compared them to the current model in REFPROP (Task 1e). The modeling of mixture transport properties was not part of the current project.

Fluid property data are important from at least three aspects. First, data impact the energy efficiency. A workable solution to replacing R22 could have been arrived at with only minimal data. But finding the fluid which will yield the best performance among many possibilities requires accurate data to distinguish small differences between fluids. Second, the design of refrigeration equipment increasingly involves the use of detailed simulation models. The results from such models can be no better than the reliability of the input data, of which, the fluid properties are among the most important. Third, the rating of compressors and other components requires property data to convert the raw temperature, pressure, and flow measurements from a 
compressor calorimeter test into the efficiencies quoted on a product specification sheet. For this application, consensus is more important than absolute accuracy.

The project was composed of the following tasks. The results of each task are detailed in subsequent sections of this report.

\section{Task 1a-R125 Heat Capacity Measurements}

Measure the isochoric heat capacity $(\mathrm{Cv})$ of R125 over the temperature range of $300-400 \mathrm{~K}$ with pressures extending from near saturation to $20 \mathrm{MPa}$. These measurements will be carried out along isochores (lines of constant density) with continuous scanning of the energy input and temperature rise.

\section{Task 1b-R125 Equation of State Development}

Collect R125 literature data for vapor pressure, saturated liquid density, P- $\rho-\mathrm{T}$ behavior, heat capacity, and speed of sound. Using the literature data together with the data measured in Task 1a, develop a comprehensive, high-accuracy equation of state for the thermodynamic properties of R125. The temperature range will extend from the triple point temperature of $172.52 \mathrm{~K}$ to at least $450 \mathrm{~K}$, with pressures to $40 \mathrm{MPa}$. Incorporate this equation into the NIST REFPROP database.

\section{Task 1c-Mixture P- $\rho$-T and Heat Capacity Measurements}

Measure the pressure-density-temperature (P- $\rho-\mathrm{T}$ ) behavior of R410A [R32/125 (50/50)] over the temperature range of $200-400 \mathrm{~K}$ with pressures extending from near saturation to $35 \mathrm{MPa}$. These measurements will be carried out along isochores with $5-10$ data points per isochore, depending on the density.

Measure the isochoric heat capacity $\left(\mathrm{C}_{\mathrm{V}}\right)$ of R410A [R32/125 (50/50)] over the temperature range of $300-400 \mathrm{~K}$ with pressures extending from near saturation to $20 \mathrm{MPa}$. These measurements will be carried out along isochores with continuous scanning of the energy input and temperature rise.

\section{Task 1d-Mixture Modeling}

Collect literature data for dew and bubble point pressures, saturated liquid density, P- $\rho-\mathrm{T}$ behavior, heat capacity, and speed of sound for HFC mixtures, including R32/125, R125/143a, $\mathrm{R} 125 / 134 \mathrm{a}, \mathrm{R} 143 \mathrm{a} / 134 \mathrm{a}, \mathrm{R} 32 / 134 \mathrm{a}$, and R134a/152a. Using the literature data together with the data measured in Task 1c, develop a comprehensive, high-accuracy mixture model for the thermodynamic properties of HFC blends. The temperature range will extend from $200 \mathrm{~K}$ to at least $400 \mathrm{~K}$, with pressures to $35 \mathrm{MPa}$. Incorporate this model into the NIST REFPROP database.

\section{Task 1e-Mixture Transport Property Measurements}

For the R410A mixture [R32/125 (50/50)] and for the R507A mixuture [R125/143a (50/50)] measure the viscosity and thermal conductivity along isotherms covering the temperature range $300-400 \mathrm{~K}$ with pressures to $30 \mathrm{MPa}$. Approximately 20 data points per isotherm will be measured. Compare these data to the present mixture model in the NIST REFPROP database. 


\section{R125 HEAT CAPACITY MEASUREMENTS}

Background. At the outset of the project, we suspected that deficiencies in the pure fluid R125 equation of state may have been at least partly responsible for the deviations seen with the R32/125 mixtures. (Mixture properties can be no better than the constituent pure fluids.) All of the R125-containing mixtures for which we have heat capacity data show higher deviations with our mixture model than those which do not contain R125. There was no reason to suspect that R125 would present any particular difficulties in this regards.

There was an enormous gap in the existing pure-fluid data for R125 in the vicinity of the critical point for any sort of thermal properties (i.e. $\mathrm{C}_{\mathrm{v}}, \mathrm{C}_{\mathrm{p}}$, enthalpy, or speed of sound). There are three or four individual data points for $\mathrm{C}_{\mathrm{V}}$ above the $\mathrm{R} 125$ critical temperature of $339 \mathrm{~K}$, but they are at much higher pressures (about $20 \mathrm{MPa}$ versus the critical pressure of $3.6 \mathrm{MPa}$ ). There are a few liquid-phase speed of sound measurements, but those are at sub-critical temperatures and pressures at least double the critical pressure. There are vapor-phase speed of sound data that extend to $400 \mathrm{~K}$ (i.e. $61 \mathrm{~K}$ above the critical temperature), but the maximum pressure for these is $1 \mathrm{MPa}$-much lower than the critical pressure. It is our experience that heat capacity data is absolutely required to develop an adequate equation of state. This is especially so in the critical region.

For these reasons, the project included measurements of the heat capacity which extended well above critical and overlaped with existing data. These data were applied to the development of the R125 equation of state described in the next section. The large deviations seen with the old mixture model for the R32/125 mixtures were found to be largely a result of the peculiar behavior of R32, as detailed in Section 5, but nonetheless, a much-improved equation of state has resulted for this important fluid.

Experimental. The R125 used in this work was "electronic grade" material supplied by the Dupont Company ${ }^{1}$. The analysis provided by the manufacturer stated a purity of $99.997 \%$ with a water content less than $5 \mathrm{ppm}$. Our analysis by gas chromatography confirmed that it was of very high purity apart from a small $(0.02 \%)$ amout of air in the vapor phase. The air and any other light inorganics which may have been present were removed by repeatedly freezing the sample in liquid nitrogen, evacuating the vapor space, and thawing.

Measurements of the heat capacity of R125 were carried out using a constant-volume adiabatic calorimeter. The calorimeter has twin cells of constant volume that are operated in a differential manner. The heat required to raise the temperature of the cell containing the sample is measured relative to that required for a matched cell that is evacuated. Such an approach cancels some of the parasitic heat flows which can compromise the accuracy of the measurement. The supply gas was condensed (single phase) into the measurement cell at temperatures near $220 \mathrm{~K}$ and subsequently pressurized by warming. At the end of each isochore, a portion of the sample was carefully condensed into an external weighing cylinder that was cooled with liquid nitrogen. The weight change measured between each isochore and the final weight of the sample remaining in the cell after the last isochore provide the mass of the sample in the cell for each isochore. The high-temperature isochoric calorimeter was used to measure the isochoric specific heat $\left(\mathrm{C}_{\mathrm{v}}\right)$ of pure R125. The apparatus and procedures are detailed by Magee et al. (1998).

The measurements cover the temperature region from 305 to $397 \mathrm{~K}$ along 7 isochores (lines of constant density) from 584 to $1202 \mathrm{~kg} / \mathrm{m}^{3}$ with pressures up to $20 \mathrm{MPa}$. Repetitions of several of

\footnotetext{
${ }^{1}$ Suppliers are identified to fully document the details of the work. Mention of any supplier does not imply endorsement by NIST nor does it imply that the material is necessarily the best suited for the purpose.
} 
the isochores were also carried out. The loci of the measured points on pressure-temperature coordinates are shown in Figure 1. The data themselves are given in Table 1. A comparison of these data to the R125 equation of state is given in the manuscript describing the equation (Appendix A of this report).

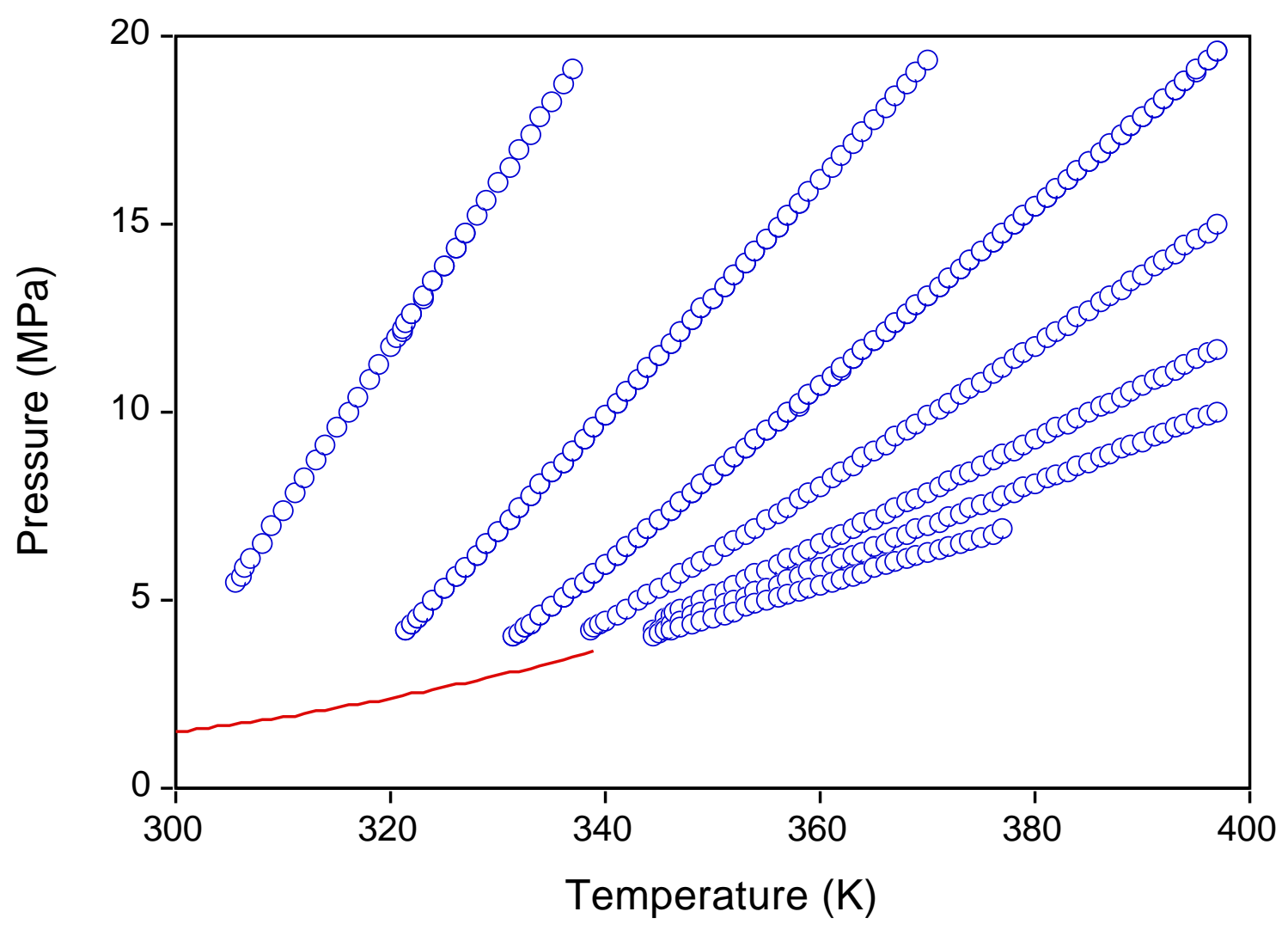

Figure 1. Isochoric heat capacity data for R125. The symbols indicate measured $\mathrm{C}_{\mathrm{V}}$ data points and the line indicates the vapor pressure. 
Table 1. Isochoric heat capacity data measured for R125.

\begin{tabular}{|c|c|c|c|c|c|}
\hline $\mathrm{T}(\mathrm{K})$ & $\mathrm{P}(\mathrm{MPa})$ & $\mathrm{C}_{\mathrm{V}}(\mathrm{kJ} / \mathrm{kg}-\mathrm{K})$ & $\mathrm{T}(\mathrm{K})$ & $\mathrm{P}(\mathrm{MPa})$ & $\mathrm{C}_{\mathrm{V}}(\mathrm{kJ} / \mathrm{kg}-\mathrm{K})$ \\
\hline 305.5 & 5.418 & 0.8280 & 326.0 & 5.571 & 0.8668 \\
\hline 306.0 & 5.635 & 0.8410 & 327.0 & 5.878 & 0.8700 \\
\hline 306.5 & 5.851 & 0.8378 & 328.0 & 6.181 & 0.8741 \\
\hline 307.0 & 6.067 & 0.8351 & 329.0 & 6.486 & 0.8704 \\
\hline 308.0 & 6.498 & 0.8318 & 330.0 & 6.798 & 0.8735 \\
\hline 309.0 & 6.933 & 0.8312 & 331.0 & 7.108 & 0.8747 \\
\hline 310.0 & 7.365 & 0.8293 & 332.0 & 7.413 & 0.8739 \\
\hline 311.0 & 7.797 & 0.8317 & 333.0 & 7.721 & 0.8766 \\
\hline 312.0 & 8.230 & 0.8371 & 334.0 & 8.034 & 0.8740 \\
\hline 313.0 & 8.665 & 0.8331 & 335.0 & 8.344 & 0.8737 \\
\hline 314.0 & 9.098 & 0.8375 & 336.0 & 8.650 & 0.8697 \\
\hline 315.0 & 9.531 & 0.8355 & 337.0 & 8.962 & 0.8720 \\
\hline 316.0 & 9.964 & 0.8343 & 338.0 & 9.275 & 0.8723 \\
\hline 317.0 & 10.399 & 0.8356 & 339.0 & 9.586 & 0.8736 \\
\hline 318.0 & 10.833 & 0.8345 & 340.0 & 9.895 & 0.8779 \\
\hline 319.0 & 11.267 & 0.8367 & 341.0 & 10.207 & 0.8759 \\
\hline 320.0 & 11.700 & 0.8365 & 342.0 & 10.521 & 0.8747 \\
\hline 321.0 & 12.135 & 0.8380 & 343.0 & 10.833 & 0.8732 \\
\hline 322.0 & 12.569 & 0.8365 & 344.0 & 11.145 & 0.8773 \\
\hline 323.0 & 13.004 & 0.8389 & 345.0 & 11.457 & 0.8765 \\
\hline 324.0 & 13.436 & 0.8380 & 346.0 & 11.769 & 0.8754 \\
\hline 325.0 & 13.871 & 0.8388 & 347.0 & 12.083 & 0.8773 \\
\hline 326.0 & 14.305 & 0.8425 & 348.0 & 12.395 & 0.8763 \\
\hline 327.0 & 14.740 & 0.8399 & 349.0 & 12.708 & 0.8788 \\
\hline 320.5 & 11.939 & 0.8592 & 350.0 & 13.020 & 0.8821 \\
\hline 321.0 & 12.156 & 0.8514 & 351.0 & 13.335 & 0.8828 \\
\hline 321.5 & 12.372 & 0.8536 & 352.0 & 13.649 & 0.8825 \\
\hline 322.0 & 12.592 & 0.8540 & 353.0 & 13.963 & 0.8804 \\
\hline 323.0 & 13.027 & 0.8539 & 354.0 & 14.277 & 0.8832 \\
\hline 324.0 & 13.461 & 0.8544 & 355.0 & 14.592 & 0.8823 \\
\hline 325.0 & 13.895 & 0.8559 & 356.0 & 14.904 & 0.8822 \\
\hline 326.0 & 14.332 & 0.8559 & 357.0 & 15.220 & 0.8842 \\
\hline 327.0 & 14.766 & 0.8563 & 358.0 & 15.536 & 0.8853 \\
\hline 328.0 & 15.201 & 0.8610 & & & \\
\hline 329.0 & 15.634 & 0.8572 & 321.5 & 4.197 & 0.8537 \\
\hline 330.0 & 16.069 & 0.8629 & 322.0 & 4.347 & 0.8614 \\
\hline 331.0 & 16.507 & 0.8641 & 322.5 & 4.501 & 0.8685 \\
\hline 332.0 & 16.942 & 0.8650 & 323.0 & 4.655 & 0.8676 \\
\hline 333.0 & 17.373 & 0.8649 & 324.0 & 4.957 & 0.8668 \\
\hline 334.0 & 17.807 & 0.8597 & 325.0 & 5.261 & 0.8697 \\
\hline 335.0 & 18.245 & 0.8627 & 326.0 & 5.570 & 0.8679 \\
\hline 336.0 & 18.680 & 0.8632 & 327.0 & 5.875 & 0.8660 \\
\hline \multirow[t]{2}{*}{337.0} & 19.114 & 0.8691 & 328.0 & 6.181 & 0.8709 \\
\hline & & & 329.0 & 6.488 & 0.8667 \\
\hline 321.5 & 4.198 & 0.8845 & 330.0 & 6.799 & 0.8712 \\
\hline 322.0 & 4.349 & 0.8795 & 331.0 & 7.104 & 0.8719 \\
\hline 322.5 & 4.503 & 0.8817 & 332.0 & 7.414 & 0.8704 \\
\hline 323.0 & 4.656 & 0.8775 & 333.0 & 7.720 & 0.8680 \\
\hline 324.0 & 4.959 & 0.8686 & 334.0 & 8.032 & 0.8633 \\
\hline 325.0 & 5.261 & 0.8691 & 335.0 & 8.344 & 0.8720 \\
\hline
\end{tabular}


Table 1. $\mathrm{C}_{\mathrm{V}}$ for R125 (continued)

\begin{tabular}{|c|c|c|c|c|c|}
\hline $\mathrm{T}(\mathrm{K})$ & $\mathrm{P}(\mathrm{MPa})$ & $\mathrm{C}_{\mathrm{V}}(\mathrm{kJ} / \mathrm{kg}-\mathrm{K})$ & $\mathrm{T}(\mathrm{K})$ & $\mathrm{P}(\mathrm{MPa})$ & $\mathrm{C}_{\mathrm{V}}(\mathrm{kJ} / \mathrm{kg}-\mathrm{K})$ \\
\hline 336.0 & 8.651 & 0.8698 & 344.0 & 6.869 & 0.9185 \\
\hline 337.0 & 8.962 & 0.8735 & 345.0 & 7.101 & 0.8922 \\
\hline 338.0 & 9.273 & 0.8747 & 346.0 & 7.334 & 0.8885 \\
\hline 339.0 & 9.586 & 0.8692 & 347.0 & 7.570 & 0.8857 \\
\hline 340.0 & 9.895 & 0.8737 & 348.0 & 7.804 & 0.8852 \\
\hline 341.0 & 10.208 & 0.8751 & 349.0 & 8.037 & 0.8882 \\
\hline 342.0 & 10.520 & 0.8723 & 350.0 & 8.273 & 0.8888 \\
\hline 343.0 & 10.832 & 0.8740 & 351.0 & 8.509 & 0.8848 \\
\hline 344.0 & 11.145 & 0.8747 & 352.0 & 8.745 & 0.8918 \\
\hline 345.0 & 11.455 & 0.8724 & 353.0 & 8.981 & 0.8908 \\
\hline 346.0 & 11.769 & 0.8758 & 354.0 & 9.216 & 0.8904 \\
\hline 347.0 & 12.081 & 0.8776 & 355.0 & 9.454 & 0.8937 \\
\hline 348.0 & 12.396 & 0.8786 & 356.0 & 9.691 & 0.8894 \\
\hline 349.0 & 12.708 & 0.8840 & 357.0 & 9.929 & 0.8936 \\
\hline 350.0 & 13.022 & 0.8808 & 358.0 & 10.164 & 0.8907 \\
\hline 351.0 & 13.334 & 0.8822 & 359.0 & 10.405 & 0.8917 \\
\hline 352.0 & 13.651 & 0.8851 & 360.0 & 10.642 & 0.8944 \\
\hline 353.0 & 13.964 & 0.8817 & 361.0 & 10.880 & 0.8886 \\
\hline 354.0 & 14.276 & 0.8831 & 362.0 & 11.116 & 0.8927 \\
\hline 355.0 & 14.590 & 0.8832 & 363.0 & 11.358 & 0.8869 \\
\hline 356.0 & 14.907 & 0.8847 & 364.0 & 11.598 & 0.8939 \\
\hline 357.0 & 15.221 & 0.8854 & 365.0 & 11.834 & 0.8935 \\
\hline 358.0 & 15.533 & 0.8899 & 366.0 & 12.072 & 0.8943 \\
\hline 359.0 & 15.847 & 0.8883 & 367.0 & 12.313 & 0.9003 \\
\hline 360.0 & 16.163 & 0.8855 & 368.0 & 12.554 & 0.8930 \\
\hline 361.0 & 16.478 & 0.8907 & 369.0 & 12.793 & 0.9015 \\
\hline 362.0 & 16.792 & 0.8874 & 370.0 & 13.031 & 0.8971 \\
\hline 363.0 & 17.104 & 0.8862 & 371.0 & 13.271 & 0.8978 \\
\hline 364.0 & 17.421 & 0.8880 & 372.0 & 13.513 & 0.9032 \\
\hline 365.0 & 17.736 & 0.8860 & 373.0 & 13.754 & 0.9008 \\
\hline 366.0 & 18.049 & 0.8874 & 374.0 & 13.992 & 0.9051 \\
\hline 367.0 & 18.361 & 0.8880 & 375.0 & 14.233 & 0.9060 \\
\hline 368.0 & 18.679 & 0.8898 & 376.0 & 14.475 & 0.9008 \\
\hline 369.0 & 18.994 & 0.8920 & 377.0 & 14.713 & 0.9008 \\
\hline \multirow[t]{2}{*}{370.0} & 19.305 & 0.8885 & 378.0 & 14.955 & 0.9013 \\
\hline & & & 379.0 & 15.197 & 0.9233 \\
\hline 331.5 & 4.003 & 0.9279 & 380.0 & 15.437 & 0.9241 \\
\hline 332.0 & 4.114 & 0.8952 & 381.0 & 15.675 & 0.9242 \\
\hline 332.5 & 4.228 & 0.8912 & 382.0 & 15.916 & 0.9275 \\
\hline 333.0 & 4.341 & 0.8898 & 383.0 & 16.160 & 0.9094 \\
\hline 334.0 & 4.567 & 0.8801 & 384.0 & 16.401 & 0.9192 \\
\hline 335.0 & 4.794 & 0.8911 & 385.0 & 16.640 & 0.9196 \\
\hline 336.0 & 5.023 & 0.8917 & 386.0 & 16.879 & 0.9197 \\
\hline 337.0 & 5.249 & 0.8858 & 387.0 & 17.122 & 0.9175 \\
\hline 338.0 & 5.479 & 0.8896 & 388.0 & 17.366 & 0.9279 \\
\hline 339.0 & 5.711 & 0.8890 & 389.0 & 17.607 & 0.9353 \\
\hline 340.0 & 5.941 & 0.9061 & 390.0 & 17.846 & 0.9295 \\
\hline 341.0 & 6.170 & 0.9359 & 391.0 & 18.086 & 0.9328 \\
\hline 342.0 & 6.403 & 0.9352 & 392.0 & 18.330 & 0.9290 \\
\hline 343.0 & 6.637 & 0.9391 & 393.0 & 18.572 & 0.9291 \\
\hline
\end{tabular}


Table 1. $\mathrm{C}_{\mathrm{V}}$ for R125 (continued)

\begin{tabular}{|c|c|c|c|c|c|}
\hline $\mathrm{T}(\mathrm{K})$ & $\mathrm{P}(\mathrm{MPa})$ & $\mathrm{C}_{\mathrm{V}}(\mathrm{kJ} / \mathrm{kg}-\mathrm{K})$ & $\mathrm{T}(\mathrm{K})$ & $\mathrm{P}(\mathrm{MPa})$ & $\mathrm{C}_{\mathrm{V}}(\mathrm{kJ} / \mathrm{kg}-\mathrm{K})$ \\
\hline 394.0 & 18.812 & 0.9452 & 375.0 & 14.235 & 0.9151 \\
\hline 395.0 & 19.050 & 0.9418 & 376.0 & 14.475 & 0.9164 \\
\hline 396.0 & 19.294 & 0.9452 & 377.0 & 14.716 & 0.9116 \\
\hline \multirow[t]{2}{*}{397.0} & 19.536 & 0.9578 & 378.0 & 14.956 & 0.9145 \\
\hline & & & 379.0 & 15.197 & 0.9132 \\
\hline 331.5 & 4.002 & 0.9288 & 380.0 & 15.439 & 0.9158 \\
\hline 332.0 & 4.117 & 0.9134 & 381.0 & 15.679 & 0.9190 \\
\hline 332.5 & 4.229 & 0.9060 & 382.0 & 15.919 & 0.9183 \\
\hline 333.0 & 4.342 & 0.8985 & 383.0 & 16.160 & 0.9181 \\
\hline 334.0 & 4.568 & 0.8932 & 384.0 & 16.402 & 0.9223 \\
\hline 335.0 & 4.797 & 0.8940 & 385.0 & 16.643 & 0.9217 \\
\hline 336.0 & 5.024 & 0.8924 & 386.0 & 16.883 & 0.9245 \\
\hline 337.0 & 5.251 & 0.8934 & 387.0 & 17.125 & 0.9285 \\
\hline 338.0 & 5.482 & 0.8933 & 388.0 & 17.366 & 0.9237 \\
\hline 339.0 & 5.713 & 0.8903 & 389.0 & 17.608 & 0.9269 \\
\hline 340.0 & 5.942 & 0.8909 & 390.0 & 17.848 & 0.9268 \\
\hline 341.0 & 6.172 & 0.8902 & 391.0 & 18.091 & 0.9270 \\
\hline 342.0 & 6.405 & 0.8864 & 392.0 & 18.332 & 0.9320 \\
\hline 343.0 & 6.637 & 0.8871 & 393.0 & 18.573 & 0.9294 \\
\hline 344.0 & 6.870 & 0.8900 & 394.0 & 18.814 & 0.9301 \\
\hline 345.0 & 7.101 & 0.8925 & 395.0 & 19.055 & 0.9323 \\
\hline 346.0 & 7.336 & 0.8925 & 396.0 & 19.297 & 0.9322 \\
\hline 347.0 & 7.570 & 0.8990 & 397.0 & 19.538 & 0.9343 \\
\hline 348.0 & 7.805 & 0.8952 & & & \\
\hline 349.0 & 8.039 & 0.8981 & 338.5 & 4.150 & 0.9487 \\
\hline 350.0 & 8.274 & 0.8997 & 339.0 & 4.237 & 0.9414 \\
\hline 351.0 & 8.512 & 0.8972 & 339.5 & 4.324 & 0.9414 \\
\hline 352.0 & 8.746 & 0.8980 & 340.0 & 4.412 & 0.9314 \\
\hline 353.0 & 8.983 & 0.8977 & 341.0 & 4.584 & 0.9189 \\
\hline 354.0 & 9.218 & 0.8931 & 342.0 & 4.760 & 0.9202 \\
\hline 355.0 & 9.456 & 0.8963 & 343.0 & 4.936 & 0.9128 \\
\hline 356.0 & 9.693 & 0.8999 & 344.0 & 5.113 & 0.9192 \\
\hline 357.0 & 9.931 & 0.9001 & 345.0 & 5.288 & 0.9221 \\
\hline 358.0 & 10.168 & 0.9045 & 346.0 & 5.466 & 0.9123 \\
\hline 359.0 & 10.404 & 0.8992 & 347.0 & 5.644 & 0.9126 \\
\hline 360.0 & 10.644 & 0.8989 & 348.0 & 5.825 & 0.9155 \\
\hline 361.0 & 10.882 & 0.9005 & 349.0 & 6.005 & 0.9136 \\
\hline 362.0 & 11.120 & 0.9005 & 350.0 & 6.183 & 0.9111 \\
\hline 363.0 & 11.357 & 0.9032 & 351.0 & 6.363 & 0.9195 \\
\hline 364.0 & 11.597 & 0.9070 & 352.0 & 6.546 & 0.9175 \\
\hline 365.0 & 11.836 & 0.9065 & 353.0 & 6.728 & 0.9178 \\
\hline 366.0 & 12.075 & 0.9090 & 354.0 & 6.909 & 0.9248 \\
\hline 367.0 & 12.316 & 0.9131 & 355.0 & 7.093 & 0.9201 \\
\hline 368.0 & 12.555 & 0.9115 & 356.0 & 7.276 & 0.9155 \\
\hline 369.0 & 12.794 & 0.9137 & 357.0 & 7.458 & 0.9123 \\
\hline 370.0 & 13.033 & 0.9080 & 358.0 & 7.640 & 0.9079 \\
\hline 371.0 & 13.274 & 0.9082 & 359.0 & 7.825 & 0.9103 \\
\hline 372.0 & 13.514 & 0.9093 & 360.0 & 8.010 & 0.9118 \\
\hline 373.0 & 13.754 & 0.9127 & 361.0 & 8.193 & 0.9228 \\
\hline 374.0 & 13.993 & 0.9161 & 362.0 & 8.377 & 0.9253 \\
\hline
\end{tabular}


Table 1. $\mathrm{C}_{\mathrm{V}}$ for R125 (continued)

\begin{tabular}{|c|c|c|c|c|c|}
\hline $\mathrm{T}(\mathrm{K})$ & $\mathrm{P}(\mathrm{MPa})$ & $\mathrm{C}_{\mathrm{V}}(\mathrm{kJ} / \mathrm{kg}-\mathrm{K})$ & $\mathrm{T}(\mathrm{K})$ & $\mathrm{P}(\mathrm{MPa})$ & $\mathrm{C}_{\mathrm{V}}(\mathrm{kJ} / \mathrm{kg}-\mathrm{K})$ \\
\hline 363.0 & 8.563 & 0.9226 & 358.0 & 6.187 & 0.9504 \\
\hline 364.0 & 8.750 & 0.9228 & 359.0 & 6.325 & 0.9360 \\
\hline 365.0 & 8.936 & 0.9161 & 360.0 & 6.463 & 0.9363 \\
\hline 366.0 & 9.121 & 0.9156 & 361.0 & 6.598 & 0.9290 \\
\hline 367.0 & 9.307 & 0.9183 & 362.0 & 6.736 & 0.9190 \\
\hline 368.0 & 9.494 & 0.9164 & 363.0 & 6.875 & 0.9545 \\
\hline 369.0 & 9.679 & 0.9196 & 364.0 & 7.013 & 0.9502 \\
\hline 370.0 & 9.864 & 0.9193 & 365.0 & 7.149 & 0.9547 \\
\hline 371.0 & 10.052 & 0.9216 & 366.0 & 7.288 & 0.9626 \\
\hline 372.0 & 10.239 & 0.9255 & 367.0 & 7.427 & 0.9255 \\
\hline 373.0 & 10.425 & 0.9264 & 368.0 & 7.568 & 0.9335 \\
\hline 374.0 & 10.612 & 0.9266 & 369.0 & 7.705 & 0.9349 \\
\hline 375.0 & 10.799 & 0.9243 & 370.0 & 7.846 & 0.9328 \\
\hline 376.0 & 10.988 & 0.9234 & 371.0 & 7.984 & 0.9324 \\
\hline 377.0 & 11.176 & 0.9264 & 372.0 & 8.126 & 0.9282 \\
\hline 378.0 & 11.364 & 0.9296 & 373.0 & 8.265 & 0.9333 \\
\hline 379.0 & 11.552 & 0.9341 & 374.0 & 8.407 & 0.9385 \\
\hline 380.0 & 11.742 & 0.9384 & 375.0 & 8.544 & 0.9342 \\
\hline 381.0 & 11.930 & 0.9374 & 376.0 & 8.686 & 0.9378 \\
\hline 382.0 & 12.118 & 0.9416 & 377.0 & 8.828 & 0.9436 \\
\hline 383.0 & 12.308 & 0.9348 & 378.0 & 8.967 & 0.9329 \\
\hline 384.0 & 12.497 & 0.9404 & 379.0 & 9.107 & 0.9468 \\
\hline 385.0 & 12.685 & 0.9385 & 380.0 & 9.251 & 0.9471 \\
\hline 386.0 & 12.874 & 0.9348 & 381.0 & 9.393 & 0.9402 \\
\hline 387.0 & 13.062 & 0.9394 & 382.0 & 9.532 & 0.9446 \\
\hline 388.0 & 13.253 & 0.9380 & 383.0 & 9.675 & 0.9399 \\
\hline 389.0 & 13.440 & 0.9388 & 384.0 & 9.816 & 0.9367 \\
\hline 390.0 & 13.630 & 0.9401 & 385.0 & 9.956 & 0.9354 \\
\hline 391.0 & 13.819 & 0.9436 & 386.0 & 10.099 & 0.9445 \\
\hline 392.0 & 14.010 & 0.9383 & 387.0 & 10.241 & 0.9453 \\
\hline 393.0 & 14.196 & 0.9385 & 388.0 & 10.382 & 0.9533 \\
\hline 394.0 & 14.387 & 0.9445 & 389.0 & 10.521 & 0.9535 \\
\hline 395.0 & 14.577 & 0.9444 & 390.0 & 10.666 & 0.9501 \\
\hline 396.0 & 14.768 & 0.9455 & 391.0 & 10.807 & 0.9520 \\
\hline \multirow[t]{2}{*}{397.0} & 14.956 & 0.9470 & 392.0 & 10.950 & 0.9457 \\
\hline & & & 393.0 & 11.091 & 0.9506 \\
\hline 345.5 & 4.514 & 0.8882 & 394.0 & 11.234 & 0.9480 \\
\hline 346.0 & 4.581 & 0.9390 & 395.0 & 11.377 & 0.9446 \\
\hline 346.5 & 4.647 & 0.9508 & 396.0 & 11.520 & 0.9507 \\
\hline 347.0 & 4.713 & 0.9533 & 397.0 & 11.662 & 0.9513 \\
\hline 348.0 & 4.843 & 0.9716 & & & \\
\hline 349.0 & 4.976 & 0.9531 & 344.5 & 4.160 & 1.1976 \\
\hline 350.0 & 5.109 & 0.9459 & 345.0 & 4.212 & 1.1144 \\
\hline 351.0 & 5.241 & 0.9340 & 345.5 & 4.265 & 1.0867 \\
\hline 352.0 & 5.377 & 0.9389 & 346.0 & 4.318 & 1.0693 \\
\hline 353.0 & 5.511 & 0.9444 & 347.0 & 4.425 & 1.0433 \\
\hline 354.0 & 5.643 & 0.9277 & 348.0 & 4.531 & 1.0530 \\
\hline 355.0 & 5.779 & 0.9484 & 349.0 & 4.637 & 1.0421 \\
\hline 356.0 & 5.917 & 0.9377 & 350.0 & 4.743 & 1.0290 \\
\hline 357.0 & 6.053 & 0.9331 & 351.0 & 4.851 & 1.0022 \\
\hline
\end{tabular}


Table 1. $\mathrm{C}_{\mathrm{V}}$ for R125 (continued)

\begin{tabular}{|c|c|c|c|c|c|}
\hline $\mathrm{T}(\mathrm{K})$ & $\mathrm{P}(\mathrm{MPa})$ & $\mathrm{C}_{\mathrm{V}}(\mathrm{kJ} / \mathrm{kg}-\mathrm{K})$ & $\mathrm{T}(\mathrm{K})$ & $\mathrm{P}(\mathrm{MPa})$ & $\mathrm{C}_{\mathrm{V}}(\mathrm{kJ} / \mathrm{kg}-\mathrm{K})$ \\
\hline 352.0 & 4.960 & 0.9784 & 394.0 & 9.659 & 0.9579 \\
\hline 353.0 & 5.069 & 0.9902 & 395.0 & 9.773 & 0.9523 \\
\hline 354.0 & 5.178 & 0.9930 & 396.0 & 9.888 & 0.9563 \\
\hline 355.0 & 5.286 & 0.9877 & 397.0 & 10.001 & 0.9540 \\
\hline 356.0 & 5.396 & 0.9835 & & & \\
\hline 357.0 & 5.506 & 0.9564 & 344.5 & 4.051 & 1.2455 \\
\hline 358.0 & 5.615 & 0.9498 & 345.0 & 4.093 & 1.2273 \\
\hline 359.0 & 5.723 & 0.9367 & 345.5 & 4.136 & 1.2251 \\
\hline 360.0 & 5.835 & 0.9491 & 346.0 & 4.178 & 1.2140 \\
\hline 361.0 & 5.944 & 0.9603 & 347.0 & 4.262 & 1.1640 \\
\hline 362.0 & 6.053 & 0.9523 & 348.0 & 4.346 & 1.1357 \\
\hline 363.0 & 6.164 & 0.9744 & 349.0 & 4.432 & 1.1129 \\
\hline 364.0 & 6.274 & 0.9535 & 350.0 & 4.517 & 1.0834 \\
\hline 365.0 & 6.387 & 0.9593 & 351.0 & 4.601 & 1.0653 \\
\hline 366.0 & 6.495 & 0.9492 & 352.0 & 4.686 & 1.0457 \\
\hline 367.0 & 6.610 & 0.9546 & 353.0 & 4.773 & 1.0463 \\
\hline 368.0 & 6.720 & 0.9606 & 354.0 & 4.859 & 1.0468 \\
\hline 369.0 & 6.833 & 0.9456 & 355.0 & 4.945 & 1.0567 \\
\hline 370.0 & 6.943 & 0.9642 & 356.0 & 5.030 & 1.0468 \\
\hline 371.0 & 7.057 & 0.9424 & 357.0 & 5.117 & 1.0231 \\
\hline 372.0 & 7.170 & 0.9475 & 358.0 & 5.203 & 1.0022 \\
\hline 373.0 & 7.280 & 0.9395 & 359.0 & 5.289 & 0.9934 \\
\hline 374.0 & 7.394 & 0.9460 & 360.0 & 5.374 & 0.9937 \\
\hline 375.0 & 7.505 & 0.9438 & 361.0 & 5.460 & 0.9892 \\
\hline 376.0 & 7.620 & 0.9493 & 362.0 & 5.547 & 0.9880 \\
\hline 377.0 & 7.731 & 0.9653 & 363.0 & 5.633 & 0.9967 \\
\hline 378.0 & 7.844 & 0.9440 & 364.0 & 5.719 & 1.0069 \\
\hline 379.0 & 7.956 & 0.9563 & 365.0 & 5.804 & 0.9816 \\
\hline 380.0 & 8.071 & 0.9532 & 366.0 & 5.891 & 0.9833 \\
\hline 381.0 & 8.183 & 0.9487 & 367.0 & 5.978 & 0.9791 \\
\hline 382.0 & 8.296 & 0.9639 & 368.0 & 6.064 & 0.9651 \\
\hline 383.0 & 8.409 & 0.9542 & 369.0 & 6.148 & 0.9680 \\
\hline 384.0 & 8.523 & 0.9516 & 370.0 & 6.235 & 0.9625 \\
\hline 385.0 & 8.635 & 0.9528 & 371.0 & 6.321 & 0.9598 \\
\hline 386.0 & 8.749 & 0.9505 & 372.0 & 6.407 & 0.9635 \\
\hline 387.0 & 8.863 & 0.9595 & 373.0 & 6.492 & 0.9731 \\
\hline 388.0 & 8.977 & 0.9600 & 374.0 & 6.579 & 0.9737 \\
\hline 389.0 & 9.090 & 0.9549 & 375.0 & 6.665 & 0.9721 \\
\hline 390.0 & 9.203 & 0.9530 & 376.0 & 6.752 & 0.9730 \\
\hline 391.0 & 9.318 & 0.9539 & 377.0 & 6.839 & 0.9722 \\
\hline 392.0 & 9.432 & 0.9551 & & & \\
\hline 393.0 & 9.545 & 0.9564 & & & \\
\hline
\end{tabular}




\section{R125 EQUATION OF STATE}

The available literature data for R125 include vapor pressure, saturated liquid and vapor densities, P- $\rho$-T behavior, heat capacity, virial coefficients, and speed of sound. These were combined with the data measured in Task 1a to fit an equation of state. A complete discussion of the equation of state, the available data, and comparisons of the data with the equation of state are given in a paper to be submitted for publication in the Journal of Physical and Chemical Reference Data (Lemmon and Jacobsen 2002a). The manuscript is included here as Appendix A. A summary of the equation follows.

The equation of state for R125 has been formulated using the Helmholtz energy as the fundamental property with independent variables of density and temperature. The equation of state is given by

$$
a(\rho, T)=a^{0}(\rho, T)+a^{r}(\rho, T),
$$

where $a$ is the Helmholtz energy, $a^{0}(\rho, T)$ is the ideal gas contribution to the Helmholtz energy, and $a^{r}(\rho, T)$ is the residual Helmholtz energy. All thermodynamic properties can be calculated as derivatives of the Helmholtz energy. The functional form used for the new equation for R125 is explicit in the dimensionless Helmholtz energy, $\alpha$, using independent variables of dimensionless density and temperature. The form of this equation is

$$
\frac{a(\rho, T)}{R T}=\alpha(\delta, \tau)=\alpha^{0}(\delta, \tau)+\alpha^{r}(\delta, \tau),
$$

where $\delta=\rho / \rho_{c}, \tau=T_{c} / T$, the critical temperature $\left(T_{c}\right)$ is $339.173 \mathrm{~K}$ and the critical density $\left(\rho_{c}\right)$ is $4.779 \mathrm{~mol} / \mathrm{L}$. The ideal gas Helmholtz energy is given in a dimensionless form by

$$
\alpha^{0}=\frac{h_{0}^{0} \tau}{R T_{c}}-\frac{s_{0}^{0}}{R}-1+\ln \left(\frac{\delta \tau_{0}}{\delta_{0} \tau}\right)-\frac{\tau}{R} \int_{\tau_{0}}^{\tau} \frac{c_{p}^{0}}{\tau^{2}} \mathrm{~d} \tau+\frac{1}{R} \int_{\tau_{0}}^{\tau} \frac{c_{p}^{0}}{\tau} \mathrm{d} \tau,
$$

where $\delta_{0}=\rho_{0} / \rho_{c}, \tau_{0}=T_{c} / T_{0}$, and $\rho_{0}$ and $T_{0}$ are the density and temperature of the reference state. The equation for the ideal gas heat capacity for R125 is given by

$$
\frac{c_{p}^{0}}{R}=3.063 T^{0.1}+2.303 \frac{u_{1}^{2} \exp \left(u_{1}\right)}{\left[\exp \left(u_{1}\right)-1\right]^{2}}+5.086 \frac{u_{2}^{2} \exp \left(u_{2}\right)}{\left[\exp \left(u_{2}\right)-1\right]^{2}}+7.3 \frac{u_{3}^{2} \exp \left(u_{3}\right)}{\left[\exp \left(u_{3}\right)-1\right]^{2}},
$$

where $u_{1}$ is $314 \mathrm{~K} / T, u_{2}$ is $756 \mathrm{~K} / T, u_{3}$ is $1707 \mathrm{~K} / T$ and the gas constant, $R$, is $8.314472 \mathrm{~J} /(\mathrm{mol} \cdot \mathrm{K})$. The functional form for the residual Helmholtz energy for R125 is

$$
\alpha^{r}(\delta, \tau)=\sum_{k=1}^{5} N_{k} \delta^{i_{k}} \tau^{j_{k}}+\sum_{k=6}^{15} N_{k} \delta^{i_{k}} \tau^{j_{k}} \exp \left(-\delta^{l_{k}}\right)+\sum_{k=16}^{18} N_{k} \delta^{i_{k}} \tau^{j_{k}} \exp \left(-\delta^{l_{k}}\right) \exp \left(-\tau^{m_{k}}\right),
$$

where the coefficients and exponents are given in Table 2. The final three terms of the equation of state contain an exponential in temperature in addition to the usual density exponential. These terms, combined with theoretical constraints on the values of the exponents, result in an improved fit of the data with a minimum number of terms, as detailed in Appendix A. This represents a significant advance in the art of equations of state. 
The critical temperature and density for the equation of state were obtained by fitting saturation data at temperatures above $324 \mathrm{~K}$ to the equation

$$
\frac{\rho_{\sigma}}{\rho_{c}}-1=N_{1}\left[1-\frac{T_{\sigma}}{T_{c}}\right\rfloor \pm N_{2}\left[1-\frac{T_{\sigma}}{T_{c}}\right]^{\beta}
$$

where $N_{1}=0.98136, N_{2}=1.9125, \beta=0.33414, T_{\sigma}$ is the saturation temperature, and $\rho_{\sigma}$ is the saturation density for the liquid or the vapor. The critical density and critical temperature were fitted simultaneously with the coefficients of the equation. This equation is only valid in the critical region.

The vapor pressure can be represented with the ancillary equation

$$
\ln \left(\frac{p_{\sigma}}{p_{c}}\right)=\frac{T_{c}}{T}\left[N_{1} \theta+N_{2} \theta^{1.5}+N_{3} \theta^{2.3}+N_{4} \theta^{4.6}\right],
$$

where $N_{1}=-7.5295, N_{2}=1.9026, N_{3}=-2.2966, N_{4}=-3.4480, \theta=\left(1-T / T_{c}\right)$, and $p_{\sigma}$ is the vapor pressure. The critical pressure was determined from the equation of state at the critical temperature and density.

The resulting values of the critical temperature and density and the value of the critical pressure obtained in fitting the vapor pressure ancillary equation are

$$
\begin{aligned}
& T_{c}=339.173 \mathrm{~K}, \\
& \rho_{c}=4.779 \mathrm{~mol} / \mathrm{L}, \text { and } \\
& p_{c}=3.6177 \mathrm{MPa} .
\end{aligned}
$$

These values should be used for all property calculations using the equation of state.

The saturated liquid density is represented by the ancillary equation

$$
\frac{\rho^{\prime}}{\rho_{c}}=1+N_{1} \theta^{1 / 3}+N_{2} \theta^{0.6}+N_{3} \theta^{2.9}
$$

where $\rho^{\prime}$ is the saturated liquid density, $N_{1}=1.6684, N_{2}=0.88415$, and $N_{3}=0.44383$.

The saturated vapor density is represented by

$$
\ln \left(\frac{\rho^{\prime \prime}}{\rho_{c}}\right)=N_{1} \theta^{0.38}+N_{2} \theta^{1.22}+N_{3} \theta^{3.3}+N_{4} \theta^{6.9},
$$

where $\rho^{\prime \prime}$ is the saturated vapor density, $N_{1}=-2.8403, N_{2}=-7.2738, N_{3}=-21.890$, and $N_{4}=-58.825$. Values calculated from the equation of state using the Maxwell criteria were used in developing this equation, and deviations between the equation of state and the ancillary equation are generally less than $0.03 \%$ below $337.5 \mathrm{~K}$ and less than $0.3 \%$ elsewhere. 
The ancillary equations were helpful in the initial assessment of the data. They are presented here because they may be of utility when simple equations for the saturation boundary are needed. They are not required by the equation of state, however.

The new equation of state describes the thermodynamic surface with an uncertainty of $0.1 \%$ in density at temperatures from the triple point $(172.52 \mathrm{~K})$ to $500 \mathrm{~K}$ and pressures up to $60 \mathrm{MPa}$. In the gaseous and supercritical region, speed of sound data are represented within $0.01 \%$ at pressures below $1 \mathrm{MPa}$. The estimated uncertainty for heat capacities is $0.5 \%$ and the estimated uncertainty for the speed of sound in the liquid phase is $0.5 \%$. The saturation values can be calculated from the equation of state by application of the Maxwell criterion, which requires equal Gibbs energies and equal pressures for saturated liquid and vapor states at the same temperature. The estimated uncertainties of vapor pressures and saturated liquid densities calculated using the Maxwell criterion are $0.1 \%$ for each property, except in the critical region, and the estimated uncertainty for saturated vapor density is $0.2 \%$.

Table 2. Coefficients and exponents to the R125 equation of state.

\begin{tabular}{ccllll}
\hline \hline$k$ & \multicolumn{1}{c}{$N_{k}$} & $i_{k}$ & $j_{k}$ & $l_{k}$ & $m_{k}$ \\
\hline 1 & 5.280760 & 1 & 0.669 & & \\
2 & -8.676580 & 1 & 1.05 & & \\
3 & 0.7501127 & 1 & 2.75 & & \\
4 & 0.7590023 & 2 & 0.956 & & \\
5 & 0.01451899 & 4 & 1.00 & & \\
6 & 4.777189 & 1 & 2.00 & 1 & \\
7 & -3.330988 & 1 & 2.75 & 1 & \\
8 & 3.775673 & 2 & 2.38 & 1 & \\
9 & -2.290919 & 2 & 3.37 & 1 & \\
10 & 0.8888268 & 3 & 3.47 & 1 & \\
11 & -0.6234864 & 4 & 2.63 & 1 & \\
12 & -0.04127263 & 5 & 3.45 & 1 & \\
13 & -0.08455389 & 1 & 0.72 & 2 & \\
14 & -0.1308752 & 5 & 4.23 & 2 & \\
15 & 0.008344962 & 1 & 0.20 & 3 & \\
16 & -1.532005 & 2 & 4.5 & 2 & 1.7 \\
17 & -0.05883649 & 3 & 29.0 & 3 & 7.0 \\
18 & 0.02296658 & 5 & 24.0 & 3 & 6.0 \\
\hline
\end{tabular}




\section{MIXTURE P- $\rho$-T AND HEAT CAPACITY MEASUREMENTS}

Background. The data gaps for R410A are much more severe than those for R125. The single existing heat capacity data set for R32/125 contains only a few data points near the critical temperature, but at pressures significantly above the critical pressure. Furthermore, these data are for the 30/70 mass percent mixture. There are no heat capacity data for the 50/50 composition of R410A. There are several P- $\rho$-T data sets for R32/125, but again much of the data are for the 30/70 mass percent mixture. P- $\rho-\mathrm{T}$ data are the most important data in developing a mixture model, and thus, the temperature range of the P- $\rho$-T measurements is wider than the $\mathrm{C}_{\mathrm{v}}$ measurements. The lack of $\mathrm{P}-\rho-\mathrm{T}$ data at the R410A composition is especially pronounced for higher densities, which are the most useful in modeling. The present measurements have filled in the existing data gaps and extended well above critical.

No new measurements of the thermodynamic properties for R404A were included. Our recent literature survey of data for HFC mixtures (McLinden et al. 1998, Lemmon and McLinden 2001) revealed a number of high-quality data sets for the R125/143a/134a system and its constituent binaries (R125/143a, R125/134a, and R143a/134a) including heat capacity measurements for two of the three constituent binaries. (The available mixture data are discussed in Appendix B.) While these data are not optimal with respect to the compositions measured and coverage in the vicinity of the critical point, our modeling approach (described in Section 5) will develop a generalized mixing function for the entire class of HFC mixtures. Given the greater importance of R410A, that is where we focused our efforts.

Experimental. Cylinders of the R410A mixture were prepared gravimetrically from high-purity components. The R32 and R125 used in this work were "electronic grade" materials supplied by the Dupont Company. The analysis provided by the manufacturer stated a minimum purity of $99.99 \%$ with a water content less than $5 \mathrm{ppm}$. Our analysis by gas chromatography confirmed that it was of very high purity apart from a small amout of air in the vapor phase. The air and any other light inorganics which may have been present in the components were removed by repeatedly freezing the sample in liquid nitrogen, evacuating the vapor space, and thawing. The target compositions for the blend was $0.50 / 0.50$ mass fraction. The actual composition of the first R410A (R32/125) cylinder was 0.50001/0.49999 mass fraction. The uncertainty in composition is \pm 0.00002 mass fraction. This cylinder was used for the P- $\rho-\mathrm{T}$ and viscosity measurements.

An additional quantity of the R410A mixture was prepared gravimetrically from the same highpurity components and used for the heat capacity and thermal conductivity measurements. The actual composition of the second R410A (R32/125) sample was $0.49987 / 0.50013$ mass fraction.

Measurements of the R410A P- $\rho$-T properties were completed along 14 isochores using an isochoric (constant volume) PVT apparatus. The central element of the apparatus is a measuring cell consisting of a copper cylinder containing a cavity of approximately $28.5 \mathrm{~cm}^{3}$; the exact volume has been determined by measurements on methane and nitrogen and is corrected for temperature and pressure effects. The temperature is changed in selected increments, and the pressure is measured after equilibrium is achieved at each temperature, until the upper limit of temperature or pressure is reached.

The mixture sample is handled at all times as a low pressure gas well below the dew point pressure so that condensation and potential composition shifts are avoided. It is loaded into the apparatus by evacuating and cooling the cell. The gaseous mixture flows through a finediameter capillary and condenses to a liquid only when entering the cell; thus any composition shifts on loading are avoided. The cell is subsequently pressurized by heating. At the end of each isochore, a portion of the sample is extracted as high-pressure, single-phase fluid and 
collected in an external weighing cylinder cooled with liquid nitrogen. The weight change measured between each isochore and the final weight of the sample remaining in the cell after the last isochore provide the mass of the sample in the cell for each isochore. The volume of the sample cell together with these measured masses provide the density. The apparatus is described in detail by Magee (1996).

The P- $\rho$-T measurements cover the temperature range from 200 to $400 \mathrm{~K}$ with pressures up to $35 \mathrm{MPa}$. A special emphasis was placed on measurements near the critical state conditions, with extensive coverage of the supercritical region. The loci of the measured points on pressuretemperature coordinates are shown in Figure 2. The data themselves are given in Table 3. A comparison of these data to the mixture equation of state is given in the manuscript describing the mixture model (Appendix B of this report).

Measurements of the heat capacity of R410A were carried out using the constant-volume adiabatic calorimeter described in Section 2. For these measurements, the weight change measured between each isochore was analyzed to provide the density in addition to the heat capacity. The density and heat capacity were measured in temperature increments of $1 \mathrm{~K}$ along eight isochores (lines of constant volume) from the saturation temperature to near $400 \mathrm{~K}$ with pressures to near the upper limit pressure of the apparatus (20 MPa). Measurements on R410A were made at the temperatures and pressures shown in Figure 3. The data are given in Table 4. Good agreement was found between the fluid density measured for R410A with the calorimeter and the separate measurements made with the PVT apparatus in the region of overlap.

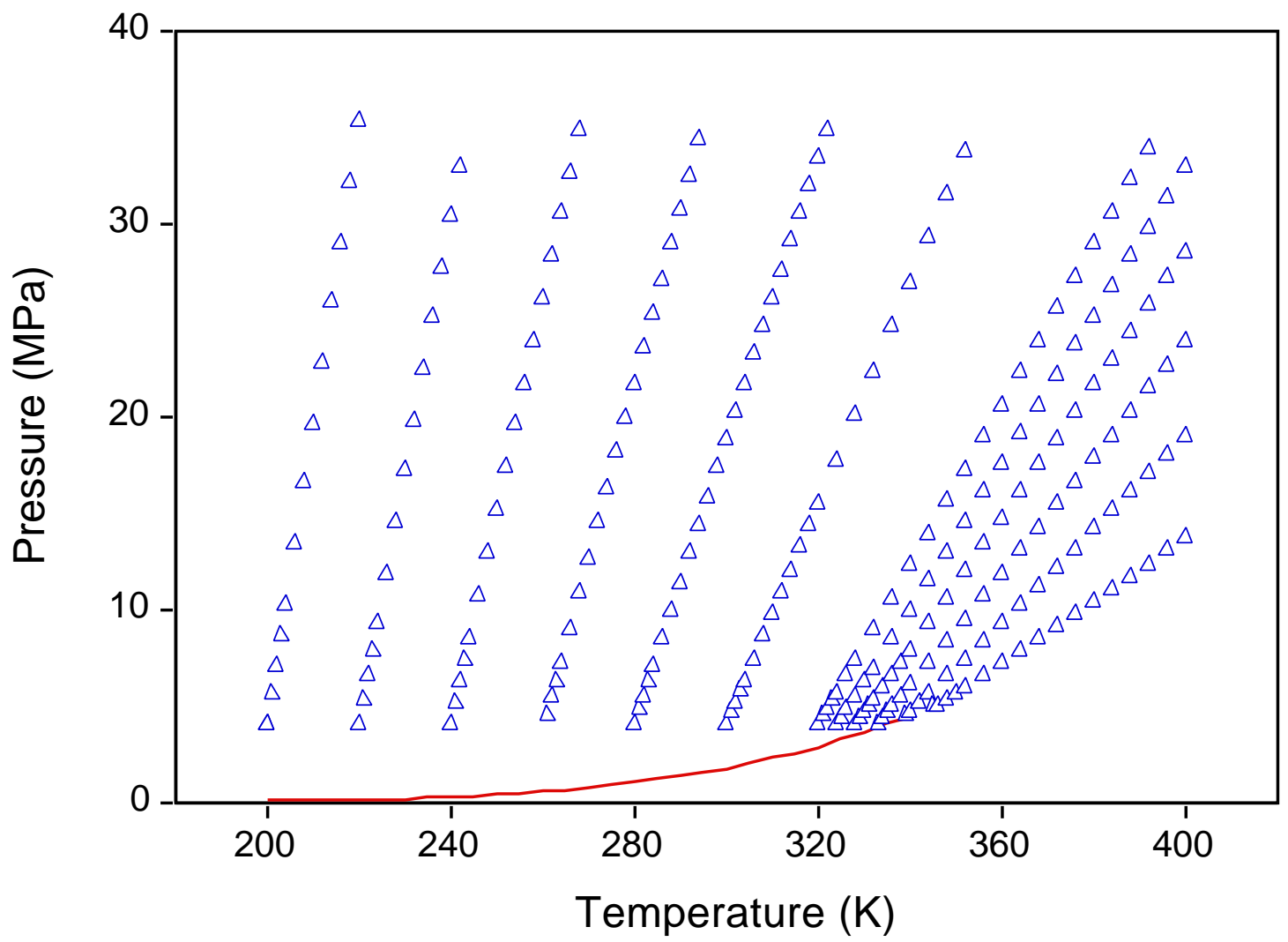

Figure 2. P- $\rho-\mathrm{T}$ (density) data for R410A. The symbols indicate measured P- $\rho-\mathrm{T}$ data points and the line indicates the bubble-point pressure. 
Table 3. Pressure-density-temperature data measured for R410A.

\begin{tabular}{|c|c|c|c|c|c|}
\hline $\mathrm{T}(\mathrm{K})$ & $\mathrm{P}(\mathrm{MPa})$ & $\rho\left(\mathrm{kg} / \mathrm{m}^{3}\right)$ & $\mathrm{T}(\mathrm{K})$ & $\mathrm{P}(\mathrm{MPa})$ & $\rho\left(\mathrm{kg} / \mathrm{m}^{3}\right)$ \\
\hline 200.001 & 4.169 & 1423.5 & 261.000 & 4.613 & 1233.6 \\
\hline 201.000 & 5.649 & 1423.2 & 262.000 & 5.505 & 1233.4 \\
\hline 202.001 & 7.188 & 1422.9 & 263.000 & 6.405 & 1233.2 \\
\hline 203.001 & 8.749 & 1422.7 & 264.000 & 7.309 & 1233.0 \\
\hline 204.000 & 10.314 & 1422.5 & 266.000 & 9.114 & 1232.7 \\
\hline 206.001 & 13.460 & 1422.1 & 267.998 & 10.927 & 1232.4 \\
\hline 208.000 & 16.606 & 1421.8 & 270.000 & 12.744 & 1232.2 \\
\hline 210.000 & 19.749 & 1421.4 & 272.000 & 14.561 & 1231.9 \\
\hline 212.000 & 22.887 & 1421.1 & 274.000 & 16.376 & 1231.7 \\
\hline 214.000 & 26.017 & 1420.8 & 276.000 & 18.189 & 1231.4 \\
\hline 216.000 & 29.136 & 1420.5 & 278.000 & 20.000 & 1231.2 \\
\hline 218.000 & 32.244 & 1420.2 & 280.001 & 21.811 & 1230.9 \\
\hline 220.000 & 35.345 & 1419.9 & 281.999 & 23.615 & 1230.7 \\
\hline \multirow[t]{2}{*}{200.000} & 4.150 & 1423.5 & 284.001 & 25.417 & 1230.4 \\
\hline & & & 286.000 & 27.221 & 1230.2 \\
\hline 220.000 & 4.148 & 1364.7 & 288.000 & 29.020 & 1230.0 \\
\hline 221.000 & 5.419 & 1364.3 & 290.000 & 30.815 & 1229.8 \\
\hline 221.999 & 6.711 & 1364.1 & 292.000 & 32.607 & 1229.5 \\
\hline 223.001 & 8.024 & 1363.9 & 294.000 & 34.394 & 1229.3 \\
\hline 224.002 & 9.344 & 1363.7 & & & \\
\hline 226.001 & 11.989 & 1363.3 & 280.000 & 4.190 & 1160.9 \\
\hline 227.998 & 14.636 & 1363.0 & 281.000 & 4.907 & 1160.7 \\
\hline 230.000 & 17.284 & 1362.7 & 282.001 & 5.636 & 1160.5 \\
\hline 232.001 & 19.926 & 1362.4 & 283.000 & 6.366 & 1160.4 \\
\hline 234.000 & 22.565 & 1362.1 & 284.000 & 7.097 & 1160.2 \\
\hline 236.001 & 25.199 & 1361.8 & 286.000 & 8.565 & 1159.9 \\
\hline 238.000 & 27.825 & 1361.5 & 288.001 & 10.033 & 1159.7 \\
\hline 240.001 & 30.445 & 1361.3 & 290.001 & 11.506 & 1159.4 \\
\hline \multirow[t]{2}{*}{242.001} & 33.058 & 1361.0 & 292.001 & 12.979 & 1159.2 \\
\hline & & & 294.000 & 14.452 & 1159.0 \\
\hline 240.002 & 4.203 & 1302.6 & 295.999 & 15.924 & 1158.7 \\
\hline 241.000 & 5.274 & 1302.4 & 298.000 & 17.397 & 1158.5 \\
\hline 242.000 & 6.364 & 1302.2 & 300.000 & 18.872 & 1158.3 \\
\hline 243.000 & 7.462 & 1302.0 & 302.000 & 20.339 & 1158.1 \\
\hline 244.000 & 8.558 & 1301.8 & 304.000 & 21.812 & 1157.9 \\
\hline 246.000 & 10.771 & 1301.5 & 306.000 & 23.282 & 1157.6 \\
\hline 248.000 & 12.980 & 1301.2 & 308.000 & 24.749 & 1157.4 \\
\hline 250.000 & 15.199 & 1300.9 & 310.000 & 26.216 & 1157.2 \\
\hline 252.000 & 17.412 & 1300.6 & 312.000 & 27.681 & 1157.0 \\
\hline 254.000 & 19.618 & 1300.3 & 314.000 & 29.145 & 1156.8 \\
\hline 256.000 & 21.823 & 1300.0 & 316.000 & 30.608 & 1156.6 \\
\hline 258.000 & 24.027 & 1299.8 & 318.000 & 32.064 & 1156.4 \\
\hline 260.000 & 26.223 & 1299.5 & 319.999 & 33.520 & 1156.2 \\
\hline 262.000 & 28.415 & 1299.3 & 321.999 & 34.976 & 1156.0 \\
\hline 264.000 & 30.603 & 1299.0 & & & \\
\hline 266.000 & 32.787 & 1298.8 & & & \\
\hline 268.000 & 34.963 & 1298.5 & & & \\
\hline
\end{tabular}


Table 3. P- $\rho-\mathrm{T}$ for R410A (continued)

\begin{tabular}{|c|c|c|c|c|c|}
\hline $\mathrm{T}(\mathrm{K})$ & $\mathrm{P}(\mathrm{MPa})$ & $\rho\left(\mathrm{kg} / \mathrm{m}^{3}\right)$ & $\mathrm{T}(\mathrm{K})$ & $\mathrm{P}(\mathrm{MPa})$ & $\rho\left(\mathrm{kg} / \mathrm{m}^{3}\right)$ \\
\hline 300.000 & 4.159 & 1073.1 & 324.001 & 4.139 & 927.8 \\
\hline 301.000 & 4.721 & 1072.9 & 325.000 & 4.500 & 927.7 \\
\hline 302.001 & 5.282 & 1072.8 & 325.999 & 4.865 & 927.6 \\
\hline 302.999 & 5.848 & 1072.6 & 328.000 & 5.598 & 927.3 \\
\hline 304.000 & 6.414 & 1072.5 & 330.000 & 6.334 & 927.1 \\
\hline 306.001 & 7.551 & 1072.2 & 332.000 & 7.075 & 926.9 \\
\hline 308.000 & 8.690 & 1072.0 & 335.999 & 8.564 & 926.6 \\
\hline 310.001 & 9.835 & 1071.8 & 340.000 & 10.063 & 926.2 \\
\hline 312.001 & 10.977 & 1071.6 & 343.999 & 11.570 & 925.9 \\
\hline 314.001 & 12.123 & 1071.3 & 348.000 & 13.085 & 925.5 \\
\hline 316.000 & 13.268 & 1071.1 & 351.999 & 14.603 & 925.2 \\
\hline 318.000 & 14.413 & 1070.9 & 356.000 & 16.127 & 924.9 \\
\hline 320.000 & 15.560 & 1070.7 & 360.000 & 17.654 & 924.5 \\
\hline 324.000 & 17.842 & 1070.4 & 364.001 & 19.182 & 924.2 \\
\hline 328.001 & 20.138 & 1070.0 & 368.001 & 20.715 & 923.9 \\
\hline 332.000 & 22.434 & 1069.6 & 372.001 & 22.245 & 923.6 \\
\hline 336.000 & 24.727 & 1069.2 & 376.000 & 23.778 & 923.3 \\
\hline 339.999 & 27.017 & 1068.8 & 380.000 & 25.311 & 923.0 \\
\hline 343.999 & 29.304 & 1068.5 & 384.001 & 26.843 & 922.6 \\
\hline 348.000 & 31.589 & 1068.1 & 388.001 & 28.376 & 922.3 \\
\hline \multirow[t]{2}{*}{351.999} & 33.869 & 1067.7 & 392.000 & 29.906 & 922.0 \\
\hline & & & 396.000 & 31.436 & 921.7 \\
\hline 320.001 & 4.191 & 958.7 & 400.000 & 32.963 & 921.4 \\
\hline 321.000 & 4.591 & 958.6 & & & \\
\hline 322.001 & 4.992 & 958.4 & 327.999 & 4.072 & 891.0 \\
\hline 323.000 & 5.393 & 958.3 & 329.000 & 4.395 & 890.9 \\
\hline 324.001 & 5.796 & 958.2 & 330.000 & 4.719 & 890.8 \\
\hline 325.999 & 6.606 & 958.0 & 331.000 & 5.046 & 890.6 \\
\hline 328.000 & 7.420 & 957.8 & 332.001 & 5.372 & 890.5 \\
\hline 332.000 & 9.053 & 957.4 & 333.999 & 6.029 & 890.3 \\
\hline 336.000 & 10.696 & 957.0 & 336.001 & 6.690 & 890.2 \\
\hline 339.999 & 12.347 & 956.7 & 338.000 & 7.359 & 890.0 \\
\hline 343.999 & 14.003 & 956.3 & 339.999 & 8.023 & 889.8 \\
\hline 348.000 & 15.663 & 956.0 & 344.000 & 9.366 & 889.5 \\
\hline 352.000 & 17.326 & 955.7 & 347.999 & 10.719 & 889.1 \\
\hline 356.001 & 18.994 & 955.3 & 351.999 & 12.079 & 888.8 \\
\hline 360.000 & 20.662 & 955.0 & 356.001 & 13.444 & 888.5 \\
\hline 364.000 & 22.330 & 954.7 & 359.999 & 14.816 & 888.2 \\
\hline 368.001 & 23.999 & 954.3 & 364.001 & 16.190 & 887.9 \\
\hline 372.000 & 25.667 & 954.0 & 368.000 & 17.569 & 887.6 \\
\hline 376.000 & 27.336 & 953.7 & 372.000 & 18.949 & 887.3 \\
\hline 380.000 & 29.002 & 953.3 & 376.000 & 20.331 & 887.0 \\
\hline 384.000 & 30.668 & 953.0 & 380.001 & 21.715 & 886.7 \\
\hline 388.000 & 32.333 & 952.7 & 384.000 & 23.100 & 886.4 \\
\hline \multirow[t]{4}{*}{392.001} & 33.994 & 952.3 & 388.000 & 24.486 & 886.1 \\
\hline & & & 392.001 & 25.871 & 885.7 \\
\hline & & & 395.999 & 27.255 & 885.4 \\
\hline & & & 400.000 & 28.641 & 885.1 \\
\hline
\end{tabular}


Table 3. P- $\rho-\mathrm{T}$ for R410A (continued)

\begin{tabular}{|c|c|c|c|c|c|}
\hline $\mathrm{T}(\mathrm{K})$ & $\mathrm{P}(\mathrm{MPa})$ & $\rho\left(\mathrm{kg} / \mathrm{m}^{3}\right)$ & $\mathrm{T}(\mathrm{K})$ & $\mathrm{P}(\mathrm{MPa})$ & $\rho\left(\mathrm{kg} / \mathrm{m}^{3}\right)$ \\
\hline 333.001 & 4.203 & 840.7 & 344.999 & 5.018 & 625.3 \\
\hline 334.000 & 4.477 & 840.6 & 346.000 & 5.162 & 625.3 \\
\hline 335.000 & 4.755 & 840.5 & 347.999 & 5.454 & 625.2 \\
\hline 336.000 & 5.032 & 840.4 & 349.999 & 5.752 & 625.1 \\
\hline 338.000 & 5.592 & 840.2 & 352.000 & 6.054 & 624.9 \\
\hline 340.001 & 6.157 & 840.0 & 356.000 & 6.668 & 624.7 \\
\hline 344.001 & 7.297 & 839.7 & 360.000 & 7.292 & 624.5 \\
\hline 348.000 & 8.451 & 839.4 & 364.001 & 7.922 & 624.3 \\
\hline 351.998 & 9.614 & 839.1 & 368.000 & 8.560 & 624.1 \\
\hline 356.000 & 10.785 & 838.8 & 372.000 & 9.202 & 623.9 \\
\hline 360.000 & 11.964 & 838.5 & 376.000 & 9.847 & 623.7 \\
\hline 364.000 & 13.148 & 838.2 & 380.001 & 10.497 & 623.4 \\
\hline 367.999 & 14.338 & 837.9 & 384.001 & 11.149 & 623.2 \\
\hline 372.000 & 15.532 & 837.6 & 387.999 & 11.804 & 623.0 \\
\hline 376.001 & 16.727 & 837.3 & 392.000 & 12.460 & 622.8 \\
\hline 380.000 & 17.928 & 837.0 & 396.000 & 13.118 & 622.6 \\
\hline 384.000 & 19.129 & 836.7 & 400.000 & 13.778 & 622.4 \\
\hline 388.000 & 20.332 & 836.5 & & & \\
\hline 392.000 & 21.536 & 836.2 & & & \\
\hline 396.001 & 22.741 & 835.9 & & & \\
\hline 400.001 & 23.946 & 835.6 & & & \\
\hline 339.000 & 4.584 & 767.8 & & & \\
\hline 339.999 & 4.803 & 767.7 & & & \\
\hline 342.000 & 5.245 & 767.6 & & & \\
\hline 344.001 & 5.694 & 767.4 & & & \\
\hline 348.001 & 6.604 & 767.1 & & & \\
\hline 352.000 & 7.529 & 766.8 & & & \\
\hline 356.000 & 8.466 & 766.5 & & & \\
\hline 359.998 & 9.412 & 766.3 & & & \\
\hline 364.001 & 10.366 & 766.0 & & & \\
\hline 368.000 & 11.325 & 765.7 & & & \\
\hline 372.001 & 12.290 & 765.5 & & & \\
\hline 376.001 & 13.260 & 765.2 & & & \\
\hline 380.000 & 14.235 & 765.0 & & & \\
\hline 384.000 & 15.211 & 764.7 & & & \\
\hline 388.000 & 16.190 & 764.4 & & & \\
\hline 392.001 & 17.172 & 764.2 & & & \\
\hline 396.000 & 18.155 & 763.9 & & & \\
\hline 400.000 & 19.140 & 763.7 & & & \\
\hline
\end{tabular}




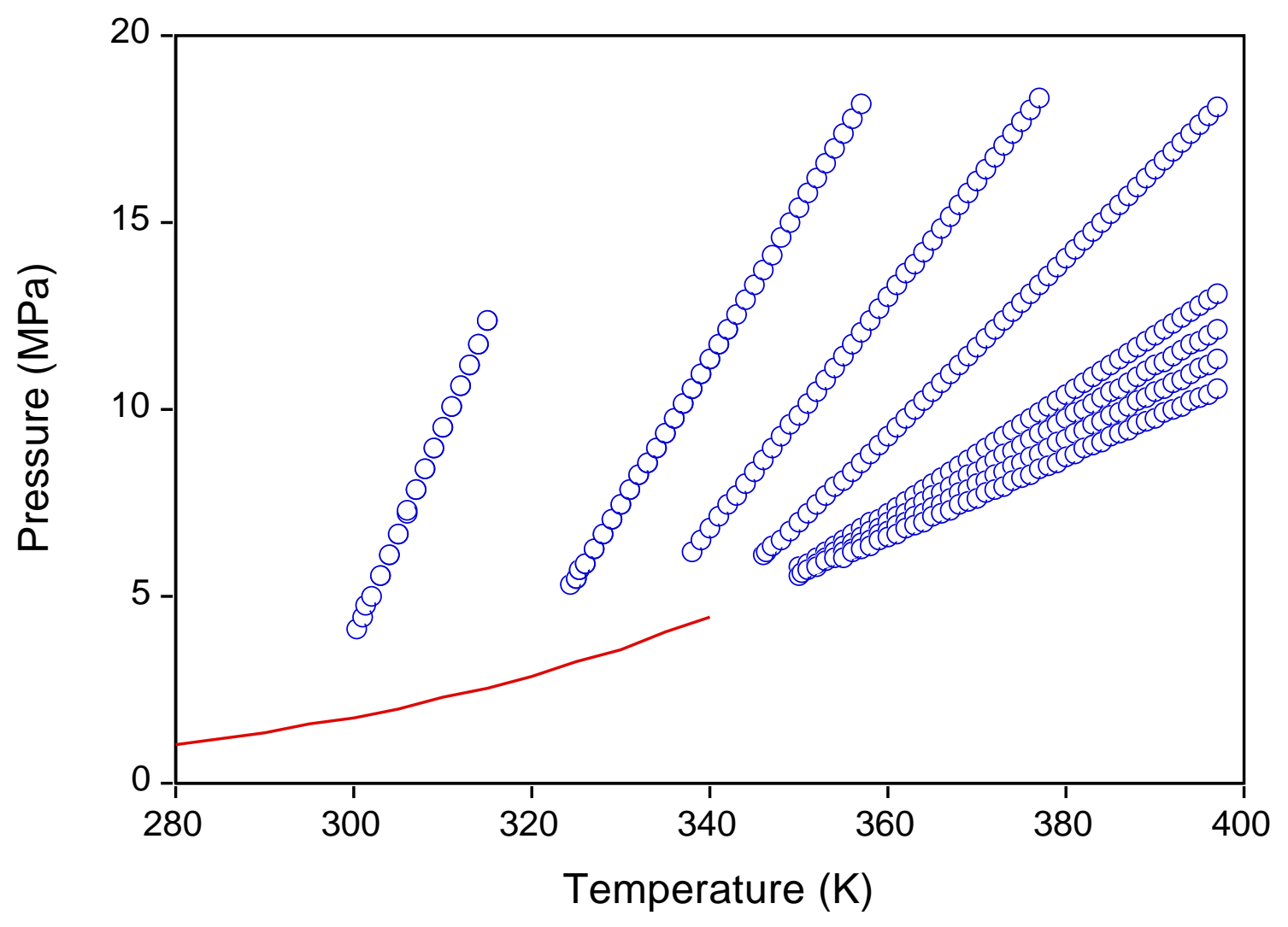

Figure 3. Isochoric heat capacity data for R410A. The symbols indicate measured $\mathrm{C}_{\mathrm{V}}$ data points and the line indicates the bubble-point pressure. P- $\rho-\mathrm{T}$ (density) and $\mathrm{C}_{\mathrm{V}}$ data were simultaneously measured at each point. 
Table 4. Isochoric heat capacity and P- $\rho-T$ data measured for R410A.

\begin{tabular}{|c|c|c|c|c|c|c|c|}
\hline \multirow{2}{*}{$\begin{array}{l}\mathrm{T}(\mathrm{K}) \\
303.0\end{array}$} & \multirow{2}{*}{$\begin{array}{c}\mathrm{P}(\mathrm{MPa}) \\
5.537\end{array}$} & \multicolumn{2}{|c|}{$\rho\left(\mathrm{kg} / \mathrm{m}^{3}\right) \mathrm{C}_{\mathrm{V}}(\mathrm{kJ} / \mathrm{kg}-\mathrm{K})$} & \multirow{2}{*}{$\begin{array}{l}\mathrm{T}(\mathrm{K}) \\
342.0\end{array}$} & \multirow{2}{*}{$\begin{array}{c}\mathrm{P}(\mathrm{MPa}) \\
12.139\end{array}$} & \multicolumn{2}{|c|}{$\rho\left(\mathrm{kg} / \mathrm{m}^{3}\right) \mathrm{C}_{\mathrm{V}}(\mathrm{kJ} / \mathrm{kg}-\mathrm{K})$} \\
\hline & & 1070.8 & 0.931 & & & 944.5 & 0.943 \\
\hline 304.0 & 6.101 & 1070.7 & 0.932 & & & & \\
\hline 305.0 & 6.664 & 1070.6 & 0.930 & 325.0 & 5.448 & 946.2 & 0.976 \\
\hline 306.0 & 7.228 & 1070.4 & 0.932 & 325.5 & 5.642 & 946.1 & 0.970 \\
\hline 307.0 & 7.791 & 1070.3 & 0.933 & 326.0 & 5.837 & 946.1 & 0.975 \\
\hline 308.0 & 8.355 & 1070.2 & 0.929 & 327.0 & 6.228 & 946.0 & 0.963 \\
\hline 309.0 & 8.920 & 1070.0 & 0.933 & 328.0 & 6.618 & 945.9 & 0.970 \\
\hline 310.0 & 9.485 & 1069.9 & 0.931 & 329.0 & 7.007 & 945.8 & 0.972 \\
\hline 311.0 & 10.049 & 1069.8 & 0.929 & 330.0 & 7.400 & 945.7 & 0.969 \\
\hline 312.0 & 10.612 & 1069.6 & 0.928 & 331.0 & 7.793 & 945.6 & 0.971 \\
\hline 313.0 & 11.179 & 1069.5 & 0.925 & 332.0 & 8.184 & 945.5 & 0.969 \\
\hline 314.0 & 11.744 & 1069.4 & 0.925 & 333.0 & 8.577 & 945.4 & 0.968 \\
\hline 315.0 & 12.310 & 1069.2 & 0.923 & 334.0 & 8.971 & 945.3 & 0.966 \\
\hline 300.5 & 4.133 & 1071.2 & 0.931 & 335.0 & 9.367 & 945.2 & 0.967 \\
\hline 301.0 & 4.415 & 1071.1 & 0.931 & 336.0 & 9.761 & 945.1 & 0.968 \\
\hline 301.5 & 4.696 & 1071.0 & 0.930 & 337.0 & 10.156 & 945.0 & 0.968 \\
\hline 302.0 & 4.978 & 1071.0 & 0.930 & 338.0 & 10.553 & 944.9 & 0.971 \\
\hline 303.0 & 5.539 & 1070.8 & 0.927 & 339.0 & 10.950 & 944.8 & 0.967 \\
\hline 304.0 & 6.102 & 1070.7 & 0.929 & 340.0 & 11.347 & 944.7 & 0.969 \\
\hline 305.0 & 6.666 & 1070.6 & 0.930 & 341.0 & 11.743 & 944.6 & 0.971 \\
\hline 306.0 & 7.230 & 1070.4 & 0.930 & 342.0 & 12.141 & 944.5 & 0.964 \\
\hline 307.0 & 7.791 & 1070.3 & 0.931 & 343.0 & 12.539 & 944.4 & 0.962 \\
\hline 308.0 & 8.355 & 1070.2 & 0.931 & 344.0 & 12.938 & 944.3 & 0.962 \\
\hline 309.0 & 8.920 & 1070.0 & 0.931 & 345.0 & 13.334 & 944.3 & 0.954 \\
\hline 310.0 & 9.486 & 1069.9 & 0.930 & 346.0 & 13.732 & 944.2 & 0.952 \\
\hline 311.0 & 10.048 & 1069.8 & 0.930 & 347.0 & 14.132 & 944.1 & 0.957 \\
\hline 312.0 & 10.612 & 1069.6 & 0.925 & 348.0 & 14.532 & 944.0 & 0.952 \\
\hline 313.0 & 11.179 & 1069.5 & 0.921 & 349.0 & 14.930 & 943.9 & 0.953 \\
\hline 314.0 & 11.746 & 1069.4 & 0.921 & 350.0 & 15.328 & 943.8 & 0.952 \\
\hline \multirow[t]{2}{*}{315.0} & 12.309 & 1069.2 & 0.915 & 351.0 & 15.729 & 943.7 & 0.947 \\
\hline & & & & 352.0 & 16.130 & 943.6 & 0.944 \\
\hline 324.5 & 5.255 & 946.2 & 0.950 & 353.0 & 16.529 & 943.5 & 0.941 \\
\hline 325.0 & 5.449 & 946.2 & 0.948 & 354.0 & 16.928 & 943.4 & 0.942 \\
\hline 325.5 & 5.643 & 946.1 & 0.951 & 355.0 & 17.329 & 943.3 & 0.940 \\
\hline 326.0 & 5.838 & 946.1 & 0.954 & 356.0 & 17.729 & 943.2 & 0.940 \\
\hline 327.0 & 6.227 & 946.0 & 0.957 & 357.0 & 18.129 & 943.1 & 0.941 \\
\hline 328.0 & 6.616 & 945.9 & 0.954 & & & & \\
\hline 329.0 & 7.006 & 945.8 & 0.954 & 338.0 & 6.180 & 859.9 & 0.996 \\
\hline 330.0 & 7.400 & 945.7 & 0.956 & 339.0 & 6.484 & 859.8 & 1.000 \\
\hline 331.0 & 7.793 & 945.6 & 0.955 & 340.0 & 6.784 & 859.8 & 0.994 \\
\hline 332.0 & 8.184 & 945.5 & 0.955 & 341.0 & 7.085 & 859.7 & 0.994 \\
\hline 333.0 & 8.576 & 945.4 & 0.951 & 342.0 & 7.390 & 859.6 & 0.989 \\
\hline 334.0 & 8.972 & 945.3 & 0.948 & 343.0 & 7.697 & 859.5 & 0.987 \\
\hline 335.0 & 9.368 & 945.2 & 0.952 & 344.0 & 8.000 & 859.5 & 0.993 \\
\hline 336.0 & 9.762 & 945.1 & 0.953 & 345.0 & 8.305 & 859.4 & 0.989 \\
\hline 337.0 & 10.156 & 945.0 & 0.950 & 346.0 & 8.612 & 859.3 & 0.987 \\
\hline 338.0 & 10.552 & 944.9 & 0.948 & 347.0 & 8.920 & 859.2 & 0.986 \\
\hline 339.0 & 10.950 & 944.8 & 0.944 & 348.0 & 9.228 & 859.2 & 0.982 \\
\hline 340.0 & 11.346 & 944.7 & 0.942 & 349.0 & 9.535 & 859.1 & 0.986 \\
\hline 341.0 & 11.742 & 944.6 & 0.947 & 350.0 & 9.844 & 859.0 & 0.985 \\
\hline
\end{tabular}


Table 4. R410A C $\mathrm{V}_{\mathrm{V}}$ and P- $\rho-\mathrm{T}$ (continued)

\begin{tabular}{|c|c|c|c|c|c|c|c|}
\hline \multirow{2}{*}{$\begin{array}{l}\mathrm{T}(\mathrm{K}) \\
351.0\end{array}$} & \multirow{2}{*}{$\begin{array}{c}\mathrm{P}(\mathrm{MPa}) \\
10.153\end{array}$} & \multicolumn{2}{|c|}{$\rho\left(\mathrm{kg} / \mathrm{m}^{3}\right) \mathrm{C}_{\mathrm{V}}(\mathrm{kJ} / \mathrm{kg}-\mathrm{K})$} & \multirow{2}{*}{$\begin{array}{l}\mathrm{T}(\mathrm{K}) \\
367.0\end{array}$} & \multirow{2}{*}{$\begin{array}{c}\mathrm{P}(\mathrm{MPa}) \\
10.898\end{array}$} & \multicolumn{2}{|c|}{$\rho\left(\mathrm{kg} / \mathrm{m}^{3}\right) \mathrm{C}_{\mathrm{V}}(\mathrm{kJ} / \mathrm{kg}-\mathrm{K})$} \\
\hline & & 858.9 & 0.981 & & & 759.9 & 0.975 \\
\hline 352.0 & 10.463 & 858.9 & 0.983 & 368.0 & 11.136 & 759.9 & 0.973 \\
\hline 353.0 & 10.773 & 858.8 & 0.976 & 369.0 & 11.372 & 759.8 & 0.979 \\
\hline 354.0 & 11.082 & 858.7 & 0.977 & 370.0 & 11.607 & 759.8 & 0.979 \\
\hline 355.0 & 11.393 & 858.6 & 0.976 & 371.0 & 11.844 & 759.7 & 0.980 \\
\hline 356.0 & 11.705 & 858.5 & 0.977 & 372.0 & 12.082 & 759.7 & 0.980 \\
\hline 357.0 & 12.016 & 858.5 & 0.976 & 373.0 & 12.319 & 759.6 & 0.975 \\
\hline 358.0 & 12.327 & 858.4 & 0.972 & 374.0 & 12.557 & 759.5 & 0.982 \\
\hline 359.0 & 12.640 & 858.3 & 0.972 & 375.0 & 12.795 & 759.5 & 0.979 \\
\hline 360.0 & 12.955 & 858.2 & 0.963 & 376.0 & 13.034 & 759.4 & 0.975 \\
\hline 361.0 & 13.266 & 858.2 & 0.963 & 377.0 & 13.271 & 759.4 & 0.980 \\
\hline 362.0 & 13.579 & 858.1 & 0.956 & 378.0 & 13.510 & 759.3 & 0.975 \\
\hline 363.0 & 13.892 & 858.0 & 0.948 & 379.0 & 13.749 & 759.2 & 0.977 \\
\hline 364.0 & 14.208 & 857.9 & 0.951 & 380.0 & 13.989 & 759.2 & 0.981 \\
\hline 365.0 & 14.521 & 857.9 & 0.941 & 381.0 & 14.227 & 759.1 & 0.984 \\
\hline 366.0 & 14.834 & 857.8 & 0.942 & 382.0 & 14.466 & 759.1 & 0.985 \\
\hline 367.0 & 15.149 & 857.7 & 0.947 & 383.0 & 14.707 & 759.0 & 0.988 \\
\hline 368.0 & 15.465 & 857.6 & 0.944 & 384.0 & 14.946 & 758.9 & 0.992 \\
\hline 369.0 & 15.779 & 857.6 & 0.952 & 385.0 & 15.186 & 758.9 & 0.988 \\
\hline 370.0 & 16.093 & 857.5 & 0.951 & 386.0 & 15.424 & 758.8 & 0.986 \\
\hline 371.0 & 16.407 & 857.4 & 0.946 & 387.0 & 15.667 & 758.8 & 0.983 \\
\hline 372.0 & 16.724 & 857.3 & 0.950 & 388.0 & 15.907 & 758.7 & 0.979 \\
\hline 373.0 & 17.039 & 857.3 & 0.947 & 389.0 & 16.146 & 758.6 & 0.979 \\
\hline 374.0 & 17.354 & 857.2 & 0.951 & 390.0 & 16.386 & 758.6 & 0.981 \\
\hline 375.0 & 17.669 & 857.1 & 0.955 & 391.0 & 16.628 & 758.5 & 0.980 \\
\hline 376.0 & 17.987 & 857.0 & 0.953 & 392.0 & 16.869 & 758.5 & 0.978 \\
\hline \multirow[t]{2}{*}{377.0} & 18.302 & 856.9 & 0.960 & 393.0 & 17.108 & 758.4 & 0.979 \\
\hline & & & & 394.0 & 17.350 & 758.4 & 0.978 \\
\hline 346.0 & 6.059 & 761.1 & 1.027 & 395.0 & 17.592 & 758.3 & 0.977 \\
\hline 346.5 & 6.171 & 761.1 & 1.031 & 396.0 & 17.833 & 758.2 & 0.984 \\
\hline 347.0 & 6.284 & 761.1 & 1.034 & 397.0 & 18.073 & 758.2 & 0.983 \\
\hline 348.0 & 6.508 & 761.0 & 1.022 & & & & \\
\hline 349.0 & 6.732 & 761.0 & 1.021 & 350.0 & 5.722 & 615.0 & 1.178 \\
\hline 350.0 & 6.958 & 760.9 & 1.009 & 351.0 & 5.869 & 615.0 & 1.145 \\
\hline 351.0 & 7.186 & 760.9 & 1.004 & 352.0 & 6.016 & 614.9 & 1.133 \\
\hline 352.0 & 7.412 & 760.8 & 0.993 & 353.0 & 6.163 & 614.9 & 1.095 \\
\hline 353.0 & 7.641 & 760.7 & 0.998 & 354.0 & 6.311 & 614.9 & 1.090 \\
\hline 354.0 & 7.871 & 760.7 & 0.999 & 355.0 & 6.462 & 614.9 & 1.064 \\
\hline 355.0 & 8.101 & 760.6 & 0.992 & 356.0 & 6.613 & 614.8 & 1.048 \\
\hline 356.0 & 8.331 & 760.6 & 0.995 & 357.0 & 6.762 & 614.8 & 1.044 \\
\hline 357.0 & 8.564 & 760.5 & 0.988 & 358.0 & 6.914 & 614.8 & 1.050 \\
\hline 358.0 & 8.794 & 760.5 & 0.980 & 359.0 & 7.065 & 614.7 & 1.012 \\
\hline 359.0 & 9.028 & 760.4 & 0.981 & 360.0 & 7.218 & 614.7 & 1.011 \\
\hline 360.0 & 9.260 & 760.4 & 0.981 & 361.0 & 7.370 & 614.7 & 1.016 \\
\hline 361.0 & 9.493 & 760.3 & 0.981 & 362.0 & 7.523 & 614.6 & 0.989 \\
\hline 362.0 & 9.725 & 760.2 & 0.982 & 363.0 & 7.675 & 614.6 & 1.005 \\
\hline 363.0 & 9.960 & 760.2 & 0.979 & 364.0 & 7.830 & 614.6 & 1.004 \\
\hline 364.0 & 10.195 & 760.1 & 0.980 & 365.0 & 7.985 & 614.5 & 1.000 \\
\hline 365.0 & 10.429 & 760.1 & 0.975 & 366.0 & 8.138 & 614.5 & 1.006 \\
\hline 366.0 & 10.663 & 760.0 & 0.972 & 367.0 & 8.294 & 614.5 & 1.014 \\
\hline
\end{tabular}


Table 4. R410A $\mathrm{C}_{\mathrm{V}}$ and P- $\rho-\mathrm{T}$ (continued)

\begin{tabular}{|c|c|c|c|c|c|c|c|}
\hline $\mathrm{T}(\mathrm{K})$ & $\mathrm{P}(\mathrm{MPa})$ & $\rho\left(\mathrm{kg} / \mathrm{m}^{3}\right)$ & $\mathrm{C}_{\mathrm{V}}(\mathrm{kJ} / \mathrm{kg}-\mathrm{K})$ & $\mathrm{T}(\mathrm{K})$ & $\mathrm{P}(\mathrm{MPa})$ & \multicolumn{2}{|c|}{$\rho\left(\mathrm{kg} / \mathrm{m}^{3}\right) \mathrm{C}_{\mathrm{V}}(\mathrm{kJ} / \mathrm{kg}-\mathrm{K})$} \\
\hline 368.0 & 8.450 & 614.4 & 1.012 & 371.0 & 8.462 & 571.0 & 1.035 \\
\hline 369.0 & 8.605 & 614.4 & 1.010 & 372.0 & 8.600 & 570.9 & 1.032 \\
\hline 370.0 & 8.761 & 614.4 & 1.008 & 373.0 & 8.738 & 570.9 & 1.029 \\
\hline 371.0 & 8.918 & 614.3 & 0.996 & 374.0 & 8.878 & 570.9 & 1.018 \\
\hline 372.0 & 9.075 & 614.3 & 0.997 & 375.0 & 9.018 & 570.8 & 1.022 \\
\hline 373.0 & 9.230 & 614.2 & 0.985 & 376.0 & 9.157 & 570.8 & 1.026 \\
\hline 374.0 & 9.387 & 614.2 & 0.987 & 377.0 & 9.296 & 570.8 & 1.036 \\
\hline 375.0 & 9.544 & 614.2 & 0.987 & 378.0 & 9.435 & 570.7 & 1.031 \\
\hline 376.0 & 9.703 & 614.1 & 0.992 & 379.0 & 9.576 & 570.7 & 1.027 \\
\hline 377.0 & 9.859 & 614.1 & 1.001 & 380.0 & 9.716 & 570.7 & 1.025 \\
\hline 378.0 & 10.016 & 614.1 & 0.995 & 381.0 & 9.856 & 570.6 & 1.018 \\
\hline 379.0 & 10.175 & 614.0 & 1.004 & 382.0 & 9.996 & 570.6 & 1.031 \\
\hline 380.0 & 10.334 & 614.0 & 0.996 & 383.0 & 10.137 & 570.6 & 1.028 \\
\hline 381.0 & 10.491 & 613.9 & 1.001 & 384.0 & 10.277 & 570.5 & 1.028 \\
\hline 382.0 & 10.649 & 613.9 & 1.011 & 385.0 & 10.417 & 570.5 & 1.033 \\
\hline 383.0 & 10.808 & 613.9 & 1.001 & 386.0 & 10.559 & 570.5 & 1.031 \\
\hline 384.0 & 10.967 & 613.8 & 1.006 & 387.0 & 10.699 & 570.4 & 1.033 \\
\hline 385.0 & 11.125 & 613.8 & 1.005 & 388.0 & 10.841 & 570.4 & 1.043 \\
\hline 386.0 & 11.285 & 613.7 & 1.004 & 389.0 & 10.981 & 570.4 & 1.043 \\
\hline 387.0 & 11.443 & 613.7 & 1.005 & 390.0 & 11.122 & 570.3 & 1.036 \\
\hline 388.0 & 11.602 & 613.7 & 1.009 & 391.0 & 11.263 & 570.3 & 1.040 \\
\hline 389.0 & 11.762 & 613.6 & 1.011 & 392.0 & 11.406 & 570.3 & 1.033 \\
\hline 390.0 & 11.921 & 613.6 & 1.006 & 393.0 & 11.546 & 570.2 & 1.033 \\
\hline 391.0 & 12.080 & 613.5 & 1.016 & 394.0 & 11.688 & 570.2 & 1.048 \\
\hline 392.0 & 12.241 & 613.5 & 1.011 & 395.0 & 11.830 & 570.2 & 1.040 \\
\hline 393.0 & 12.401 & 613.5 & 1.008 & 396.0 & 11.972 & 570.1 & 1.042 \\
\hline 394.0 & 12.559 & 613.4 & 1.016 & 397.0 & 12.112 & 570.1 & 1.044 \\
\hline 395.0 & 12.720 & 613.4 & 1.008 & & & & \\
\hline 396.0 & 12.880 & 613.3 & 1.009 & 350.0 & 5.544 & 525.9 & 1.364 \\
\hline \multirow[t]{2}{*}{397.0} & 13.041 & 613.3 & 1.011 & 350.5 & 5.603 & 525.9 & 1.307 \\
\hline & & & & 351.0 & 5.663 & 525.9 & 1.332 \\
\hline 352.0 & 5.879 & 571.5 & 1.317 & 352.0 & 5.780 & 525.8 & 1.288 \\
\hline 353.0 & 6.011 & 571.4 & 1.260 & 353.0 & 5.898 & 525.8 & 1.232 \\
\hline 354.0 & 6.143 & 571.4 & 1.200 & 354.0 & 6.017 & 525.8 & 1.233 \\
\hline 355.0 & 6.277 & 571.4 & 1.183 & 355.0 & 6.137 & 525.8 & 1.160 \\
\hline 356.0 & 6.411 & 571.4 & 1.151 & 356.0 & 6.256 & 525.8 & 1.168 \\
\hline 357.0 & 6.545 & 571.3 & 1.135 & 357.0 & 6.376 & 525.8 & 1.149 \\
\hline 358.0 & 6.679 & 571.3 & 1.131 & 358.0 & 6.496 & 525.8 & 1.129 \\
\hline 359.0 & 6.814 & 571.3 & 1.106 & 359.0 & 6.617 & 525.7 & 1.129 \\
\hline 360.0 & 6.950 & 571.3 & 1.104 & 360.0 & 6.738 & 525.7 & 1.118 \\
\hline 361.0 & 7.085 & 571.2 & 1.089 & 361.0 & 6.858 & 525.7 & 1.102 \\
\hline 362.0 & 7.221 & 571.2 & 1.092 & 362.0 & 6.980 & 525.7 & 1.095 \\
\hline 363.0 & 7.358 & 571.2 & 1.090 & 363.0 & 7.101 & 525.7 & 1.084 \\
\hline 364.0 & 7.496 & 571.2 & 1.087 & 364.0 & 7.223 & 525.7 & 1.072 \\
\hline 365.0 & 7.632 & 571.1 & 1.076 & 365.0 & 7.344 & 525.6 & 1.075 \\
\hline 366.0 & 7.769 & 571.1 & 1.065 & 366.0 & 7.465 & 525.6 & 1.059 \\
\hline 367.0 & 7.908 & 571.1 & 1.062 & 367.0 & 7.587 & 525.6 & 1.067 \\
\hline 368.0 & 8.047 & 571.1 & 1.040 & 368.0 & 7.709 & 525.6 & 1.054 \\
\hline 369.0 & 8.183 & 571.0 & 1.040 & 369.0 & 7.831 & 525.5 & 1.054 \\
\hline 370.0 & 8.323 & 571.0 & 1.043 & 370.0 & 7.953 & 525.5 & 1.058 \\
\hline
\end{tabular}


Table 4. R410A C $\mathrm{V}_{\mathrm{V}}$ and P- $\rho-\mathrm{T}$ (continued)

\begin{tabular}{|c|c|c|c|c|c|c|c|}
\hline $\mathrm{T}(\mathrm{K})$ & $\mathrm{P}(\mathrm{MPa})$ & \multicolumn{2}{|c|}{$\rho\left(\mathrm{kg} / \mathrm{m}^{3}\right) \mathrm{C}_{\mathrm{V}}(\mathrm{kJ} / \mathrm{kg}-\mathrm{K})$} & \multirow{2}{*}{$\begin{array}{l}\mathrm{T}(\mathrm{K}) \\
377.0\end{array}$} & \multirow{2}{*}{$\begin{array}{c}\mathrm{P}(\mathrm{MPa}) \\
8.361\end{array}$} & \multicolumn{2}{|c|}{$\rho\left(\mathrm{kg} / \mathrm{m}^{3}\right) \mathrm{C}_{\mathrm{V}}(\mathrm{kJ} / \mathrm{kg}-\mathrm{K})$} \\
\hline 371.0 & 8.076 & 525.5 & 1.045 & & & 475.6 & 1.057 \\
\hline 372.0 & 8.199 & 525.5 & 1.058 & 378.0 & 8.468 & 475.5 & 1.064 \\
\hline 373.0 & 8.321 & 525.4 & 1.038 & 379.0 & 8.576 & 475.5 & 1.057 \\
\hline 374.0 & 8.444 & 525.4 & 1.043 & 380.0 & 8.683 & 475.5 & 1.062 \\
\hline 375.0 & 8.567 & 525.4 & 1.039 & 381.0 & 8.790 & 475.5 & 1.070 \\
\hline 376.0 & 8.691 & 525.4 & 1.035 & 382.0 & 8.898 & 475.4 & 1.066 \\
\hline 377.0 & 8.813 & 525.3 & 1.042 & 383.0 & 9.005 & 475.4 & 1.063 \\
\hline 378.0 & 8.937 & 525.3 & 1.037 & 384.0 & 9.112 & 475.4 & 1.065 \\
\hline 379.0 & 9.060 & 525.3 & 1.043 & 385.0 & 9.219 & 475.4 & 1.057 \\
\hline 380.0 & 9.184 & 525.3 & 1.042 & 386.0 & 9.326 & 475.4 & 1.055 \\
\hline 381.0 & 9.306 & 525.2 & 1.038 & 387.0 & 9.434 & 475.3 & 1.062 \\
\hline 382.0 & 9.431 & 525.2 & 1.050 & 388.0 & 9.541 & 475.3 & 1.058 \\
\hline 383.0 & 9.555 & 525.2 & 1.045 & 389.0 & 9.648 & 475.3 & 1.058 \\
\hline 384.0 & 9.680 & 525.1 & 1.051 & 390.0 & 9.754 & 475.2 & 1.066 \\
\hline 385.0 & 9.802 & 525.1 & 1.051 & 391.0 & 9.863 & 475.2 & 1.064 \\
\hline 386.0 & 9.926 & 525.1 & 1.032 & 392.0 & 9.970 & 475.2 & 1.069 \\
\hline 387.0 & 10.050 & 525.0 & 1.030 & 393.0 & 10.077 & 475.2 & 1.074 \\
\hline 388.0 & 10.175 & 525.0 & 1.027 & 394.0 & 10.185 & 475.1 & 1.077 \\
\hline 389.0 & 10.299 & 525.0 & 1.039 & 395.0 & 10.292 & 475.1 & 1.075 \\
\hline 390.0 & 10.422 & 525.0 & 1.042 & 396.0 & 10.400 & 475.1 & 1.075 \\
\hline 391.0 & 10.546 & 524.9 & 1.053 & 397.0 & 10.507 & 475.1 & 1.072 \\
\hline 392.0 & 10.671 & 524.9 & 1.050 & & & & \\
\hline 393.0 & 10.796 & 524.9 & 1.053 & & & & \\
\hline 394.0 & 10.919 & 524.8 & 1.058 & & & & \\
\hline 395.0 & 11.043 & 524.8 & 1.046 & & & & \\
\hline 396.0 & 11.168 & 524.8 & 1.046 & & & & \\
\hline 397.0 & 11.292 & 524.7 & 1.036 & & & & \\
\hline 355.0 & 6.015 & 475.9 & 1.159 & & & & \\
\hline 356.0 & 6.120 & 475.9 & 1.153 & & & & \\
\hline 357.0 & 6.225 & 475.9 & 1.163 & & & & \\
\hline 358.0 & 6.331 & 475.8 & 1.145 & & & & \\
\hline 359.0 & 6.438 & 475.8 & 1.105 & & & & \\
\hline 360.0 & 6.545 & 475.8 & 1.137 & & & & \\
\hline 361.0 & 6.651 & 475.8 & 1.128 & & & & \\
\hline 362.0 & 6.757 & 475.8 & 1.125 & & & & \\
\hline 363.0 & 6.864 & 475.8 & 1.122 & & & & \\
\hline 364.0 & 6.971 & 475.8 & 1.096 & & & & \\
\hline 365.0 & 7.077 & 475.8 & 1.102 & & & & \\
\hline 366.0 & 7.184 & 475.8 & 1.092 & & & & \\
\hline 367.0 & 7.291 & 475.8 & 1.091 & & & & \\
\hline 368.0 & 7.398 & 475.7 & 1.096 & & & & \\
\hline 369.0 & 7.505 & 475.7 & 1.094 & & & & \\
\hline 370.0 & 7.611 & 475.7 & 1.089 & & & & \\
\hline 371.0 & 7.718 & 475.7 & 1.075 & & & & \\
\hline 372.0 & 7.826 & 475.7 & 1.077 & & & & \\
\hline 373.0 & 7.933 & 475.7 & 1.073 & & & & \\
\hline 374.0 & 8.039 & 475.6 & 1.076 & & & & \\
\hline 375.0 & 8.146 & 475.6 & 1.085 & & & & \\
\hline 376.0 & 8.254 & 475.6 & 1.071 & & & & \\
\hline
\end{tabular}




\section{MIXTURE MODEL}

The mixture model developed here is based on corresponding states theory and uses reducing parameters which are dependent on the mole fraction of the mixture and critical point of the pure fluids to modify absolute values of density and temperature. This approach allows the thermodynamic values of the mixture to be based largely on the contributions from the pure fluids. Only the excess values, or the departure from ideal mixing, are required to accurately model the properties of the mixture.

In developing this model, an extensive collection of data, comprising more than 50,000 individual data points from more than 60 sources, was utilized. Data for the pressure-densitytemperature (P- $\rho-\mathrm{T})$ and vapor-liquid equilibria (VLE) behavior, speed of sound, heat capacity, and second virial coefficients for the blends R32/125, R32/134a, R125/134a, R125/143a, $\mathrm{R} 143 \mathrm{a} / 134 \mathrm{a}$, R134a/152a，R32/125/134a, and R125/143a/134a were included. A complete discussion of the mixture model, the available data, and comparisons of the data with the model are given in a paper to be submitted for publication in the Journal of Physical and Chemical Reference Data (Lemmon and Jacobsen 2002b). The manuscript is included here as Appendix B. A summary of the model follows.

The model uses the Helmholtz energy as the basis for all calculations. The Helmholtz energy of the mixture is calculated as the sum of an ideal gas contribution, a real gas contribution, and a contribution from mixing. The Helmholtz energy for an ideal solution (the contributions of the ideal gas and real gas) is determined at the reduced density and temperature of the mixture using accurate pure fluid equations of state for the mixture components. Reducing parameters, dependent on the mole fraction, are used to modify values of density and temperature.

The contribution from mixing, a modified excess function, is given by an empirical equation. Three separate models (i.e. three separate excess functions) were developed to calculate the properties of the refrigerant mixtures studied in this work. The first two describe the properties of the binary mixtures R32/125 and R32/134a. The shape of the excess functions for these two mixtures differ from each other and from the other mixtures studied in this work, and cannot be modeled in a generalized fashion. However, the shapes of the excess functions for the mixtures R125/134a, R125/143a, R134a/143a, and R134a/152a were similar enough that one function could be developed that described the properties of all these systems. Additionally, experimental data for the ternary mixtures R32/125/134a and R125/134a/143a showed that no additional parameters would be required to model these multi-component mixtures.

The equation for the mixture Helmholtz energy used in this work is

$$
a=a^{i d m i x}+a^{E} .
$$

where the Helmholtz energy for an ideal mixture is

$$
a^{i d m i x}=\sum_{i=1}^{n} x_{i}\left[a_{i}^{0}(\rho, T)+a_{i}^{r}(\delta, \tau)+R T \ln x_{i}\right]
$$

and where $\rho$ and $T$ are the mixture density and temperature, $\delta$ and $\tau$ are the reduced mixture density and temperature, $n$ is the number of components in the mixture, $a_{i}^{0}$ is the ideal gas Helmholtz energy of component $i, a_{i}^{\mathrm{r}}$ is the residual Helmholtz energy of component $i$, and the $x_{i}$ are the mole fractions of the mixture constituents. The Helmholtz energies of the components are given by the pure-fluid equations of state listed in Table 5. 
The reduced values of density and temperature used in Eq. (11) are

$$
\begin{aligned}
& \delta=\rho / \rho_{\text {red }} \text { and } \\
& \tau=T_{\text {red }} / T,
\end{aligned}
$$

where $\rho$ and $\mathrm{T}$ are the mixture density and temperature, and $\rho_{\text {red }}$ and $T_{\text {red }}$ are the reducing values,

$$
\begin{aligned}
& \rho_{\text {red }}=\left\lceil\sum_{i=1}^{n} \frac{x_{i}}{\rho_{c_{i}}}+\sum_{i=1}^{n-1} \sum_{j=i+1}^{n} x_{i} x_{j} \xi_{i j}\right\rceil^{-1} \text { and } \\
& T_{\text {red }}=\sum_{i=1}^{n} x_{i} T_{c_{i}}+\sum_{i=1}^{n-1} \sum_{j=i+1}^{n} x_{i} x_{j} \zeta_{i j} .
\end{aligned}
$$

The parameters $\zeta_{i j}$ and $\xi_{i j}$ are used to define the shapes of the reducing temperature and density curves, and they are determined simultaneously in the nonlinear fit of experimental data with the other parameters of the mixture model. These reducing parameters are not the same as the critical parameters of the mixture.

Three excess functions were developed for the mixtures studied in this work. The excess function for the mixture Helmholtz energy for these three models is expressed as

$$
\frac{a^{E}}{R T}=\alpha^{E}(\delta, \tau, \mathrm{x})=\left\{\sum_{i=1}^{n-1} \sum_{j=i+1}^{n} x_{i} x_{j} F_{i j}\right\} \sum_{k=1}^{m} N_{k} \delta^{d_{k}} \tau^{t_{k}} \exp \left(-\delta^{l_{k}}\right),
$$

where the coefficients and exponents were obtained from nonlinear regression of experimental mixture data. Values of the parameters and coefficients for these equations are given in Tables 6 and 7.

A complete discussion of the calculation of mixture properties from the above equations is given in Appendix B.

Based on comparisons to experimental data, the estimated uncertainties of properties calculated with the mixture model are $0.1 \%$ in density and $0.5 \%$ in heat capacities and the speed of sound. Calculated bubble point pressures are generally accurate to within $0.5 \%$. The model is valid from 200 to $450 \mathrm{~K}$ (-73 to $177^{\circ} \mathrm{C}$ ) with pressures up to $60 \mathrm{MPa}$. The equation was developed primarily using data for binary (two-component) blends, but it is accurate in calculating the properties of the two mixtures with three constituents for which data were available. It is expected that this result will apply to other ternary and higher-order systems as well. 
Table 5. Pure fluid equations of state for refrigerants used in the mixture model

\begin{tabular}{cccc}
\hline \hline Fluid & Author & Temperature & Maximum \\
Range (K) & Pressure (MPa) \\
\hline R32 & Tillner-Roth and Yokozeki (1997) & $136.34-435$ & 70 \\
R125 & Lemmon and Jacobsen (2002) & $172.52-500$ & 60 \\
R134a & Tillner-Roth and Baehr (1994) & $169.85-455$ & 70 \\
R143a & Lemmon and Jacobsen (2001) & $161.34-650$ & 100 \\
R152a & Outcalt and McLinden (1996) & $154.56-500$ & 60 \\
\hline
\end{tabular}

Table 6. Coefficients and exponents of the mixture equations

\begin{tabular}{|c|c|c|c|c|}
\hline$k$ & $N_{k}$ & $t_{k}$ & $d_{k}$ & $l_{k}$ \\
\hline \multicolumn{5}{|c|}{ R32/125 } \\
\hline 1 & -0.0072955 & 4.50 & 2 & 1 \\
\hline 2 & 0.078035 & 0.57 & 5 & 1 \\
\hline 3 & 0.61007 & 1.90 & 1 & 2 \\
\hline 4 & 0.64246 & 1.20 & 3 & 2 \\
\hline 5 & 0.014965 & 0.50 & 9 & 2 \\
\hline 6 & -0.34049 & 2.60 & 2 & 3 \\
\hline 7 & 0.085658 & 11.40 & 3 & 3 \\
\hline 8 & -0.064429 & 4.50 & 6 & 3 \\
\hline \multicolumn{5}{|c|}{ R32/134a } \\
\hline 1 & 0.22909 & 1.9 & 1 & 1 \\
\hline 2 & 0.094074 & 0.25 & 3 & 1 \\
\hline 3 & 0.00039876 & 0.07 & 8 & 1 \\
\hline 4 & 0.021133 & 2.0 & 1 & 2 \\
\hline \multicolumn{5}{|c|}{ R125/134a, R125/143a, R134a/143a, R143a/152a } \\
\hline 1 & -0.013073 & 7.4 & 1 & 1 \\
\hline 2 & 0.018259 & 0.35 & 3 & 1 \\
\hline 3 & 0.0000081299 & 10.0 & 11 & 2 \\
\hline 4 & 0.0078496 & 5.3 & 2 & 3 \\
\hline
\end{tabular}

Table 7. Parameters of the mixture equation

\begin{tabular}{lcll}
\hline \hline \multicolumn{1}{c}{ Binary Mixture } & $\zeta_{i j}(\mathrm{~K})$ & $\xi_{i j}(\mathrm{~L} / \mathrm{mol})$ & $F_{i j}$ \\
\hline $\mathrm{R} 32 / 125$ & 28.95 & -0.006008 & 1.0 \\
$\mathrm{R} 32 / 134 \mathrm{a}$ & 7.909 & -0.002039 & 1.0 \\
$\mathrm{R} 125 / 134 \mathrm{a}$ & -0.4326 & -0.0003453 & 1.0 \\
$\mathrm{R} 125 / 143 \mathrm{a}$ & 5.551 & -0.0004452 & 1.1697 \\
$\mathrm{R} 134 \mathrm{a} / 143 \mathrm{a}$ & 2.324 & 0.0006182 & 0.5557 \\
$\mathrm{R} 134 \mathrm{a} / 152 \mathrm{a}$ & 4.202 & 0.004223 & 2.0 \\
\hline
\end{tabular}




\section{MIXTURE THERMAL CONDUCTIVITY MEASUREMENTS}

Experimental. Cylinders of the R410A (R32/125) and R507A (R125/143a) mixtures were prepared gravimetrically from high-purity components. The R32 and R125 used in this work were "electronic grade" materials supplied by the Dupont Company. The manufacturer's specification stated a minimum purity of $99.99 \%$ with a water content less than 5 ppm. Our analysis by gas chromatography confirmed they were of very high purity apart from a small amout of air in the vapor phase. The R143a was refrigerant-grade material supplied by Honeywell; our analysis indicated a purity of $99.93 \%$. The air and any other light inorganics which may have been present in the components were removed by repeatedly freezing the sample in liquid nitrogen, evacuating the vapor space, and thawing. The target compositions for both blends were 0.50/0.50 mass fraction. The actual composition of the R507A (R125/143a) was $0.49983 / 0.50017$ mass fraction. The "second" cylinder of R410A was used for the thermal conductivity measurements; its composition was $0.49987 / 0.50013$ mass fraction. The uncertainty in compositions was \pm 0.00002 mass fraction.

A transient hot-wire instrument was used to make measurements of the thermal conductivity of both R410A and R507A. Two anodized tantalum hot wires, with diameters of $25 \mu \mathrm{m}$, were immersed in the sample. They function as both electrical heat sources and resistance thermometers during the experiments. The anodized layer of tantalum pentoxide provides electrical insulation from the refrigerant sample. The low-pressure gas mixtures were condensed (single phase) into the measurement cell at temperatures near $220 \mathrm{~K}$ and subsequently pressurized to near $35 \mathrm{MPa}$ by warming. The relative uncertainty, at $95 \%$ confidence, of the thermal conductivity measurements is less than $1 \%$ for most of the data, increasing to $3 \%$ in the critical region where the fluid is very compressible and prone to convection. Transient measurements were made over the entire range of conditions and were supplemented by steadystate measurements of the low-density vapor at pressures below $3 \mathrm{MPa}$. The transient hot-wire technique and apparatus are described in detail by Perkins et al. (1991). The use of the transient apparatus in the steady-state mode is described by Roder et al. (2000).

The thermal conductivities of R410A and R507A were each measured along six isotherms from 300 to $400 \mathrm{~K}$ with pressures to $35 \mathrm{MPa}$. Three of the isotherms for each blend are supercritical. Over 634 measurements were made for R410A, and more than 687 data points were measured for R507A. Figures 4 and 5 depict the measured points on temperature-pressure coordinates.

Results. The thermal conductivity data for R410A and R507A are summarized in Figures 6 and 7, respectively. The experimental data are presented in Tables $8-11$. These figures show that the thermal conductivity is a strong function of density and a somewhat weaker function of temperature. The critical enhancement is seen in the middle range of densities.

The present data are compared with the values calculated with the extended corresponding states model in REFPROP (Lemmon et al. 2002), which is the model of McLinden et al. (2000). This model is based only on the pure-fluid conductivities - there is no "mixture parameter" to account for interactions between dissimilar molecules. The need for such a mixture parameter is evident from examining the deviations between the present data and the model. The deviations for R410A (shown in Figure 8 and given in the data tables as $\Delta \lambda$ ) show a clear systematic deviation, which is especially pronounced at the higher densities. The largest deviations are seen for the isotherm at $360 \mathrm{~K}$ at densities near $500 \mathrm{~kg} / \mathrm{m}^{3}$. These conditions are near the critical point, suggesting that the critical enhancement in the mixture model needs improvement. Systematic deviations are also seen for R507A (Figure 9), although here the model and data agree fairly well at high densities but show increasing deviations at the lower densities. There is little temperature dependence and deviations near the critical point are no higher than away from it. 


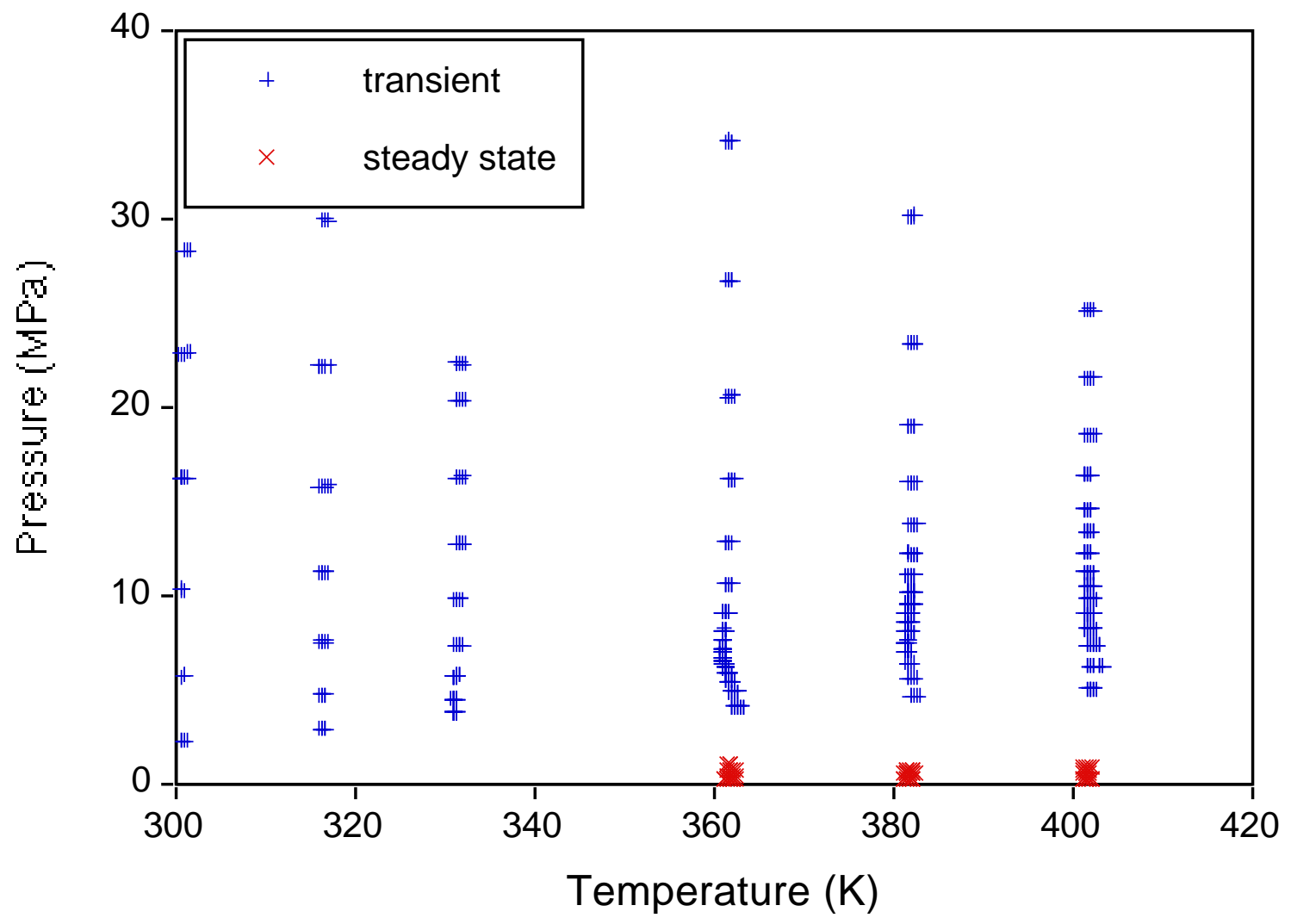

Figure 4. Location of measured thermal conductivity data points for R410A 


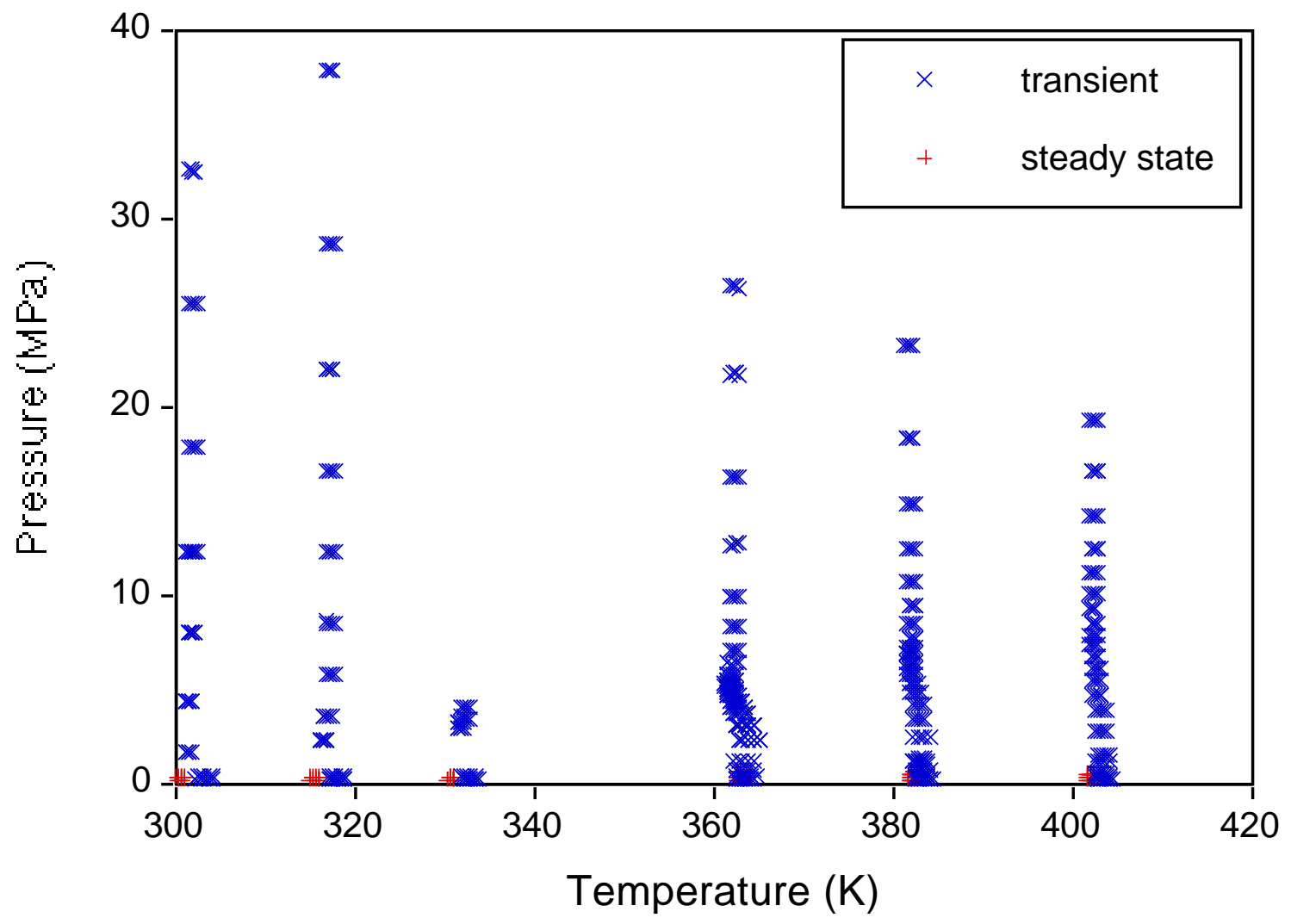

Figure 5. Location of measured thermal conductivity data points for R507A. 


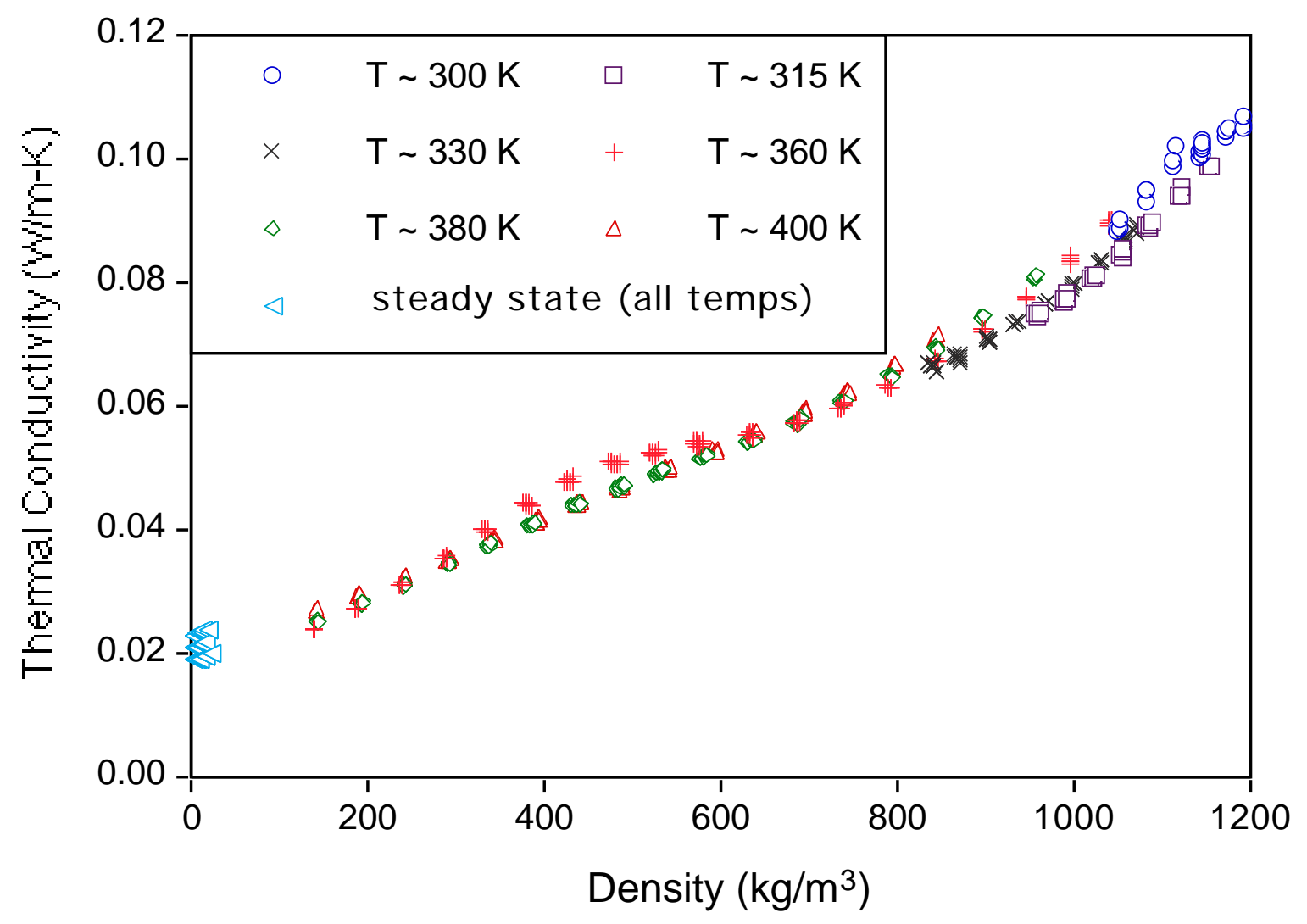

Figure 6. Thermal conductivity of R410A. 


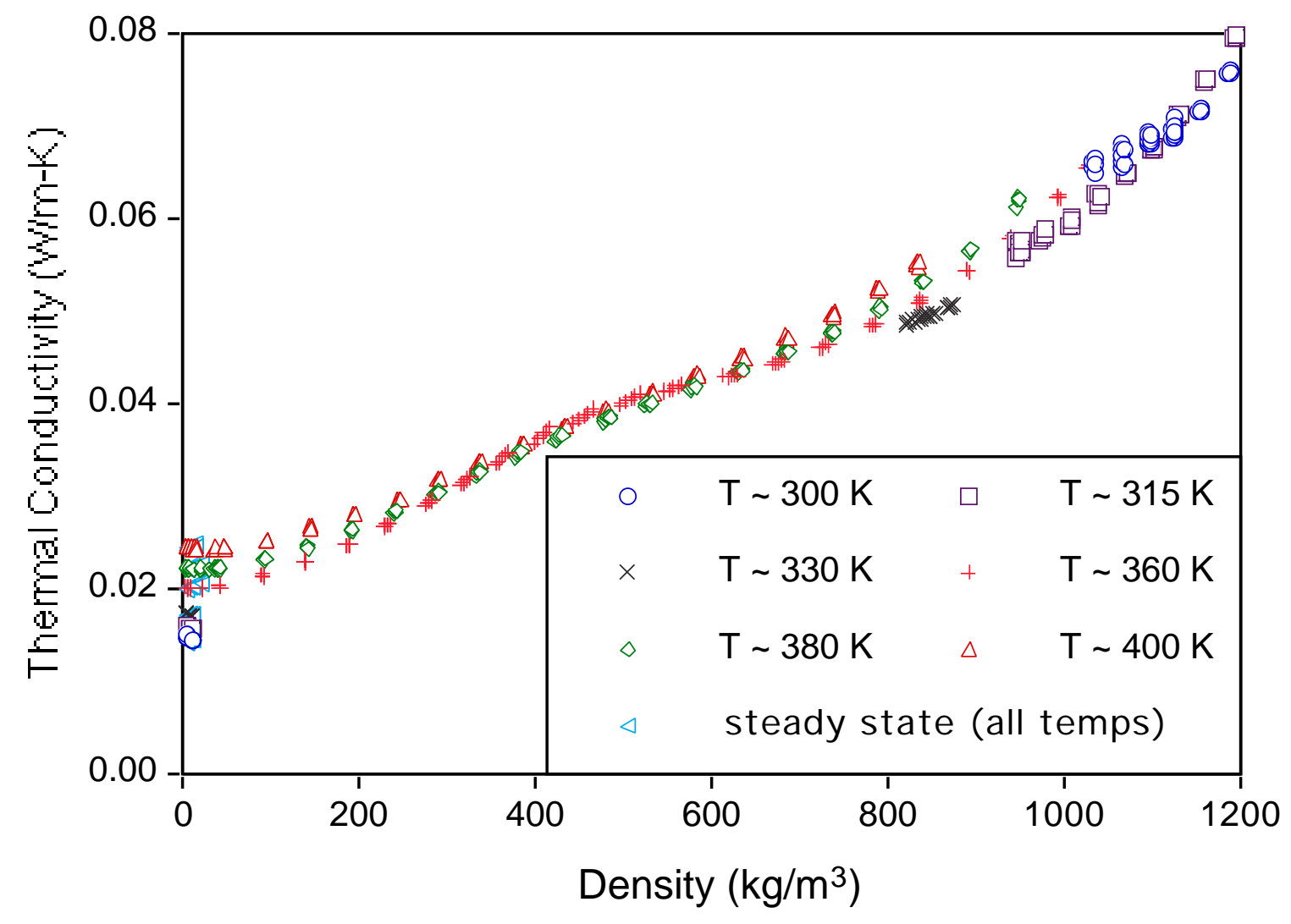

Figure 7. Thermal conductivity of R507A. 


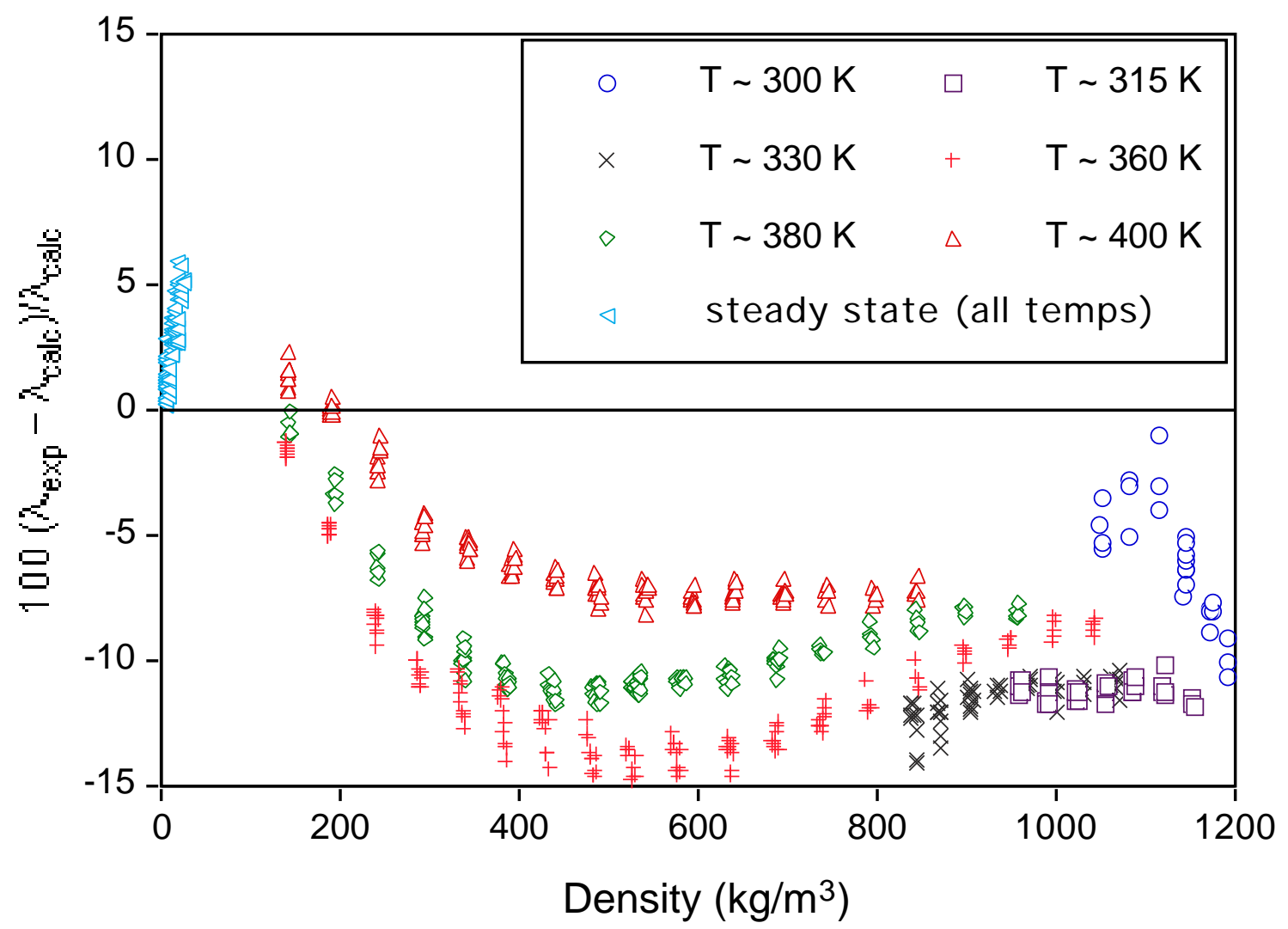

Figure 8. Deviations between the measured thermal conductivities for R410A and the model in REFPROP. 


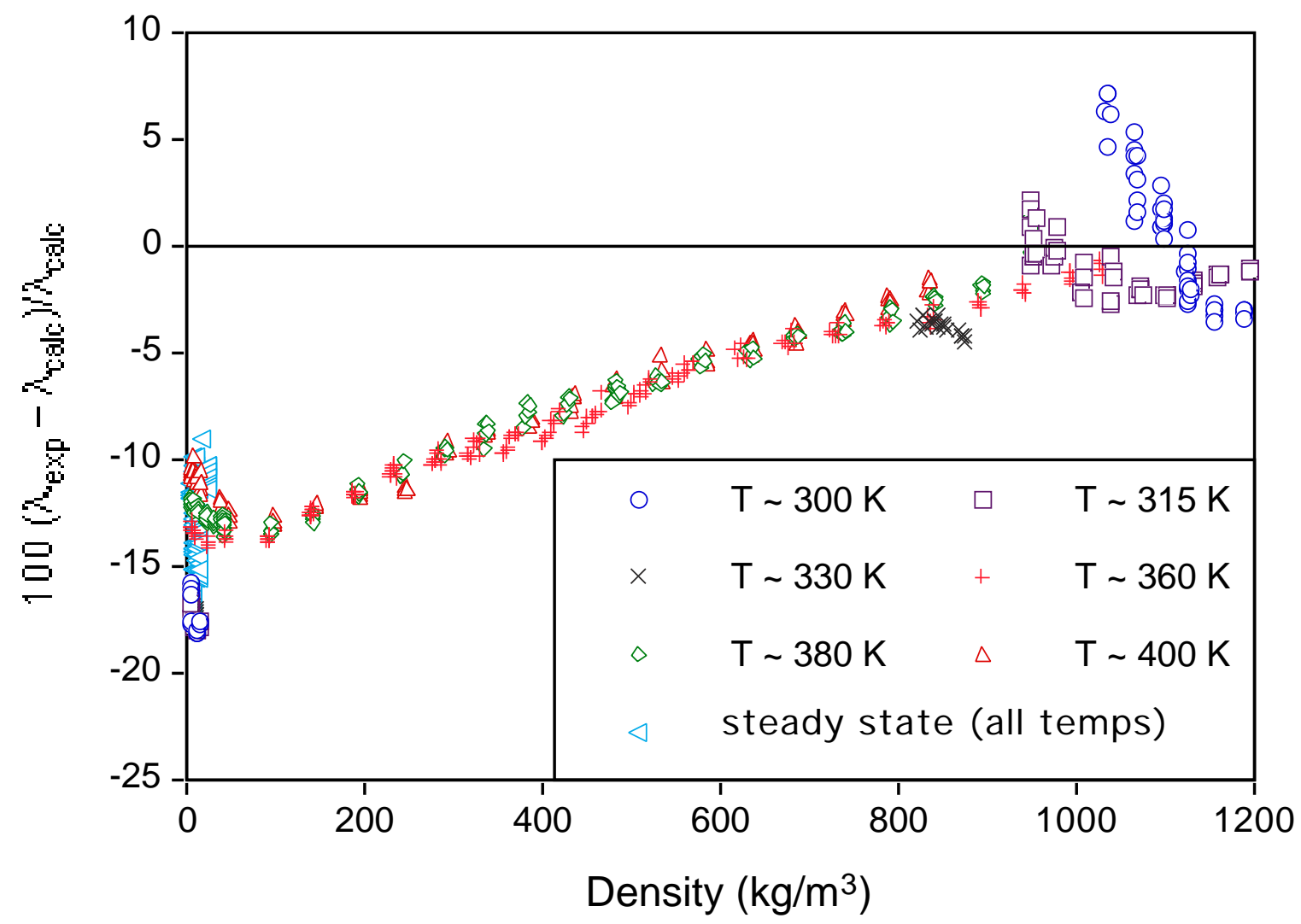

Figure 9. Deviations between the measured thermal conductivities for R507A and the model in REFPROP. 
Table 8. Thermal conductivity for R410A measured by the steady-state technique. The column $\lambda_{\text {calc }}$ gives values calculated by the NIST REFPROP database, and $\Delta \lambda$ is the difference between the experimental data and these calculated values.

\begin{tabular}{|c|c|c|c|c|c|}
\hline $\begin{array}{c}\text { Temperature } \\
\text { (K) }\end{array}$ & $\begin{array}{c}\text { Pressure } \\
(\mathrm{MPa})\end{array}$ & $\begin{array}{c}\text { Density (calc) } \\
\left(\mathrm{kg} / \mathrm{m}^{3}\right)\end{array}$ & $\begin{array}{c}\lambda_{\exp } \\
(\mathrm{W} / \mathrm{m} \cdot \mathrm{K})\end{array}$ & $\begin{array}{c}\lambda_{\text {calc }} \\
(\mathrm{W} / \mathrm{m} \cdot \mathrm{K})\end{array}$ & $\begin{array}{l}\Delta \lambda \\
(\%)\end{array}$ \\
\hline 361.489 & 0.902 & 23.17 & 0.01962 & 0.01867 & 5.09 \\
\hline 361.671 & 0.903 & 23.17 & 0.01963 & 0.01869 & 5.05 \\
\hline 361.894 & 0.903 & 23.15 & 0.01966 & 0.01871 & 5.10 \\
\hline 361.526 & 0.626 & 15.76 & 0.01932 & 0.01866 & 3.53 \\
\hline 361.716 & 0.626 & 15.74 & 0.01928 & 0.01868 & 3.22 \\
\hline 361.962 & 0.625 & 15.71 & 0.01921 & 0.01870 & 2.72 \\
\hline 362.201 & 0.625 & 15.70 & 0.01921 & 0.01872 & 2.61 \\
\hline 362.443 & 0.626 & 15.70 & 0.01926 & 0.01874 & 2.75 \\
\hline 361.577 & 0.319 & 7.87 & 0.01900 & 0.01866 & 1.83 \\
\hline 361.770 & 0.320 & 7.88 & 0.01897 & 0.01868 & 1.57 \\
\hline 362.236 & 0.319 & 7.85 & 0.01893 & 0.01872 & 1.13 \\
\hline 362.526 & 0.320 & 7.86 & 0.01897 & 0.01874 & 1.20 \\
\hline 361.790 & 0.206 & 5.03 & 0.01886 & 0.01868 & 0.98 \\
\hline 362.023 & 0.206 & 5.03 & 0.01883 & 0.01870 & 0.71 \\
\hline 362.274 & 0.207 & 5.05 & 0.01886 & 0.01872 & 0.75 \\
\hline 362.531 & 0.206 & 5.04 & 0.01884 & 0.01874 & 0.51 \\
\hline 361.304 & 0.208 & 5.09 & 0.01885 & 0.01863 & 1.17 \\
\hline 361.515 & 0.209 & 5.10 & 0.01888 & 0.01865 & 1.22 \\
\hline 361.744 & 0.209 & 5.11 & 0.01890 & 0.01867 & 1.22 \\
\hline 362.006 & 0.208 & 5.08 & 0.01884 & 0.01870 & 0.77 \\
\hline 362.260 & 0.208 & 5.08 & 0.01886 & 0.01872 & 0.75 \\
\hline 361.325 & 0.108 & 2.63 & 0.01882 & 0.01863 & 1.00 \\
\hline 361.522 & 0.108 & 2.61 & 0.01874 & 0.01865 & 0.47 \\
\hline 361.765 & 0.108 & 2.62 & 0.01874 & 0.01867 & 0.35 \\
\hline 362.010 & 0.108 & 2.63 & 0.01876 & 0.01870 & 0.34 \\
\hline 381.340 & 0.710 & 16.90 & 0.02171 & 0.02051 & 5.87 \\
\hline 381.574 & 0.710 & 16.89 & 0.02158 & 0.02053 & 5.12 \\
\hline 381.792 & 0.710 & 16.86 & 0.02157 & 0.02055 & 4.97 \\
\hline 382.052 & 0.709 & 16.84 & 0.02152 & 0.02057 & 4.60 \\
\hline 382.304 & 0.710 & 16.85 & 0.02150 & 0.02060 & 4.38 \\
\hline 381.391 & 0.540 & 12.72 & 0.02122 & 0.02051 & 3.48 \\
\hline 381.636 & 0.540 & 12.72 & 0.02118 & 0.02053 & 3.16 \\
\hline 381.885 & 0.540 & 12.71 & 0.02117 & 0.02055 & 3.00 \\
\hline 382.146 & 0.541 & 12.71 & 0.02114 & 0.02058 & 2.72 \\
\hline 382.387 & 0.540 & 12.70 & 0.02114 & 0.02060 & 2.61 \\
\hline 381.266 & 0.416 & 9.75 & 0.02113 & 0.02049 & 3.11 \\
\hline 381.494 & 0.416 & 9.72 & 0.02104 & 0.02051 & 2.56 \\
\hline 381.736 & 0.415 & 9.71 & 0.02101 & 0.02054 & 2.30 \\
\hline 381.974 & 0.416 & 9.72 & 0.02100 & 0.02056 & 2.14 \\
\hline 382.240 & 0.417 & 9.73 & 0.02102 & 0.02059 & 2.11 \\
\hline 381.525 & 0.211 & 4.89 & 0.02078 & 0.02052 & 1.29 \\
\hline 381.768 & 0.212 & 4.89 & 0.02078 & 0.02054 & 1.17 \\
\hline 382.013 & 0.211 & 4.87 & 0.02080 & 0.02056 & 1.15 \\
\hline 382.280 & 0.210 & 4.86 & 0.02077 & 0.02059 & \\
\hline 381.127 & 0.109 & 2.52 & 0.02064 & 0.02048 & 0.8 \\
\hline
\end{tabular}


Table 8. Thermal conductivity for R410A (continued)

$\begin{array}{crcccc}\begin{array}{c}\text { Temperature } \\ (\mathrm{K})\end{array} & \begin{array}{c}\text { Pressure } \\ (\mathrm{MPa})\end{array} & \begin{array}{c}\text { Density }(\text { calc }) \\ \left(\mathrm{kg} / \mathrm{m}^{3}\right)\end{array} & \begin{array}{c}\lambda_{\text {exp }} \\ (\mathrm{W} / \mathrm{m} \cdot \mathrm{K})\end{array} & \begin{array}{c}\lambda \text { calc } \\ (\mathrm{W} / \mathrm{m} \cdot \mathrm{K})\end{array} & \begin{array}{c}\Delta \lambda \\ (\%)\end{array} \\ 381.333 & 0.109 & 2.50 & 0.02059 & 0.02050 & 0.46 \\ 381.550 & 0.108 & 2.49 & 0.02057 & 0.02052 & 0.26 \\ 381.773 & 0.109 & 2.50 & 0.02057 & 0.02054 & 0.15 \\ 382.023 & 0.109 & 2.51 & 0.02059 & 0.02056 & 0.13 \\ & & & & & \\ 401.168 & 0.838 & 18.94 & 0.02375 & 0.02247 & 5.71 \\ 401.352 & 0.838 & 18.92 & 0.02358 & 0.02249 & 4.87 \\ 401.745 & 0.838 & 18.91 & 0.02355 & 0.02253 & 4.55 \\ 402.009 & 0.838 & 18.89 & 0.02353 & 0.02255 & 4.33 \\ 401.174 & 0.625 & 13.98 & 0.02351 & 0.02246 & 4.66 \\ 401.582 & 0.624 & 13.95 & 0.02337 & 0.02251 & 3.84 \\ 401.812 & 0.625 & 13.95 & 0.02343 & 0.02253 & 4.00 \\ 401.999 & 0.624 & 13.93 & 0.02336 & 0.02255 & 3.60 \\ 401.185 & 0.415 & 9.20 & 0.02328 & 0.02246 & 3.64 \\ 401.385 & 0.416 & 9.21 & 0.02326 & 0.02248 & 3.46 \\ 401.576 & 0.416 & 9.21 & 0.02318 & 0.02250 & 3.01 \\ 401.794 & 0.415 & 9.19 & 0.02314 & 0.02252 & 2.73 \\ 401.225 & 0.211 & 4.63 & 0.02309 & 0.02246 & 2.78 \\ 401.428 & 0.210 & 4.61 & 0.02292 & 0.02249 & 1.93 \\ 401.638 & 0.211 & 4.62 & 0.02295 & 0.02251 & 1.97 \\ 401.842 & 0.211 & 4.63 & 0.02300 & 0.02253 & 2.10 \\ 402.077 & 0.210 & 4.60 & 0.02296 & 0.02255 & 1.81 \\ 401.230 & 0.109 & 2.37 & 0.02278 & 0.02246 & 1.40 \\ 401.402 & 0.110 & 2.39 & 0.02268 & 0.02248 & 0.88 \\ 401.615 & 0.109 & 2.38 & 0.02274 & 0.02250 & 1.05 \\ 401.850 & 0.108 & 2.37 & 0.02270 & 0.02253 & 0.76 \\ 402.082 & 0.109 & 2.38 & 0.02274 & 0.02255 & 0.84\end{array}$


Table 9. Thermal conductivity for R410A measured by the transient hot-wire technique; data are listed in the order of measurement.

\begin{tabular}{|c|c|c|c|c|c|}
\hline $\begin{array}{c}\text { Temperature } \\
\text { (K) }\end{array}$ & $\begin{array}{c}\text { Pressure } \\
(\mathrm{MPa})\end{array}$ & $\begin{array}{c}\text { Density (calc) } \\
\left(\mathrm{kg} / \mathrm{m}^{3}\right)\end{array}$ & $\begin{array}{c}\lambda_{\exp } \\
(\mathrm{W} / \mathrm{m} \cdot \mathrm{K})\end{array}$ & $\begin{array}{c}\lambda_{\text {calc }} \\
(\mathrm{W} / \mathrm{m} \cdot \mathrm{K})\end{array}$ & $\begin{array}{l}\Delta \lambda \\
(\%)\end{array}$ \\
\hline 300.976 & 28.25 & 1191.0 & 0.10684 & 0.11756 & -9.12 \\
\hline 301.258 & 28.26 & 1190.2 & 0.10480 & 0.11744 & -10.76 \\
\hline 301.571 & 28.24 & 1189.3 & 0.10546 & 0.11728 & -10.08 \\
\hline 300.472 & 22.79 & 1172.7 & 0.10503 & 0.11376 & -7.67 \\
\hline 300.740 & 22.78 & 1171.8 & 0.10448 & 0.11362 & -8.05 \\
\hline 301.034 & 22.79 & 1170.9 & 0.10428 & 0.11349 & -8.11 \\
\hline 301.328 & 22.83 & 1170.2 & 0.10321 & 0.11337 & -8.97 \\
\hline 301.645 & 22.84 & 1169.3 & 0.10427 & 0.11324 & -7.92 \\
\hline 300.513 & 16.17 & 1144.3 & 0.10211 & 0.10836 & -5.77 \\
\hline 300.767 & 16.15 & 1143.3 & 0.10167 & 0.10821 & -6.05 \\
\hline 301.053 & 16.13 & 1142.3 & 0.10115 & 0.10806 & -6.39 \\
\hline 301.373 & 16.13 & 1141.2 & 0.09986 & 0.10789 & -7.44 \\
\hline 300.552 & 16.20 & 1144.3 & 0.10260 & 0.10836 & -5.32 \\
\hline 300.660 & 16.19 & 1143.9 & 0.10274 & 0.10830 & -5.14 \\
\hline 300.824 & 16.16 & 1143.2 & 0.10125 & 0.10820 & -6.42 \\
\hline 300.977 & 16.15 & 1142.6 & 0.10055 & 0.10811 & -6.99 \\
\hline 300.591 & 10.26 & 1112.8 & 0.10182 & 0.10288 & -1.03 \\
\hline 300.724 & 10.25 & 1112.2 & 0.09965 & 0.10280 & -3.07 \\
\hline 300.868 & 10.25 & 1111.7 & 0.09856 & 0.10272 & -4.05 \\
\hline 300.766 & 5.64 & 1080.7 & 0.09488 & 0.09787 & -3.06 \\
\hline 300.920 & 5.66 & 1080.2 & 0.09499 & 0.09780 & -2.87 \\
\hline 301.072 & 5.67 & 1079.5 & 0.09270 & 0.09772 & -5.14 \\
\hline 300.687 & 2.18 & 1050.1 & 0.09020 & 0.09357 & -3.61 \\
\hline 300.820 & 2.19 & 1049.4 & 0.08854 & 0.09350 & -5.30 \\
\hline 300.969 & 2.19 & 1048.7 & 0.08824 & 0.09341 & -5.53 \\
\hline 301.292 & 2.17 & 1046.6 & 0.08880 & 0.09317 & -4.69 \\
\hline 361.382 & 34.06 & 1041.7 & 0.08996 & 0.09814 & -8.33 \\
\hline 361.544 & 34.07 & 1041.3 & 0.08967 & 0.09809 & -8.58 \\
\hline 361.708 & 34.06 & 1040.7 & 0.08963 & 0.09802 & -8.56 \\
\hline 361.893 & 34.04 & 1040.1 & 0.08898 & 0.09794 & -9.15 \\
\hline 362.073 & 34.03 & 1039.5 & 0.08924 & 0.09788 & -8.83 \\
\hline 361.408 & 26.64 & 997.0 & 0.08383 & 0.09161 & -8.49 \\
\hline 361.572 & 26.66 & 996.6 & 0.08396 & 0.09156 & -8.30 \\
\hline 361.750 & 26.66 & 996.0 & 0.08315 & 0.09151 & -9.13 \\
\hline 361.932 & 26.65 & 995.3 & 0.08334 & 0.09143 & -8.85 \\
\hline 362.139 & 26.64 & 994.6 & 0.08285 & 0.09134 & -9.30 \\
\hline 361.434 & 20.48 & 947.4 & 0.07755 & 0.08533 & -9.12 \\
\hline 361.624 & 20.48 & 946.7 & 0.07745 & 0.08525 & -9.15 \\
\hline 361.821 & 20.49 & 946.0 & 0.07701 & 0.08519 & -9.61 \\
\hline 362.022 & 20.50 & 945.4 & 0.07704 & 0.08513 & -9.50 \\
\hline 362.221 & 20.51 & 944.6 & 0.07719 & 0.08505 & -9.25 \\
\hline 361.542 & 16.07 & 898.0 & 0.07212 & 0.08001 & -9.86 \\
\hline 361.730 & 16.07 & 897.1 & 0.07215 & 0.07992 & -9.73 \\
\hline 361.934 & 16.07 & 896.2 & 0.07170 & 0.07984 & -10.20 \\
\hline 362.152 & 16.08 & 895.3 & 0.07208 & 0.07976 & -9.63 \\
\hline 362.371 & 16.09 & 894.3 & 0.07211 & 0.07967 & -9.49 \\
\hline
\end{tabular}


Table 9. Thermal conductivity for R410A (continued)

\begin{tabular}{|c|c|c|c|c|c|}
\hline $\begin{array}{c}\text { Temperature } \\
\text { (K) }\end{array}$ & $\begin{array}{c}\text { Pressure } \\
(\mathrm{MPa})\end{array}$ & $\begin{array}{c}\text { Density (calc) } \\
\left(\mathrm{kg} / \mathrm{m}^{3}\right)\end{array}$ & $\begin{array}{c}\lambda_{\exp } \\
(\mathrm{W} / \mathrm{m} \cdot \mathrm{K})\end{array}$ & $\begin{array}{c}\lambda_{\text {calc }} \\
(\mathrm{W} / \mathrm{m} \cdot \mathrm{K})\end{array}$ & $\begin{array}{l}\Delta \lambda \\
(\%)\end{array}$ \\
\hline 361.241 & 12.73 & 846.3 & 0.06691 & 0.07533 & -11.18 \\
\hline 361.402 & 12.72 & 845.3 & 0.06675 & 0.07525 & -11.29 \\
\hline 361.605 & 12.72 & 844.0 & 0.06703 & 0.07514 & -10.80 \\
\hline 361.803 & 12.72 & 842.8 & 0.06701 & 0.07505 & -10.71 \\
\hline 362.009 & 12.73 & 841.6 & 0.06744 & 0.07496 & -10.03 \\
\hline 361.278 & 10.49 & 792.4 & 0.06282 & 0.07134 & -11.94 \\
\hline 361.464 & 10.50 & 791.0 & 0.06275 & 0.07124 & -11.92 \\
\hline 361.654 & 10.50 & 789.6 & 0.06269 & 0.07114 & -11.88 \\
\hline 361.863 & 10.49 & 787.9 & 0.06244 & 0.07102 & -12.08 \\
\hline 362.092 & 10.49 & 786.0 & 0.06319 & 0.07089 & -10.86 \\
\hline 360.975 & 9.01 & 740.2 & 0.06031 & 0.06823 & -11.61 \\
\hline 360.981 & 9.01 & 740.1 & 0.05994 & 0.06823 & -12.15 \\
\hline 361.145 & 9.02 & 738.5 & 0.06001 & 0.06813 & -11.92 \\
\hline 361.139 & 9.02 & 738.6 & 0.05974 & 0.06814 & -12.33 \\
\hline 361.309 & 9.02 & 736.9 & 0.05938 & 0.06804 & -12.72 \\
\hline 361.310 & 9.02 & 737.0 & 0.05924 & 0.06804 & -12.93 \\
\hline 361.493 & 9.02 & 735.1 & 0.05936 & 0.06793 & -12.61 \\
\hline 361.499 & 9.02 & 735.0 & 0.05947 & 0.06792 & -12.45 \\
\hline 361.703 & 9.02 & 732.9 & 0.05925 & 0.06780 & -12.61 \\
\hline 361.701 & 9.02 & 733.0 & 0.05921 & 0.06780 & -12.67 \\
\hline 360.844 & 8.10 & 689.0 & 0.05690 & 0.06587 & -13.61 \\
\hline 360.951 & 8.10 & 687.6 & 0.05743 & 0.06580 & -12.71 \\
\hline 360.957 & 8.10 & 687.5 & 0.05756 & 0.06579 & -12.51 \\
\hline 361.088 & 8.10 & 685.8 & 0.05723 & 0.06570 & -12.90 \\
\hline 361.093 & 8.11 & 685.7 & 0.05672 & 0.06570 & -13.67 \\
\hline 361.221 & 8.10 & 683.9 & 0.05676 & 0.06561 & -13.49 \\
\hline 361.227 & 8.10 & 683.7 & 0.05690 & 0.06560 & -13.27 \\
\hline 361.375 & 8.10 & 681.7 & 0.05673 & 0.06550 & -13.39 \\
\hline 361.381 & 8.10 & 681.6 & 0.05681 & 0.06549 & -13.26 \\
\hline 360.880 & 7.52 & 637.0 & 0.05524 & 0.06408 & -13.79 \\
\hline 360.874 & 7.52 & 637.1 & 0.05547 & 0.06408 & -13.44 \\
\hline 360.959 & 7.52 & 635.5 & 0.05463 & 0.06402 & -14.66 \\
\hline 360.968 & 7.53 & 635.4 & 0.05475 & 0.06401 & -14.47 \\
\hline 361.041 & 7.53 & 634.2 & 0.05534 & 0.06396 & -13.48 \\
\hline 361.050 & 7.53 & 634.1 & 0.05527 & 0.06395 & -13.58 \\
\hline 361.133 & 7.53 & 632.7 & 0.05540 & 0.06389 & -13.29 \\
\hline 361.140 & 7.53 & 632.6 & 0.05550 & 0.06389 & -13.13 \\
\hline 361.232 & 7.53 & 630.9 & 0.05516 & 0.06382 & -13.56 \\
\hline 361.236 & 7.53 & 630.7 & 0.05509 & 0.06381 & -13.66 \\
\hline 360.831 & 7.09 & 578.8 & 0.05352 & 0.06256 & -14.46 \\
\hline 360.831 & 7.10 & 578.9 & 0.05405 & 0.06257 & -13.61 \\
\hline 360.923 & 7.09 & 576.6 & 0.05331 & 0.06247 & -14.66 \\
\hline 360.925 & 7.09 & 576.5 & 0.05351 & 0.06247 & -14.34 \\
\hline 361.028 & 7.09 & 574.0 & 0.05333 & 0.06236 & -14.48 \\
\hline 361.020 & 7.09 & 574.2 & 0.05379 & 0.06237 & -13.76 \\
\hline 361.117 & 7.09 & 571.9 & 0.05395 & 0.06227 & -13.36 \\
\hline 361.101 & 7.09 & 572.3 & 0.05396 & 0.06229 & -13.37 \\
\hline
\end{tabular}


Table 9. Thermal conductivity for R410A (continued)

\begin{tabular}{|c|c|c|c|c|c|}
\hline $\begin{array}{c}\text { Temperature } \\
\text { (K) }\end{array}$ & $\begin{array}{c}\text { Pressure } \\
(\mathrm{MPa})\end{array}$ & $\begin{array}{c}\text { Density (calc) } \\
\left(\mathrm{kg} / \mathrm{m}^{3}\right)\end{array}$ & $\begin{array}{c}\lambda_{\exp } \\
(\mathrm{W} / \mathrm{m} \cdot \mathrm{K})\end{array}$ & $\begin{array}{c}\lambda_{\text {calc }} \\
(\mathrm{W} / \mathrm{m} \cdot \mathrm{K})\end{array}$ & $\begin{array}{l}\Delta \lambda \\
(\%)\end{array}$ \\
\hline 361.204 & 7.10 & 569.9 & 0.05374 & 0.06218 & -13.57 \\
\hline 361.208 & 7.10 & 569.9 & 0.05414 & 0.06218 & -12.93 \\
\hline 360.818 & 6.84 & 529.0 & 0.05256 & 0.06103 & -13.87 \\
\hline 360.824 & 6.84 & 528.7 & 0.05207 & 0.06101 & -14.66 \\
\hline 360.930 & 6.84 & 525.7 & 0.05193 & 0.06085 & -14.65 \\
\hline 360.921 & 6.84 & 526.0 & 0.05212 & 0.06086 & -14.36 \\
\hline 361.010 & 6.84 & 523.5 & 0.05169 & 0.06072 & -14.87 \\
\hline 361.008 & 6.84 & 523.6 & 0.05161 & 0.06072 & -15.01 \\
\hline 361.089 & 6.84 & 521.5 & 0.05237 & 0.06060 & -13.58 \\
\hline 361.108 & 6.84 & 521.1 & 0.05233 & 0.06057 & -13.61 \\
\hline 361.202 & 6.84 & 518.5 & 0.05229 & 0.06042 & -13.46 \\
\hline 361.203 & 6.84 & 518.6 & 0.05208 & 0.06042 & -13.81 \\
\hline 360.819 & 6.66 & 485.0 & 0.05077 & 0.05893 & -13.85 \\
\hline 360.838 & 6.66 & 484.5 & 0.05035 & 0.05889 & -14.50 \\
\hline 360.924 & 6.66 & 482.0 & 0.05009 & 0.05870 & -14.67 \\
\hline 360.937 & 6.66 & 481.6 & 0.05010 & 0.05867 & -14.60 \\
\hline 361.028 & 6.66 & 479.1 & 0.05031 & 0.05847 & -13.96 \\
\hline 361.037 & 6.66 & 478.9 & 0.05031 & 0.05845 & -13.92 \\
\hline 361.125 & 6.66 & 476.5 & 0.05057 & 0.05826 & -13.20 \\
\hline 361.110 & 6.67 & 477.0 & 0.05029 & 0.05830 & -13.73 \\
\hline 361.222 & 6.67 & 474.0 & 0.05052 & 0.05805 & -12.97 \\
\hline 361.219 & 6.67 & 474.2 & 0.05087 & 0.05806 & -12.39 \\
\hline 360.863 & 6.47 & 432.3 & 0.04722 & 0.05513 & -14.34 \\
\hline 360.871 & 6.47 & 432.1 & 0.04828 & 0.05511 & -12.39 \\
\hline 360.966 & 6.47 & 429.7 & 0.04730 & 0.05486 & -13.77 \\
\hline 360.982 & 6.47 & 429.3 & 0.04728 & 0.05481 & -13.75 \\
\hline 361.080 & 6.47 & 426.9 & 0.04761 & 0.05456 & -12.74 \\
\hline 361.094 & 6.47 & 426.6 & 0.04794 & 0.05453 & -12.08 \\
\hline 361.196 & 6.47 & 424.3 & 0.04770 & 0.05427 & -12.11 \\
\hline 361.168 & 6.47 & 425.0 & 0.04754 & 0.05435 & -12.53 \\
\hline 361.291 & 6.47 & 422.2 & 0.04750 & 0.05405 & -12.11 \\
\hline 361.283 & 6.47 & 422.5 & 0.04738 & 0.05408 & -12.38 \\
\hline 360.951 & 6.29 & 384.9 & 0.04348 & 0.05060 & -14.06 \\
\hline 360.955 & 6.29 & 384.9 & 0.04373 & 0.05059 & -13.56 \\
\hline 361.050 & 6.29 & 383.0 & 0.04364 & 0.05036 & -13.34 \\
\hline 361.075 & 6.29 & 382.5 & 0.04397 & 0.05029 & -12.58 \\
\hline 361.169 & 6.29 & 380.8 & 0.04401 & 0.05008 & -12.11 \\
\hline 361.175 & 6.29 & 380.7 & 0.04359 & 0.05006 & -12.93 \\
\hline 361.303 & 6.29 & 378.4 & 0.04399 & 0.04978 & -11.63 \\
\hline 361.300 & 6.29 & 378.6 & 0.04423 & 0.04979 & -11.17 \\
\hline 361.430 & 6.29 & 376.3 & 0.04392 & 0.04951 & -11.28 \\
\hline 361.415 & 6.29 & 376.6 & 0.04385 & 0.04955 & -11.50 \\
\hline 361.096 & 6.07 & 337.1 & 0.03949 & 0.04527 & -12.77 \\
\hline 361.104 & 6.07 & 337.1 & 0.03967 & 0.04527 & -12.37 \\
\hline 361.225 & 6.07 & 335.6 & 0.03961 & 0.04506 & -12.09 \\
\hline 361.235 & 6.07 & 335.5 & 0.03955 & 0.04505 & -12.20 \\
\hline 361.352 & 6.07 & 334.1 & 0.03991 & 0.04485 & -11.01 \\
\hline
\end{tabular}


Table 9. Thermal conductivity for R410A (continued)

\begin{tabular}{|c|c|c|c|c|c|}
\hline $\begin{array}{c}\text { Temperature } \\
\text { (K) }\end{array}$ & $\begin{array}{c}\text { Pressure } \\
(\mathrm{MPa})\end{array}$ & $\begin{array}{c}\text { Density (calc) } \\
\left(\mathrm{kg} / \mathrm{m}^{3}\right)\end{array}$ & $\begin{array}{c}\lambda_{\exp } \\
(\mathrm{W} / \mathrm{m} \cdot \mathrm{K})\end{array}$ & $\begin{array}{c}\lambda_{\text {calc }} \\
(\mathrm{W} / \mathrm{m} \cdot \mathrm{K})\end{array}$ & $\begin{array}{l}\Delta \lambda \\
(\%)\end{array}$ \\
\hline 361.362 & 6.07 & 334.0 & 0.03994 & 0.04484 & -10.92 \\
\hline 361.487 & 6.07 & 332.5 & 0.03958 & 0.04048 & -2.23 \\
\hline 361.487 & 6.07 & 332.5 & 0.03958 & 0.04463 & -11.31 \\
\hline 361.496 & 6.07 & 332.3 & 0.03936 & 0.04461 & -11.77 \\
\hline 361.639 & 6.07 & 330.5 & 0.03967 & 0.04436 & -10.58 \\
\hline 361.641 & 6.07 & 330.4 & 0.03975 & 0.04435 & -10.38 \\
\hline 361.290 & 5.79 & 289.7 & 0.03526 & 0.03966 & -11.09 \\
\hline 361.283 & 5.79 & 289.8 & 0.03548 & 0.03966 & -10.55 \\
\hline 361.440 & 5.79 & 288.4 & 0.03520 & 0.03947 & -10.82 \\
\hline 361.447 & 5.79 & 288.3 & 0.03508 & 0.03946 & -11.09 \\
\hline 361.602 & 5.79 & 287.0 & 0.03492 & 0.03928 & -11.10 \\
\hline 361.598 & 5.79 & 287.0 & 0.03498 & 0.03928 & -10.95 \\
\hline 361.763 & 5.79 & 285.7 & 0.03495 & 0.03910 & -10.61 \\
\hline 361.763 & 5.79 & 285.8 & 0.03504 & 0.03910 & -10.39 \\
\hline 361.938 & 5.79 & 284.5 & 0.03503 & 0.03892 & -10.00 \\
\hline 361.932 & 5.79 & 284.6 & 0.03504 & 0.03893 & -10.00 \\
\hline 361.448 & 5.39 & 240.2 & 0.03101 & 0.03387 & -8.43 \\
\hline 361.457 & 5.39 & 240.2 & 0.03108 & 0.03386 & -8.22 \\
\hline 361.613 & 5.39 & 239.5 & 0.03057 & 0.03377 & -9.48 \\
\hline 361.624 & 5.39 & 239.5 & 0.03075 & 0.03377 & -8.94 \\
\hline 361.796 & 5.39 & 238.7 & 0.03087 & 0.03367 & -8.32 \\
\hline 361.795 & 5.39 & 238.7 & 0.03071 & 0.03367 & -8.80 \\
\hline 361.978 & 5.39 & 237.9 & 0.03082 & 0.03357 & -8.18 \\
\hline 361.989 & 5.39 & 237.9 & 0.03066 & 0.03356 & -8.65 \\
\hline 362.187 & 5.39 & 237.0 & 0.03075 & 0.03345 & -8.06 \\
\hline 362.202 & 5.39 & 236.8 & 0.03065 & 0.03343 & -8.30 \\
\hline 361.791 & 4.79 & 187.7 & 0.02692 & 0.02829 & -4.86 \\
\hline 361.765 & 4.79 & 187.8 & 0.02700 & 0.02831 & -4.61 \\
\hline 362.009 & 4.79 & 187.2 & 0.02682 & 0.02824 & -5.04 \\
\hline 362.012 & 4.79 & 187.1 & 0.02683 & 0.02824 & -5.00 \\
\hline 362.247 & 4.79 & 186.4 & 0.02675 & 0.02817 & -5.05 \\
\hline 362.239 & 4.79 & 186.4 & 0.02674 & 0.02817 & -5.07 \\
\hline 362.474 & 4.79 & 185.8 & 0.02677 & 0.02811 & -4.76 \\
\hline 362.464 & 4.79 & 185.8 & 0.02681 & 0.02811 & -4.62 \\
\hline 362.710 & 4.79 & 185.2 & 0.02676 & 0.02805 & -4.60 \\
\hline 362.711 & 4.79 & 185.3 & 0.02674 & 0.02806 & -4.70 \\
\hline 362.139 & 4.03 & 139.6 & 0.02367 & 0.02410 & -1.79 \\
\hline 362.149 & 4.03 & 139.6 & 0.02362 & 0.02410 & -2.01 \\
\hline 362.390 & 4.03 & 139.3 & 0.02375 & 0.02409 & -1.42 \\
\hline 362.375 & 4.03 & 139.4 & 0.02367 & 0.02410 & -1.76 \\
\hline 362.642 & 4.03 & 139.0 & 0.02369 & 0.02408 & -1.62 \\
\hline 362.645 & 4.03 & 139.0 & 0.02362 & 0.02408 & -1.90 \\
\hline 362.939 & 4.03 & 138.5 & 0.02357 & 0.02405 & -2.00 \\
\hline 362.924 & 4.03 & 138.4 & 0.02365 & 0.02405 & -1.65 \\
\hline 363.226 & 4.03 & 138.0 & 0.02371 & 0.02403 & -1.33 \\
\hline 363.240 & 4.03 & 138.0 & 0.02371 & 0.02403 & -1.32 \\
\hline
\end{tabular}


Table 9. Thermal conductivity for R410A (continued)

\begin{tabular}{|c|c|c|c|c|c|}
\hline $\begin{array}{c}\text { Temperature } \\
(\mathrm{K})\end{array}$ & $\begin{array}{c}\text { Pressure } \\
(\mathrm{MPa})\end{array}$ & $\begin{array}{c}\text { Density (calc) } \\
\left(\mathrm{kg} / \mathrm{m}^{3}\right)\end{array}$ & $\begin{array}{c}\lambda_{\exp } \\
(\mathrm{W} / \mathrm{m} \cdot \mathrm{K})\end{array}$ & $\begin{array}{c}\lambda_{\text {calc }} \\
(\mathrm{W} / \mathrm{m} \cdot \mathrm{K})\end{array}$ & $\begin{array}{l}\Delta \lambda \\
(\%)\end{array}$ \\
\hline 316.449 & 29.89 & 1152.6 & 0.09860 & 0.11194 & -11.92 \\
\hline 316.795 & 29.88 & 1151.6 & 0.09857 & 0.11179 & -11.83 \\
\hline 317.142 & 29.84 & 1150.5 & 0.09869 & 0.11161 & -11. \\
\hline 315.892 & 22.08 & 1121.0 & 0.09406 & 0.10603 & -11. \\
\hline 316.164 & 22.08 & 1120.1 & 0.09510 & 0.10590 & -10.20 \\
\hline 316.478 & 22.10 & 1119.2 & 0.09367 & 0.10578 & -11.45 \\
\hline 316.832 & 22.12 & 1118.1 & 0.09389 & 0.10564 & -11.12 \\
\hline 317.183 & 22.13 & 1117.0 & 0.09362 & 0.10549 & -11.25 \\
\hline 315.940 & 15.71 & 1086.8 & 0.08952 & 0.10031 & -10.76 \\
\hline 316.229 & 15.70 & 1085.7 & 0.08908 & 0.10017 & -11.07 \\
\hline 316.540 & 15.70 & 1084.5 & 0.08870 & 0.10002 & -11.31 \\
\hline 316.896 & 15.72 & 1083.3 & 0.08858 & 0.09987 & -11.30 \\
\hline 317.248 & 15.74 & 1082.1 & 0.08897 & 0.09971 & -10.77 \\
\hline 316.012 & 11.17 & 1055.7 & 0.08508 & 0.09564 & -11.05 \\
\hline 316.216 & 11.15 & 1054.7 & 0.08504 & 0.09552 & -10.9 \\
\hline 316.440 & 11.15 & 1053.7 & 0.08478 & 0.09540 & -11.1 \\
\hline 316.685 & 11.15 & 1052.7 & 0.08408 & 0.09528 & -11.76 \\
\hline 316.919 & 11.17 & 1051.9 & 0.08441 & 0.09518 & -11.3 \\
\hline 316.055 & 7.47 & 1023.3 & 0.08098 & 0.09129 & -11.2 \\
\hline 316.264 & 7.49 & 1022.4 & 0.08053 & 0.09119 & -11.69 \\
\hline 316.480 & 7.50 & 1021.4 & 0.08079 & 0.09108 & -11.3 \\
\hline 316.737 & 7.48 & 1020.0 & 0.08031 & 0.09092 & -11.66 \\
\hline 316.994 & 7.47 & 1018.6 & 0.08064 & 0.09076 & -11.1 \\
\hline 316.093 & 4.66 & 990.4 & 0.07711 & 0.08738 & -11.76 \\
\hline 316.245 & 4.66 & 989.4 & 0.07791 & 0.08729 & -10.74 \\
\hline 316.411 & 4.66 & 988.4 & 0.07729 & 0.08718 & -11.35 \\
\hline 316.593 & 4.67 & 987.3 & 0.07683 & 0.08708 & -11.77 \\
\hline 316.773 & 4.68 & 986.4 & 0.07681 & 0.08698 & -11.69 \\
\hline 316.105 & 2.81 & 960.6 & 0.07480 & 0.08432 & -11.29 \\
\hline 316.265 & 2.81 & 959.5 & 0.07512 & 0.08422 & -10.80 \\
\hline 316.457 & 2.82 & 958.0 & 0.07475 & 0.08409 & -11.11 \\
\hline 316.634 & 2.82 & 956.7 & 0.07435 & 0.08398 & -11.46 \\
\hline 316.780 & 2.82 & 955.7 & 0.07476 & 0.08388 & -10.87 \\
\hline 381.680 & 30.09 & 955.9 & 0.08109 & 0.08792 & -7.7 \\
\hline 381.881 & 30.08 & 955.2 & 0.08059 & 0.08785 & -8.26 \\
\hline 382.064 & 30.08 & 954.6 & 0.08048 & 0.08779 & -8.32 \\
\hline 382.259 & 30.09 & 954.1 & 0.08040 & 0.08774 & -8.36 \\
\hline 382.485 & 30.10 & 953.5 & 0.08060 & 0.08768 & -8.07 \\
\hline 381.720 & 23.29 & 897.0 & 0.07433 & 0.08091 & -8.14 \\
\hline 381.932 & 23.28 & 896.1 & 0.07445 & 0.08083 & -7.90 \\
\hline 382.128 & 23.28 & 895.4 & 0.07408 & 0.08076 & -8.28 \\
\hline 382.358 & 23.29 & 894.6 & 0.07430 & 0.08069 & -7.92 \\
\hline 382.577 & 23.30 & 893.9 & 0.07422 & 0.08063 & -7.95 \\
\hline 381.603 & 18.97 & 845.1 & 0.06895 & 0.07568 & -8.90 \\
\hline 381.796 & 18.97 & 844.2 & 0.06918 & 0.07561 & -8.51 \\
\hline 382.005 & 18.96 & 843.2 & 0.06887 & 0.07552 & -8.80 \\
\hline 382.224 & 18.96 & 842.1 & 0.06912 & 0.07543 & -8.37 \\
\hline
\end{tabular}


Table 9. Thermal conductivity for R410A (continued)

\begin{tabular}{|c|c|c|c|c|c|}
\hline $\begin{array}{c}\text { Temperature } \\
(\mathrm{K})\end{array}$ & $\begin{array}{c}\text { Pressure } \\
(\mathrm{MPa})\end{array}$ & $\begin{array}{c}\text { Density (calc) } \\
\left(\mathrm{kg} / \mathrm{m}^{3}\right)\end{array}$ & $\begin{array}{c}\lambda_{\exp } \\
(\mathrm{W} / \mathrm{m} \cdot \mathrm{K})\end{array}$ & $\begin{array}{c}\lambda_{\text {calc }} \\
(\mathrm{W} / \mathrm{m} \cdot \mathrm{K})\end{array}$ & $\begin{array}{l}\Delta \lambda \\
(\%)\end{array}$ \\
\hline 382.454 & 18.96 & 841.1 & 0.06933 & 0.07534 & -7.98 \\
\hline 381.656 & 15.96 & 794.0 & 0.06450 & 0.07129 & -9.52 \\
\hline 381.873 & 15.95 & 792.7 & 0.06465 & 0.07118 & -9.18 \\
\hline 382.092 & 15.95 & 791.5 & 0.06461 & 0.07109 & -9.11 \\
\hline 382.326 & 15.95 & 790.2 & 0.06459 & 0.07099 & -9.01 \\
\hline 382.570 & 15.95 & 788.9 & 0.06488 & 0.07089 & -8.48 \\
\hline 381.765 & 13.69 & 739.5 & 0.06073 & 0.06725 & -9.70 \\
\hline 381.990 & 13.69 & 738.0 & 0.06061 & 0.06715 & -9.73 \\
\hline 382.226 & 13.69 & 736.5 & 0.06048 & 0.06703 & -9.78 \\
\hline 382.465 & 13.70 & 735.0 & 0.06050 & 0.06693 & -9.61 \\
\hline 382.726 & 13.70 & 733.5 & 0.06053 & 0.06682 & -9.41 \\
\hline 381.611 & 12.16 & 689.4 & 0.05792 & 0.06403 & -9.54 \\
\hline 381.614 & 12.16 & 689.5 & 0.05766 & 0.06403 & -9.9 \\
\hline 381.822 & 12.16 & 687.8 & 0.05749 & 0.06391 & -10.05 \\
\hline 381.829 & 12.15 & 687.6 & 0.05746 & 0.06391 & -10.09 \\
\hline 382.057 & 12.15 & 685.8 & 0.05723 & 0.06378 & -10.28 \\
\hline 382.055 & 12.15 & 685.9 & 0.05688 & 0.06378 & -10.82 \\
\hline 382.303 & 12.15 & 683.9 & 0.05726 & 0.06365 & -10.05 \\
\hline 382.307 & 12.15 & 683.9 & 0.05722 & 0.06365 & -10.11 \\
\hline 382.550 & 12.15 & 682.0 & 0.05716 & 0.06352 & -10.02 \\
\hline 382.565 & 12.15 & 681.9 & 0.05720 & 0.06352 & -9.95 \\
\hline 381.468 & 10.97 & 636.3 & 0.05447 & 0.06099 & -10.70 \\
\hline 381.466 & 10.97 & 636.5 & 0.05432 & 0.06100 & -10.95 \\
\hline 381.667 & 10.97 & 634.7 & 0.05442 & 0.06089 & -10.62 \\
\hline 381.666 & 10.97 & 634.8 & 0.05452 & 0.06089 & -10.46 \\
\hline 381.880 & 10.97 & 632.9 & 0.05447 & 0.06077 & -10.37 \\
\hline 381.890 & 10.97 & 632.9 & 0.05442 & 0.06077 & -10.44 \\
\hline 382.123 & 10.97 & 630.7 & 0.05388 & 0.06063 & -11.13 \\
\hline 382.128 & 10.97 & 630.6 & 0.05402 & 0.06062 & -10.89 \\
\hline 382.372 & 10.97 & 628.3 & 0.05427 & 0.06047 & -10.26 \\
\hline 382.387 & 10.97 & 628.1 & 0.05395 & 0.06046 & -10.77 \\
\hline 381.504 & 10.11 & 583.9 & 0.05198 & 0.05823 & -10.73 \\
\hline 381.513 & 10.12 & 583.9 & 0.05184 & 0.05823 & -10.97 \\
\hline 381.720 & 10.12 & 581.9 & 0.05187 & 0.05809 & -10.71 \\
\hline 381.725 & 10.12 & 581.9 & 0.05187 & 0.05809 & -10.71 \\
\hline 381.942 & 10.12 & 579.7 & 0.05168 & 0.05795 & -10.82 \\
\hline 381.943 & 10.12 & 579.8 & 0.05173 & 0.05795 & -10.74 \\
\hline 382.187 & 10.12 & 577.2 & 0.05136 & 0.05779 & -11.13 \\
\hline 382.194 & 10.12 & 577.1 & 0.05142 & 0.05778 & -11.01 \\
\hline 382.452 & 10.12 & 574.4 & 0.05137 & 0.05761 & -10.83 \\
\hline 382.465 & 10.12 & 574.3 & 0.05143 & 0.05760 & -10.71 \\
\hline 381.372 & 9.46 & 534.0 & 0.04979 & 0.05563 & -10.49 \\
\hline 381.364 & 9.46 & 534.1 & 0.04970 & 0.05563 & -10.66 \\
\hline 381.573 & 9.46 & 531.9 & 0.04962 & 0.05548 & -10.56 \\
\hline 381.575 & 9.46 & 532.0 & 0.04918 & 0.05548 & -11.36 \\
\hline 381.792 & 9.46 & 529.7 & 0.04906 & 0.05532 & -11.32 \\
\hline 381.796 & 9.46 & 529.7 & 0.04927 & 0.05532 & -10.94 \\
\hline
\end{tabular}


Table 9. Thermal conductivity for R410A (continued)

\begin{tabular}{|c|c|c|c|c|c|}
\hline $\begin{array}{c}\text { Temperature } \\
\text { (K) }\end{array}$ & $\begin{array}{c}\text { Pressure } \\
(\mathrm{MPa})\end{array}$ & $\begin{array}{c}\text { Density (calc) } \\
\left(\mathrm{kg} / \mathrm{m}^{3}\right)\end{array}$ & $\begin{array}{c}\lambda_{\exp } \\
(\mathrm{W} / \mathrm{m} \cdot \mathrm{K})\end{array}$ & $\begin{array}{c}\lambda_{\text {calc }} \\
(\mathrm{W} / \mathrm{m} \cdot \mathrm{K})\end{array}$ & $\begin{array}{l}\Delta \lambda \\
(\%)\end{array}$ \\
\hline 382.025 & 9.46 & 527.3 & 0.04901 & 0.05515 & -11.14 \\
\hline 382.047 & 9.46 & 527.1 & 0.04908 & 0.05514 & -10.99 \\
\hline 382.295 & 9.46 & 524.5 & 0.04897 & 0.05496 & -10.89 \\
\hline 382.295 & 9.46 & 524.5 & 0.04899 & 0.05496 & -10.86 \\
\hline 381.475 & 9.47 & 533.8 & 0.04964 & 0.05560 & -10.71 \\
\hline 381.479 & 9.47 & 533.7 & 0.04934 & 0.05559 & -11.24 \\
\hline 381.681 & 9.47 & 531.5 & 0.04932 & 0.05544 & -11.04 \\
\hline 381.687 & 9.46 & 531.4 & 0.04912 & 0.05543 & -11.39 \\
\hline 381.905 & 9.47 & 529.0 & 0.04919 & 0.05527 & -11.00 \\
\hline 381.911 & 9.47 & 529.1 & 0.04917 & 0.05527 & -11.04 \\
\hline 382.137 & 9.47 & 526.7 & 0.04891 & 0.05510 & -11.24 \\
\hline 382.134 & 9.47 & 526.8 & 0.04895 & 0.05511 & -11.18 \\
\hline 382.383 & 9.47 & 524.1 & 0.04880 & 0.05492 & -11.15 \\
\hline 382.389 & 9.47 & 524.1 & 0.04884 & 0.05492 & -11.07 \\
\hline 381.301 & 8.97 & 490.0 & 0.04688 & 0.05313 & -11.76 \\
\hline 381.300 & 8.97 & 490.0 & 0.04714 & 0.05313 & -11.28 \\
\hline 381.497 & 8.97 & 487.8 & 0.04715 & 0.05296 & -10.97 \\
\hline 381.511 & 8.97 & 487.5 & 0.04718 & 0.05294 & -10.89 \\
\hline 381.725 & 8.97 & 485.2 & 0.04655 & 0.05276 & -11.78 \\
\hline 381.739 & 8.97 & 485.0 & 0.04695 & 0.05275 & -11.00 \\
\hline 381.964 & 8.97 & 482.6 & 0.04655 & 0.05256 & -11.44 \\
\hline 381.953 & 8.97 & 482.7 & 0.04644 & 0.05257 & -11.66 \\
\hline 382.211 & 8.97 & 479.9 & 0.04654 & 0.05236 & -11.11 \\
\hline 382.200 & 8.97 & 480.2 & 0.04641 & 0.05237 & -11.39 \\
\hline 381.171 & 8.46 & 439.6 & 0.04397 & 0.04987 & -11.83 \\
\hline 381.174 & 8.46 & 439.6 & 0.04407 & 0.04987 & -11.63 \\
\hline 381.364 & 8.46 & 437.8 & 0.04414 & 0.04971 & -11.20 \\
\hline 381.365 & 8.46 & 437.8 & 0.04430 & 0.04971 & -10.88 \\
\hline 381.583 & 8.46 & 435.6 & 0.04377 & 0.04952 & -11.61 \\
\hline 381.585 & 8.46 & 435.5 & 0.04386 & 0.04951 & -11.41 \\
\hline 381.820 & 8.46 & 433.1 & 0.04380 & 0.04930 & -11.16 \\
\hline 381.822 & 8.46 & 433.1 & 0.04374 & 0.04930 & -11.28 \\
\hline 382.063 & 8.46 & 430.7 & 0.04390 & 0.04909 & -10.58 \\
\hline 382.053 & 8.46 & 430.8 & 0.04369 & 0.04910 & -11.02 \\
\hline 381.243 & 7.98 & 387.8 & 0.04094 & 0.04596 & -10.92 \\
\hline 381.250 & 7.98 & 387.8 & 0.04085 & 0.04595 & -11.11 \\
\hline 381.446 & 7.98 & 386.2 & 0.04088 & 0.04580 & -10.74 \\
\hline 381.458 & 7.98 & 386.2 & 0.04069 & 0.04580 & -11.15 \\
\hline 381.693 & 7.98 & 384.3 & 0.04060 & 0.04561 & -10.99 \\
\hline 381.690 & 7.98 & 384.4 & 0.04053 & 0.04562 & -11.16 \\
\hline 381.937 & 7.98 & 382.4 & 0.04063 & 0.04542 & -10.55 \\
\hline 381.940 & 7.98 & 382.4 & 0.04056 & 0.04542 & -10.71 \\
\hline 382.216 & 7.98 & 380.1 & 0.04061 & 0.04520 & -10.15 \\
\hline 382.233 & 7.98 & 379.9 & 0.04059 & 0.04518 & -10.16 \\
\hline 381.188 & 7.47 & 337.7 & 0.03770 & 0.04174 & -9.68 \\
\hline 381.192 & 7.47 & 337.7 & 0.03777 & 0.04174 & -9.51 \\
\hline 381.324 & 7.47 & 336.9 & 0.03726 & 0.04165 & -10.55 \\
\hline
\end{tabular}


Table 9. Thermal conductivity for R410A (continued)

\begin{tabular}{|c|c|c|c|c|c|}
\hline $\begin{array}{l}\text { Temperature } \\
\text { (K) }\end{array}$ & $\begin{array}{l}\text { Pressure } \\
(\mathrm{MPa})\end{array}$ & $\begin{array}{c}\text { Density (calc) } \\
\left(\mathrm{kg} / \mathrm{m}^{3}\right)\end{array}$ & $\begin{array}{c}\lambda_{\exp } \\
(\mathrm{W} / \mathrm{m} \cdot \mathrm{K})\end{array}$ & $\begin{array}{c}\lambda_{\text {calc }} \\
(\mathrm{W} / \mathrm{m} \cdot \mathrm{K})\end{array}$ & $\begin{array}{l}\Delta \lambda \\
(\%)\end{array}$ \\
\hline 381.325 & 7.47 & 336.9 & 0.03714 & 0.04165 & -10.84 \\
\hline 381.459 & 7.47 & 336.0 & 0.03777 & 0.04157 & -9.13 \\
\hline 381.468 & 7.47 & 336.0 & 0.03740 & 0.04156 & -10.01 \\
\hline 381.606 & 7.48 & 335.1 & 0.03731 & 0.04147 & -10.03 \\
\hline 381.604 & 7.48 & 335.1 & 0.03733 & 0.04147 & -9.99 \\
\hline 381.758 & 7.48 & 334.1 & 0.03722 & 0.04137 & -10.03 \\
\hline 381.762 & 7.47 & 334.1 & 0.03716 & 0.04136 & -10.15 \\
\hline 381.283 & 6.98 & 292.5 & 0.03421 & 0.03767 & -9.18 \\
\hline 381.302 & 6.98 & 292.4 & 0.03422 & 0.03766 & -9.14 \\
\hline 381.441 & 6.98 & 291.8 & 0.03477 & 0.03759 & -7.51 \\
\hline 381.439 & 6.98 & 291.8 & 0.03460 & 0.03760 & -7.97 \\
\hline 381.584 & 6.98 & 291.1 & 0.03437 & 0.03752 & -8.41 \\
\hline 381.597 & 6.98 & 291.1 & 0.03430 & 0.03752 & -8.58 \\
\hline 381.757 & 6.98 & 290.3 & 0.03424 & 0.03744 & -8.54 \\
\hline 381.757 & 6.98 & 290.3 & 0.03417 & 0.03744 & -8.73 \\
\hline 381.931 & 6.98 & 289.5 & 0.03427 & 0.03735 & -8.25 \\
\hline 381.937 & 6.98 & 289.5 & 0.03425 & 0.03735 & -8.30 \\
\hline 381.456 & 6.32 & 241.5 & 0.03088 & 0.03303 & -6.52 \\
\hline 381.635 & 6.32 & 240.9 & 0.03110 & 0.03298 & -5.70 \\
\hline 381.813 & 6.32 & 240.4 & 0.03069 & 0.03293 & -6.80 \\
\hline 381.996 & 6.32 & 239.8 & 0.03080 & 0.03288 & -6.32 \\
\hline 382.183 & 6.32 & 239.3 & 0.03093 & 0.03282 & -5.77 \\
\hline 381.626 & 5.56 & 193.1 & 0.02810 & 0.02892 & -2.83 \\
\hline 381.815 & 5.56 & 192.8 & 0.02816 & 0.02890 & -2.55 \\
\hline 382.037 & 5.56 & 192.4 & 0.02778 & 0.02887 & -3.78 \\
\hline 382.253 & 5.56 & 192.0 & 0.02786 & 0.02884 & -3.40 \\
\hline 382.513 & 5.56 & 191.3 & 0.02781 & 0.02879 & -3.42 \\
\hline 381.888 & 4.56 & 142.6 & 0.02501 & 0.02526 & -0.98 \\
\hline 382.127 & 4.56 & 142.4 & 0.02501 & 0.02525 & -0.96 \\
\hline 382.371 & 4.56 & 142.0 & 0.02522 & 0.02524 & -0.09 \\
\hline 382.626 & 4.55 & 141.6 & 0.02510 & 0.02523 & -0.53 \\
\hline 382.912 & 4.55 & 141.3 & 0.02496 & 0.02523 & -1.07 \\
\hline 331.276 & 22.24 & 1070.7 & 0.08902 & 0.09941 & -10.45 \\
\hline 331.486 & 22.25 & 1070.1 & 0.08858 & 0.09933 & -10.82 \\
\hline 331.704 & 22.24 & 1069.3 & 0.08772 & 0.09923 & -11.60 \\
\hline 331.946 & 22.22 & 1068.3 & 0.08837 & 0.09911 & -10.83 \\
\hline 332.199 & 22.21 & 1067.4 & 0.08790 & 0.09899 & -11.21 \\
\hline 331.295 & 20.30 & 1059.1 & 0.08683 & 0.09762 & -11.05 \\
\hline 331.498 & 20.32 & 1058.4 & 0.08710 & 0.09754 & -10.71 \\
\hline 331.717 & 20.32 & 1057.7 & 0.08650 & 0.09745 & -11.24 \\
\hline 331.960 & 20.30 & 1056.7 & 0.08644 & 0.09732 & -11.18 \\
\hline 332.215 & 20.29 & 1055.7 & 0.08645 & 0.09720 & -11.06 \\
\hline 331.287 & 16.18 & 1030.9 & 0.08336 & 0.09354 & -10.89 \\
\hline 331.501 & 16.18 & 1030.0 & 0.08345 & 0.09344 & -10.69 \\
\hline 331.744 & 16.20 & 1029.2 & 0.08273 & 0.09335 & -11.38 \\
\hline 331.992 & 16.21 & 1028.3 & 0.08271 & 0.09325 & -11.30 \\
\hline 332.254 & 16.21 & 1027.2 & 0.08271 & 0.09313 & -11.19 \\
\hline
\end{tabular}


Table 9. Thermal conductivity for R410A (continued)

\begin{tabular}{|c|c|c|c|c|c|}
\hline $\begin{array}{c}\text { Temperature } \\
\text { (K) }\end{array}$ & $\begin{array}{c}\text { Pressure } \\
(\mathrm{MPa})\end{array}$ & $\begin{array}{c}\text { Density (calc) } \\
\left(\mathrm{kg} / \mathrm{m}^{3}\right)\end{array}$ & $\begin{array}{c}\lambda_{\exp } \\
(\mathrm{W} / \mathrm{m} \cdot \mathrm{K})\end{array}$ & $\begin{array}{c}\lambda_{\text {calc }} \\
(\mathrm{W} / \mathrm{m} \cdot \mathrm{K})\end{array}$ & $\begin{array}{l}\Delta \lambda \\
(\%)\end{array}$ \\
\hline 331.308 & 12.66 & 1001.0 & 0.07949 & 0.08963 & -11.31 \\
\hline 331.542 & 12.65 & 999.8 & 0.07968 & 0.08950 & -10.97 \\
\hline 331.766 & 12.65 & 998.8 & 0.07858 & 0.08939 & -12.09 \\
\hline 332.028 & 12.66 & 997.7 & 0.07957 & 0.08928 & -10.87 \\
\hline 332.301 & 12.67 & 996.6 & 0.07912 & 0.08916 & -11.26 \\
\hline 331.036 & 9.74 & 971.1 & 0.07676 & 0.08610 & -10.85 \\
\hline 331.242 & 9.73 & 969.9 & 0.07682 & 0.08598 & -10.66 \\
\hline 331.474 & 9.73 & 968.7 & 0.07636 & 0.08586 & -11.06 \\
\hline 331.720 & 9.74 & 967.4 & 0.07618 & 0.08574 & -11.15 \\
\hline 331.965 & 9.75 & 966.3 & 0.07617 & 0.08563 & -11.04 \\
\hline 331.055 & 7.31 & 936.7 & 0.07319 & 0.08256 & -11.35 \\
\hline 331.280 & 7.31 & 935.3 & 0.07332 & 0.08244 & -11.06 \\
\hline 331.503 & 7.31 & 933.9 & 0.07324 & 0.08231 & -11.02 \\
\hline 331.748 & 7.30 & 932.2 & 0.07268 & 0.08216 & -11.53 \\
\hline 332.032 & 7.30 & 930.3 & 0.07273 & 0.08199 & -11.29 \\
\hline 330.936 & 5.64 & 905.3 & 0.07044 & 0.07978 & -11.71 \\
\hline 330.945 & 5.64 & 905.2 & 0.07073 & 0.07978 & -11.34 \\
\hline 331.088 & 5.64 & 904.1 & 0.07060 & 0.07969 & -11.40 \\
\hline 331.087 & 5.64 & 904.1 & 0.07017 & 0.07969 & -11.94 \\
\hline 331.250 & 5.64 & 902.8 & 0.06994 & 0.07959 & -12.12 \\
\hline 331.256 & 5.64 & 902.9 & 0.07000 & 0.07959 & -12.05 \\
\hline 331.403 & 5.65 & 901.8 & 0.07064 & 0.07951 & -11.15 \\
\hline 331.397 & 5.65 & 901.9 & 0.07049 & 0.07952 & -11.35 \\
\hline 331.576 & 5.65 & 900.5 & 0.07028 & 0.07941 & -11.49 \\
\hline 331.562 & 5.66 & 900.7 & 0.07086 & 0.07942 & -10.78 \\
\hline 330.827 & 4.38 & 871.3 & 0.06715 & 0.07731 & -13.14 \\
\hline 330.847 & 4.38 & 871.1 & 0.06683 & 0.07730 & -13.54 \\
\hline 330.963 & 4.38 & 869.9 & 0.06823 & 0.07722 & -11.64 \\
\hline 330.974 & 4.38 & 869.8 & 0.06784 & 0.07722 & -12.14 \\
\hline 331.109 & 4.39 & 868.5 & 0.06777 & 0.07713 & -12.14 \\
\hline 331.109 & 4.39 & 868.6 & 0.06753 & 0.07714 & -12.45 \\
\hline 331.257 & 4.39 & 867.1 & 0.06773 & 0.07703 & -12.08 \\
\hline 331.262 & 4.39 & 867.1 & 0.06764 & 0.07703 & -12.19 \\
\hline 331.419 & 4.39 & 865.4 & 0.06831 & 0.07692 & -11.20 \\
\hline 331.430 & 4.39 & 865.3 & 0.06785 & 0.07692 & -11.79 \\
\hline 330.836 & 3.73 & 844.3 & 0.06515 & 0.07582 & -14.07 \\
\hline 330.847 & 3.73 & 844.2 & 0.06504 & 0.07581 & -14.21 \\
\hline 330.978 & 3.73 & 842.3 & 0.06600 & 0.07571 & -12.83 \\
\hline 330.962 & 3.73 & 842.5 & 0.06643 & 0.07572 & -12.27 \\
\hline 331.110 & 3.73 & 840.3 & 0.06634 & 0.07561 & -12.26 \\
\hline 331.109 & 3.73 & 840.3 & 0.06672 & 0.07561 & -11.76 \\
\hline 331.265 & 3.73 & 838.1 & 0.06625 & 0.07550 & -12.25 \\
\hline 331.277 & 3.73 & 837.9 & 0.06616 & 0.07549 & -12.36 \\
\hline 331.434 & 3.73 & 835.7 & 0.06647 & 0.07538 & -11.82 \\
\hline 331.444 & 3.73 & 835.7 & 0.06650 & 0.07538 & -11.78 \\
\hline
\end{tabular}


Table 9. Thermal conductivity for R410A (continued)

\begin{tabular}{|c|c|c|c|c|c|}
\hline $\begin{array}{l}\text { Temperature } \\
\text { (K) }\end{array}$ & $\begin{array}{c}\text { Pressure } \\
(\mathrm{MPa})\end{array}$ & $\begin{array}{c}\text { Density (calc) } \\
\left(\mathrm{kg} / \mathrm{m}^{3}\right)\end{array}$ & $\begin{array}{c}\lambda_{\exp } \\
(\mathrm{W} / \mathrm{m} \cdot \mathrm{K})\end{array}$ & $\begin{array}{c}\lambda_{\text {calc }} \\
(\mathrm{W} / \mathrm{m} \cdot \mathrm{K})\end{array}$ & $\begin{array}{l}\Delta \lambda \\
(\%\end{array}$ \\
\hline 401.352 & 25.08 & 844.2 & 0.07125 & 0.07637 & -6.71 \\
\hline 401.586 & 25.09 & 843.5 & 0.07052 & 0.07631 & -7.59 \\
\hline 401.840 & 25.10 & 842.6 & 0.07074 & 0.07623 & -7.20 \\
\hline 402.114 & 25.09 & 841.6 & 0.07057 & 0.07614 & -7.32 \\
\hline 402.413 & 25.08 & 840.3 & 0.07051 & 0.07603 & -7.26 \\
\hline 401.338 & 21.50 & 797.4 & 0.06662 & 0.07190 & -7.34 \\
\hline 401.587 & 21.50 & 796.5 & 0.06656 & 0.07182 & -7.32 \\
\hline 401.870 & 21.51 & 795.4 & 0.06628 & 0.07173 & -7.59 \\
\hline 402.164 & 21.50 & 794.1 & 0.06597 & 0.07162 & -7.89 \\
\hline 402.482 & 21.49 & 792.7 & 0.06637 & 0.07150 & -7.17 \\
\hline 401.393 & 18.47 & 744.7 & 0.06213 & 0.06745 & -7.89 \\
\hline 401.652 & 18.47 & 743.5 & 0.06251 & 0.06736 & -7.20 \\
\hline 401.927 & 18.46 & 742.0 & 0.06249 & 0.06724 & -7.07 \\
\hline 402.242 & 18.46 & 740.5 & 0.06202 & 0.06712 & -7.60 \\
\hline 402.592 & 18.46 & 738.9 & 0.06216 & 0.06700 & -7.22 \\
\hline 401.244 & 16.30 & 695.5 & 0.05906 & 0.06376 & -7.38 \\
\hline 401.260 & 16.30 & 695.3 & 0.05910 & 0.06375 & -7.29 \\
\hline 401.437 & 16.30 & 694.3 & 0.05880 & 0.06368 & -7.66 \\
\hline 401.440 & 16.29 & 694.3 & 0.05938 & 0.06367 & -6.74 \\
\hline 401.642 & 16.29 & 693.2 & 0.05880 & 0.06359 & -7.53 \\
\hline 401.634 & 16.29 & 693.2 & 0.05871 & 0.06360 & -7.68 \\
\hline 401.845 & 16.30 & 692.2 & 0.05880 & 0.06352 & -7.42 \\
\hline 401.848 & 16.30 & 692.2 & 0.05886 & 0.06352 & -7.34 \\
\hline 402.058 & 16.30 & 691.1 & 0.05875 & 0.06344 & -7.39 \\
\hline 402.054 & 16.30 & 691.2 & 0.05869 & 0.06344 & -7.49 \\
\hline 401.285 & 14.48 & 640.2 & 0.05587 & 0.06001 & -6.89 \\
\hline 401.298 & 14.48 & 640.1 & 0.05567 & 0.06000 & -7.21 \\
\hline 401.493 & 14.48 & 638.9 & 0.05580 & 0.05991 & -6.86 \\
\hline 401.488 & 14.48 & 638.9 & 0.05582 & 0.05991 & -6.83 \\
\hline 401.699 & 14.48 & 637.6 & 0.05526 & 0.05982 & -7.62 \\
\hline 401.709 & 14.48 & 637.5 & 0.05532 & 0.05982 & -7.52 \\
\hline 401.913 & 14.48 & 636.3 & 0.05526 & 0.05973 & -7.48 \\
\hline 401.908 & 14.48 & 636.5 & 0.05515 & 0.05974 & -7.68 \\
\hline 402.133 & 14.48 & 635.2 & 0.05519 & 0.05965 & -7.47 \\
\hline 402.130 & 14.48 & 635.2 & 0.05524 & 0.05965 & -7.39 \\
\hline 401.353 & 13.30 & 594.7 & 0.05310 & 0.05712 & -7.04 \\
\hline 401.350 & 13.30 & 594.7 & 0.05262 & 0.05712 & -7.88 \\
\hline 401.537 & 13.30 & 593.4 & 0.05259 & 0.05703 & -7.79 \\
\hline 401.533 & 13.30 & 593.4 & 0.05262 & 0.05704 & -7.74 \\
\hline 401.740 & 13.30 & 592.1 & 0.05259 & 0.05694 & -7.64 \\
\hline 401.751 & 13.30 & 592.1 & 0.05253 & 0.05694 & -7.74 \\
\hline 401.983 & 13.30 & 590.7 & 0.05256 & 0.05684 & -7.53 \\
\hline 401.977 & 13.31 & 590.8 & 0.05269 & 0.05684 & -7.31 \\
\hline 402.220 & 13.31 & 589.2 & 0.05248 & 0.05674 & -7.50 \\
\hline 402.224 & 13.31 & 589.2 & 0.05240 & 0.05674 & -7.64 \\
\hline 401.208 & 12.16 & 542.4 & 0.05011 & 0.05394 & -7.11 \\
\hline 401.205 & 12.16 & 542.5 & 0.05015 & 0.05395 & -7.04 \\
\hline 401.398 & 12.17 & 541.3 & 0.04993 & 0.05386 & -7.29 \\
\hline
\end{tabular}


Table 9. Thermal conductivity for R410A (continued)

\begin{tabular}{|c|c|c|c|c|c|}
\hline $\begin{array}{c}\text { Temperature } \\
(\mathrm{K})\end{array}$ & $\begin{array}{c}\text { Pressure } \\
(\mathrm{MPa})\end{array}$ & $\begin{array}{c}\text { Density (calc) } \\
\left(\mathrm{kg} / \mathrm{m}^{3}\right)\end{array}$ & $\begin{array}{c}\lambda_{\exp } \\
(\mathrm{W} / \mathrm{m} \cdot \mathrm{K})\end{array}$ & $\begin{array}{c}\lambda_{\text {calc }} \\
(\mathrm{W} / \mathrm{m} \cdot \mathrm{K})\end{array}$ & $\begin{array}{l}\Delta \lambda \\
(\%)\end{array}$ \\
\hline 401.388 & 12.17 & 541.3 & 0.05008 & 0.05386 & -7.02 \\
\hline 401.592 & 12.16 & 539.8 & 0.04937 & 0.05376 & -8.16 \\
\hline 401.615 & 12.16 & 539.6 & 0.04967 & 0.05374 & -7.58 \\
\hline 401.842 & 12.16 & 538.1 & 0.04960 & 0.05363 & -7.52 \\
\hline 401.836 & 12.16 & 538.1 & 0.04968 & 0.05363 & -7.37 \\
\hline 402.079 & 12.16 & 536.5 & 0.04986 & 0.05352 & -6.83 \\
\hline 402.089 & 12.16 & 536.5 & 0.04974 & 0.05352 & -7.05 \\
\hline 401.311 & 11.20 & 488.5 & 0.04685 & 0.05062 & -7.45 \\
\hline 401.331 & 11.20 & 488.4 & 0.04671 & 0.05061 & -7.71 \\
\hline 401.513 & 11.20 & 487.3 & 0.04699 & 0.05053 & -7.00 \\
\hline 401.511 & 11.20 & 487.2 & 0.04694 & 0.05052 & -7.09 \\
\hline 401.739 & 11.20 & 485.6 & 0.04639 & 0.05040 & -7.96 \\
\hline 401.746 & 11.20 & 485.5 & 0.04665 & 0.05040 & -7.43 \\
\hline 401.981 & 11.20 & 483.9 & 0.04659 & 0.05028 & -7.34 \\
\hline 401.989 & 11.20 & 483.9 & 0.04666 & 0.05027 & -7.18 \\
\hline 402.250 & 11.20 & 482.2 & 0.04654 & 0.05014 & -7.19 \\
\hline 402.239 & 11.20 & 482.3 & 0.04687 & 0.05016 & -6.55 \\
\hline 401.383 & 10.44 & 441.0 & 0.04416 & 0.04756 & -7.14 \\
\hline 401.400 & 10.44 & 441.0 & 0.04451 & 0.04755 & -6.40 \\
\hline 401.605 & 10.44 & 439.8 & 0.04448 & 0.04746 & -6.2 \\
\hline 401.604 & 10.45 & 439.9 & 0.04430 & 0.04746 & -6.67 \\
\hline 401.810 & 10.45 & 438.7 & 0.04414 & 0.04737 & -6.81 \\
\hline 401.819 & 10.45 & 438.7 & 0.04396 & 0.04736 & -7.19 \\
\hline 402.075 & 10.45 & 437.1 & 0.04413 & 0.04724 & -6.58 \\
\hline 402.068 & 10.45 & 437.2 & 0.04417 & 0.04724 & -6.50 \\
\hline 402.354 & 10.45 & 435.3 & 0.04391 & 0.04710 & -6.76 \\
\hline 402.358 & 10.44 & 435.2 & 0.04386 & 0.04709 & -6.86 \\
\hline 401.459 & 9.72 & 393.4 & 0.04164 & 0.04428 & -5.97 \\
\hline 401.464 & 9.72 & 393.3 & 0.04180 & 0.04427 & -5.59 \\
\hline 401.698 & 9.71 & 391.9 & 0.04140 & 0.04416 & -6.25 \\
\hline 401.696 & 9.71 & 391.9 & 0.04157 & 0.04416 & -5.86 \\
\hline 401.937 & 9.71 & 390.5 & 0.04118 & 0.04405 & -6.51 \\
\hline 401.943 & 9.71 & 390.5 & 0.04111 & 0.04404 & -6.66 \\
\hline 402.210 & 9.71 & 389.2 & 0.04130 & 0.04393 & -5.99 \\
\hline 402.208 & 9.72 & 389.3 & 0.04118 & 0.04394 & -6.28 \\
\hline 402.512 & 9.72 & 387.7 & 0.04109 & 0.04381 & -6.20 \\
\hline 402.508 & 9.72 & 387.8 & 0.04091 & 0.04381 & -6.62 \\
\hline 401.331 & 8.93 & 343.0 & 0.03832 & 0.04061 & -5.64 \\
\hline 401.338 & 8.93 & 342.9 & 0.03843 & 0.04060 & -5.35 \\
\hline 401.554 & 8.93 & 341.9 & 0.03842 & 0.04052 & -5.18 \\
\hline 401.560 & 8.93 & 341.9 & 0.03825 & 0.04051 & -5.58 \\
\hline 401.836 & 8.93 & 340.7 & 0.03836 & 0.04041 & -5.07 \\
\hline 401.836 & 8.94 & 340.8 & 0.03799 & 0.04042 & -6.01 \\
\hline 402.109 & 8.94 & 339.7 & 0.03824 & 0.04032 & -5.16 \\
\hline 402.109 & 8.94 & 339.8 & 0.03795 & 0.04032 & -5.89 \\
\hline 402.416 & 8.94 & 338.5 & 0.03808 & 0.04022 & -5.31 \\
\hline 402.410 & 8.94 & 338.5 & 0.03801 & 0.04022 & -5.49 \\
\hline 401.441 & 8.13 & 292.9 & 0.03510 & 0.03679 & -4.60 \\
\hline
\end{tabular}


Table 9. Thermal conductivity for R410A (continued)

\begin{tabular}{|c|c|c|c|c|c|}
\hline $\begin{array}{c}\text { Temperature } \\
\text { (K) }\end{array}$ & $\begin{array}{c}\text { Pressure } \\
(\mathrm{MPa})\end{array}$ & $\begin{array}{c}\text { Density (calc) } \\
\left(\mathrm{kg} / \mathrm{m}^{3}\right)\end{array}$ & $\begin{array}{c}\lambda_{\exp } \\
(\mathrm{W} / \mathrm{m} \cdot \mathrm{K})\end{array}$ & $\begin{array}{c}\lambda_{\text {calc }} \\
(\mathrm{W} / \mathrm{m} \cdot \mathrm{K})\end{array}$ & $\begin{array}{l}\Delta \lambda \\
(\%)\end{array}$ \\
\hline 401.452 & 8.13 & 292.9 & 0.03523 & 0.03679 & -4.23 \\
\hline 401.698 & 8.13 & 292.0 & 0.03513 & 0.03671 & -4.32 \\
\hline 401.697 & 8.14 & 292.1 & 0.03518 & 0.03672 & -4.20 \\
\hline 401.983 & 8.14 & 291.3 & 0.03483 & 0.03665 & -4.97 \\
\hline 401.995 & 8.14 & 291.3 & 0.03487 & 0.03665 & -4.86 \\
\hline 402.302 & 8.14 & 290.3 & 0.03463 & 0.03657 & -5.31 \\
\hline 402.310 & 8.14 & 290.3 & 0.03473 & 0.03657 & -5.04 \\
\hline 402.648 & 8.14 & 289.2 & 0.03484 & 0.03648 & -4.49 \\
\hline 402.668 & 8.14 & 289.0 & 0.03482 & 0.03646 & -4.51 \\
\hline 401.597 & 7.24 & 242.3 & 0.03243 & 0.03295 & -1.58 \\
\hline 401.598 & 7.24 & 242.3 & 0.03246 & 0.03295 & -1.49 \\
\hline 401.887 & 7.24 & 241.7 & 0.03235 & 0.03291 & -1.70 \\
\hline 401.873 & 7.24 & 241.8 & 0.03255 & 0.03292 & -1.12 \\
\hline 402.189 & 7.24 & 241.2 & 0.03213 & 0.03287 & -2.26 \\
\hline 402.194 & 7.24 & 241.2 & 0.03195 & 0.03287 & -2.81 \\
\hline 402.554 & 7.25 & 240.4 & 0.03202 & 0.03282 & -2.44 \\
\hline 402.543 & 7.24 & 240.4 & 0.03199 & 0.03282 & -2.52 \\
\hline 402.951 & 7.24 & 239.3 & 0.03214 & 0.03274 & -1.85 \\
\hline 402.953 & 7.24 & 239.3 & 0.03201 & 0.03274 & -2.23 \\
\hline 401.794 & 6.17 & 189.9 & 0.02941 & 0.02927 & 0.47 \\
\hline 401.798 & 6.17 & 190.0 & 0.02933 & 0.02928 & 0.18 \\
\hline 402.129 & 6.17 & 189.5 & 0.02921 & 0.02926 & -0.17 \\
\hline 402.132 & 6.17 & 189.6 & 0.02922 & 0.02926 & -0.15 \\
\hline 402.485 & 6.17 & 189.1 & 0.02919 & 0.02924 & -0.19 \\
\hline 402.484 & 6.17 & 189.0 & 0.02931 & 0.02924 & 0.23 \\
\hline 402.908 & 6.17 & 188.3 & 0.02924 & 0.02921 & 0.10 \\
\hline 402.917 & 6.17 & 188.2 & 0.02916 & 0.02921 & -0.16 \\
\hline 403.337 & 6.17 & 187.6 & 0.02913 & 0.02918 & -0.18 \\
\hline 403.366 & 6.17 & 187.5 & 0.02918 & 0.02918 & 0.00 \\
\hline 401.666 & 4.99 & 141.6 & 0.02679 & 0.02637 & 1.60 \\
\hline 401.671 & 4.99 & 141.6 & 0.02698 & 0.02637 & 2.31 \\
\hline 401.885 & 4.99 & 141.5 & 0.02668 & 0.02638 & 1.15 \\
\hline 401.874 & 4.99 & 141.4 & 0.02677 & 0.02637 & 1.51 \\
\hline 402.096 & 4.99 & 141.1 & 0.02656 & 0.02637 & 0.72 \\
\hline 402.098 & 4.98 & 141.0 & 0.02668 & 0.02637 & 1.19 \\
\hline 402.332 & 4.98 & 140.8 & 0.02658 & 0.02637 & 0.80 \\
\hline 402.332 & 4.98 & 140.7 & 0.02674 & 0.02637 & 1.41 \\
\hline 402.581 & 4.98 & 140.6 & 0.02679 & 0.02637 & 1.57 \\
\hline 402.583 & 4.99 & 140.7 & 0.02679 & 0.02638 & 1.55 \\
\hline
\end{tabular}


Table 10. Thermal conductivity for R507A measured by the steady-state technique; data are listed in the order of measurement.

\begin{tabular}{|c|c|c|c|c|c|}
\hline $\begin{array}{c}\text { Temperature } \\
\text { (K) }\end{array}$ & $\begin{array}{c}\text { Pressure } \\
(\mathrm{MPa})\end{array}$ & $\begin{array}{c}\text { Density (calc) } \\
\left(\mathrm{kg} / \mathrm{m}^{3}\right)\end{array}$ & $\begin{array}{c}\lambda_{\exp } \\
(\mathrm{W} / \mathrm{m} \cdot \mathrm{K})\end{array}$ & $\begin{array}{c}\lambda_{\text {calc }} \\
(\mathrm{W} / \mathrm{m} \cdot \mathrm{K})\end{array}$ & $\begin{array}{l}\Delta \lambda \\
(\%)\end{array}$ \\
\hline 362.556 & 0.641 & 22.23 & 0.02048 & 0.02314 & -11.49 \\
\hline 362.845 & 0.642 & 22.21 & 0.02056 & 0.02317 & -11.26 \\
\hline 363.135 & 0.642 & 22.20 & 0.02061 & 0.02320 & -11.16 \\
\hline 363.441 & 0.641 & 22.16 & 0.02069 & 0.02323 & -10.94 \\
\hline 363.782 & 0.641 & 22.11 & 0.02081 & 0.02327 & -10.56 \\
\hline 362.627 & 0.299 & 10.05 & 0.02022 & 0.02313 & -12.58 \\
\hline 362.914 & 0.299 & 10.03 & 0.02019 & 0.02316 & -12.82 \\
\hline 363.241 & 0.299 & 10.04 & 0.02016 & 0.02319 & -13.08 \\
\hline 363.592 & 0.300 & 10.05 & 0.02016 & 0.02323 & -13.22 \\
\hline 363.941 & 0.300 & 10.05 & 0.02016 & 0.02327 & -13.35 \\
\hline 362.494 & 0.208 & 6.95 & 0.02010 & 0.02311 & -13.04 \\
\hline 362.714 & 0.209 & 6.96 & 0.02007 & 0.02314 & -13.25 \\
\hline 362.928 & 0.209 & 6.96 & 0.02008 & 0.02316 & -13.29 \\
\hline 363.167 & 0.209 & 6.95 & 0.02007 & 0.02318 & -13.43 \\
\hline 363.421 & 0.208 & 6.93 & 0.02010 & 0.02321 & -13.40 \\
\hline 362.502 & 0.109 & 3.61 & 0.01990 & 0.02311 & -13.90 \\
\hline 362.719 & 0.109 & 3.61 & 0.01985 & 0.02314 & -14.20 \\
\hline 362.947 & 0.109 & 3.61 & 0.01986 & 0.02316 & -14.25 \\
\hline 363.191 & 0.109 & 3.60 & 0.01987 & 0.02318 & -14.30 \\
\hline 363.439 & 0.108 & 3.58 & 0.01989 & 0.02321 & -14.31 \\
\hline 382.073 & 0.626 & 20.37 & 0.02258 & 0.02519 & -10.37 \\
\hline 382.279 & 0.627 & 20.37 & 0.02257 & 0.02522 & -10.49 \\
\hline 382.523 & 0.627 & 20.37 & 0.02262 & 0.02524 & -10.38 \\
\hline 382.778 & 0.627 & 20.35 & 0.02264 & 0.02527 & -10.40 \\
\hline 381.739 & 0.360 & 11.49 & 0.02247 & 0.02515 & -10.65 \\
\hline 381.944 & 0.360 & 11.50 & 0.02238 & 0.02517 & -11.08 \\
\hline 382.177 & 0.361 & 11.50 & 0.02232 & 0.02519 & -11.41 \\
\hline 382.393 & 0.361 & 11.50 & 0.02234 & 0.02522 & -11.41 \\
\hline 382.672 & 0.361 & 11.49 & 0.02235 & 0.02525 & -11.47 \\
\hline 381.756 & 0.211 & 6.65 & 0.02225 & 0.02515 & -11.52 \\
\hline 381.944 & 0.211 & 6.66 & 0.02222 & 0.02517 & -11.71 \\
\hline 382.669 & 0.210 & 6.61 & 0.02219 & 0.02524 & -12.10 \\
\hline 381.762 & 0.107 & 3.36 & 0.02207 & 0.02515 & -12.23 \\
\hline 381.967 & 0.107 & 3.35 & 0.02202 & 0.02517 & -12.51 \\
\hline 382.203 & 0.107 & 3.36 & 0.02196 & 0.02519 & -12.83 \\
\hline 382.416 & 0.107 & 3.36 & 0.02198 & 0.02522 & -12.83 \\
\hline 382.689 & 0.108 & 3.37 & 0.02195 & 0.02524 & -13.05 \\
\hline 401.816 & 0.471 & 14.31 & 0.02485 & 0.02732 & -9.03 \\
\hline 401.513 & 0.473 & 14.41 & 0.02442 & 0.02729 & -10.50 \\
\hline 401.721 & 0.473 & 14.39 & 0.02436 & 0.02731 & -10.79 \\
\hline 401.921 & 0.473 & 14.39 & 0.02435 & 0.02733 & -10.90 \\
\hline 402.169 & 0.474 & 14.40 & 0.02443 & 0.02736 & -10.70 \\
\hline 402.388 & 0.473 & 14.37 & 0.02441 & 0.02738 & -10.85 \\
\hline 401.717 & 0.317 & 9.55 & 0.02460 & 0.02730 & -9.90 \\
\hline 401.904 & 0.317 & 9.54 & 0.02456 & 0.02732 & -10.12 \\
\hline
\end{tabular}


Table 10. Thermal conductivity for R507A (continued)

\begin{tabular}{|c|c|c|c|c|c|}
\hline $\begin{array}{c}\text { Temperature } \\
(\mathrm{K})\end{array}$ & $\begin{array}{l}\text { Pressure } \\
(\mathrm{MPa})\end{array}$ & $\begin{array}{c}\text { Density (calc) } \\
\left(\mathrm{kg} / \mathrm{m}^{3}\right)\end{array}$ & $\begin{array}{c}\lambda_{\exp } \\
(\mathrm{W} / \mathrm{m} \cdot \mathrm{K})\end{array}$ & $\begin{array}{c}\lambda_{\text {calc }} \\
(\mathrm{W} / \mathrm{m} \cdot \mathrm{K})\end{array}$ & $\begin{array}{l}\Delta \lambda \\
(\%)\end{array}$ \\
\hline 402.161 & 0.316 & 9.52 & 0.02453 & 0.02735 & -10.32 \\
\hline 402.386 & 0.316 & 9.52 & 0.02452 & 0.02738 & -10.44 \\
\hline 401.506 & 0.215 & 6.43 & 0.02456 & 0.02728 & -9.97 \\
\hline 401.722 & 0.214 & 6.41 & 0.02448 & 0.02730 & -10.34 \\
\hline 401.952 & 0.214 & 6.40 & 0.02441 & 0.02733 & -10.68 \\
\hline 402.141 & 0.214 & 6.41 & 0.02443 & 0.02735 & -10.67 \\
\hline 402.407 & 0.215 & 6.42 & 0.02445 & 0.02738 & -10.70 \\
\hline 401.523 & 0.109 & 3.24 & 0.02425 & 0.02728 & -11.11 \\
\hline 401.936 & 0.109 & 3.24 & 0.02415 & 0.02733 & -11.62 \\
\hline 402.171 & 0.109 & 3.25 & 0.02414 & 0.02735 & -11.74 \\
\hline 402.424 & 0.109 & 3.23 & 0.02413 & 0.02738 & -11.87 \\
\hline 300.010 & 0.294 & 12.26 & 0.01431 & 0.01688 & -15.24 \\
\hline 300.258 & 0.286 & 11.89 & 0.01429 & 0.01691 & -15.47 \\
\hline 300.526 & 0.279 & 11.55 & 0.01432 & 0.01693 & -15.42 \\
\hline 300.816 & 0.271 & 11.22 & 0.01433 & 0.01696 & -15.49 \\
\hline 301.127 & 0.263 & 10.86 & 0.01434 & 0.01699 & -15.58 \\
\hline 299.819 & 0.108 & 4.34 & 0.01406 & 0.01685 & -16.57 \\
\hline 300.326 & 0.107 & 4.33 & 0.01403 & 0.01690 & -16.98 \\
\hline 300.613 & 0.107 & 4.30 & 0.01404 & 0.01693 & -17.06 \\
\hline 300.921 & 0.106 & 4.25 & 0.01405 & 0.01696 & -17.14 \\
\hline 315.132 & 0.303 & 11.94 & 0.01565 & 0.01834 & -14.66 \\
\hline 315.354 & 0.303 & 11.90 & 0.01562 & 0.01836 & -14.92 \\
\hline 315.583 & 0.301 & 11.83 & 0.01563 & 0.01838 & -14.97 \\
\hline 316.134 & 0.300 & 11.77 & 0.01569 & 0.01844 & -14.89 \\
\hline 315.152 & 0.209 & 8.10 & 0.01562 & 0.01833 & -14.80 \\
\hline 315.355 & 0.208 & 8.06 & 0.01557 & 0.01835 & -15.17 \\
\hline 315.878 & 0.208 & 8.04 & 0.01555 & 0.01840 & -15.51 \\
\hline 316.160 & 0.208 & 8.03 & 0.01555 & 0.01843 & -15.64 \\
\hline 314.911 & 0.108 & 4.13 & 0.01553 & 0.01831 & -15.17 \\
\hline 315.648 & 0.108 & 4.12 & 0.01544 & 0.01838 & -15.99 \\
\hline 315.905 & 0.108 & 4.12 & 0.01544 & 0.01840 & -16.10 \\
\hline 330.582 & 0.306 & 11.41 & 0.01712 & 0.01986 & -13.80 \\
\hline 330.808 & 0.306 & 11.41 & 0.01711 & 0.01988 & -13.95 \\
\hline 331.068 & 0.305 & 11.37 & 0.01710 & 0.01991 & -14.11 \\
\hline 330.371 & 0.206 & 7.58 & 0.01708 & 0.01983 & -13.89 \\
\hline 330.578 & 0.205 & 7.55 & 0.01703 & 0.01986 & -14.23 \\
\hline 330.846 & 0.205 & 7.53 & 0.01701 & 0.01988 & -14.45 \\
\hline 331.083 & 0.205 & 7.54 & 0.01702 & 0.01991 & -14.50 \\
\hline 330.181 & 0.107 & 3.90 & 0.01695 & 0.01981 & -14.45 \\
\hline 330.863 & 0.107 & 3.88 & 0.01685 & 0.01988 & -15.25 \\
\hline 331.119 & 0.107 & 3.90 & 0.01687 & 0.01991 & -15.26 \\
\hline
\end{tabular}


Table 11. Thermal conductivity for R507A measured by the transient hot-wire technique; data are listed in the order of measurement.

\begin{tabular}{|c|c|c|c|c|c|}
\hline $\begin{array}{c}\text { Temperature } \\
\text { (K) }\end{array}$ & $\begin{array}{c}\text { Pressure } \\
(\mathrm{MPa})\end{array}$ & $\begin{array}{c}\text { Density (calc) } \\
\left(\mathrm{kg} / \mathrm{m}^{3}\right)\end{array}$ & $\begin{array}{c}\lambda_{\exp } \\
(\mathrm{W} / \mathrm{m} \cdot \mathrm{K})\end{array}$ & $\begin{array}{c}\lambda_{\text {calc }} \\
(\mathrm{W} / \mathrm{m} \cdot \mathrm{K})\end{array}$ & $\begin{array}{l}\Delta \lambda \\
(\%)\end{array}$ \\
\hline 301.498 & 32.50 & 1212.4 & 0.07949 & 0.08290 & -4.11 \\
\hline 301.673 & 32.48 & 1211.9 & 0.07916 & 0.08285 & -4.45 \\
\hline 301.869 & 32.47 & 1211.4 & 0.07906 & 0.08279 & -4.50 \\
\hline 302.065 & 32.46 & 1210.9 & 0.07933 & 0.08273 & -4.11 \\
\hline 302.281 & 32.46 & 1210.4 & 0.07911 & 0.08268 & -4.31 \\
\hline 301.517 & 25.47 & 1187.4 & 0.07549 & 0.07917 & -4.65 \\
\hline 301.727 & 25.47 & 1186.8 & 0.07581 & 0.07912 & -4.18 \\
\hline 301.926 & 25.46 & 1186.3 & 0.07577 & 0.07906 & -4.16 \\
\hline 302.132 & 25.47 & 1185.8 & 0.07559 & 0.07901 & -4.33 \\
\hline 302.364 & 25.48 & 1185.2 & 0.07566 & 0.07895 & -4.17 \\
\hline 301.587 & 17.86 & 1154.6 & 0.07140 & 0.07458 & -4.26 \\
\hline 301.789 & 17.86 & 1154.0 & 0.07171 & 0.07453 & -3.78 \\
\hline 302.019 & 17.86 & 1153.3 & 0.07190 & 0.07446 & -3.44 \\
\hline 302.232 & 17.86 & 1152.7 & 0.07145 & 0.07441 & -3.97 \\
\hline 302.460 & 17.87 & 1152.0 & 0.07154 & 0.07435 & -3.77 \\
\hline 301.636 & 12.22 & 1124.2 & 0.06919 & 0.07117 & -2.79 \\
\hline 301.873 & 12.21 & 1123.4 & 0.06906 & 0.07110 & -2.87 \\
\hline 302.092 & 12.21 & 1122.6 & 0.06919 & 0.07104 & -2.60 \\
\hline 302.331 & 12.22 & 1121.9 & 0.06846 & 0.07097 & -3.53 \\
\hline 302.564 & 12.22 & 1121.1 & 0.06941 & 0.07090 & -2.10 \\
\hline 301.297 & 12.24 & 1125.5 & 0.06924 & 0.07128 & -2.87 \\
\hline 301.305 & 12.24 & 1125.4 & 0.06900 & 0.07128 & -3.20 \\
\hline 301.495 & 12.24 & 1124.8 & 0.06922 & 0.07123 & -2.82 \\
\hline 301.494 & 12.24 & 1124.8 & 0.07000 & 0.07123 & -1.72 \\
\hline 301.695 & 12.24 & 1124.1 & 0.06980 & 0.07117 & -1.92 \\
\hline 301.699 & 12.23 & 1124.1 & 0.06941 & 0.07116 & -2.46 \\
\hline 301.916 & 12.23 & 1123.4 & 0.06867 & 0.07110 & -3.42 \\
\hline 301.904 & 12.23 & 1123.4 & 0.07097 & 0.07110 & -0.19 \\
\hline 302.121 & 12.23 & 1122.6 & 0.07019 & 0.07104 & -1.19 \\
\hline 302.128 & 12.23 & 1122.6 & 0.06906 & 0.07104 & -2.78 \\
\hline 301.339 & 7.95 & 1096.8 & 0.06879 & 0.06834 & 0.66 \\
\hline 301.348 & 7.95 & 1096.7 & 0.06841 & 0.06834 & 0.10 \\
\hline 301.541 & 7.95 & 1096.0 & 0.06849 & 0.06828 & 0.31 \\
\hline 301.549 & 7.95 & 1095.9 & 0.06782 & 0.06827 & -0.67 \\
\hline 301.756 & 7.95 & 1095.1 & 0.06815 & 0.06821 & -0.09 \\
\hline 301.751 & 7.96 & 1095.2 & 0.06881 & 0.06821 & 0.88 \\
\hline 301.967 & 7.96 & 1094.4 & 0.06798 & 0.06814 & -0.24 \\
\hline 301.969 & 7.96 & 1094.4 & 0.06858 & 0.06814 & 0.64 \\
\hline 302.201 & 7.96 & 1093.5 & 0.06792 & 0.06807 & -0.22 \\
\hline 302.195 & 7.96 & 1093.5 & 0.06930 & 0.06807 & 1.80 \\
\hline 301.025 & 4.25 & 1066.4 & 0.06567 & 0.06550 & 0.26 \\
\hline 301.028 & 4.25 & 1066.4 & 0.06738 & 0.06550 & 2.87 \\
\hline 301.210 & 4.25 & 1065.6 & 0.06597 & 0.06544 & 0.81 \\
\hline 301.203 & 4.25 & 1065.6 & 0.06660 & 0.06544 & 1.78 \\
\hline 301.399 & 4.25 & 1064.7 & 0.06727 & 0.06537 & 2.91 \\
\hline 301.414 & 4.25 & 1064.7 & 0.06530 & 0.06537 & -0.10 \\
\hline 301.601 & 4.25 & 1063.8 & 0.06742 & 0.06530 & 3.25 \\
\hline
\end{tabular}


Table 11. Thermal conductivity for R507A (continued)

\begin{tabular}{|c|c|c|c|c|c|}
\hline $\begin{array}{l}\text { Temperature } \\
\text { (K) }\end{array}$ & $\begin{array}{c}\text { Pressure } \\
(\mathrm{MPa})\end{array}$ & $\begin{array}{c}\text { Density (calc) } \\
\left(\mathrm{kg} / \mathrm{m}^{3}\right)\end{array}$ & $\begin{array}{c}\lambda_{\exp } \\
(\mathrm{W} / \mathrm{m} \cdot \mathrm{K})\end{array}$ & $\begin{array}{c}\lambda_{\text {calc }} \\
(\mathrm{W} / \mathrm{m} \cdot \mathrm{K})\end{array}$ & $\begin{array}{l}\Delta \lambda \\
(\%)\end{array}$ \\
\hline 301.818 & 4.25 & 1062.8 & 0.06784 & 0.06522 & 4.01 \\
\hline 301.829 & 4.25 & 1062.8 & 0.06659 & 0.06522 & 2.10 \\
\hline 301.056 & 1.54 & 1035.4 & 0.06581 & 0.06292 & 4.60 \\
\hline 301.257 & 1.54 & 1034.3 & 0.06475 & 0.06284 & 3.05 \\
\hline 301.433 & 1.53 & 1033.3 & 0.06627 & 0.06276 & 5.59 \\
\hline 301.652 & 1.54 & 1032.1 & 0.06616 & 0.06268 & 5.56 \\
\hline 301.860 & 1.53 & 1030.9 & 0.06555 & 0.06259 & 4.73 \\
\hline 361.909 & 26.29 & 1028.3 & 0.06562 & 0.06661 & -1.48 \\
\hline 362.117 & 26.30 & 1027.8 & 0.06511 & 0.06658 & -2.20 \\
\hline 362.345 & 26.30 & 1027.2 & 0.06532 & 0.06654 & -1.84 \\
\hline 362.574 & 26.29 & 1026.5 & 0.06556 & 0.06650 & -1.42 \\
\hline 362.821 & 26.28 & 1025.7 & 0.06537 & 0.06646 & -1.63 \\
\hline 361.946 & 21.67 & 994.3 & 0.06200 & 0.06376 & -2.76 \\
\hline 362.165 & 21.68 & 993.7 & 0.06214 & 0.06373 & -2.49 \\
\hline 362.401 & 21.68 & 993.0 & 0.06227 & 0.06369 & -2.23 \\
\hline 362.650 & 21.68 & 992.2 & 0.06198 & 0.06365 & -2.62 \\
\hline 362.903 & 21.66 & 991.3 & 0.06221 & 0.06360 & -2.18 \\
\hline 361.811 & 16.13 & 940.8 & 0.05768 & 0.05976 & -3.48 \\
\hline 362.027 & 16.14 & 940.1 & 0.05768 & 0.05972 & -3.42 \\
\hline 362.257 & 16.14 & 939.3 & 0.05788 & 0.05969 & -3.03 \\
\hline 362.509 & 16.14 & 938.3 & 0.05768 & 0.05964 & -3.29 \\
\hline 362.766 & 16.13 & 937.2 & 0.05759 & 0.05959 & -3.35 \\
\hline 361.855 & 12.63 & 892.3 & 0.05415 & 0.05665 & -4.41 \\
\hline 362.093 & 12.63 & 891.2 & 0.05411 & 0.05660 & -4.41 \\
\hline 362.348 & 12.64 & 890.2 & 0.05418 & 0.05656 & -4.21 \\
\hline 362.611 & 12.64 & 889.1 & 0.05424 & 0.05651 & -4.02 \\
\hline 362.895 & 12.65 & 887.8 & 0.05417 & 0.05646 & -4.06 \\
\hline 361.727 & 9.93 & 838.9 & 0.05097 & 0.05371 & -5.11 \\
\hline 361.955 & 9.92 & 837.5 & 0.05122 & 0.05366 & -4.54 \\
\hline 362.191 & 9.92 & 836.1 & 0.05063 & 0.05360 & -5.55 \\
\hline 362.452 & 9.93 & 834.7 & 0.05073 & 0.05355 & -5.26 \\
\hline 362.727 & 9.93 & 833.2 & 0.05080 & 0.05349 & -5.03 \\
\hline 361.784 & 8.21 & 787.2 & 0.04848 & 0.05135 & -5.59 \\
\hline 362.023 & 8.21 & 785.4 & 0.04838 & 0.05129 & -5.67 \\
\hline 362.275 & 8.21 & 783.5 & 0.04824 & 0.05123 & -5.83 \\
\hline 362.538 & 8.21 & 781.7 & 0.04833 & 0.05116 & -5.54 \\
\hline 362.822 & 8.22 & 779.6 & 0.04815 & 0.05110 & -5.77 \\
\hline 361.816 & 7.03 & 732.6 & 0.04610 & 0.04938 & -6.64 \\
\hline 362.064 & 7.03 & 730.2 & 0.04627 & 0.04931 & -6.16 \\
\hline 362.334 & 7.02 & 727.5 & 0.04593 & 0.04923 & -6.70 \\
\hline 362.620 & 7.02 & 724.6 & 0.04583 & 0.04915 & -6.75 \\
\hline 362.899 & 7.03 & 721.9 & 0.04587 & 0.04907 & -6.53 \\
\hline 361.625 & 6.29 & 681.7 & 0.04436 & 0.04806 & -7.69 \\
\hline 361.853 & 6.28 & 678.6 & 0.04458 & 0.04799 & -7.10 \\
\hline 362.109 & 6.28 & 675.2 & 0.04410 & 0.04791 & -7.96 \\
\hline 362.391 & 6.29 & 671.6 & 0.04416 & 0.04783 & -7.68 \\
\hline 362.673 & 6.29 & 668.0 & 0.04404 & 0.04776 & -7.78 \\
\hline
\end{tabular}


Table 11. Thermal conductivity for R507A (continued)

\begin{tabular}{|c|c|c|c|c|c|}
\hline $\begin{array}{l}\text { Temperature } \\
\text { (K) }\end{array}$ & $\begin{array}{c}\text { Pressure } \\
(\mathrm{MPa})\end{array}$ & $\begin{array}{c}\text { Density (calc) } \\
\left(\mathrm{kg} / \mathrm{m}^{3}\right)\end{array}$ & $\begin{array}{c}\lambda_{\exp } \\
(\mathrm{W} / \mathrm{m} \cdot \mathrm{K})\end{array}$ & $\begin{array}{c}\lambda_{\text {calc }} \\
(\mathrm{W} / \mathrm{m} \cdot \mathrm{K})\end{array}$ & $\begin{array}{l}\Delta \lambda \\
(\%)\end{array}$ \\
\hline 361.448 & 5.80 & 630.0 & 0.04286 & 0.04724 & -9.27 \\
\hline 361.653 & 5.80 & 626.6 & 0.04284 & 0.04718 & -9.20 \\
\hline 361.882 & 5.80 & 622.7 & 0.04303 & 0.04711 & -8.66 \\
\hline 362.122 & 5.80 & 618.5 & 0.04265 & 0.04704 & -9.33 \\
\hline 362.392 & 5.80 & 613.8 & 0.04276 & 0.04695 & -8.93 \\
\hline 361.427 & 5.44 & 567.4 & 0.04190 & 0.04659 & -10.07 \\
\hline 361.429 & 5.44 & 567.4 & 0.04187 & 0.04659 & -10.13 \\
\hline 361.646 & 5.44 & 562.5 & 0.04163 & 0.04650 & -10.47 \\
\hline 361.654 & 5.44 & 562.4 & 0.04161 & 0.04649 & -10.51 \\
\hline 361.883 & 5.44 & 557.3 & 0.04145 & 0.04639 & -10.65 \\
\hline 361.890 & 5.44 & 557.2 & 0.04164 & 0.04639 & -10.24 \\
\hline 362.140 & 5.44 & 551.6 & 0.04118 & 0.04627 & -11.01 \\
\hline 362.138 & 5.44 & 551.7 & 0.04126 & 0.04627 & -10.84 \\
\hline 362.402 & 5.44 & 545.9 & 0.04112 & 0.04614 & -10.89 \\
\hline 362.396 & 5.44 & 546.0 & 0.04120 & 0.04615 & -10.72 \\
\hline 361.312 & 5.24 & 518.7 & 0.04083 & 0.04592 & -11.09 \\
\hline 361.321 & 5.24 & 518.6 & 0.04088 & 0.04592 & -10.97 \\
\hline 361.515 & 5.24 & 513.8 & 0.04061 & 0.04578 & -11.29 \\
\hline 361.520 & 5.24 & 513.7 & 0.04044 & 0.04578 & -11.66 \\
\hline 361.733 & 5.24 & 508.5 & 0.04023 & 0.04562 & -11.81 \\
\hline 361.715 & 5.24 & 508.9 & 0.04036 & 0.04563 & -11.56 \\
\hline 361.957 & 5.24 & 503.0 & 0.04010 & 0.04545 & -11.76 \\
\hline 361.961 & 5.24 & 502.8 & 0.04013 & 0.04544 & -11.69 \\
\hline 362.193 & 5.24 & 497.2 & 0.03978 & 0.04526 & -12.10 \\
\hline 362.218 & 5.24 & 496.6 & 0.03969 & 0.04524 & -12.26 \\
\hline 361.326 & 5.06 & 465.0 & 0.03899 & 0.04453 & -12.44 \\
\hline 361.337 & 5.06 & 464.6 & 0.03935 & 0.04451 & -11.60 \\
\hline 361.528 & 5.06 & 460.0 & 0.03869 & 0.04431 & -12.68 \\
\hline 361.538 & 5.06 & 459.8 & 0.03875 & 0.04430 & -12.52 \\
\hline 361.766 & 5.06 & 454.5 & 0.03843 & 0.04405 & -12.77 \\
\hline 361.762 & 5.06 & 454.7 & 0.03844 & 0.04406 & -12.76 \\
\hline 362.004 & 5.06 & 449.2 & 0.03808 & 0.04380 & -13.06 \\
\hline 362.011 & 5.06 & 449.1 & 0.03821 & 0.04380 & -12.76 \\
\hline 362.265 & 5.06 & 443.6 & 0.03766 & 0.04352 & -13.47 \\
\hline 362.266 & 5.06 & 443.5 & 0.03776 & 0.04352 & -13.24 \\
\hline 361.390 & 4.91 & 416.8 & 0.03724 & 0.04255 & -12.49 \\
\hline 361.391 & 4.91 & 416.7 & 0.03721 & 0.04255 & -12.55 \\
\hline 361.610 & 4.91 & 412.4 & 0.03676 & 0.04229 & -13.08 \\
\hline 361.605 & 4.91 & 412.4 & 0.03670 & 0.04230 & -13.23 \\
\hline 361.834 & 4.91 & 407.9 & 0.03654 & 0.04203 & -13.06 \\
\hline 361.840 & 4.91 & 407.8 & 0.03633 & 0.04202 & -13.54 \\
\hline 362.100 & 4.91 & 403.0 & 0.03591 & 0.04172 & -13.93 \\
\hline 362.100 & 4.91 & 402.9 & 0.03598 & 0.04172 & -13.76 \\
\hline 362.377 & 4.91 & 397.9 & 0.03558 & 0.04141 & -14.08 \\
\hline 362.390 & 4.91 & 397.8 & 0.03556 & 0.04140 & -14.11 \\
\hline 361.508 & 4.76 & 370.4 & 0.03454 & 0.04006 & -13.77 \\
\hline 361.514 & 4.76 & 370.4 & 0.03452 & 0.04005 & -13.81 \\
\hline 361.728 & 4.76 & 367.1 & 0.03431 & 0.03982 & -13.85 \\
\hline
\end{tabular}


Table 11. Thermal conductivity for R507A (continued)

\begin{tabular}{|c|c|c|c|c|c|}
\hline $\begin{array}{l}\text { Temperature } \\
\text { (K) }\end{array}$ & $\begin{array}{c}\text { Pressure } \\
(\mathrm{MPa})\end{array}$ & $\begin{array}{c}\text { Density (calc) } \\
\left(\mathrm{kg} / \mathrm{m}^{3}\right)\end{array}$ & $\begin{array}{c}\lambda_{\exp } \\
(\mathrm{W} / \mathrm{m} \cdot \mathrm{K})\end{array}$ & $\begin{array}{c}\lambda_{\text {calc }} \\
(\mathrm{W} / \mathrm{m} \cdot \mathrm{K})\end{array}$ & $\begin{array}{l}\Delta \lambda \\
(\%)\end{array}$ \\
\hline 361.745 & 4.76 & 366.9 & 0.03432 & 0.03981 & -13.79 \\
\hline 361.999 & 4.76 & 363.1 & 0.03403 & 0.03955 & -13.95 \\
\hline 362.009 & 4.75 & 363.0 & 0.03400 & 0.03953 & -14.00 \\
\hline 362.297 & 4.75 & 358.9 & 0.03358 & 0.03925 & -14.44 \\
\hline 362.297 & 4.75 & 358.9 & 0.03351 & 0.03925 & -14.62 \\
\hline 362.599 & 4.75 & 354.8 & 0.03324 & 0.03896 & -14.69 \\
\hline 362.621 & 4.75 & 354.6 & 0.03321 & 0.03894 & -14.73 \\
\hline 361.649 & 4.58 & 327.2 & 0.03182 & 0.03736 & -14.83 \\
\hline 361.666 & 4.58 & 327.0 & 0.03208 & 0.03734 & -14.10 \\
\hline 361.923 & 4.58 & 324.3 & 0.03185 & 0.03714 & -14.25 \\
\hline 361.931 & 4.58 & 324.3 & 0.03184 & 0.03714 & -14.27 \\
\hline 362.231 & 4.58 & 321.3 & 0.03171 & 0.03692 & -14.10 \\
\hline 362.238 & 4.58 & 321.2 & 0.03159 & 0.03691 & -14.42 \\
\hline 362.563 & 4.58 & 318.0 & 0.03124 & 0.03668 & -14.83 \\
\hline 362.563 & 4.58 & 318.1 & 0.03121 & 0.03668 & -14.92 \\
\hline 362.907 & 4.58 & 314.8 & 0.03107 & 0.03645 & -14.75 \\
\hline 362.920 & 4.58 & 314.8 & 0.03110 & 0.03644 & -14.66 \\
\hline 361.877 & 4.36 & 283.7 & 0.02932 & 0.03449 & -14.99 \\
\hline 361.881 & 4.36 & 283.7 & 0.02940 & 0.03449 & -14.75 \\
\hline 362.185 & 4.36 & 281.5 & 0.02938 & 0.03433 & -14.42 \\
\hline 362.199 & 4.36 & 281.5 & 0.02942 & 0.03433 & -14.30 \\
\hline 362.534 & 4.36 & 279.3 & 0.02918 & 0.03417 & -14.61 \\
\hline 362.532 & 4.37 & 279.3 & 0.02914 & 0.03417 & -14.73 \\
\hline 362.909 & 4.37 & 276.9 & 0.02901 & 0.03400 & -14.69 \\
\hline 362.914 & 4.37 & 276.9 & 0.02906 & 0.03400 & -14.54 \\
\hline 363.306 & 4.37 & 274.5 & 0.02885 & 0.03384 & -14.74 \\
\hline 363.309 & 4.37 & 274.5 & 0.02885 & 0.03384 & -14.73 \\
\hline 361.844 & 4.02 & 234.4 & 0.02675 & 0.03131 & -14.56 \\
\hline 361.852 & 4.02 & 234.4 & 0.02670 & 0.03131 & -14.72 \\
\hline 362.188 & 4.02 & 233.0 & 0.02681 & 0.03123 & -14.15 \\
\hline 362.190 & 4.03 & 233.0 & 0.02684 & 0.03123 & -14.06 \\
\hline 362.558 & 4.03 & 231.6 & 0.02674 & 0.03115 & -14.15 \\
\hline 362.556 & 4.03 & 231.6 & 0.02671 & 0.03115 & -14.25 \\
\hline 362.956 & 4.03 & 230.1 & 0.02674 & 0.03106 & -13.91 \\
\hline 362.969 & 4.03 & 230.0 & 0.02666 & 0.03106 & -14.16 \\
\hline 363.408 & 4.03 & 228.3 & 0.02654 & 0.03096 & -14.29 \\
\hline 363.405 & 4.03 & 228.3 & 0.02652 & 0.03096 & -14.35 \\
\hline 362.095 & 3.62 & 189.3 & 0.02461 & 0.02868 & -14.19 \\
\hline 362.095 & 3.62 & 189.4 & 0.02458 & 0.02868 & -14.30 \\
\hline 362.487 & 3.62 & 188.5 & 0.02458 & 0.02865 & -14.22 \\
\hline 362.489 & 3.62 & 188.5 & 0.02458 & 0.02865 & -14.22 \\
\hline 362.913 & 3.62 & 187.5 & 0.02458 & 0.02863 & -14.13 \\
\hline 362.926 & 3.62 & 187.5 & 0.02454 & 0.02863 & -14.27 \\
\hline 363.388 & 3.62 & 186.5 & 0.02455 & 0.02860 & -14.15 \\
\hline 363.372 & 3.62 & 186.5 & 0.02460 & 0.02860 & -13.98 \\
\hline 363.878 & 3.62 & 185.3 & 0.02454 & 0.02856 & -14.09 \\
\hline 363.882 & 3.62 & 185.3 & 0.02461 & 0.02856 & -13.84 \\
\hline 362.400 & 3.03 & 140.4 & 0.02278 & 0.02632 & -13.46 \\
\hline
\end{tabular}


Table 11. Thermal conductivity for R507A (continued)

\begin{tabular}{|c|c|c|c|c|c|}
\hline $\begin{array}{l}\text { Temperature } \\
\text { (K) }\end{array}$ & $\begin{array}{l}\text { Pressure } \\
(\mathrm{MPa})\end{array}$ & $\begin{array}{c}\text { Density (calc) } \\
\left(\mathrm{kg} / \mathrm{m}^{3}\right)\end{array}$ & $\begin{array}{c}\lambda_{\exp } \\
(\mathrm{W} / \mathrm{m} \cdot \mathrm{K})\end{array}$ & $\begin{array}{c}\lambda_{\text {calc }} \\
(\mathrm{W} / \mathrm{m} \cdot \mathrm{K})\end{array}$ & $\begin{array}{l}\Delta \lambda \\
(\%)\end{array}$ \\
\hline 362.414 & 3.03 & 140.4 & 0.02275 & 0.02632 & -13.58 \\
\hline 362.850 & 3.03 & 139.9 & 0.02274 & 0.02634 & -13.66 \\
\hline 362.863 & 3.03 & 139.9 & 0.02280 & 0.02634 & -13.44 \\
\hline 363.344 & 3.02 & 139.3 & 0.02279 & 0.02635 & -13.52 \\
\hline 363.360 & 3.02 & 139.2 & 0.02274 & 0.02635 & -13.71 \\
\hline 363.891 & 3.02 & 138.6 & 0.02277 & 0.02637 & -13.65 \\
\hline 363.893 & 3.02 & 138.5 & 0.02279 & 0.02637 & -13.57 \\
\hline 364.465 & 3.02 & 137.9 & 0.02277 & 0.02639 & -13.72 \\
\hline 364.476 & 3.02 & 137.9 & 0.02279 & 0.02639 & -13.64 \\
\hline 362.469 & 3.02 & 140.1 & 0.02278 & 0.02631 & -13.43 \\
\hline 362.470 & 3.02 & 140.1 & 0.02269 & 0.02631 & -13.77 \\
\hline 362.924 & 3.02 & 139.5 & 0.02272 & 0.02633 & -13.70 \\
\hline 362.920 & 3.02 & 139.5 & 0.02271 & 0.02633 & -13.74 \\
\hline 363.421 & 3.02 & 139.0 & 0.02277 & 0.02635 & -13.58 \\
\hline 363.413 & 3.02 & 139.0 & 0.02277 & 0.02635 & -13.58 \\
\hline 363.943 & 3.02 & 138.5 & 0.02276 & 0.02637 & -13.69 \\
\hline 363.943 & 3.02 & 138.5 & 0.02277 & 0.02637 & -13.65 \\
\hline 364.524 & 3.02 & 137.9 & 0.02281 & 0.02639 & -13.58 \\
\hline 364.518 & 3.02 & 137.9 & 0.02278 & 0.02639 & -13.69 \\
\hline 362.782 & 2.22 & 91.4 & 0.02111 & 0.02457 & -14.09 \\
\hline 362.799 & 2.22 & 91.4 & 0.02117 & 0.02457 & -13.85 \\
\hline 363.319 & 2.22 & 91.1 & 0.02120 & 0.02462 & -13.89 \\
\hline 363.319 & 2.23 & 91.2 & 0.02118 & 0.02462 & -13.97 \\
\hline 363.886 & 2.23 & 90.9 & 0.02124 & 0.02436 & -12.80 \\
\hline 363.895 & 2.23 & 90.9 & 0.02125 & 0.02436 & -12.77 \\
\hline 364.514 & 2.23 & 90.6 & 0.02128 & 0.02441 & -12.84 \\
\hline 364.518 & 2.23 & 90.5 & 0.02130 & 0.02441 & -12.75 \\
\hline 365.186 & 2.22 & 90.1 & 0.02129 & 0.02447 & -13.00 \\
\hline 365.183 & 2.22 & 90.0 & 0.02129 & 0.02447 & -12.99 \\
\hline 362.216 & 1.17 & 42.7 & 0.02002 & 0.02323 & -13.80 \\
\hline 362.715 & 1.15 & 41.9 & 0.02002 & 0.02327 & -13.96 \\
\hline 363.267 & 1.16 & 42.0 & 0.02018 & 0.02333 & -13.49 \\
\hline 363.895 & 1.16 & 42.2 & 0.02020 & 0.02340 & -13.66 \\
\hline 364.549 & 1.17 & 42.4 & 0.02023 & 0.02347 & -13.79 \\
\hline 362.398 & 0.64 & 22.1 & 0.01973 & 0.02312 & -14.67 \\
\hline 362.850 & 0.64 & 22.1 & 0.01978 & 0.02317 & -14.63 \\
\hline 363.332 & 0.64 & 22.1 & 0.01992 & 0.02322 & -14.21 \\
\hline 363.859 & 0.64 & 22.0 & 0.01993 & 0.02328 & -14.37 \\
\hline 364.419 & 0.64 & 21.9 & 0.01996 & 0.02333 & -14.46 \\
\hline 362.624 & 0.30 & 10.0 & 0.01982 & 0.02313 & -14.31 \\
\hline 363.110 & 0.30 & 9.9 & 0.01987 & 0.02318 & -14.28 \\
\hline 363.645 & 0.30 & 9.9 & 0.01990 & 0.02324 & -14.35 \\
\hline 364.225 & 0.30 & 9.9 & 0.01993 & 0.02330 & -14.45 \\
\hline 364.837 & 0.30 & 9.9 & 0.01997 & 0.02336 & -14.51 \\
\hline 362.494 & 0.20 & 6.8 & 0.01982 & 0.02311 & -14.25 \\
\hline 362.854 & 0.20 & 6.8 & 0.01983 & 0.02315 & -14.34 \\
\hline 363.240 & 0.21 & 6.8 & 0.01991 & 0.02319 & -14.15 \\
\hline 363.651 & 0.21 & 6.8 & 0.01989 & 0.02323 & -14.39 \\
\hline
\end{tabular}


Table 11. Thermal conductivity for R507A (continued)

\begin{tabular}{|c|c|c|c|c|c|}
\hline $\begin{array}{l}\text { Temperature } \\
\text { (K) }\end{array}$ & $\begin{array}{c}\text { Pressure } \\
(\mathrm{MPa})\end{array}$ & $\begin{array}{c}\text { Density (calc) } \\
\left(\mathrm{kg} / \mathrm{m}^{3}\right)\end{array}$ & $\begin{array}{c}\lambda_{\exp } \\
(\mathrm{W} / \mathrm{m} \cdot \mathrm{K})\end{array}$ & $\begin{array}{c}\lambda_{\text {calc }} \\
(\mathrm{W} / \mathrm{m} \cdot \mathrm{K})\end{array}$ & $\begin{array}{l}\Delta \lambda \\
(\%)\end{array}$ \\
\hline $\begin{array}{l}364.086 \\
362.652 \\
363.035 \\
363.447 \\
363.881 \\
364.341\end{array}$ & $\begin{array}{l}0.21 \\
0.11 \\
0.11 \\
0.11 \\
0.11 \\
0.11\end{array}$ & $\begin{array}{l}6.8 \\
3.5 \\
3.5 \\
3.5 \\
3.5 \\
3.5\end{array}$ & $\begin{array}{l}0.01993 \\
0.01993 \\
0.01991 \\
0.01994 \\
0.01994 \\
0.02001\end{array}$ & $\begin{array}{l}0.02328 \\
0.02313 \\
0.02317 \\
0.02321 \\
0.02326 \\
0.02330\end{array}$ & $\begin{array}{l}-14.39 \\
-13.83 \\
-14.06 \\
-14.09 \\
-14.26 \\
-14.14\end{array}$ \\
\hline 316810 & & & & & \\
\hline 317.015 & 37.79 & 1193.4 & 0.07963 & 0.08175 & -2.59 \\
\hline 317.214 & 37.80 & 1193.0 & 0.07955 & 0.08171 & -2.64 \\
\hline 317.429 & 37.81 & 1192.6 & 0.07945 & 0.08166 & -2.71 \\
\hline 317.633 & 37.81 & 1192.1 & 0.07946 & 0.08161 & -2.64 \\
\hline 316.868 & 28.56 & 1159.9 & 0.07498 & 0.07696 & -2.57 \\
\hline 317.079 & 28.56 & 1159.3 & 0.07499 & 0.07691 & -2.50 \\
\hline 317.284 & 28.58 & 1158.9 & 0.07491 & 0.07687 & -2.55 \\
\hline 317.511 & 28.59 & 1158.3 & 0.07477 & 0.07682 & -2.67 \\
\hline 317.743 & 28.59 & 1157.8 & 0.07482 & 0.07677 & -2.54 \\
\hline 316.736 & 21.86 & 1130.1 & 0.07099 & 0.07295 & -2.68 \\
\hline 316.947 & 21.87 & 1129.5 & 0.07107 & 0.07290 & -2.51 \\
\hline 317.138 & 21.87 & 1129.0 & 0.07118 & 0.07286 & -2.30 \\
\hline 317.387 & 21.85 & 1128.2 & 0.07103 & 0.07278 & -2.41 \\
\hline 317.614 & 21.84 & 1127.5 & 0.07087 & 0.07273 & -2.55 \\
\hline 316.804 & 16.55 & 1100.7 & 0.06749 & 0.06978 & -3.28 \\
\hline 317.023 & 16.56 & 1100.1 & 0.06754 & 0.06973 & -3.14 \\
\hline 317.247 & 16.57 & 1099.4 & 0.06746 & 0.06968 & -3.19 \\
\hline 317.472 & 16.58 & 1098.7 & 0.06742 & 0.06963 & -3.17 \\
\hline 317.715 & 16.58 & 1098.0 & 0.06740 & 0.06957 & -3.12 \\
\hline 316.857 & 12.29 & 1071.7 & 0.06474 & 0.06693 & -3.27 \\
\hline 317.091 & 12.30 & 1070.8 & 0.06483 & 0.06687 & -3.04 \\
\hline 317.327 & 12.30 & 1070.0 & 0.06488 & 0.06680 & -2.88 \\
\hline 317.575 & 12.30 & 1069.1 & 0.06467 & 0.06674 & -3.10 \\
\hline 317.829 & 12.29 & 1068.1 & 0.06443 & 0.06666 & -3.35 \\
\hline 316.740 & 8.52 & 1039.7 & 0.06238 & 0.06406 & -2.63 \\
\hline 316.953 & 8.50 & 1038.6 & 0.06246 & 0.06399 & -2.39 \\
\hline 317.194 & 8.50 & 1037.6 & 0.06149 & 0.06391 & -3.79 \\
\hline 317.439 & 8.50 & 1036.5 & 0.06135 & 0.06384 & -3.90 \\
\hline 317.682 & 8.50 & 1035.5 & 0.06265 & 0.06377 & -1.76 \\
\hline 316.800 & 5.75 & 1008.3 & 0.05979 & 0.06154 & -2.85 \\
\hline 317.026 & 5.74 & 1007.1 & 0.06009 & 0.06147 & -2.24 \\
\hline 317.271 & 5.74 & 1005.9 & 0.05899 & 0.06138 & -3.90 \\
\hline 317.520 & 5.74 & 1004.6 & 0.05908 & 0.06130 & -3.63 \\
\hline 317.785 & 5.75 & 1003.4 & 0.05899 & 0.06123 & -3.65 \\
\hline 316.398 & 3.54 & 977.4 & 0.05886 & 0.05930 & -0.75 \\
\hline 316.630 & 3.54 & 976.0 & 0.05811 & 0.05921 & -1.87 \\
\hline 316.848 & 3.54 & 974.6 & 0.05809 & 0.05913 & -1.76 \\
\hline 317.106 & 3.54 & 973.0 & 0.05773 & 0.05904 & -2.21 \\
\hline 317.341 & 3.54 & 971.5 & 0.05741 & 0.05895 & -2.61 \\
\hline 316.102 & 2.19 & 951.7 & 0.05636 & 0.05765 & -2.25 \\
\hline
\end{tabular}


Table 11. Thermal conductivity for R507A (continued)

\begin{tabular}{|c|c|c|c|c|c|}
\hline $\begin{array}{c}\text { Temperature } \\
\text { (K) }\end{array}$ & $\begin{array}{c}\text { Pressure } \\
(\mathrm{MPa})\end{array}$ & $\begin{array}{c}\text { Density (calc) } \\
\left(\mathrm{kg} / \mathrm{m}^{3}\right)\end{array}$ & $\begin{array}{c}\lambda_{\exp } \\
(\mathrm{W} / \mathrm{m} \cdot \mathrm{K})\end{array}$ & $\begin{array}{c}\lambda_{\text {calc }} \\
(\mathrm{W} / \mathrm{m} \cdot \mathrm{K})\end{array}$ & $\begin{array}{l}\Delta \lambda \\
(\%)\end{array}$ \\
\hline 316.108 & 2.19 & 951.7 & 0.05734 & 0.05765 & -0.54 \\
\hline 316.298 & 2.19 & 950.3 & 0.05668 & 0.05758 & -1.56 \\
\hline 316.295 & 2.19 & 950.4 & 0.05627 & 0.05758 & -2.27 \\
\hline 316.494 & 2.19 & 948.9 & 0.05612 & 0.05750 & -2.40 \\
\hline 316.498 & 2.19 & 948.9 & 0.05610 & 0.05750 & -2.43 \\
\hline 316.706 & 2.19 & 947.3 & 0.05681 & 0.05741 & -1.04 \\
\hline 316.697 & 2.18 & 947.2 & 0.05725 & 0.05740 & -0.27 \\
\hline 316.936 & 2.18 & 945.4 & 0.05743 & 0.05730 & 0.22 \\
\hline 316.941 & 2.18 & 945.3 & 0.05570 & 0.05730 & -2.79 \\
\hline 381.305 & 23.15 & 947.7 & 0.06202 & 0.06195 & 0.12 \\
\hline 381.496 & 23.16 & 947.2 & 0.06205 & 0.06193 & 0.20 \\
\hline 381.678 & 23.15 & 946.6 & 0.06212 & 0.06190 & 0.36 \\
\hline 381.876 & 23.15 & 945.9 & 0.06178 & 0.06187 & -0.15 \\
\hline 382.085 & 23.15 & 945.4 & 0.06102 & 0.06185 & -1.34 \\
\hline 381.469 & 18.21 & 893.7 & 0.05657 & 0.05834 & -3.03 \\
\hline 381.650 & 18.22 & 893.2 & 0.05663 & 0.05832 & -2.89 \\
\hline 381.844 & 18.22 & 892.5 & 0.05630 & 0.05829 & -3.42 \\
\hline 382.050 & 18.22 & 891.8 & 0.05637 & 0.05826 & -3.25 \\
\hline 382.271 & 18.21 & 890.9 & 0.05647 & 0.05823 & -3.02 \\
\hline 381.624 & 14.70 & 840.1 & 0.05309 & 0.05525 & -3.90 \\
\hline 381.823 & 14.71 & 839.3 & 0.05311 & 0.05522 & -3.82 \\
\hline 382.035 & 14.71 & 838.4 & 0.05289 & 0.05519 & -4.17 \\
\hline 382.247 & 14.72 & 837.6 & 0.05310 & 0.05516 & -3.74 \\
\hline 382.458 & 14.72 & 836.7 & 0.05305 & 0.05513 & -3.78 \\
\hline 381.553 & 12.38 & 791.7 & 0.05014 & 0.05281 & -5.06 \\
\hline 381.750 & 12.38 & 790.7 & 0.05040 & 0.05278 & -4.51 \\
\hline 381.950 & 12.39 & 789.7 & 0.05040 & 0.05275 & -4.45 \\
\hline 382.149 & 12.39 & 788.8 & 0.04997 & 0.05272 & -5.22 \\
\hline 382.371 & 12.39 & 787.7 & 0.05012 & 0.05269 & -4.87 \\
\hline 381.650 & 10.58 & 738.6 & 0.04758 & 0.05051 & -5.81 \\
\hline 381.843 & 10.58 & 737.4 & 0.04777 & 0.05048 & -5.37 \\
\hline 382.044 & 10.58 & 736.2 & 0.04746 & 0.05044 & -5.92 \\
\hline 382.257 & 10.58 & 735.0 & 0.04744 & 0.05041 & -5.89 \\
\hline 382.482 & 10.59 & 733.7 & 0.04750 & 0.05037 & -5.70 \\
\hline 381.716 & 9.30 & 686.0 & 0.04551 & 0.04858 & -6.32 \\
\hline 381.920 & 9.31 & 684.6 & 0.04554 & 0.04854 & -6.18 \\
\hline 382.129 & 9.31 & 683.1 & 0.04540 & 0.04850 & -6.40 \\
\hline 382.345 & 9.31 & 681.6 & 0.04536 & 0.04846 & -6.40 \\
\hline 382.577 & 9.31 & 679.9 & 0.04537 & 0.04842 & -6.30 \\
\hline 381.633 & 8.40 & 635.5 & 0.04337 & 0.04700 & -7.73 \\
\hline 381.836 & 8.40 & 633.8 & 0.04357 & 0.04696 & -7.22 \\
\hline 382.055 & 8.40 & 632.0 & 0.04336 & 0.04692 & -7.58 \\
\hline 382.272 & 8.40 & 630.2 & 0.04321 & 0.04687 & -7.81 \\
\hline 382.511 & 8.40 & 628.2 & 0.04335 & 0.04682 & -7.41 \\
\hline 381.700 & 7.70 & 581.4 & 0.04172 & 0.04555 & -8.40 \\
\hline 381.904 & 7.70 & 579.6 & 0.04183 & 0.04550 & -8.06 \\
\hline 382.121 & 7.70 & 577.5 & 0.04174 & 0.04545 & -8.15 \\
\hline
\end{tabular}


Table 11. Thermal conductivity for R507A (continued)

\begin{tabular}{|c|c|c|c|c|c|}
\hline $\begin{array}{l}\text { Temperature } \\
\text { (K) }\end{array}$ & $\begin{array}{l}\text { Pressure } \\
(\mathrm{MPa})\end{array}$ & $\begin{array}{c}\text { Density (calc) } \\
\left(\mathrm{kg} / \mathrm{m}^{3}\right)\end{array}$ & $\begin{array}{c}\lambda_{\exp } \\
(\mathrm{W} / \mathrm{m} \cdot \mathrm{K})\end{array}$ & $\begin{array}{c}\lambda_{\text {calc }} \\
(\mathrm{W} / \mathrm{m} \cdot \mathrm{K})\end{array}$ & $\begin{array}{l}\Delta \lambda \\
(\%)\end{array}$ \\
\hline 382.357 & 7.71 & 575.4 & 0.04145 & 0.04539 & -8.68 \\
\hline 382.586 & 7.71 & 573.2 & 0.04156 & 0.04533 & -8.33 \\
\hline 381.591 & 7.19 & 532.3 & 0.03994 & 0.04427 & -9.78 \\
\hline 381.781 & 7.19 & 530.3 & 0.03982 & 0.04421 & -9.93 \\
\hline 382.002 & 7.19 & 527.9 & 0.03982 & 0.04414 & -9.80 \\
\hline 382.228 & 7.19 & 525.6 & 0.03988 & 0.04408 & -9.52 \\
\hline 382.454 & 7.19 & 523.3 & 0.03968 & 0.04401 & -9.84 \\
\hline 381.467 & 6.78 & 485.2 & 0.03837 & 0.04293 & -10.61 \\
\hline 381.665 & 6.78 & 483.1 & 0.03844 & 0.04286 & -10.31 \\
\hline 381.875 & 6.78 & 481.0 & 0.03836 & 0.04279 & -10.35 \\
\hline 382.085 & 6.78 & 478.9 & 0.03827 & 0.04271 & -10.40 \\
\hline 382.315 & 6.78 & 476.6 & 0.03793 & 0.04263 & -11.03 \\
\hline 381.525 & 6.78 & 484.7 & 0.03831 & 0.04291 & -10.72 \\
\hline 381.715 & 6.78 & 482.9 & 0.03839 & 0.04285 & -10.40 \\
\hline 381.901 & 6.78 & 481.0 & 0.03847 & 0.04279 & -10.09 \\
\hline 382.140 & 6.78 & 478.6 & 0.03824 & 0.04270 & -10.45 \\
\hline 382.369 & 6.78 & 476.2 & 0.03793 & 0.04262 & -11.01 \\
\hline 381.569 & 6.36 & 429.3 & 0.03647 & 0.04101 & -11.08 \\
\hline 381.789 & 6.36 & 427.4 & 0.03644 & 0.04093 & -10.98 \\
\hline 382.006 & 6.36 & 425.4 & 0.03624 & 0.04085 & -11.29 \\
\hline 382.237 & 6.36 & 423.3 & 0.03599 & 0.04077 & -11.72 \\
\hline 382.492 & 6.36 & 421.0 & 0.03586 & 0.04067 & -11.83 \\
\hline 381.462 & 6.01 & 383.5 & 0.03470 & 0.03916 & -11.38 \\
\hline 381.675 & 6.01 & 381.9 & 0.03452 & 0.03908 & -11.68 \\
\hline 381.890 & 6.01 & 380.3 & 0.03463 & 0.03901 & -11.23 \\
\hline 382.130 & 6.01 & 378.4 & 0.03433 & 0.03892 & -11.80 \\
\hline 382.381 & 6.01 & 376.4 & 0.03406 & 0.03884 & -12.30 \\
\hline 381.577 & 5.65 & 337.5 & 0.03249 & 0.03707 & -12.34 \\
\hline 381.796 & 5.65 & 336.3 & 0.03258 & 0.03701 & -11.96 \\
\hline 382.034 & 5.65 & 334.9 & 0.03254 & 0.03694 & -11.92 \\
\hline 382.294 & 5.65 & 333.5 & 0.03232 & 0.03688 & -12.35 \\
\hline 382.573 & 5.65 & 331.8 & 0.03202 & 0.03680 & -12.99 \\
\hline 381.711 & 5.24 & 290.3 & 0.03034 & 0.03477 & -12.75 \\
\hline 381.975 & 5.24 & 289.2 & 0.03037 & 0.03472 & -12.54 \\
\hline 382.227 & 5.25 & 288.1 & 0.03031 & 0.03468 & -12.60 \\
\hline 382.517 & 5.24 & 286.8 & 0.03017 & 0.03462 & -12.86 \\
\hline 382.832 & 5.24 & 285.4 & 0.03013 & 0.03456 & -12.83 \\
\hline 381.879 & 4.76 & 242.2 & 0.02837 & 0.03242 & -12.49 \\
\hline 382.157 & 4.76 & 241.4 & 0.02818 & 0.03239 & -13.01 \\
\hline 382.468 & 4.76 & 240.4 & 0.02815 & 0.03236 & -13.02 \\
\hline 382.794 & 4.76 & 239.4 & 0.02813 & 0.03233 & -13.00 \\
\hline 383.125 & 4.76 & 238.4 & 0.02810 & 0.03231 & -13.02 \\
\hline 382.105 & 4.15 & 192.3 & 0.02623 & 0.03013 & -12.94 \\
\hline 382.405 & 4.15 & 191.7 & 0.02621 & 0.03013 & -13.01 \\
\hline 382.751 & 4.15 & 191.1 & 0.02635 & 0.03013 & -12.54 \\
\hline 383.114 & 4.15 & 190.5 & 0.02619 & 0.03013 & -13.08 \\
\hline 383.501 & 4.15 & 189.9 & 0.02629 & 0.03014 & -12.76 \\
\hline 382.045 & 3.37 & 141.1 & 0.02429 & 0.02809 & -13.54 \\
\hline
\end{tabular}


Table 11. Thermal conductivity for R507A (continued)

\begin{tabular}{|c|c|c|c|c|c|}
\hline $\begin{array}{l}\text { Temperature } \\
\text { (K) }\end{array}$ & $\begin{array}{l}\text { Pressure } \\
(\mathrm{MPa})\end{array}$ & $\begin{array}{c}\text { Density (calc) } \\
\left(\mathrm{kg} / \mathrm{m}^{3}\right)\end{array}$ & $\begin{array}{c}\lambda_{\exp } \\
(\mathrm{W} / \mathrm{m} \cdot \mathrm{K})\end{array}$ & $\begin{array}{c}\lambda_{\text {calc }} \\
(\mathrm{W} / \mathrm{m} \cdot \mathrm{K})\end{array}$ & $\begin{array}{l}\Delta \lambda \\
(\%)\end{array}$ \\
\hline 382.389 & 3.37 & 140.8 & 0.02440 & 0.02811 & -13.21 \\
\hline 382.743 & 3.37 & 140.6 & 0.02440 & 0.02814 & -13.29 \\
\hline 383.133 & 3.37 & 140.2 & 0.02449 & 0.02816 & -13.04 \\
\hline 383.545 & 3.37 & 139.9 & 0.02449 & 0.02819 & -13.13 \\
\hline 382.308 & 2.45 & 93.1 & 0.02314 & 0.02636 & -12.21 \\
\hline 382.691 & 2.45 & 92.8 & 0.02306 & 0.02639 & -12.62 \\
\hline 383.093 & 2.45 & 92.5 & 0.02304 & 0.02643 & -12.82 \\
\hline 383.540 & 2.45 & 92.3 & 0.02308 & 0.02647 & -12.81 \\
\hline 384.004 & 2.45 & 92.1 & 0.02317 & 0.02652 & -12.62 \\
\hline 382.284 & 1.20 & 40.8 & 0.02196 & 0.02534 & -13.32 \\
\hline 382.583 & 1.20 & 40.9 & 0.02211 & 0.02537 & -12.85 \\
\hline 382.919 & 1.21 & 40.9 & 0.02210 & 0.02541 & -13.02 \\
\hline 383.278 & 1.21 & 41.0 & 0.02207 & 0.02545 & -13.27 \\
\hline 383.622 & 1.21 & 41.0 & 0.02219 & 0.02549 & -12.93 \\
\hline 382.322 & 1.15 & 38.8 & 0.02214 & 0.02532 & -12.54 \\
\hline 382.636 & 1.18 & 40.0 & 0.02212 & 0.02536 & -12.78 \\
\hline 382.963 & 1.19 & 40.4 & 0.02211 & 0.02540 & -12.97 \\
\hline 383.297 & 1.17 & 39.5 & 0.02207 & 0.02543 & -13.21 \\
\hline 383.670 & 1.15 & 38.8 & 0.02201 & 0.02546 & -13.55 \\
\hline 382.383 & 1.05 & 35.2 & 0.02198 & 0.02529 & -13.09 \\
\hline 382.702 & 1.07 & 35.9 & 0.02211 & 0.02533 & -12.71 \\
\hline 383.003 & 1.09 & 36.5 & 0.02211 & 0.02537 & -12.84 \\
\hline 383.320 & 1.17 & 39.3 & 0.02207 & 0.02543 & -13.21 \\
\hline 383.661 & 1.16 & 39.0 & 0.02224 & 0.02546 & -12.65 \\
\hline 382.453 & 0.90 & 29.8 & 0.02195 & 0.02525 & -13.09 \\
\hline 382.758 & 0.90 & 29.8 & 0.02201 & 0.02529 & -12.96 \\
\hline 383.102 & 0.90 & 29.8 & 0.02206 & 0.02532 & -12.89 \\
\hline 383.462 & 0.90 & 29.7 & 0.02207 & 0.02536 & -12.98 \\
\hline 383.837 & 0.90 & 29.7 & 0.02205 & 0.02540 & -13.20 \\
\hline 382.560 & 0.62 & 20.2 & 0.02186 & 0.02524 & -13.41 \\
\hline 382.891 & 0.62 & 20.2 & 0.02192 & 0.02528 & -13.29 \\
\hline 383.248 & 0.62 & 20.2 & 0.02206 & 0.02532 & -12.87 \\
\hline 383.622 & 0.62 & 20.2 & 0.02201 & 0.02536 & -13.20 \\
\hline 384.007 & 0.62 & 20.2 & 0.02209 & 0.02540 & -13.03 \\
\hline 382.498 & 0.36 & 11.3 & 0.02200 & 0.02523 & -12.80 \\
\hline 382.837 & 0.36 & 11.3 & 0.02204 & 0.02526 & -12.76 \\
\hline 383.201 & 0.36 & 11.3 & 0.02201 & 0.02530 & -13.02 \\
\hline 383.585 & 0.36 & 11.3 & 0.02206 & 0.02534 & -12.96 \\
\hline 383.992 & 0.36 & 11.3 & 0.02205 & 0.02539 & -13.15 \\
\hline 382.636 & 0.21 & 6.5 & 0.02199 & 0.02524 & -12.88 \\
\hline 382.994 & 0.21 & 6.5 & 0.02193 & 0.02528 & -13.25 \\
\hline 383.377 & 0.21 & 6.5 & 0.02206 & 0.02532 & -12.87 \\
\hline 383.793 & 0.21 & 6.5 & 0.02209 & 0.02536 & -12.91 \\
\hline 384.226 & 0.21 & 6.5 & 0.02220 & 0.02541 & -12.63 \\
\hline 382.806 & 0.10 & 3.2 & 0.02195 & 0.02526 & -13.09 \\
\hline 383.183 & 0.10 & 3.2 & 0.02210 & 0.02530 & -12.64 \\
\hline 383.603 & 0.10 & 3.2 & 0.02206 & 0.02534 & -12.95 \\
\hline 384.037 & 0.10 & 3.2 & 0.02217 & 0.02539 & -12.68 \\
\hline
\end{tabular}


Table 11. Thermal conductivity for R507A (continued)

\begin{tabular}{|c|c|c|c|c|c|}
\hline $\begin{array}{c}\text { Temperature } \\
\text { (K) }\end{array}$ & $\begin{array}{l}\text { Pressure } \\
(\mathrm{MPa})\end{array}$ & $\begin{array}{c}\text { Density (calc) } \\
\left(\mathrm{kg} / \mathrm{m}^{3}\right)\end{array}$ & $\begin{array}{c}\lambda_{\exp } \\
(\mathrm{W} / \mathrm{m} \cdot \mathrm{K})\end{array}$ & $\begin{array}{c}\lambda_{\text {calc }} \\
(\mathrm{W} / \mathrm{m} \cdot \mathrm{K})\end{array}$ & $\begin{array}{l}\Delta \lambda \\
(\%\end{array}$ \\
\hline 384.493 & 0.10 & 3.2 & 0.02218 & 0.02544 & -12.81 \\
\hline 331.883 & 3.93 & 873.9 & 0.05056 & 0.05400 & -6.37 \\
\hline 332.077 & 3.93 & 872.3 & 0.05033 & 0.05393 & -6.68 \\
\hline 332.268 & 3.93 & 870.6 & 0.05043 & 0.05386 & -6.37 \\
\hline 332.460 & 3.93 & 868.8 & 0.05032 & 0.05378 & -6.43 \\
\hline 332.680 & 3.92 & 866.5 & 0.05011 & 0.05368 & -6.66 \\
\hline 331.893 & 3.43 & 853.1 & 0.04949 & 0.05313 & -6.85 \\
\hline 332.074 & 3.43 & 851.2 & 0.04951 & 0.05306 & -6.68 \\
\hline 332.271 & 3.42 & 848.8 & 0.04939 & 0.05296 & -6.75 \\
\hline 332.474 & 3.42 & 846.4 & 0.04931 & 0.05288 & -6.74 \\
\hline 332.686 & 3.42 & 844.1 & 0.04948 & 0.05279 & -6.28 \\
\hline 331.472 & 3.14 & 842.9 & 0.04908 & 0.05277 & -6.99 \\
\hline 331.632 & 3.14 & 841.0 & 0.04915 & 0.05270 & -6.74 \\
\hline 331.818 & 3.13 & 838.5 & 0.04908 & 0.05262 & -6.72 \\
\hline 331.983 & 3.13 & 836.2 & 0.04889 & 0.05254 & -6.96 \\
\hline 332.176 & 3.13 & 833.9 & 0.04887 & 0.05247 & -6.86 \\
\hline 331.470 & 2.92 & 829.9 & 0.04862 & 0.05238 & -7.18 \\
\hline 331.629 & 2.92 & 827.7 & 0.04881 & 0.05232 & -6.71 \\
\hline 331.804 & 2.92 & 825.2 & 0.04873 & 0.05225 & -6.73 \\
\hline 331.990 & 2.92 & 822.1 & 0.04828 & 0.05217 & -7.45 \\
\hline 332.178 & 2.92 & 819.2 & 0.04845 & 0.05209 & -7.00 \\
\hline 401.986 & 19.19 & 834.2 & 0.05515 & 0.05670 & -2.73 \\
\hline 402.170 & 19.20 & 833.7 & 0.05513 & 0.05668 & -2.74 \\
\hline 402.372 & 19.20 & 833.0 & 0.05475 & 0.05667 & -3.38 \\
\hline 402.589 & 19.20 & 832.3 & 0.05511 & 0.05664 & -2.71 \\
\hline 402.814 & 19.19 & 831.4 & 0.05480 & 0.05662 & -3.21 \\
\hline 402.062 & 16.55 & 789.8 & 0.05226 & 0.05439 & -3.92 \\
\hline 402.254 & 16.55 & 789.1 & 0.05238 & 0.05438 & -3.68 \\
\hline 402.472 & 16.56 & 788.3 & 0.05209 & 0.05436 & -4.17 \\
\hline 402.689 & 16.56 & 787.5 & 0.05231 & 0.05434 & -3.73 \\
\hline 402.925 & 16.55 & 786.4 & 0.05234 & 0.05430 & -3.62 \\
\hline 401.955 & 14.20 & 739.1 & 0.04969 & 0.05204 & -4.51 \\
\hline 402.145 & 14.21 & 738.3 & 0.04971 & 0.05202 & -4.44 \\
\hline 402.364 & 14.21 & 737.4 & 0.04934 & 0.05200 & -5.11 \\
\hline 402.571 & 14.21 & 736.6 & 0.04945 & 0.05198 & -4.86 \\
\hline 402.808 & 14.21 & 735.4 & 0.04955 & 0.05195 & -4.61 \\
\hline 402.028 & 12.39 & 686.2 & 0.04710 & 0.04987 & -5.56 \\
\hline 402.230 & 12.39 & 685.2 & 0.04710 & 0.04985 & -5.52 \\
\hline 402.456 & 12.40 & 684.2 & 0.04678 & 0.04983 & -6.12 \\
\hline 402.691 & 12.40 & 683.1 & 0.04720 & 0.04980 & -5.23 \\
\hline 402.925 & 12.40 & 682.0 & 0.04713 & 0.04978 & -5.32 \\
\hline 401.908 & 11.06 & 635.9 & 0.04490 & 0.04802 & -6.50 \\
\hline 402.133 & 11.06 & 634.8 & 0.04503 & 0.04800 & -6.18 \\
\hline 402.336 & 11.06 & 633.7 & 0.04506 & 0.04797 & -6.07 \\
\hline 402.559 & 11.06 & 632.5 & 0.04472 & 0.04795 & -6.73 \\
\hline 402.809 & 11.06 & 630.9 & 0.04494 & 0.04791 & -6.20 \\
\hline
\end{tabular}


Table 11. Thermal conductivity for R507A (continued)

\begin{tabular}{|c|c|c|c|c|c|}
\hline $\begin{array}{l}\text { Temperature } \\
\text { (K) }\end{array}$ & $\begin{array}{l}\text { Pressure } \\
(\mathrm{MPa})\end{array}$ & $\begin{array}{c}\text { Density (calc) } \\
\left(\mathrm{kg} / \mathrm{m}^{3}\right)\end{array}$ & $\begin{array}{c}\lambda_{\exp } \\
(\mathrm{W} / \mathrm{m} \cdot \mathrm{K})\end{array}$ & $\begin{array}{c}\lambda_{\text {calc }} \\
(\mathrm{W} / \mathrm{m} \cdot \mathrm{K})\end{array}$ & $\begin{array}{l}\Delta \lambda \\
(\%)\end{array}$ \\
\hline 401.974 & 10.01 & 584.6 & 0.04289 & 0.04631 & -7.39 \\
\hline 402.206 & 10.01 & 583.3 & 0.04317 & 0.04628 & -6.73 \\
\hline 402.420 & 10.01 & 582.1 & 0.04286 & 0.04626 & -7.34 \\
\hline 402.657 & 10.02 & 580.7 & 0.04289 & 0.04622 & -7.21 \\
\hline 402.913 & 10.02 & 579.1 & 0.04281 & 0.04619 & -7.31 \\
\hline 401.977 & 9.16 & 533.3 & 0.04090 & 0.04468 & -8.46 \\
\hline 402.129 & 9.16 & 532.2 & 0.04112 & 0.04465 & -7.91 \\
\hline 402.324 & 9.16 & 531.1 & 0.04138 & 0.04463 & -7.27 \\
\hline 402.495 & 9.16 & 530.1 & 0.04088 & 0.04460 & -8.35 \\
\hline 402.667 & 9.16 & 529.2 & 0.04083 & 0.04458 & -8.41 \\
\hline 402.054 & 8.45 & 481.9 & 0.03920 & 0.04302 & -8.89 \\
\hline 402.215 & 8.44 & 480.8 & 0.03932 & 0.04299 & -8.55 \\
\hline 402.422 & 8.44 & 479.5 & 0.03902 & 0.04296 & -9.17 \\
\hline 402.606 & 8.45 & 478.5 & 0.03890 & 0.04293 & -9.40 \\
\hline 402.800 & 8.45 & 477.4 & 0.03910 & 0.04291 & -8.87 \\
\hline 402.000 & 7.84 & 434.2 & 0.03753 & 0.04139 & -9.32 \\
\hline 402.182 & 7.84 & 433.2 & 0.03744 & 0.04136 & -9.47 \\
\hline 402.363 & 7.84 & 432.3 & 0.03743 & 0.04133 & -9.44 \\
\hline 402.545 & 7.84 & 431.4 & 0.03723 & 0.04130 & -9.86 \\
\hline 402.734 & 7.84 & 430.3 & 0.03708 & 0.04127 & -10.16 \\
\hline 401.936 & 7.26 & 386.3 & 0.03543 & 0.03960 & -10.54 \\
\hline 402.145 & 7.26 & 385.2 & 0.03546 & 0.03957 & -10.39 \\
\hline 402.310 & 7.26 & 384.5 & 0.03545 & 0.03955 & -10.37 \\
\hline 402.494 & 7.26 & 383.7 & 0.03546 & 0.03953 & -10.29 \\
\hline 402.701 & 7.26 & 382.7 & 0.03526 & 0.03950 & -10.73 \\
\hline 402.116 & 6.69 & 338.1 & 0.03362 & 0.03770 & -10.83 \\
\hline 402.300 & 6.69 & 337.3 & 0.03363 & 0.03768 & -10.75 \\
\hline 402.501 & 6.69 & 336.4 & 0.03358 & 0.03765 & -10.81 \\
\hline 402.711 & 6.69 & 335.5 & 0.03359 & 0.03763 & -10.72 \\
\hline 402.930 & 6.69 & 334.7 & 0.03351 & 0.03760 & -10.89 \\
\hline 402.243 & 6.11 & 291.9 & 0.03176 & 0.03580 & -11.29 \\
\hline 402.457 & 6.11 & 291.2 & 0.03189 & 0.03579 & -10.89 \\
\hline 402.677 & 6.11 & 290.4 & 0.03171 & 0.03577 & -11.35 \\
\hline 402.902 & 6.11 & 289.6 & 0.03166 & 0.03575 & -11.44 \\
\hline 403.140 & 6.11 & 288.9 & 0.03174 & 0.03574 & -11.19 \\
\hline 402.162 & 5.48 & 245.9 & 0.02960 & 0.03390 & -12.69 \\
\hline 402.369 & 5.48 & 245.5 & 0.02961 & 0.03390 & -12.66 \\
\hline 402.593 & 5.48 & 245.0 & 0.02964 & 0.03390 & -12.57 \\
\hline 402.828 & 5.48 & 244.3 & 0.02965 & 0.03389 & -12.51 \\
\hline 403.082 & 5.47 & 243.7 & 0.02957 & 0.03388 & -12.73 \\
\hline 402.322 & 4.68 & 194.4 & 0.02809 & 0.03191 & -11.98 \\
\hline 402.578 & 4.68 & 194.2 & 0.02803 & 0.03193 & -12.20 \\
\hline 402.808 & 4.68 & 193.8 & 0.02811 & 0.03193 & -11.98 \\
\hline 403.077 & 4.68 & 193.3 & 0.02799 & 0.03194 & -12.37 \\
\hline 403.335 & 4.68 & 192.8 & 0.02801 & 0.03195 & -12.32 \\
\hline 402.570 & 3.78 & 144.7 & 0.02651 & 0.03023 & -12.30 \\
\hline 402.818 & 3.78 & 144.6 & 0.02656 & 0.03025 & -12.20 \\
\hline 403.085 & 3.78 & 144.5 & 0.02652 & 0.03027 & -12.40 \\
\hline
\end{tabular}


Table 11. Thermal conductivity for R507A (continued)

$\begin{array}{cccccr}\text { Temperature } & \text { Pressure } & \text { Density (calc) } & \lambda_{\text {exp }} & \lambda_{\text {calc }} & \Delta \lambda \\ (\mathrm{K}) & (\mathrm{MPa}) & \left(\mathrm{kg} / \mathrm{m}^{3}\right) & (\mathrm{W} / \mathrm{m} \cdot \mathrm{K}) & (\mathrm{W} / \mathrm{m} \cdot \mathrm{K}) & (\%) \\ & & & & & \\ 403.385 & 3.78 & 144.3 & 0.02656 & 0.03030 & -12.34 \\ 403.694 & 3.78 & 143.9 & 0.02655 & 0.03032 & -12.43 \\ 402.555 & 2.71 & 95.5 & 0.02511 & 0.02865 & -12.34 \\ 402.847 & 2.71 & 95.3 & 0.02509 & 0.02867 & -12.50 \\ 403.128 & 2.71 & 95.3 & 0.02525 & 0.02871 & -12.04 \\ 403.438 & 2.72 & 95.3 & 0.02515 & 0.02874 & -12.49 \\ 403.764 & 2.72 & 95.2 & 0.02524 & 0.02878 & -12.29 \\ 402.894 & 1.44 & 46.5 & 0.02428 & 0.02772 & -12.42 \\ 403.205 & 1.45 & 46.6 & 0.02439 & 0.02776 & -12.14 \\ 403.536 & 1.45 & 46.6 & 0.02435 & 0.02780 & -12.41 \\ 403.902 & 1.45 & 46.6 & 0.02437 & 0.02784 & -12.47 \\ 404.269 & 1.45 & 46.6 & 0.02434 & 0.02788 & -12.70 \\ 402.663 & 1.13 & 35.5 & 0.02427 & 0.02754 & -11.86 \\ 402.995 & 1.13 & 35.8 & 0.02415 & 0.02758 & -12.43 \\ 403.333 & 1.15 & 36.1 & 0.02421 & 0.02762 & -12.35 \\ 403.666 & 1.16 & 36.4 & 0.02434 & 0.02766 & -12.01 \\ 404.063 & 1.14 & 35.8 & 0.02422 & 0.02770 & -12.56 \\ 402.985 & 0.47 & 14.2 & 0.02421 & 0.02745 & -11.79 \\ 403.323 & 0.47 & 14.1 & 0.02445 & 0.02748 & -11.03 \\ 402.572 & 0.47 & 14.3 & 0.02423 & 0.02740 & -11.57 \\ 402.891 & 0.47 & 14.2 & 0.02427 & 0.02744 & -11.54 \\ 403.219 & 0.47 & 14.2 & 0.02424 & 0.02747 & -11.76 \\ 403.595 & 0.47 & 14.2 & 0.02418 & 0.02751 & -12.11 \\ 403.975 & 0.47 & 14.2 & 0.02438 & 0.02755 & -11.52 \\ 402.659 & 0.31 & 9.4 & 0.02422 & 0.02741 & -11.63 \\ 402.995 & 0.31 & 9.4 & 0.02430 & 0.02744 & -11.46 \\ 403.347 & 0.31 & 9.4 & 0.02438 & 0.02748 & -11.29 \\ 403.723 & 0.31 & 9.4 & 0.02431 & 0.02752 & -11.68 \\ 404.125 & 0.31 & 9.4 & 0.02443 & 0.02757 & -11.38 \\ 402.759 & 0.21 & 6.3 & 0.02438 & 0.02742 & -11.08 \\ 403.084 & 0.21 & 6.3 & 0.02457 & 0.02745 & -10.50 \\ 403.452 & 0.21 & 6.3 & 0.02442 & 0.02749 & -11.18 \\ 403.870 & 0.21 & 6.3 & 0.02435 & 0.02754 & -11.58 \\ 404.292 & 0.21 & 6.3 & 0.02448 & 0.02758 & -11.25 \\ 402.900 & 0.11 & 3.1 & 0.02433 & 0.02743 & -11.31 \\ 403.271 & 0.11 & 3.1 & 0.02432 & 0.02747 & -11.47 \\ 403.673 & 0.11 & 3.1 & 0.02439 & 0.02752 & -11.36 \\ 404.111 & 0.11 & 3.1 & 0.02448 & 0.02756 & -11.19 \\ 404.533 & 0.11 & 3.1 & 0.02444 & 0.02761 & -11.48\end{array}$


Table 11. Thermal conductivity for R507A (continued)

$\begin{array}{crrccr}\text { Temperature } & \begin{array}{c}\text { Pressure } \\ (\mathrm{K})\end{array} & \begin{array}{c}\text { Density }(\text { calc }) \\ (\mathrm{MPa})\end{array} & \begin{array}{c}\lambda \text { exp } \\ \left(\mathrm{kg} / \mathrm{m}^{3}\right)\end{array} & \begin{array}{c}\lambda / \mathrm{W} \cdot \mathrm{K}) \\ (\mathrm{W} / \mathrm{m} \cdot \mathrm{K})\end{array} & \begin{array}{c}\Delta \\ (\%)\end{array} \\ 302.386 & 0.30 & 12.2 & 0.01439 & 0.01711 & -15.89 \\ 302.802 & 0.29 & 11.8 & 0.01440 & 0.01715 & -16.02 \\ 303.257 & 0.28 & 11.5 & 0.01439 & 0.01719 & -16.29 \\ 303.724 & 0.27 & 11.1 & 0.01442 & 0.01723 & -16.33 \\ 304.236 & 0.26 & 10.8 & 0.01444 & 0.01728 & -16.45 \\ 302.312 & 0.10 & 4.2 & 0.01480 & 0.01709 & -13.40 \\ 302.746 & 0.10 & 4.1 & 0.01482 & 0.01713 & -13.49 \\ 303.203 & 0.10 & 4.1 & 0.01478 & 0.01717 & -13.94 \\ 303.681 & 0.10 & 4.1 & 0.01477 & 0.01722 & -14.23 \\ 304.204 & 0.10 & 4.1 & 0.01476 & 0.01727 & -14.54 \\ 317.168 & 0.30 & 11.7 & 0.01552 & 0.01854 & -16.27 \\ 317.537 & 0.30 & 11.7 & 0.01560 & 0.01857 & -16.00 \\ 317.930 & 0.30 & 11.6 & 0.01560 & 0.01861 & -16.18 \\ 318.342 & 0.30 & 11.6 & 0.01560 & 0.01865 & -16.36 \\ 318.790 & 0.30 & 11.5 & 0.01564 & 0.01869 & -16.34 \\ 317.285 & 0.21 & 7.9 & 0.01560 & 0.01854 & -15.87 \\ 317.660 & 0.21 & 7.9 & 0.01563 & 0.01858 & -15.87 \\ 318.064 & 0.20 & 7.8 & 0.01565 & 0.01862 & -15.94 \\ 318.490 & 0.20 & 7.8 & 0.01565 & 0.01866 & -16.13 \\ 318.955 & 0.20 & 7.8 & 0.01568 & 0.01871 & -16.17 \\ 317.079 & 0.10 & 4.0 & 0.01582 & 0.01852 & -14.57 \\ 317.455 & 0.10 & 4.0 & 0.01582 & 0.01856 & -14.74 \\ 317.857 & 0.11 & 4.0 & 0.01582 & 0.01859 & -14.92 \\ 318.294 & 0.10 & 4.0 & 0.01582 & 0.01864 & -15.12 \\ 318.769 & 0.10 & 3.9 & 0.01582 & 0.01868 & -15.33 \\ 331.927 & 0.30 & 11.3 & 0.01685 & 0.01999 & -15.73 \\ 332.263 & 0.30 & 11.2 & 0.01692 & 0.02003 & -15.52 \\ 332.609 & 0.30 & 11.2 & 0.01691 & 0.02006 & -15.71 \\ 332.994 & 0.30 & 11.2 & 0.01691 & 0.02010 & -15.88 \\ 333.381 & 0.30 & 11.2 & 0.01698 & 0.02014 & -15.69 \\ 332.021 & 0.20 & 7.4 & 0.01694 & 0.02000 & -15.30 \\ 332.362 & 0.20 & 7.4 & 0.01692 & 0.02003 & -15.54 \\ 332.745 & 0.20 & 7.4 & 0.01693 & 0.02007 & -15.65 \\ 333.131 & 0.20 & 7.3 & 0.01693 & 0.02011 & -15.82 \\ 333.540 & 0.20 & 7.3 & 0.01696 & 0.02015 & -15.84 \\ 332.157 & 0.10 & 3.7 & 0.01703 & 0.02001 & -14.90 \\ 332.526 & 0.10 & 3.7 & 0.01701 & 0.02005 & -15.15 \\ 332.940 & 0.10 & 3.7 & 0.01703 & 0.02009 & -15.23 \\ 333.342 & 0.10 & 3.7 & 0.01706 & 0.02013 & -15.25 \\ 333.789 & 0.10 & 3.7 & 0.01705 & 0.02017 & -15.49\end{array}$




\section{MIXTURE VISCOSITY MEASUREMENTS}

Experimental. Viscosity measurements of the blends R507A and R410A were carried out in the torsional crystal viscometer at two subcritical and three supercritical isotherms with pressures up to $82 \mathrm{MPa}$. The subcritical measurements were conducted at the nominal temperatures $300 \mathrm{~K}$ (liquid only) and $340 \mathrm{~K}$ (liquid and vapor). Supercritical measurements included the nominal temperatures $360 \mathrm{~K}, 390 \mathrm{~K}$, and $420 \mathrm{~K}$.

As described in Sections 4 and 6, the mixtures were prepared gravimetrically using high-purity components. The actual composition of the R410A (R32/125) cylinder used in the viscosity measurements was 0.50001/0.49999 mass fraction, and that of the R507A (R125/143a) was $0.49983 / 0.50017$ mass fraction. The uncertainties in compositions are \pm 0.00002 mass fraction.

The majority of the measurements were carried out in a torsional crystal viscometer. The measuring element in this instrument is a cylindrical quartz crystal immersed in the fluid of interest. The crystal is caused to oscillate in a torsional motion at its resonant frequency by an applied electric field. The resonant frequency and the bandwidth of the resonance depend on the viscosity-density product of the surrounding fluid. The technique and the details of the instrument are given by Diller and Frederick (1989).

The working equation for absolute viscosity measurements with the torsional crystal viscometer in the frequency domain is given by

$$
\eta \times \rho=\left(\frac{m}{S}\right)^{2}\left(\pi f^{*}\right)\left(\frac{\Delta f}{f^{*}}-\frac{\Delta f_{0}}{f_{0}^{*}}\right)^{2} .
$$

Here, $\eta$ denotes the viscosity and $\rho$ the density of the fluid; $m$ and $S$ are the mass and surface area of the crystal; $f^{*}$ is the resonant frequency and $\Delta f$ is the bandwidth of the resonance. Subscript 0 indicates vacuum conditions. Since this technique measures the viscosity-density product $(\eta \times \rho)$, the density of the fluid must be obtained separately in order to determine the viscosity. Thus, the uncertainty of the density propagates directly into the uncertainty of the viscosity measurement. In this work, densities of the refrigerant blends were calculated for the measured pressures and temperatures with REFPROP (Lemmon et al. 2002). The estimated uncertainty in density is $\pm 0.1 \%$.

The viscosity measurements were carried out with the cylindrical quartz crystal denoted as “crystal D" (diameter $d=3.046 \mathrm{~mm}$, length $L=50.076 \mathrm{~mm}$, mass $m=0.9662 \mathrm{~g}$ ) in the transducer of the viscometer. The resonance of the crystal in vacuo depends on the applied voltage to drive the torsional crystal vibration and on temperature. The change of the resonant frequency $f_{0} *$ and of the resonance bandwidth $\Delta f_{0}$ with these variables was measured after the measurements of the refrigerant blends with drive voltages from $5 \mathrm{mV}$ to $1.1 \mathrm{~V}$ in the temperature range from $300 \mathrm{~K}$ to $460 \mathrm{~K}$. The results confirmed previous similar measurements in 1996 and in 1998 which indicates a remarkable long-term stability of the transducer. The data from the vacuum measurements of the transducer were correlated for each drive voltage by polynomials in temperature. The resonant frequency $f_{0} *$ follows closely a quadratic polynomial with a maximum at approximately $369 \mathrm{~K}$. The logarithm of the bandwidth $\Delta f_{0}$ can be regressed in terms of a cubic polynomial in temperature. These correlations were used in the analysis of the measurements of the refrigerant blends. The accurate determination of the voltage and temperature dependence of the crystal vibration in vacuo made it possible to expand the range of operation of the viscometer from saturated or compressed liquids to dilute gases, thus covering a wide range of fluid states with one transducer. 
The measurement sequence was designed to use the limited amount of sample as economically as possible. It began by condensing sample from the aluminum supply cylinders through the heated filling system of the viscometer into the stainless-steel high pressure measuring cell which was cooled with liquid nitrogen to less than $200 \mathrm{~K}$. The filling of the cell could be monitored by setting the impedance analyzer to $39 \mathrm{kHz}$ and reading the complex admittance $Y=G+j B$ of the torsional crystal transducer in the cell. The susceptance $B$ indicates the permittivity of the dielectric between the electrodes of the transducer. The reference is its wellestablished value $B_{0}=34.17 \mu \mathrm{S}$ in vacuo. When the transducer was fully immersed in liquid, the cell was returned to $300 \mathrm{~K}$ and the maximum pressure by isochoric heating. The semi-automated viscometer was programmed with the desired target pressures, and it carried out the measurements at constant temperature under computer control to a pressure near saturation. The cell was then heated to $420 \mathrm{~K}$ to raise the pressure to the starting pressure for that isotherm. When completed, the viscometer was refilled for each of the remaining isotherms at $340 \mathrm{~K}$, $360 \mathrm{~K}$, and $390 \mathrm{~K}$.

Of the refrigerant blends in this work, R507A was measured first and R410A second. The measurement protocol for fluids comprises frequency scans of the transducer resonance in the sample fluid with varying drive voltages at every pressure of each isotherm. Resolving the dependence of the resonance on the drive voltage allows extrapolation to the resonance at zero drive voltage which is consistent with the definition of Newtonian viscosity as a property characterizing momentum transport in the limit of zero shear. Due to the resolution limits of the impedance analyzer used with the instrument, the majority of the measurements was limited to drive voltages from $0.1 \mathrm{~V}$ to $1.1 \mathrm{~V}$. Lower voltages to $10 \mathrm{mV}$ could be applied only in a few cases of dilute gases. For reasons of consistency, all the results reported in this work were derived from resonance scans with drive voltages of $0.1 \mathrm{~V}$. The uncertainty of measurements with the torsional crystal viscometer typically does not exceed $\pm 2 \%$.

The experimental viscosities measured with the torsional crystal viscometer were compared with viscosities calculated with the extended corresponding states model implemented in REFPROP (i.e., the model of Klein et al. 1997). They were also compared with literature data. In the following, the results will be presented for each blend and their reliability will be discussed.

Results for R507A. The impedence analyzer used with the instrument can also provide a measurement of the conductance G (measured in units of Siemens) at the resonant frequency of approximately $39 \mathrm{kHz}$. Examination of the conductance values is important for polar fluids, such as the HFC refrigerants, because measurements of fluids with a high background conductance $(G>1 \mu \mathrm{S})$ may be influenced by electroviscous effects because the sample fluid is exposed to the applied drive voltage.

Figure 10 shows the measured conductances $G$ at $39 \mathrm{kHz}$ of the blend R507A as a function of density. Any increase of the conductance beyond that of the instrument circuitry under vacuum indicates the presence of charge carriers in the sample. No increase of the conductances of R507A occurs up to a density of about $500 \mathrm{~kg} / \mathrm{m}^{3}$. At higher densities the conductances increase with both density and temperature. While the increase is monotone at $360 \mathrm{~K}$ and above, a flattening is observed on the isotherms at $300 \mathrm{~K}$ and $340 \mathrm{~K}$ beginning at about $1100 \mathrm{~kg} / \mathrm{m}^{3}$. The highest conductance of $G=0.62 \mu \mathrm{S}$ was measured at the highest pressure of the $390 \mathrm{~K}$-isotherm. The scatter of the conductances at $360 \mathrm{~K}$ was caused by intermittent problems with an electrical connection in the instrument circuitry.

The viscosities for the blend R507A that were measured in the torsional crystal viscometer are tabulated in Table 12. Their density dependence is displayed in Figure 11. Due to the measurements at supercritical temperatures they cover the entire density range from $0.09 \mathrm{~kg} / \mathrm{m}^{3}$ to $1300 \mathrm{~kg} / \mathrm{m}^{3}$. Figure 11 shows that the viscosity data aggregate along a single line with a barely discernible temperature dependence. The weak temperature dependence is revealed when the 
measured viscosities are compared with data that are calculated with the extended corresponding states model in REFPROP. Figure 12 illustrates the percent deviations between experimental and calculated viscosities of R507A. A significant systematic deviation with density is observed. Smaller systematic deviations from isotherm to isotherm are also seen. The scatter from point to point along a given isotherm are well within the \pm 2 uncertainty of the measurements. These results reveal a deficiency in the extended corresponding states model.

The present measurements constitute a significant improvement of our knowledge of the viscosity of R507A. This is documented in Figure 13 which compares the previously available literature data of the binary system R143a + R125 with the extended corresponding states model in REFPROP. The saturated liquid measurements of Heide and Schenk (1996) deviate from the calculated viscosities between $-4.5 \%$ and $15 \%$. The gas measurements of Nabizadeh and Mayinger (1999) exhibit the same systematic deviations that exist in all their results. They agree with the calculated data only in the limit of zero density and show increasing deviations up to $14 \%$ with increasing density. The surface light scattering measurements of Fröba et al. (2001) on saturated liquid R507A deviate from the calculated data between $-9.3 \%$ and $3.8 \%$. In summary, all literature data for R507A deviate from the calculated viscosities more than their estimated uncertainties which suggests that these measurements were affected by systematic errors.

Results for R410A. Figure 14 shows the measured conductances $G$ at $39 \mathrm{kHz}$ of the blend R410A as a function of density. As in the case of R507A no increase of the conductances occurs in R410A up to a density of about $500 \mathrm{~kg} / \mathrm{m}^{3}$. At higher densities the conductances of R410A are in strong contrast to the low levels observed in R507A (Figure 10). The density and temperature dependence is much more pronounced and the highest measured conductance of $G=5.72 \mu \mathrm{S}$ at $420 \mathrm{~K}$ and $1050 \mathrm{~kg} / \mathrm{m}^{3}$ is more than nine times higher than the highest conductance measured in R507A. This observation is consistent with our previous studies of pure R32 and of binary systems that contained R32. Elevated electrical conductances were observed in all cases, the reasons of which have not yet been identified in detail.

Based on molecular dynamics simulations, Lísal and Vacek (1996) suggested that the polar R32 molecules associate closely in the liquid state via $\mathrm{H}-\mathrm{F}$ bonds. It is not clear if these associations are strong enough to enable electrical conduction via electron transport. Rather, the electrical conductances observed in our studies indicate the presence of a significant amount of charge carriers in fluids containing R32. Sample contaminations with ionic impurities can be largely excluded as the reason for these conductances because our studies used samples that were independently prepared from various pure fluids, at different times, and in different containers. Thus the charge carriers must originate from the R32 itself by dissociation. Unfortunately, no other quantitative investigations have been carried out that would help to elucidate molecular interactions in fluids containing R32. Barão et al. (1997) and Abbott et al. (1999) measured permittivities of hydrofluorocarbons including R32. Suspiciously, both reports contain complete results only for the other compounds studied but not for R32.

In conclusion, the elevated electrical conductances of R410A that were measured in this work indicate the presence of charge carriers in the sample. The presence of polar molecules and charge carriers influences the operation of the torsional crystal viscometer on two counts. First, the charge carriers follow the applied ac voltage that is transmitted from the electrodes across the fluid sample to drive the torsional vibration of the piezoelectric crystal. Relaxation times of molecular reorientations on the order of picoseconds are much shorter than the period of the alternating voltage which is in the millisecond range. Secondly, the charge carriers are alternatingly attracted and repelled by the oscillating surface charge of the piezoelectric crystal that is generated by its torsional mechanical deformation. This generates a secondary flow perpendicular to the crystal surface that is superimposed to the primary shear flow of the sample fluid tangential to the crystal surface. The secondary flow adds an electroviscous component to 
the friction in the fluid which increases with the number of charge carriers. Consequently, viscosities measured with the torsional crystal viscometer on electrically conducting fluids are systematically higher than viscosities measured in instruments where the sample is not exposed to an alternating electric field. The systematic deviations are directly proportional to the number of charge carriers which in turn is characterized by the measured electrical conductance.

The viscosities measured in the torsional crystal viscometer are tabulated in Table 13. Their density dependence is displayed in Figure 15; the saturated liquid measurements are from the measurements in the capillary viscometer described below.

For R410A, the temperature dependence of the viscosity is more noticeable than in the results for R507A. A magnified view is offered in the deviation plot, Figure 16. As with R507A, there are strong systematic deviations between the data and the extended corresponding states model. For $\mathrm{R} 410 \mathrm{~A}$, the temperature dependence is somewhat more complicated. In addition to deficiencies in the mixture model, the larger deviations for R410A compared to R507A may be due to the increase of the electrical conductances (Figure 14). The experimental results represent the combined effect of molecular friction and electroviscous friction but the working theory of the instrument accounts only for molecular friction.

To corroborate these results, the viscosity of the refrigerant blend R410A was also measured in a sealed gravitational capillary viscometer between $242 \mathrm{~K}$ and $320 \mathrm{~K}$. This instrument measures the viscosity of the saturated liquid. It is described in detail by Laesecke et al. (1999). The viscometer was recalibrated by measurements on toluene at 320,335 , and $350 \mathrm{~K}$. The calibration constant obtained was $C_{1}=(6.277 \pm 0.067) \cdot 10^{-12} \mathrm{~m}^{3} / \mathrm{s}^{2}$. This value is consistent with our earlier calibrations of this instrument. The calculations were based on the measured bulk density $\rho_{\text {bulk }}=$ $343.4 \mathrm{~kg} / \mathrm{m}^{3}$ of the sample in the viscometer. The contribution of the uncertainty of the bulk density to the experimental uncertainty of the viscosity measurements was assessed by comparing the calculated sample properties at the measured bulk density with those at bulk densities $10 \%$ below and above that value. The resulting variations in the saturated liquid and vapor density ranged from $-0.014 \%$ to $0.017 \%$ and from $-0.055 \%$ to $0.067 \%$, respectively. Thus, the data analysis is rather insensitive to the uncertainty of the bulk density. The expanded uncertainty of the present measurements is estimated at $\pm 2.4 \%$ (coverage factor of 2 ), the same level as in our previous measurements of the similar system R32 + R134a.

The results from the capillary viscometer are presented in Table 14 and depicted in Figure 15. This table gives the experimental temperatures and the estimated densities of saturated liquid and vapor that were calculated using REFPROP. Also included are viscosities calculated with the extended corresponding states model of Klein et al. (1997) (i.e., the model in REFPROP) and the percent deviations between the experimental results and the calculated data. Systematic deviations as a function of density are revealed in Figure 16. But, again, the point-to-point scatter of the data are small.

Deviations of literature data for the viscosity of the binary system R32 + R125 are shown in Figure 17 relative to data calculated with the extended corresponding states model in REFPROP. The first measurements of a mixture of $0.6 \mathrm{R} 32+0.4 \mathrm{R} 125$ (by mass) with a reported uncertainty of $\pm 1.2 \%$ were published by Bivens et al. (1993). Figure 17 shows agreement between these data and the predicted viscosities within the uncertainty estimate except for the data point at $253.15 \mathrm{~K}$ which deviates by about $5 \%$. Heide and Schenk (1996) measured three binary blends at mass fractions of $\mathrm{R} 32$ of $0.753,0.51$, and 0.251 with a falling-body viscometer. The uncertainty was reported to be $\pm 2 \%$ for a viscosity of $0.2 \mathrm{mPa} \cdot \mathrm{s}$. This corresponds to the viscosity of saturated liquid R410A at approximately $259 \mathrm{~K}$. The results of Heide and Schenk do agree with the predicted viscosities within that margin and at such temperatures. However, the deviations increase to almost $-4 \%$ at lower temperatures and to $-18 \%$ at temperatures above $313 \mathrm{~K}$. Two laboratories reported measurements in the gas region with oscillating disk 
viscometers. The results of Nabizadeh and Mayinger (1999) exhibit similar systematic density deviations as their data for R507A (Figure 13) except the limiting value at zero density is offset by about $-3 \%$. Even higher deviations are displayed by their saturated vapor data which are extrapolated from their measurements. The appropriateness of the extrapolation method appears questionable. On the other hand, their results are supported by the measurements of Yokoyama et al. (2001) which exhibit almost congruent deviations from the calculated viscosities. This agreement between independent results of two laboratories suggests that the extended corresponding states model in REFPROP does not capture correctly the molecular interactions in the binary system R32 + R125.

The present work contributes a substantial number of new data that fills the existing data gap for this binary system in the density range from $300 \mathrm{~kg} / \mathrm{m}^{3}$ to $800 \mathrm{~kg} / \mathrm{m}^{3}$. The negative deviations below $300 \mathrm{~kg} / \mathrm{m}^{3}$ are consistent with the results of the oscillating disk measurements of Nabizadeh and Mayinger (1999) and of Yokoyama et al. (2001). They will contribute to the refinement of the mixture model in REFPROP. The results in the liquid and compressed liquid region (at densities above $900 \mathrm{~kg} / \mathrm{m}^{3}$ for the isotherms $300 \mathrm{~K}$ to $390 \mathrm{~K}$ and above $700 \mathrm{~kg} / \mathrm{m}^{3}$ at $420 \mathrm{~K}$ ) form the basis to extend the working theory of the torsional crystal viscometer to electrically conducting fluids. 


\section{G / $\mu S$}

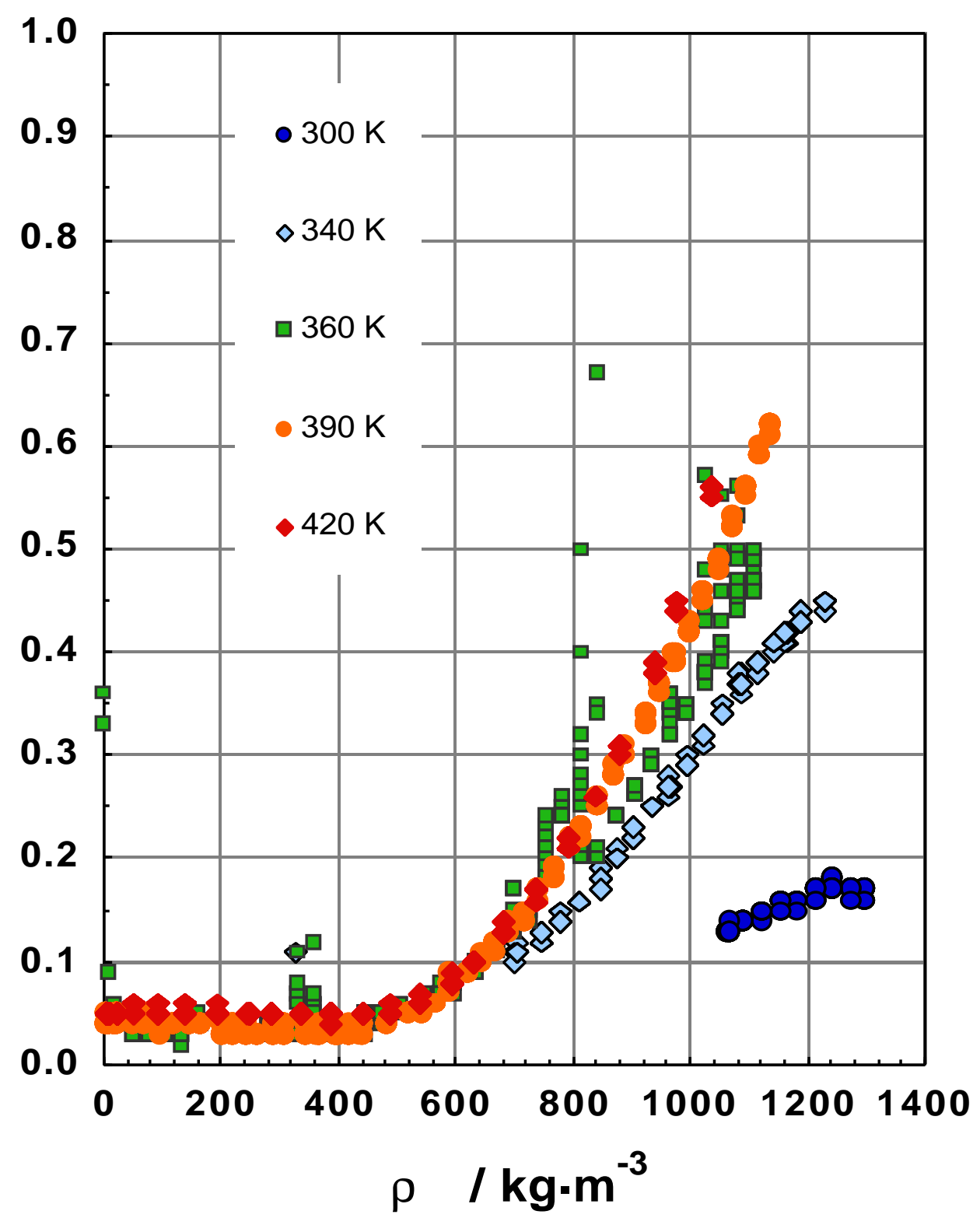

Figure 10. Conductances $G$ at $39 \mathrm{kHz}$ of the mixture R507A (0.5 R143a + $0.5 \mathrm{R} 125$ by mass) as measured in the torsional crystal viscometer. 


\section{$\eta / \mathrm{mPa} \cdot \mathbf{s}$}

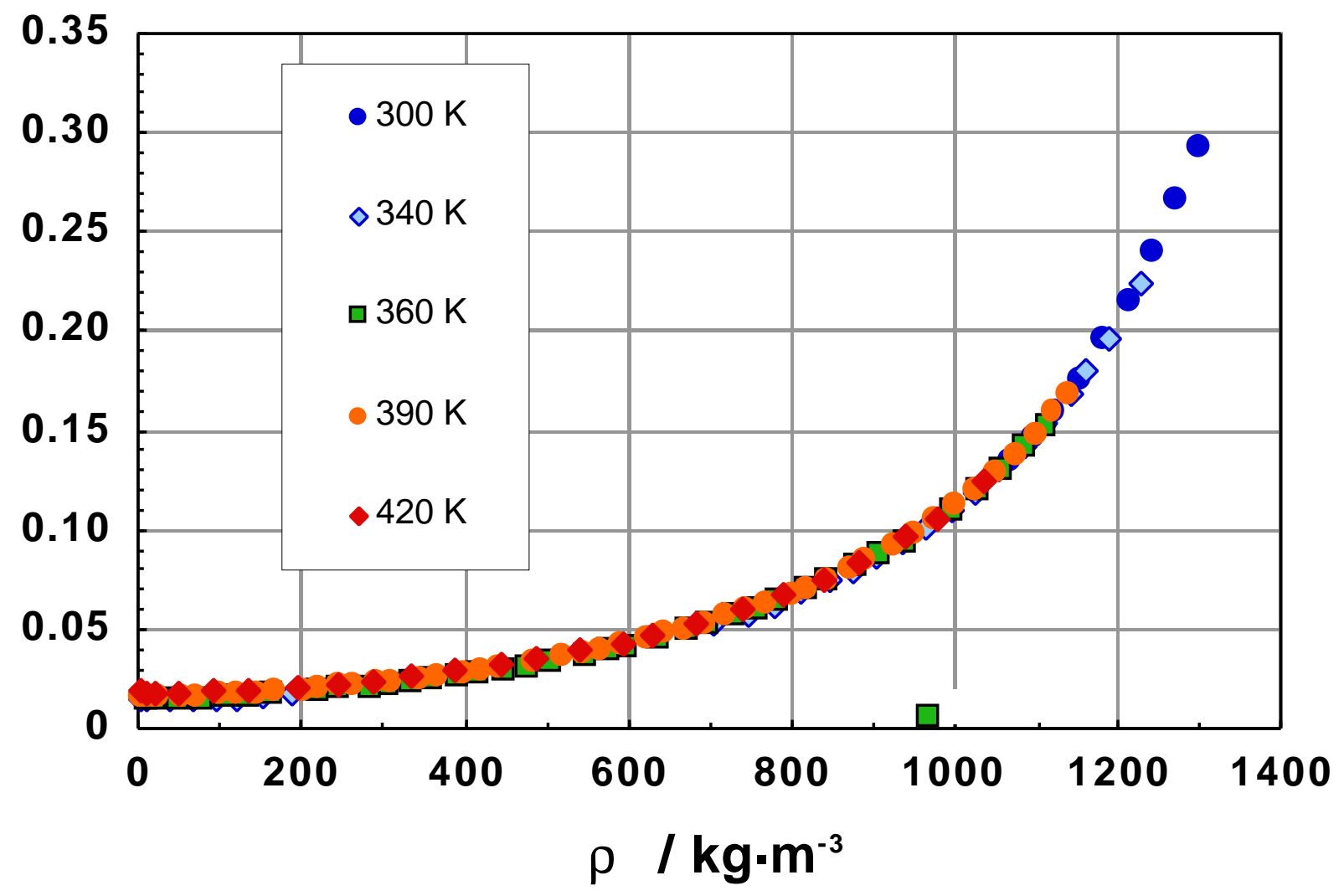

Figure 11. Density dependence of experimental viscosities of the mixture R507A (0.5 R143a + $0.5 \mathrm{R} 125$ by mass) as measured in the torsional crystal viscometer. 


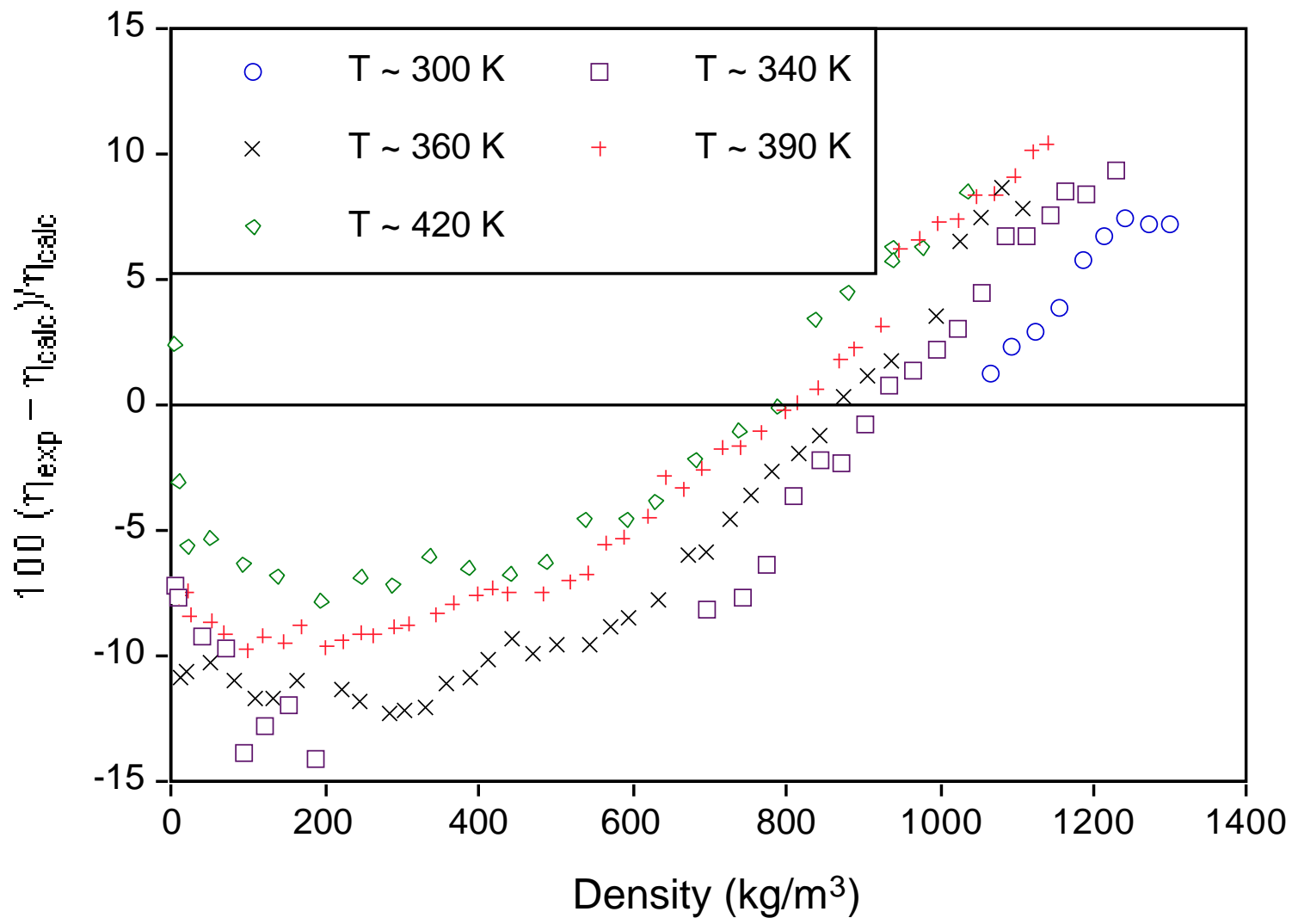

Figure 12. Percent deviations between experimental viscosities of the mixture R507A $(0.5 \mathrm{R} 143 \mathrm{a}+0.5 \mathrm{R} 125$ by mass) as measured in the torsional crystal viscometer and data calculated with the model in REFPROP. 
$100\left(\eta_{\text {lit }} / \eta_{\text {REFPROP }}-1\right)$

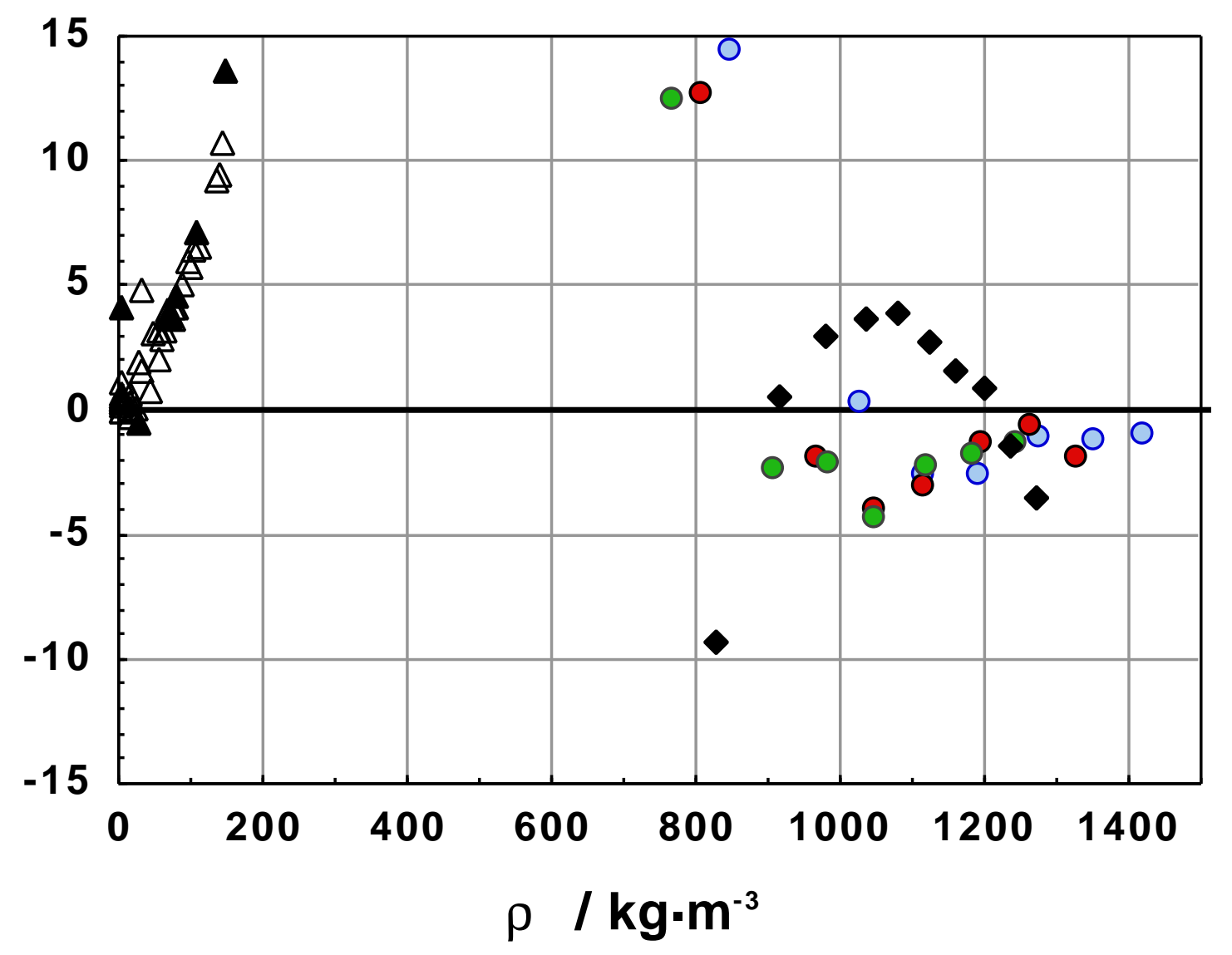

○ Heide, Schenk 1996, w(R125) $=0.752$ ○ Heide, Schenk 1996, w(R125) $=0.5$

- Heide, Schenk 1996, w(R125)=0.241 $\Delta$ Nabizadeh, Mayinger 1999

\ Nabizadeh, Mayinger 1999, sat. vap. • Fröba et al. 2001

Figure 13. Percent deviations between literature data for the viscosity of the binary system $\mathrm{R} 143 \mathrm{a}+\mathrm{R} 125$ and data calculated with the model in REFPROP. 


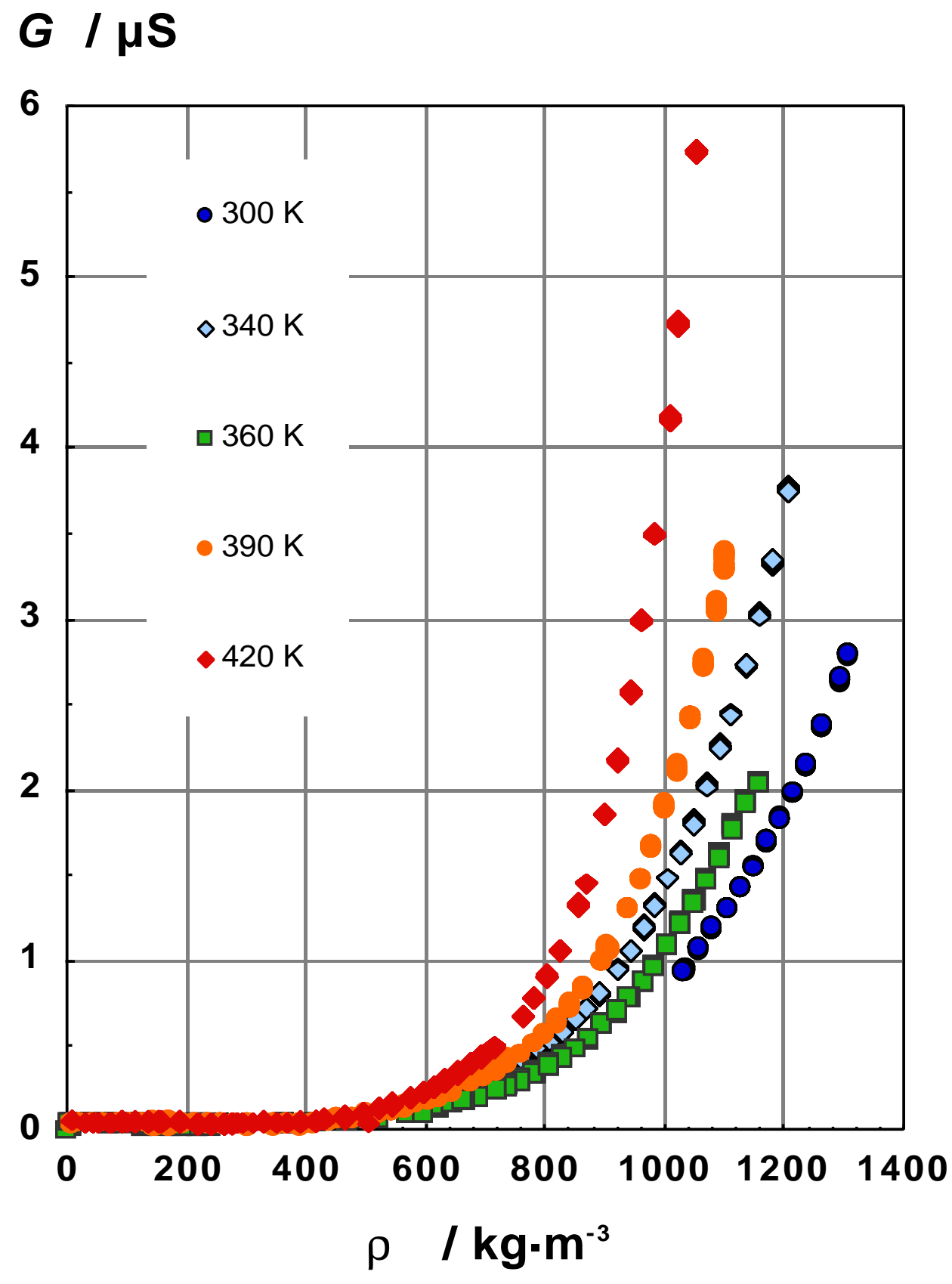

Figure 14. Conductances $G$ at $39 \mathrm{kHz}$ of the mixture R410A (0.5 R32 + $0.5 \mathrm{R} 125$ by mass) as measured in the torsional crystal viscometer. 


\section{$\eta / \mathrm{mPa} \cdot \mathbf{s}$}

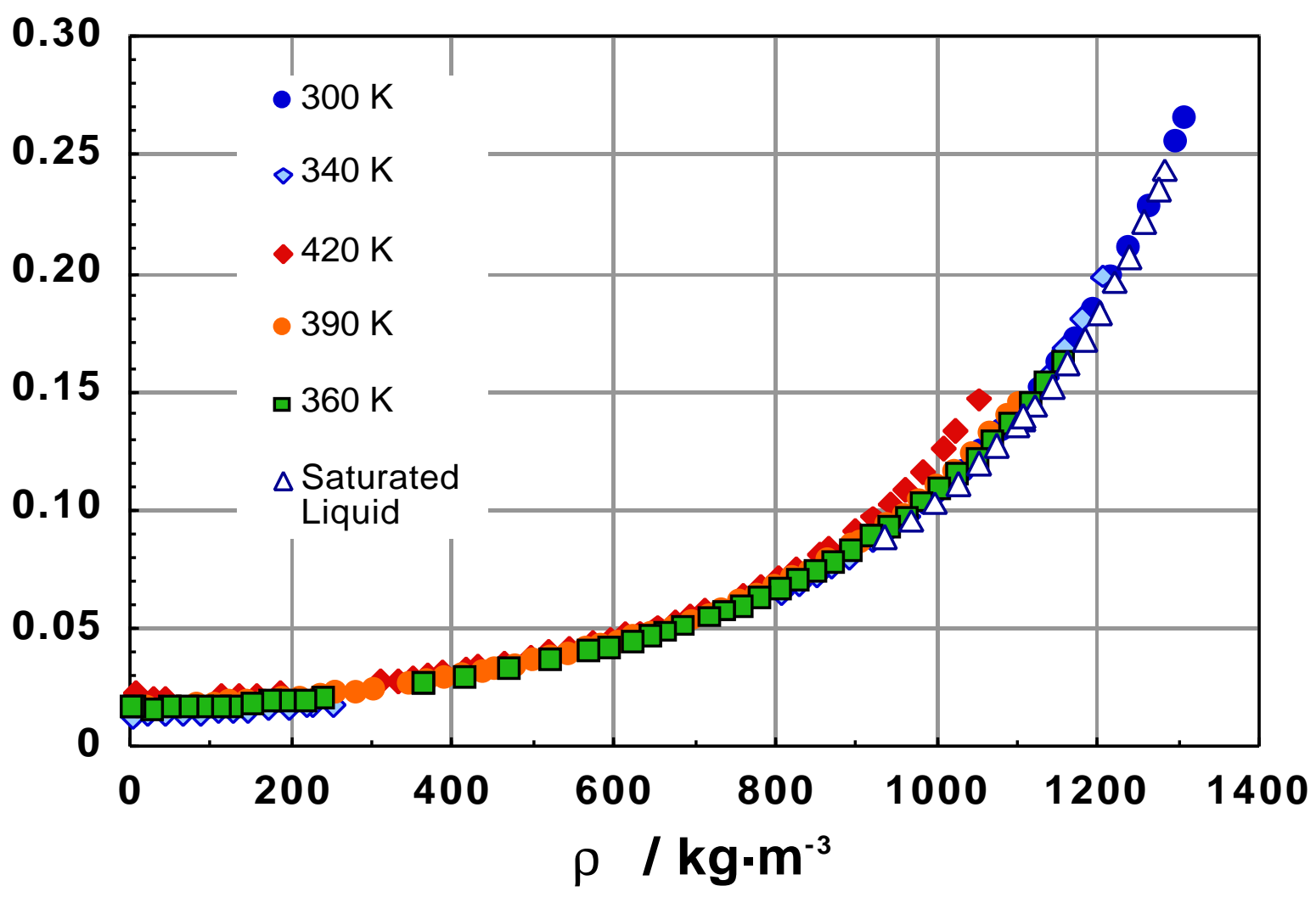

Figure 15. Density dependence of experimental viscosities of the mixture R410A $(0.5 \mathrm{R} 32+0.5$ $\mathrm{R} 125$ by mass) as measured in the torsional crystal and capillary viscometers. 


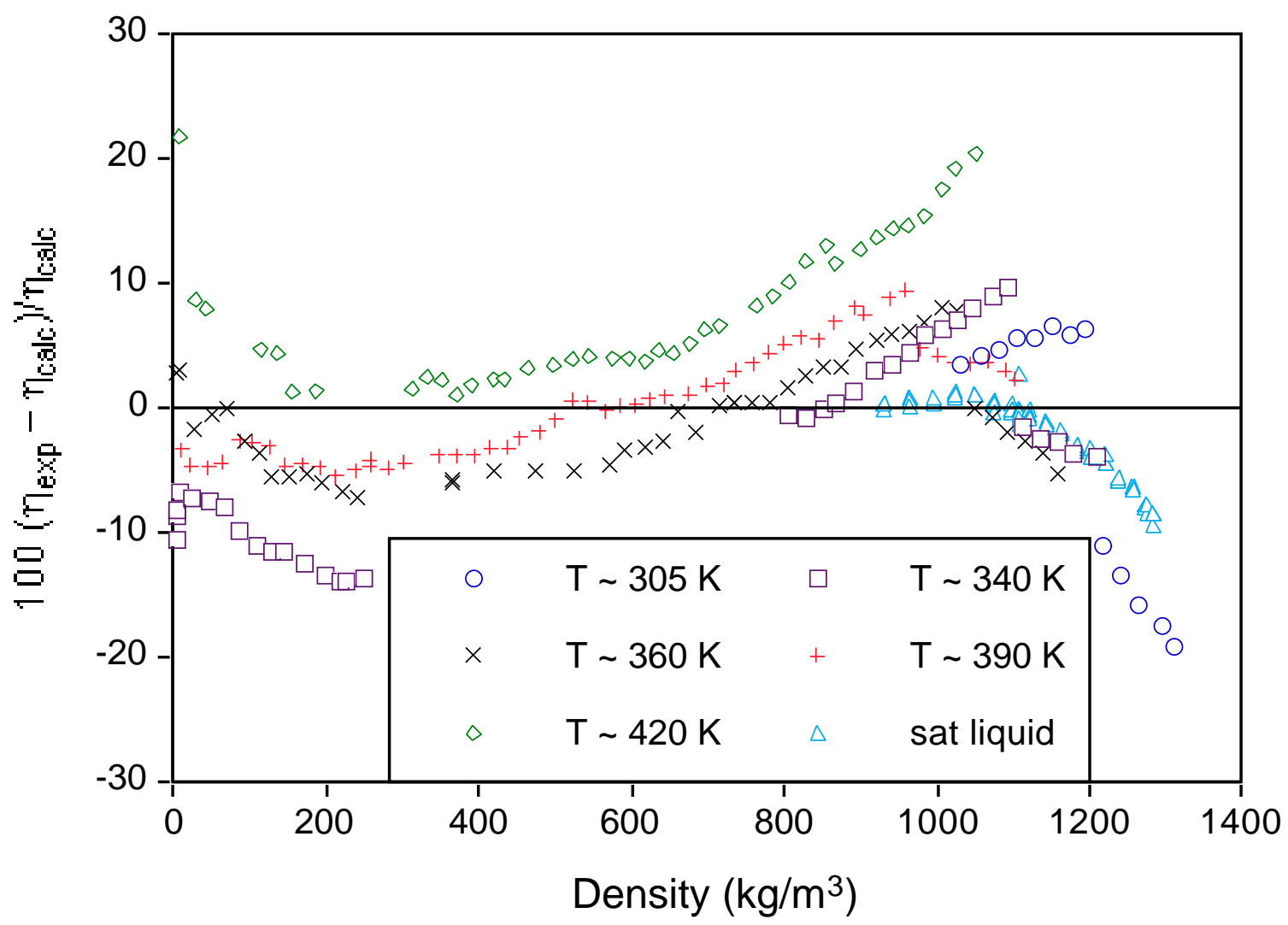

Figure 16. Percent deviations between experimental viscosities of the mixture R410A (0.5 R32 +0.5 R125 by mass) as measured in the torsional crystal viscometer and in the sealed gravitational capillary viscometer and data calculated with the model in REFPROP. 


\section{$100\left(\eta \mathrm{lit} / \eta_{\text {REFPROP }}-1\right)$}

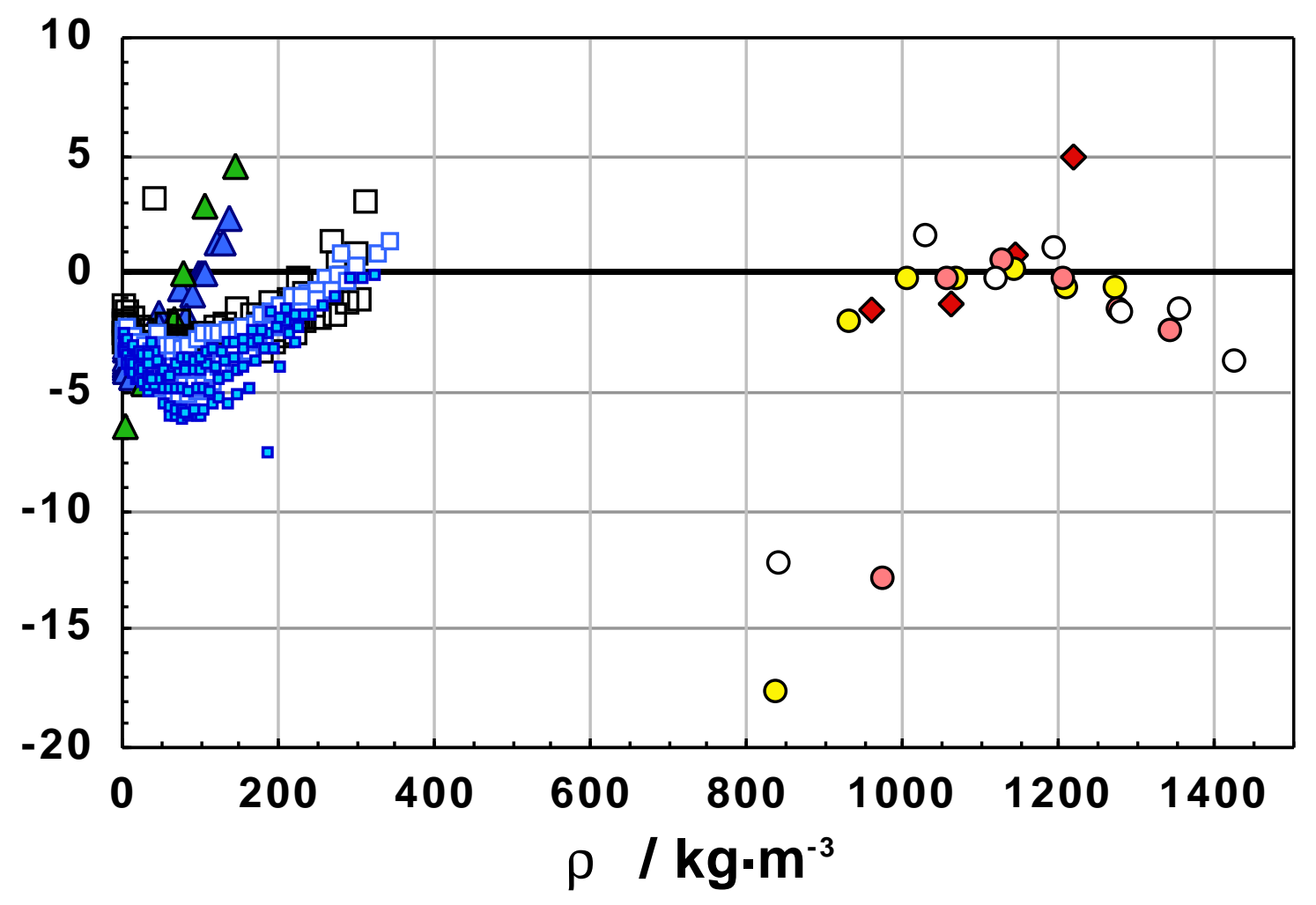

$\diamond \quad$ Bivens et al. 1993, Sat. Liq., $w(R 32)=0.6$

$\Delta \quad$ Nabizadeh, Mayinger 1999, R410A

$\Delta$ Nabizadeh, Mayinger 1999, Sat. Vap., R410A

○ Heide, Schenk 1996, Sat. Liq., $w(R 32)=0.753$

o Heide, Schenk 1996, Sat. Liq., $w(R 32)=0.51$

○ Heide, Schenk 1996, Sat. Liq., w(R32) $=0.256$

Yokoyama et al. 2001, $w(R 32)=0.1263$

$\square \quad$ Yokoyama et al. 2001, w(R32) $=0.3026$

口 Yokoyama et al. 2001, w(R32) $=0.5686$

Figure 17. Percent deviations between literature data for the viscosity of the binary system R32 + R125 and data calculated with the in REFPROP. 
Table 12. Experimental viscosities of the mixture R507A (0.5 R143a + 0.5 R125 by mass) as measured in the torsional crystal viscometer. Each line represents the average of four measurements. The precision of the measurements is indicated by the relative standard deviation of the pressure averaging, $s_{p} / p$, and of the viscosity averaging, $s_{\eta} / \eta$. Calculated densities and viscosities were obtained from REFPROP. Percent differences between experimental and calculated viscosities are listed in the column $\Delta \eta$.

\begin{tabular}{|c|c|c|c|c|c|c|c|}
\hline $\begin{array}{c}\mathrm{T} \\
(\mathrm{K})\end{array}$ & $\begin{array}{c}\mathrm{P} \\
(\mathrm{MPa})\end{array}$ & $\begin{array}{c}\eta \\
(\mathrm{mPa} \cdot \mathrm{s})\end{array}$ & $\begin{array}{l}\mathrm{S}_{\mathrm{P}} / \mathrm{P} \\
(\%)\end{array}$ & $\begin{array}{l}\mathrm{s}_{\eta} / \eta \\
(\%)\end{array}$ & $\begin{array}{c}\rho_{\text {calc }} \\
\left(\mathrm{kg} / \mathrm{m}^{3}\right)\end{array}$ & $\begin{array}{c}\eta_{\text {calc }} \\
(\mathrm{mPa} \cdot \mathrm{s})\end{array}$ & $\begin{array}{l}\Delta \eta \\
(\%)\end{array}$ \\
\hline 301.29 & 66.852 & 0.2925 & 0.03 & 0.73 & 1299.52 & 0.2731 & 7.10 \\
\hline 300.79 & 54.147 & 0.2662 & 0.02 & 0.82 & 1273.14 & 0.2485 & 7.11 \\
\hline 300.52 & 41.734 & 0.2396 & 0.01 & 0.32 & 1242.39 & 0.2232 & 7.35 \\
\hline 300.33 & 31.904 & 0.2157 & 0.07 & 0.29 & 1213.25 & 0.2022 & 6.67 \\
\hline 300.15 & 23.731 & 0.1969 & 0.07 & 0.63 & 1184.21 & 0.1861 & 5.78 \\
\hline 299.99 & 16.841 & 0.1763 & 0.09 & 0.30 & 1154.41 & 0.1699 & 3.78 \\
\hline 299.85 & 11.202 & 0.1599 & 0.12 & 0.32 & 1124.12 & 0.1556 & 2.81 \\
\hline 299.74 & 6.595 & 0.1457 & 0.13 & 0.26 & 1092.55 & 0.1425 & 2.25 \\
\hline 299.66 & 3.575 & 0.1345 & 1.99 & 0.56 & 1065.97 & 0.1329 & 1.20 \\
\hline 340.65 & 67.754 & 0.2239 & 0.00 & 0.34 & 1229.76 & 0.2050 & 9.24 \\
\hline 340.65 & 52.722 & 0.1961 & 0.01 & 0.24 & 1189.96 & 0.1809 & 8.37 \\
\hline 340.66 & 44.042 & 0.1805 & 0.01 & 0.42 & 1162.33 & 0.1664 & 8.49 \\
\hline 340.65 & 38.680 & 0.1689 & 0.01 & 0.48 & 1142.85 & 0.1570 & 7.56 \\
\hline 340.64 & 31.652 & 0.1538 & 0.00 & 0.16 & 1113.38 & 0.1441 & 6.71 \\
\hline 340.66 & 25.845 & 0.1414 & 0.04 & 0.83 & 1084.16 & 0.1326 & 6.65 \\
\hline 340.65 & 20.764 & 0.1297 & 0.02 & 0.10 & 1053.24 & 0.1242 & 4.43 \\
\hline 340.65 & 16.782 & 0.1187 & 0.00 & 0.39 & 1023.46 & 0.1153 & 3.01 \\
\hline 340.66 & 13.648 & 0.1097 & 0.01 & 0.13 & 994.57 & 0.1074 & 2.15 \\
\hline 340.66 & 11.013 & 0.1013 & 0.01 & 0.20 & 964.32 & 0.1000 & 1.31 \\
\hline 340.67 & 8.961 & 0.0941 & 0.02 & 0.10 & 934.39 & 0.0934 & 0.69 \\
\hline 340.66 & 7.292 & 0.0864 & 0.02 & 0.17 & 903.00 & 0.0871 & -0.84 \\
\hline 340.67 & 6.043 & 0.0795 & 0.00 & 0.16 & 871.73 & 0.0814 & -2.33 \\
\hline 340.65 & 5.204 & 0.0750 & 0.03 & 0.27 & 843.88 & 0.0767 & -2.22 \\
\hline 340.66 & 4.437 & 0.0686 & 0.04 & 0.14 & 807.74 & 0.0712 & -3.70 \\
\hline 340.66 & 3.986 & 0.0624 & 0.03 & 0.18 & 775.68 & 0.0667 & -6.46 \\
\hline 340.65 & 3.686 & 0.0576 & 0.05 & 0.19 & 742.02 & 0.0624 & -7.74 \\
\hline 340.66 & 3.485 & 0.0525 & 0.03 & 0.14 & 697.77 & 0.0572 & -8.20 \\
\hline 340.65 & 3.059 & 0.0169 & 0.04 & 0.14 & 188.51 & 0.0197 & -14.20 \\
\hline 340.66 & 2.767 & 0.0163 & 0.06 & 0.85 & 152.62 & 0.0185 & -11.98 \\
\hline 340.66 & 2.394 & 0.0153 & 0.09 & 1.75 & 119.18 & 0.0175 & -12.82 \\
\hline 340.68 & 2.053 & 0.0146 & 0.45 & 1.12 & 94.93 & 0.0169 & -13.86 \\
\hline 340.66 & 1.598 & 0.0146 & 0.02 & 0.57 & 68.18 & 0.0162 & -9.74 \\
\hline 340.67 & 1.002 & 0.0142 & 0.08 & 0.61 & 39.22 & 0.0156 & -9.30 \\
\hline 340.66 & 0.266 & 0.0140 & 0.00 & 0.27 & 9.54 & 0.0152 & -7.77 \\
\hline 340.66 & 0.138 & 0.0140 & 0.01 & 0.47 & 4.88 & 0.0151 & -7.28 \\
\hline
\end{tabular}


Table 12. Experimental viscosities of the mixture R507A (continued).

\begin{tabular}{|c|c|c|c|c|c|c|c|}
\hline $\begin{array}{c}\mathrm{T} \\
(\mathrm{K})\end{array}$ & $\begin{array}{c}\mathrm{P} \\
(\mathrm{MPa})\end{array}$ & $\begin{array}{c}\eta \\
(\mathrm{mPa} \cdot \mathrm{s})\end{array}$ & $\begin{array}{l}\mathrm{S}_{\mathrm{P}} / \mathrm{P} \\
(\%)\end{array}$ & $\begin{array}{l}\mathrm{s}_{\eta} / \eta \\
(\%)\end{array}$ & $\begin{array}{c}\rho_{\text {calc }} \\
\left(\mathrm{kg} / \mathrm{m}^{3}\right)\end{array}$ & $\begin{array}{c}\eta_{\text {calc }} \\
(\mathrm{mPa} \cdot \mathrm{s})\end{array}$ & $\begin{array}{l}\Delta \eta \\
(\%)\end{array}$ \\
\hline 360.73 & 41.677 & 0.1529 & 0.03 & 0.52 & 1110.22 & 0.1418 & 7.80 \\
\hline 360.64 & 35.317 & 0.1425 & 0.04 & 0.47 & 1082.21 & 0.1312 & 8.55 \\
\hline 360.59 & 30.070 & 0.1310 & 0.05 & 0.61 & 1054.95 & 0.1219 & 7.43 \\
\hline 360.57 & 25.406 & 0.1202 & 0.04 & 0.25 & 1026.16 & 0.1130 & 6.45 \\
\hline 360.58 & 21.176 & 0.1100 & 0.01 & 0.26 & 994.51 & 0.1064 & 3.41 \\
\hline 360.58 & 15.366 & 0.0944 & 0.01 & 0.19 & 936.42 & 0.0929 & 1.63 \\
\hline 360.57 & 13.105 & 0.0877 & 0.01 & 0.03 & 905.60 & 0.0867 & 1.07 \\
\hline 360.58 & 11.389 & 0.0816 & 0.01 & 0.20 & 876.48 & 0.0814 & 0.26 \\
\hline 360.58 & 9.808 & 0.0747 & 0.00 & 0.00 & 842.62 & 0.0756 & -1.28 \\
\hline 360.59 & 8.833 & 0.0701 & 0.02 & 0.16 & 816.18 & 0.0715 & -2.02 \\
\hline 360.56 & 7.799 & 0.0646 & 0.01 & 0.34 & 780.60 & 0.0664 & -2.73 \\
\hline 360.59 & 7.240 & 0.0607 & 0.01 & 0.33 & 755.45 & 0.0631 & -3.73 \\
\hline 360.59 & 6.723 & 0.0567 & 0.01 & 0.23 & 726.51 & 0.0594 & -4.58 \\
\hline 360.59 & 6.306 & 0.0526 & 0.00 & 0.16 & 696.39 & 0.0559 & -5.89 \\
\hline 360.60 & 6.035 & 0.0499 & 0.00 & 0.14 & 671.32 & 0.0531 & -6.07 \\
\hline 360.57 & 5.722 & 0.0454 & 0.00 & 0.27 & 634.13 & 0.0493 & -7.87 \\
\hline 360.58 & 5.494 & 0.0417 & 0.00 & 0.34 & 596.54 & 0.0456 & -8.62 \\
\hline 360.57 & 5.379 & 0.0396 & 0.00 & 0.14 & 572.97 & 0.0435 & -8.88 \\
\hline 360.58 & 5.262 & 0.0371 & 0.00 & 0.17 & 544.12 & 0.0410 & -9.58 \\
\hline 360.60 & 5.121 & 0.0340 & 0.00 & 0.26 & 502.76 & 0.0376 & -9.62 \\
\hline 360.58 & 5.022 & 0.0317 & 0.00 & 0.10 & 471.38 & 0.0353 & -9.98 \\
\hline 360.59 & 4.938 & 0.0301 & 0.00 & 0.17 & 443.28 & 0.0333 & -9.36 \\
\hline 360.59 & 4.839 & 0.0279 & 0.01 & 0.05 & 411.23 & 0.0311 & -10.26 \\
\hline 360.59 & 4.766 & 0.0265 & 0.00 & 0.12 & 388.95 & 0.0297 & -10.90 \\
\hline 360.59 & 4.650 & 0.0247 & 0.01 & 0.31 & 356.71 & 0.0279 & -11.22 \\
\hline 360.57 & 4.547 & 0.0233 & 0.02 & 0.14 & 331.69 & 0.0265 & -12.11 \\
\hline 360.58 & 4.421 & 0.0221 & 0.01 & 0.07 & 304.54 & 0.0251 & -12.21 \\
\hline 360.60 & 4.306 & 0.0211 & 0.01 & 0.20 & 282.88 & 0.0241 & -12.34 \\
\hline 360.59 & 4.064 & 0.0198 & 0.01 & 0.08 & 245.23 & 0.0225 & -11.91 \\
\hline 360.59 & 3.885 & 0.0191 & 0.01 & 0.24 & 222.13 & 0.0216 & -11.47 \\
\hline 360.57 & 3.294 & 0.0174 & 0.03 & 0.48 & 163.61 & 0.0196 & -11.09 \\
\hline 360.58 & 2.901 & 0.0165 & 0.04 & 0.38 & 133.88 & 0.0187 & -11.77 \\
\hline 360.58 & 2.498 & 0.0159 & 0.05 & 0.23 & 108.11 & 0.0180 & -11.78 \\
\hline 360.59 & 1.980 & 0.0153 & 0.09 & 0.79 & 79.81 & 0.0172 & -11.02 \\
\hline 360.49 & 1.331 & 0.0149 & 0.01 & 0.09 & 49.76 & 0.0166 & -10.36 \\
\hline 360.56 & 0.565 & 0.0144 & 0.01 & 0.04 & 19.57 & 0.0161 & -10.70 \\
\hline 360.59 & 0.289 & 0.0142 & 0.01 & 0.22 & 9.77 & 0.0160 & -10.96 \\
\hline
\end{tabular}


Table 12. Experimental viscosities of the mixture R507A (continued).

\begin{tabular}{|c|c|c|c|c|c|c|c|}
\hline $\begin{array}{c}\mathrm{T} \\
(\mathrm{K})\end{array}$ & $\begin{array}{c}\mathrm{P} \\
(\mathrm{MPa})\end{array}$ & $\begin{array}{c}\eta \\
(\mathrm{mPa} \cdot \mathrm{s})\end{array}$ & $\begin{array}{l}\mathrm{s}_{\mathrm{P}} / \mathrm{P} \\
(\%)\end{array}$ & $\begin{array}{l}\mathrm{s}_{\eta} / \eta \\
(\%)\end{array}$ & $\begin{array}{c}\rho_{\text {calc }} \\
\left(\mathrm{kg} / \mathrm{m}^{3}\right)\end{array}$ & $\begin{array}{c}\eta_{\text {calc }} \\
(\mathrm{mPa} \cdot \mathrm{s})\end{array}$ & $\begin{array}{l}\Delta \eta \\
(\%)\end{array}$ \\
\hline 391.03 & 66.793 & 0.1685 & 0.03 & 0.26 & 1139.92 & 0.1527 & 10.35 \\
\hline 390.84 & 60.494 & 0.1592 & 0.04 & 0.27 & 1120.44 & 0.1447 & 10.04 \\
\hline 390.77 & 53.893 & 0.1482 & 0.02 & 0.25 & 1097.45 & 0.1359 & 9.05 \\
\hline 390.78 & 47.712 & 0.1379 & 0.01 & 0.37 & 1072.94 & 0.1273 & 8.32 \\
\hline 390.78 & 42.065 & 0.1289 & 0.01 & 0.43 & 1047.40 & 0.1191 & 8.26 \\
\hline 390.77 & 37.422 & 0.1202 & 0.00 & 0.25 & 1023.40 & 0.1120 & 7.29 \\
\hline 390.78 & 33.137 & 0.1126 & 0.01 & 0.61 & 997.98 & 0.1050 & 7.16 \\
\hline 390.77 & 29.530 & 0.1053 & 0.00 & 0.10 & 973.39 & 0.0988 & 6.51 \\
\hline 390.78 & 26.357 & 0.0987 & 0.01 & 0.10 & 948.41 & 0.0930 & 6.11 \\
\hline 390.77 & 23.668 & 0.0924 & 0.01 & 0.42 & 924.03 & 0.0896 & 3.07 \\
\hline 390.77 & 20.405 & 0.0847 & 0.01 & 0.37 & 888.75 & 0.0829 & 2.17 \\
\hline 390.74 & 18.896 & 0.0809 & 0.01 & 0.08 & 869.64 & 0.0795 & 1.73 \\
\hline 390.76 & 16.983 & 0.0753 & 0.04 & 0.26 & 841.50 & 0.0748 & 0.58 \\
\hline 390.77 & 15.498 & 0.0709 & 0.02 & 0.13 & 815.83 & 0.0709 & -0.01 \\
\hline 390.77 & 14.567 & 0.0680 & 0.02 & 0.23 & 797.47 & 0.0682 & -0.34 \\
\hline 390.78 & 13.206 & 0.0632 & 0.03 & 0.15 & 766.25 & 0.0639 & -1.18 \\
\hline 390.77 & 12.251 & 0.0596 & 0.01 & 0.23 & 740.32 & 0.0606 & -1.72 \\
\hline 390.78 & 11.488 & 0.0567 & 0.01 & 0.08 & 716.14 & 0.0577 & -1.78 \\
\hline 390.77 & 10.796 & 0.0534 & 0.00 & 0.10 & 690.91 & 0.0548 & -2.65 \\
\hline 390.77 & 10.212 & 0.0504 & 0.01 & 0.18 & 666.26 & 0.0522 & -3.44 \\
\hline 390.78 & 9.742 & 0.0484 & 0.00 & 0.14 & 643.54 & 0.0499 & -2.95 \\
\hline 390.80 & 9.349 & 0.0456 & 0.00 & 0.12 & 622.07 & 0.0478 & -4.54 \\
\hline 390.77 & 8.796 & 0.0422 & 0.00 & 0.24 & 587.73 & 0.0446 & -5.40 \\
\hline 390.78 & 8.473 & 0.0402 & 0.03 & 0.14 & 564.56 & 0.0426 & -5.61 \\
\hline 390.78 & 8.179 & 0.0379 & 0.02 & 0.14 & 541.41 & 0.0407 & -6.83 \\
\hline 390.77 & 7.907 & 0.0361 & 0.02 & 0.25 & 518.20 & 0.0389 & -7.09 \\
\hline 390.78 & 7.529 & 0.0335 & 0.02 & 0.17 & 482.84 & 0.0363 & -7.56 \\
\hline 390.78 & 7.085 & 0.0307 & 0.02 & 0.24 & 437.97 & 0.0333 & -7.60 \\
\hline 390.77 & 6.891 & 0.0296 & 0.03 & 0.17 & 417.74 & 0.0320 & -7.42 \\
\hline 390.76 & 6.693 & 0.0284 & 0.03 & 0.18 & 396.96 & 0.0308 & -7.64 \\
\hline 390.77 & 6.394 & 0.0267 & 0.01 & 0.23 & 365.69 & 0.0290 & -7.98 \\
\hline 390.79 & 6.194 & 0.0256 & 0.01 & 0.18 & 345.18 & 0.0279 & -8.43 \\
\hline 390.78 & 5.821 & 0.0238 & 0.01 & 0.21 & 308.80 & 0.0261 & -8.80 \\
\hline 390.77 & 5.618 & 0.0230 & 0.00 & 0.25 & 290.08 & 0.0253 & -8.94 \\
\hline 390.76 & 5.311 & 0.0219 & 0.01 & 0.20 & 263.32 & 0.0241 & -9.23 \\
\hline 390.78 & 5.103 & 0.0213 & 0.00 & 0.21 & 246.19 & 0.0234 & -9.16 \\
\hline 390.77 & 4.787 & 0.0204 & 0.02 & 0.33 & 221.93 & 0.0225 & -9.51 \\
\hline 390.77 & 4.502 & 0.0197 & 0.01 & 0.11 & 201.62 & 0.0218 & -9.75 \\
\hline 390.78 & 3.977 & 0.0189 & 0.12 & 0.73 & 167.73 & 0.0207 & -8.90 \\
\hline 390.78 & 3.604 & 0.0182 & 0.02 & 0.34 & 146.10 & 0.0201 & -9.55 \\
\hline 390.77 & 3.095 & 0.0176 & 0.17 & 0.39 & 119.32 & 0.0194 & -9.28 \\
\hline 390.79 & 2.668 & 0.0169 & 0.17 & 0.88 & 98.89 & 0.0187 & -9.82 \\
\hline 390.76 & 1.985 & 0.0165 & 0.17 & 0.29 & 69.47 & 0.0182 & -9.17 \\
\hline 390.78 & 1.551 & 0.0163 & 0.05 & 0.11 & 52.47 & 0.0178 & -8.69 \\
\hline 390.78 & 0.773 & 0.0159 & 0.09 & 0.26 & 24.73 & 0.0174 & -8.45 \\
\hline 390.77 & 0.632 & 0.0160 & 0.01 & 0.05 & 20.03 & 0.0173 & -7.50 \\
\hline 390.79 & 0.320 & 0.0158 & 0.01 & 0.19 & 9.93 & 0.0171 & -7.71 \\
\hline 390.78 & 0.161 & 0.0158 & 0.01 & 0.15 & 4.95 & 0.0171 & -7.49 \\
\hline
\end{tabular}


Table 12. Experimental viscosities of the mixture R507A (continued).

$\begin{array}{cccccccr}\mathrm{T} & \mathrm{P} & \begin{array}{c}\eta \\ (\mathrm{mPa} \cdot \mathrm{s})\end{array} & \begin{array}{c}\mathrm{s}_{\mathrm{P}} / \mathrm{P} \\ (\%)\end{array} & \begin{array}{c}\mathrm{s}_{\eta} / \eta \\ (\%)\end{array} & \begin{array}{c}\rho_{\text {calc }} \\ \left(\mathrm{kg} / \mathrm{m}^{3}\right)\end{array} & \begin{array}{c}\eta_{\text {calc }} \\ (\mathrm{mPa} \cdot \mathrm{s})\end{array} & \begin{array}{c}\Delta \eta \\ (\%)\end{array} \\ 420.82 & 52.103 & 0.1249 & 0.00 & 0.35 & 1034.65 & 0.1152 & 8.45 \\ 420.88 & 40.738 & 0.1062 & 0.00 & 0.32 & 976.84 & 0.0999 & 6.22 \\ 420.90 & 34.770 & 0.0963 & 0.00 & 0.36 & 937.95 & 0.0911 & 5.68 \\ 420.89 & 34.756 & 0.0968 & 0.01 & 0.25 & 937.87 & 0.0911 & 6.25 \\ 420.91 & 27.811 & 0.0832 & 0.05 & 0.16 & 879.74 & 0.0796 & 4.47 \\ 420.90 & 23.853 & 0.0747 & 0.00 & 0.11 & 836.55 & 0.0723 & 3.36 \\ 420.89 & 20.410 & 0.0668 & 0.01 & 0.21 & 788.99 & 0.0669 & -0.14 \\ 420.92 & 17.528 & 0.0596 & 0.00 & 0.13 & 737.60 & 0.0602 & -1.07 \\ 420.91 & 15.087 & 0.0526 & 0.00 & 0.13 & 680.91 & 0.0538 & -2.22 \\ 420.90 & 13.329 & 0.0466 & 0.01 & 0.26 & 628.14 & 0.0485 & -3.89 \\ 420.90 & 12.333 & 0.0431 & 0.01 & 0.13 & 591.82 & 0.0452 & -4.59 \\ 420.92 & 11.104 & 0.0389 & 0.00 & 0.12 & 538.62 & 0.0408 & -4.61 \\ 420.92 & 10.076 & 0.0346 & 0.00 & 0.30 & 486.14 & 0.0369 & -6.31 \\ 420.90 & 9.291 & 0.0317 & 0.00 & 0.17 & 441.36 & 0.0340 & -6.79 \\ 420.93 & 8.392 & 0.0288 & 0.00 & 0.27 & 386.20 & 0.0308 & -6.56 \\ 420.92 & 7.574 & 0.0264 & 0.00 & 0.11 & 334.93 & 0.0282 & -6.12 \\ 420.92 & 6.793 & 0.0241 & 0.01 & 0.12 & 286.98 & 0.0260 & -7.25 \\ 420.92 & 6.098 & 0.0227 & 0.00 & 0.03 & 246.42 & 0.0244 & -6.91 \\ 420.92 & 5.122 & 0.0208 & 0.02 & 0.52 & 194.06 & 0.0226 & -7.88 \\ 420.91 & 3.907 & 0.0193 & 0.01 & 0.20 & 136.84 & 0.0207 & -6.86 \\ 420.93 & 2.840 & 0.0185 & 0.03 & 0.24 & 93.21 & 0.0198 & -6.38 \\ 420.93 & 1.609 & 0.0179 & 0.04 & 0.07 & 49.29 & 0.0189 & -5.40 \\ 420.92 & 0.731 & 0.0174 & 0.05 & 0.10 & 21.40 & 0.0184 & -5.65 \\ 420.91 & 0.333 & 0.0177 & 0.01 & 0.08 & 9.56 & 0.0183 & -3.12 \\ 420.91 & 0.090 & 0.0186 & 0.02 & 0.08 & 2.55 & 0.0181 & 2.37\end{array}$


Table 13. Experimental viscosities of the mixture R410A (0.5 R32 + 0.5 R125 by mass) as measured in the torsional crystal viscometer. (Symbols same as Table 12.)

\begin{tabular}{|c|c|c|c|c|c|c|c|}
\hline $\begin{array}{c}\mathrm{T} \\
(\mathrm{K})\end{array}$ & $\begin{array}{c}\mathrm{P} \\
(\mathrm{MPa})\end{array}$ & $\begin{array}{c}\eta \\
(\mathrm{mPa} \cdot \mathrm{s})\end{array}$ & $\begin{array}{l}\mathrm{S}_{\mathrm{P}} / \mathrm{P} \\
(\%)\end{array}$ & $\begin{array}{l}\mathrm{s}_{\eta} / \eta \\
(\%)\end{array}$ & $\begin{array}{c}\rho_{\text {calc }} \\
\left(\mathrm{kg} / \mathrm{m}^{3}\right)\end{array}$ & $\begin{array}{c}\eta_{\text {calc }} \\
(\mathrm{mPa} \cdot \mathrm{s})\end{array}$ & $\begin{array}{l}\Delta \eta \\
(\%)\end{array}$ \\
\hline 304.72 & 82.477 & 0.2653 & 0.00 & 0.62 & 1309.85 & 0.3284 & -19.23 \\
\hline 304.79 & 75.079 & 0.2552 & 0.03 & 0.45 & 1296.48 & 0.3098 & -17.63 \\
\hline 304.80 & 59.368 & 0.2280 & 0.01 & 0.53 & 1265.04 & 0.2711 & -15.91 \\
\hline 304.84 & 48.136 & 0.2104 & 0.00 & 0.67 & 1238.78 & 0.2434 & -13.57 \\
\hline 304.87 & 40.172 & 0.1986 & 0.01 & 0.42 & 1217.52 & 0.2237 & -11.21 \\
\hline 304.87 & 32.857 & 0.1842 & 0.01 & 0.48 & 1195.44 & 0.1734 & 6.25 \\
\hline 304.90 & 26.608 & 0.1723 & 0.02 & 0.38 & 1173.78 & 0.1628 & 5.85 \\
\hline 304.85 & 20.770 & 0.1623 & 0.01 & 0.35 & 1150.64 & 0.1525 & 6.42 \\
\hline 304.78 & 15.808 & 0.1512 & 0.02 & 0.29 & 1127.69 & 0.1433 & 5.54 \\
\hline 304.69 & 11.463 & 0.1419 & 0.04 & 0.40 & 1103.95 & 0.1346 & 5.45 \\
\hline 304.56 & 7.820 & 0.1326 & 0.09 & 0.00 & 1080.16 & 0.1267 & 4.63 \\
\hline 304.43 & 4.797 & 0.1243 & 0.17 & 0.11 & 1056.00 & 0.1194 & 4.05 \\
\hline 304.58 & 2.530 & 0.1167 & 0.12 & 0.28 & 1031.89 & 0.1128 & 3.46 \\
\hline 340.38 & 67.716 & 0.1986 & 0.01 & 0.22 & 1208.42 & 0.2072 & -4.12 \\
\hline 340.38 & 56.626 & 0.1799 & 0.01 & 0.33 & 1179.58 & 0.1867 & -3.68 \\
\hline 340.38 & 49.354 & 0.1681 & 0.00 & 0.39 & 1157.95 & 0.1731 & -2.93 \\
\hline 340.38 & 42.629 & 0.1562 & 0.01 & 0.21 & 1135.35 & 0.1603 & -2.58 \\
\hline 340.38 & 36.230 & 0.1453 & 0.01 & 0.37 & 1110.75 & 0.1477 & -1.66 \\
\hline 340.37 & 32.249 & 0.1384 & 0.01 & 0.33 & 1093.45 & 0.1265 & 9.43 \\
\hline 340.37 & 27.851 & 0.1303 & 0.01 & 0.26 & 1071.90 & 0.1198 & 8.76 \\
\hline 340.36 & 23.520 & 0.1217 & 0.01 & 0.22 & 1047.42 & 0.1128 & 7.89 \\
\hline 340.38 & 20.338 & 0.1147 & 0.02 & 0.40 & 1026.45 & 0.1073 & 6.93 \\
\hline 340.38 & 17.696 & 0.1087 & 0.01 & 0.19 & 1006.53 & 0.1024 & 6.10 \\
\hline 340.37 & 15.043 & 0.1027 & 0.01 & 0.25 & 983.27 & 0.0971 & 5.75 \\
\hline 340.36 & 13.208 & 0.0971 & 0.01 & 0.20 & 964.49 & 0.0931 & 4.31 \\
\hline 340.38 & 11.298 & 0.0915 & 0.02 & 0.39 & 941.36 & 0.0885 & 3.46 \\
\hline 340.40 & 9.774 & 0.0866 & 0.04 & 0.21 & 919.14 & 0.0843 & 2.77 \\
\hline 340.36 & 8.142 & 0.0802 & 0.02 & 0.33 & 889.73 & 0.0792 & 1.19 \\
\hline 340.38 & 7.200 & 0.0759 & 0.04 & 0.35 & 867.71 & 0.0757 & 0.28 \\
\hline 340.37 & 6.586 & 0.0729 & 0.03 & 0.10 & 850.35 & 0.0731 & -0.31 \\
\hline 340.37 & 5.930 & 0.0690 & 0.04 & 0.08 & 827.09 & 0.0697 & -1.04 \\
\hline 340.37 & 5.426 & 0.0661 & 0.02 & 0.11 & 803.49 & 0.0666 & -0.69 \\
\hline 340.38 & 4.398 & 0.0188 & 0.00 & 0.18 & 249.85 & 0.0218 & -13.84 \\
\hline 340.37 & 4.293 & 0.0179 & 0.01 & 0.26 & 226.34 & 0.0209 & -14.08 \\
\hline 340.36 & 4.252 & 0.0177 & 0.00 & 0.20 & 219.00 & 0.0206 & -13.94 \\
\hline 340.37 & 4.102 & 0.0171 & 0.02 & 0.39 & 196.60 & 0.0198 & -13.63 \\
\hline 340.40 & 3.884 & 0.0166 & 0.03 & 0.41 & 171.85 & 0.0190 & -12.60 \\
\hline 340.38 & 3.583 & 0.0162 & 0.03 & 0.84 & 145.79 & 0.0183 & -11.61 \\
\hline 340.39 & 3.341 & 0.0157 & 0.03 & 0.44 & 128.64 & 0.0178 & -11.57 \\
\hline 340.37 & 3.007 & 0.0154 & 0.02 & 0.46 & 108.58 & 0.0173 & -11.25 \\
\hline 340.36 & 2.586 & 0.0151 & 0.02 & 0.26 & 87.23 & 0.0168 & -9.87 \\
\hline 340.37 & 2.084 & 0.0148 & 0.00 & 0.06 & 65.63 & 0.0161 & -8.01 \\
\hline 340.38 & 1.538 & 0.0146 & 0.00 & 0.09 & 45.45 & 0.0158 & -7.51 \\
\hline 340.38 & 0.792 & 0.0143 & 0.00 & 0.07 & 21.73 & 0.0155 & -7.40 \\
\hline 340.39 & 0.287 & 0.0142 & 0.01 & 0.05 & 7.53 & 0.0153 & -6.80 \\
\hline 340.39 & 0.211 & 0.0140 & 0.00 & 0.04 & 5.50 & 0.0152 & -8.22 \\
\hline
\end{tabular}


Table 13. Experimental viscosities of the mixture R410A (continued).

\begin{tabular}{|c|c|c|c|c|c|c|c|}
\hline $\begin{array}{c}\mathrm{T} \\
(\mathrm{K})\end{array}$ & $\begin{array}{c}\mathrm{P} \\
(\mathrm{MPa})\end{array}$ & $\begin{array}{c}\eta \\
(\mathrm{mPa} \cdot \mathrm{s})\end{array}$ & $\begin{array}{l}\mathrm{s}_{\mathrm{P}} / \mathrm{P} \\
(\%)\end{array}$ & $\begin{array}{l}\mathrm{s}_{\eta} / \eta \\
(\%)\end{array}$ & $\begin{array}{c}\rho_{\text {calc }} \\
\left(\mathrm{kg} / \mathrm{m}^{3}\right)\end{array}$ & $\begin{array}{c}\eta_{\text {calc }} \\
(\mathrm{mPa} \cdot \mathrm{s})\end{array}$ & $\begin{array}{l}\Delta \eta \\
(\%)\end{array}$ \\
\hline 340.38 & 0.141 & 0.0139 & 0.00 & 0.05 & 3.66 & 0.0152 & -8.83 \\
\hline 340.38 & 0.090 & 0.0136 & 0.00 & 0.12 & 2.32 & 0.0152 & -10.71 \\
\hline 420.81 & 69.034 & 0.1460 & 0.00 & 0.47 & 1051.82 & 0.1213 & 20.37 \\
\hline 420.80 & 61.517 & 0.1337 & 0.01 & 0.64 & 1023.88 & 0.1122 & 19.10 \\
\hline 420.83 & 57.383 & 0.1258 & 0.00 & 0.17 & 1006.73 & 0.1071 & 17.44 \\
\hline 420.82 & 52.044 & 0.1157 & 0.00 & 0.28 & 982.43 & 0.1004 & 15.27 \\
\hline 420.83 & 47.959 & 0.1088 & 0.00 & 0.39 & 961.70 & 0.0951 & 14.45 \\
\hline 420.80 & 44.321 & 0.1031 & 0.00 & 0.23 & 941.41 & 0.0902 & 14.26 \\
\hline 420.80 & 40.831 & 0.0970 & 0.00 & 0.27 & 919.79 & 0.0854 & 13.53 \\
\hline 420.79 & 37.785 & 0.0913 & 0.00 & 0.18 & 898.86 & 0.0811 & 12.53 \\
\hline 420.80 & 33.742 & 0.0837 & 0.00 & 0.08 & 867.12 & 0.0751 & 11.51 \\
\hline 420.82 & 32.400 & 0.0809 & 0.01 & 0.24 & 855.31 & 0.0716 & 12.96 \\
\hline 420.80 & 29.433 & 0.0754 & 0.00 & 0.35 & 826.55 & 0.0676 & 11.59 \\
\hline 420.80 & 27.494 & 0.0712 & 0.00 & 0.19 & 805.11 & 0.0647 & 9.97 \\
\hline 420.80 & 25.772 & 0.0675 & 0.00 & 0.09 & 783.85 & 0.0620 & 8.86 \\
\hline 420.80 & 24.254 & 0.0643 & 0.01 & 0.18 & 762.96 & 0.0595 & 8.01 \\
\hline 420.85 & 21.360 & 0.0579 & 0.01 & 0.20 & 715.54 & 0.0543 & 6.55 \\
\hline 420.83 & 20.297 & 0.0555 & 0.01 & 0.24 & 695.09 & 0.0523 & 6.18 \\
\hline 420.83 & 19.407 & 0.0530 & 0.00 & 0.19 & 676.22 & 0.0504 & 5.08 \\
\hline 420.85 & 18.478 & 0.0505 & 0.00 & 0.20 & 654.54 & 0.0484 & 4.28 \\
\hline 420.74 & 17.664 & 0.0487 & 0.01 & 0.11 & 634.30 & 0.0466 & 4.44 \\
\hline 420.79 & 17.043 & 0.0468 & 0.01 & 0.01 & 616.95 & 0.0452 & 3.64 \\
\hline 420.81 & 16.368 & 0.0452 & 0.00 & 0.51 & 596.85 & 0.0435 & 3.88 \\
\hline 420.81 & 15.678 & 0.0434 & 0.00 & 0.11 & 574.73 & 0.0418 & 3.87 \\
\hline 420.82 & 14.796 & 0.0411 & 0.01 & 0.23 & 543.78 & 0.0395 & 4.03 \\
\hline 420.82 & 14.209 & 0.0394 & 0.01 & 0.15 & 521.47 & 0.0380 & 3.79 \\
\hline 420.84 & 13.620 & 0.0376 & 0.00 & 0.08 & 497.59 & 0.0364 & 3.31 \\
\hline 420.79 & 12.842 & 0.0354 & 0.00 & 0.20 & 464.33 & 0.0344 & 3.07 \\
\hline 420.82 & 12.135 & 0.0332 & 0.01 & 0.28 & 431.96 & 0.0325 & 2.18 \\
\hline 420.80 & 11.861 & 0.0325 & 0.01 & 0.17 & 419.18 & 0.0318 & 2.19 \\
\hline 420.83 & 11.243 & 0.0308 & 0.00 & 0.11 & 389.53 & 0.0303 & 1.76 \\
\hline 420.81 & 10.848 & 0.0297 & 0.01 & 0.26 & 370.52 & 0.0294 & 0.92 \\
\hline 420.87 & 10.447 & 0.0291 & 0.01 & 0.18 & 350.90 & 0.0285 & 2.05 \\
\hline 420.85 & 10.040 & 0.0283 & 0.00 & 0.16 & 331.39 & 0.0276 & 2.38 \\
\hline 420.83 & 9.630 & 0.0272 & 0.01 & 0.08 & 311.95 & 0.0268 & 1.44 \\
\hline 420.84 & 6.697 & 0.0228 & 0.00 & 0.27 & 186.64 & 0.0225 & 1.27 \\
\hline 420.81 & 5.825 & 0.0219 & 0.03 & 0.33 & 155.31 & 0.0216 & 1.10 \\
\hline 420.82 & 5.247 & 0.0217 & 0.09 & 0.78 & 135.94 & 0.0208 & 4.29 \\
\hline 420.80 & 4.524 & 0.0213 & 0.01 & 0.21 & 113.23 & 0.0203 & 4.61 \\
\hline 420.94 & 1.909 & 0.0206 & 0.01 & 0.18 & 42.60 & 0.0191 & 7.87 \\
\hline 420.83 & 1.309 & 0.0205 & 0.00 & 0.21 & 28.53 & 0.0189 & 8.49 \\
\hline 420.78 & 0.334 & 0.0226 & 0.00 & 0.28 & 7.02 & 0.0186 & 21.68 \\
\hline 390.07 & 66.399 & 0.1448 & 0.00 & 0.36 & 1103.39 & 0.1418 & 2.11 \\
\hline 390.07 & 62.029 & 0.1396 & 0.00 & 0.25 & 1089.02 & 0.1357 & 2.85 \\
\hline 390.06 & 55.814 & 0.1315 & 0.00 & 0.23 & 1066.65 & 0.1270 & 3.59 \\
\hline 390.07 & 50.409 & 0.1232 & 0.00 & 0.35 & 1044.86 & 0.1192 & 3.42 \\
\hline
\end{tabular}


Table 13. Experimental viscosities of the mixture R410A (continued).

\begin{tabular}{|c|c|c|c|c|c|c|c|}
\hline $\begin{array}{c}\mathrm{T} \\
(\mathrm{K})\end{array}$ & $\begin{array}{c}\mathrm{P} \\
(\mathrm{MPa})\end{array}$ & $\begin{array}{c}\eta \\
(\mathrm{mPa} \cdot \mathrm{s})\end{array}$ & $\begin{array}{l}\mathrm{s}_{\mathrm{P}} / \mathrm{P} \\
(\%)\end{array}$ & $\begin{array}{l}\mathrm{s}_{\eta} / \eta \\
(\%)\end{array}$ & $\begin{array}{c}\rho_{\text {calc }} \\
\left(\mathrm{kg} / \mathrm{m}^{3}\right)\end{array}$ & $\begin{array}{c}\eta_{\text {calc }} \\
(\mathrm{mPa} \cdot \mathrm{s})\end{array}$ & $\begin{array}{l}\Delta \eta \\
(\%)\end{array}$ \\
\hline 390.07 & 45.592 & 0.1159 & 0.01 & 0.49 & 1023.19 & 0.1120 & 3.42 \\
\hline 390.05 & 41.324 & 0.1098 & 0.00 & 0.18 & 1001.77 & 0.1055 & 4.03 \\
\hline 390.06 & 37.358 & 0.1038 & 0.00 & 0.27 & 979.33 & 0.0992 & 4.56 \\
\hline 390.05 & 34.150 & 0.0982 & 0.01 & 0.23 & 959.03 & 0.0898 & 9.27 \\
\hline 390.06 & 31.118 & 0.0931 & 0.01 & 0.09 & 937.46 & 0.0857 & 8.64 \\
\hline 390.06 & 27.281 & 0.0858 & 0.00 & 0.16 & 905.84 & 0.0800 & 7.31 \\
\hline 390.04 & 25.964 & 0.0841 & 0.00 & 0.34 & 893.59 & 0.0779 & 7.96 \\
\hline 390.06 & 23.307 & 0.0784 & 0.01 & 0.84 & 865.60 & 0.0735 & 6.72 \\
\hline 390.07 & 21.577 & 0.0741 & 0.00 & 0.54 & 844.51 & 0.0703 & 5.35 \\
\hline 390.05 & 19.956 & 0.0709 & 0.01 & 0.00 & 822.11 & 0.0672 & 5.50 \\
\hline 390.06 & 18.621 & 0.0676 & 0.01 & 0.16 & 800.90 & 0.0644 & 4.98 \\
\hline 390.05 & 17.481 & 0.0644 & 0.01 & 0.14 & 780.40 & 0.0618 & 4.19 \\
\hline 390.08 & 16.424 & 0.0612 & 0.00 & 0.13 & 758.56 & 0.0592 & 3.40 \\
\hline 390.07 & 15.465 & 0.0582 & 0.00 & 0.19 & 736.12 & 0.0566 & 2.77 \\
\hline 390.04 & 14.865 & 0.0559 & 0.01 & 0.28 & 720.51 & 0.0549 & 1.77 \\
\hline 390.06 & 14.134 & 0.0535 & 0.01 & 0.29 & 698.95 & 0.0527 & 1.59 \\
\hline 390.04 & 13.459 & 0.0509 & 0.01 & 0.16 & 676.66 & 0.0505 & 0.92 \\
\hline 390.05 & 12.637 & 0.0478 & 0.00 & 0.38 & 644.96 & 0.0475 & 0.75 \\
\hline 390.05 & 12.150 & 0.0458 & 0.01 & 0.20 & 623.44 & 0.0456 & 0.58 \\
\hline 390.04 & 11.768 & 0.0440 & 0.00 & 0.15 & 604.90 & 0.0440 & 0.09 \\
\hline 390.06 & 11.388 & 0.0423 & 0.00 & 0.13 & 584.47 & 0.0423 & -0.01 \\
\hline 390.04 & 11.092 & 0.0408 & 0.01 & 0.02 & 567.54 & 0.0410 & -0.45 \\
\hline 390.05 & 10.704 & 0.0393 & 0.01 & 0.16 & 543.14 & 0.0392 & 0.33 \\
\hline 390.06 & 10.407 & 0.0379 & 0.00 & 0.13 & 522.94 & 0.0377 & 0.43 \\
\hline 390.04 & 10.109 & 0.0359 & 0.00 & 0.06 & 501.63 & 0.0363 & -1.08 \\
\hline 390.07 & 9.810 & 0.0341 & 0.00 & 0.18 & 478.60 & 0.0348 & -2.02 \\
\hline 390.05 & 9.508 & 0.0324 & 0.00 & 0.20 & 454.73 & 0.0333 & -2.52 \\
\hline 390.06 & 9.307 & 0.0312 & 0.00 & 0.01 & 438.22 & 0.0323 & -3.32 \\
\hline 390.05 & 9.026 & 0.0299 & 0.01 & 0.10 & 414.98 & 0.0310 & -3.42 \\
\hline 390.04 & 8.760 & 0.0286 & 0.00 & 0.23 & 392.87 & 0.0298 & -3.91 \\
\hline 390.06 & 8.497 & 0.0275 & 0.00 & 0.24 & 370.97 & 0.0287 & -3.98 \\
\hline 390.04 & 8.209 & 0.0264 & 0.01 & 0.15 & 347.75 & 0.0275 & -4.01 \\
\hline 390.05 & 7.621 & 0.0244 & 0.01 & 0.07 & 302.61 & 0.0255 & -4.55 \\
\hline 390.06 & 7.316 & 0.0234 & 0.02 & 0.18 & 280.87 & 0.0246 & -5.08 \\
\hline 390.06 & 6.966 & 0.0227 & 0.00 & 0.16 & 257.51 & 0.0237 & -4.36 \\
\hline 390.05 & 6.965 & 0.0226 & 0.01 & 0.20 & 257.47 & 0.0237 & -4.78 \\
\hline 390.05 & 6.644 & 0.0218 & 0.01 & 0.19 & 237.46 & 0.0230 & -5.20 \\
\hline 390.06 & 6.222 & 0.0209 & 0.00 & 0.16 & 213.09 & 0.0222 & -5.64 \\
\hline 390.06 & 5.810 & 0.0204 & 0.01 & 0.12 & 191.28 & 0.0215 & -4.91 \\
\hline 390.06 & 5.345 & 0.0199 & 0.02 & 0.19 & 168.74 & 0.0208 & -4.73 \\
\hline 390.05 & 4.845 & 0.0193 & 0.03 & 0.73 & 146.63 & 0.0202 & -4.86 \\
\hline 390.06 & 4.319 & 0.0191 & 0.09 & 0.35 & 125.38 & 0.0197 & -3.28 \\
\hline 390.06 & 3.757 & 0.0184 & 0.03 & 0.13 & 104.64 & 0.0189 & -2.87 \\
\hline 390.07 & 3.195 & 0.0181 & 0.14 & 1.66 & 85.62 & 0.0186 & -2.75 \\
\hline 390.07 & 2.449 & 0.0173 & 0.04 & 0.14 & 62.58 & 0.0182 & -4.60 \\
\hline 390.06 & 1.850 & 0.0170 & 0.00 & 0.11 & 45.63 & 0.0179 & -4.91 \\
\hline 390.06 & 0.923 & 0.0167 & 0.01 & 0.05 & 21.64 & 0.0176 & -4.82 \\
\hline 390.07 & 0.458 & 0.0168 & 0.00 & 0.14 & 10.48 & 0.0174 & -3.53 \\
\hline
\end{tabular}


Table 13. Experimental viscosities of the mixture R410A (continued).

\begin{tabular}{|c|c|c|c|c|c|c|c|}
\hline $\begin{array}{c}\mathrm{T} \\
(\mathrm{K})\end{array}$ & $\begin{array}{c}\mathrm{P} \\
(\mathrm{MPa})\end{array}$ & $\begin{array}{c}\eta \\
(\mathrm{mPa} \cdot \mathrm{s})\end{array}$ & $\begin{array}{l}\mathrm{S}_{\mathrm{P}} / \mathrm{P} \\
(\%)\end{array}$ & $\begin{array}{l}\mathrm{s}_{\eta} / \eta \\
(\%)\end{array}$ & $\begin{array}{c}\rho_{\text {calc }} \\
\left(\mathrm{kg} / \mathrm{m}^{3}\right)\end{array}$ & $\begin{array}{c}\eta_{\text {calc }} \\
(\mathrm{mPa} \cdot \mathrm{s})\end{array}$ & $\begin{array}{l}\Delta \eta \\
(\%)\end{array}$ \\
\hline 360.70 & 64.452 & 0.1615 & 0.01 & 0.30 & 1157.80 & 0.1708 & -5.41 \\
\hline 360.69 & 57.805 & 0.1538 & 0.01 & 0.17 & 1138.15 & 0.1600 & -3.90 \\
\hline 360.69 & 51.370 & 0.1451 & 0.01 & 0.20 & 1116.95 & 0.1494 & -2.87 \\
\hline 360.70 & 44.930 & 0.1355 & 0.00 & 0.43 & 1093.00 & 0.1385 & -2.14 \\
\hline 360.71 & 39.975 & 0.1286 & 0.00 & 0.22 & 1072.14 & 0.1298 & -0.93 \\
\hline 360.69 & 35.472 & 0.1215 & 0.01 & 0.31 & 1050.88 & 0.1217 & -0.19 \\
\hline 360.71 & 31.282 & 0.1145 & 0.01 & 0.35 & 1028.32 & 0.1065 & 7.57 \\
\hline 360.71 & 27.730 & 0.1090 & 0.01 & 0.20 & 1006.56 & 0.1012 & 7.77 \\
\hline 360.71 & 24.575 & 0.1026 & 0.02 & 0.13 & 984.47 & 0.0962 & 6.74 \\
\hline 360.70 & 21.881 & 0.0972 & 0.01 & 0.07 & 962.88 & 0.0916 & 6.07 \\
\hline 360.71 & 19.499 & 0.0922 & 0.01 & 0.20 & 940.82 & 0.0872 & 5.65 \\
\hline 360.68 & 17.542 & 0.0878 & 0.01 & 0.16 & 920.05 & 0.0834 & 5.27 \\
\hline 360.69 & 15.641 & 0.0829 & 0.02 & 0.13 & 896.33 & 0.0792 & 4.57 \\
\hline 360.69 & 14.110 & 0.0780 & 0.01 & 0.46 & 873.81 & 0.0756 & 3.17 \\
\hline 360.70 & 12.857 & 0.0745 & 0.01 & 0.34 & 852.01 & 0.0722 & 3.11 \\
\hline 360.71 & 11.747 & 0.0705 & 0.02 & 0.22 & 829.04 & 0.0689 & 2.30 \\
\hline 360.70 & 10.811 & 0.0667 & 0.01 & 0.17 & 805.90 & 0.0658 & 1.45 \\
\hline 360.71 & 10.003 & 0.0627 & 0.03 & 0.31 & 781.48 & 0.0626 & 0.20 \\
\hline 360.69 & 9.338 & 0.0599 & 0.01 & 0.25 & 757.12 & 0.0597 & 0.37 \\
\hline 360.70 & 8.858 & 0.0573 & 0.02 & 0.19 & 735.50 & 0.0572 & 0.25 \\
\hline 360.70 & 8.857 & 0.0573 & 0.00 & 0.08 & 735.45 & 0.0572 & 0.27 \\
\hline 360.71 & 8.483 & 0.0550 & 0.01 & 0.06 & 715.33 & 0.0550 & -0.01 \\
\hline 360.71 & 8.020 & 0.0507 & 0.01 & 0.10 & 684.71 & 0.0518 & -2.09 \\
\hline 360.70 & 7.735 & 0.0492 & 0.01 & 0.08 & 661.19 & 0.0495 & -0.53 \\
\hline 360.69 & 7.545 & 0.0464 & 0.00 & 0.13 & 642.55 & 0.0477 & -2.77 \\
\hline 360.71 & 7.357 & 0.0442 & 0.00 & 0.16 & 620.22 & 0.0457 & -3.34 \\
\hline 360.69 & 7.162 & 0.0418 & 0.01 & 0.23 & 593.08 & 0.0433 & -3.52 \\
\hline 360.70 & 7.027 & 0.0394 & 0.00 & 0.26 & 569.93 & 0.0414 & -4.83 \\
\hline 360.70 & 6.804 & 0.0358 & 0.00 & 0.23 & 523.24 & 0.0378 & -5.21 \\
\hline 360.69 & 6.604 & 0.0324 & 0.00 & 0.52 & 472.60 & 0.0342 & -5.10 \\
\hline 360.69 & 6.403 & 0.0291 & 0.00 & 0.16 & 418.94 & 0.0307 & -5.29 \\
\hline 360.71 & 6.185 & 0.0261 & 0.00 & 0.19 & 366.42 & 0.0278 & -5.95 \\
\hline 360.71 & 6.181 & 0.0260 & 0.01 & 0.18 & 365.56 & 0.0277 & -6.20 \\
\hline 360.70 & 5.375 & 0.0206 & 0.01 & 0.21 & 242.77 & 0.0222 & -7.27 \\
\hline 360.71 & 5.145 & 0.0199 & 0.02 & 0.51 & 219.90 & 0.0214 & -6.85 \\
\hline 360.71 & 4.840 & 0.0192 & 0.04 & 0.17 & 194.13 & 0.0205 & -6.24 \\
\hline 360.70 & 4.581 & 0.0189 & 0.04 & 0.63 & 175.26 & 0.0199 & -5.40 \\
\hline 360.69 & 4.232 & 0.0182 & 0.03 & 0.44 & 153.01 & 0.0193 & -5.80 \\
\hline 360.71 & 3.769 & 0.0176 & 0.01 & 0.25 & 127.63 & 0.0186 & -5.61 \\
\hline 360.70 & 3.464 & 0.0176 & 0.05 & 0.21 & 112.91 & 0.0182 & -3.72 \\
\hline 360.70 & 2.976 & 0.0173 & 0.04 & 0.81 & 91.79 & 0.0178 & -2.77 \\
\hline 360.71 & 2.446 & 0.0171 & 0.19 & 0.40 & 71.53 & 0.0171 & -0.25 \\
\hline 360.71 & 1.836 & 0.0166 & 0.00 & 0.29 & 50.84 & 0.0168 & -0.74 \\
\hline 360.70 & 1.102 & 0.0161 & 0.01 & 0.17 & 28.79 & 0.0164 & -1.86 \\
\hline 360.70 & 0.299 & 0.0166 & 0.01 & 0.14 & 7.38 & 0.0161 & 2.88 \\
\hline 360.71 & 0.149 & 0.0165 & 0.01 & 0.14 & 3.64 & 0.0161 & 2.54 \\
\hline
\end{tabular}


Table 14. Saturated-liquid viscosities the for mixture R410A (0.5 R32 + 0.5 R125 by mass) measured in the sealed gravitational capillary viscometer. (Data reported in order of measurement.)

\begin{tabular}{|c|c|c|c|c|c|}
\hline $\begin{array}{c}\mathrm{T} \\
(\mathrm{K}) \\
\text { measured }\end{array}$ & $\begin{array}{c}\rho_{\mathrm{liq}} \\
\left(\mathrm{kg} / \mathrm{m}^{3}\right) \\
\text { calculated }\end{array}$ & $\begin{array}{c}\rho_{\text {vap }} \\
\left(\mathrm{kg} / \mathrm{m}^{3}\right) \\
\text { calculated }\end{array}$ & $\begin{array}{c}\eta_{\text {liq }} \\
(\mathrm{mPa} \cdot \mathrm{s}) \\
\text { measured }\end{array}$ & $\begin{array}{c}\eta_{\text {liq }} \\
(\mathrm{mPa} \cdot \mathrm{s}) \\
\text { calculated }\end{array}$ & $\begin{array}{l}\Delta \eta \\
(\%)\end{array}$ \\
\hline 287.99 & 1107.6 & 47.85 & 0.1389 & 0.1393 & -0.28 \\
\hline 287.98 & 1107.7 & 47.84 & 0.1373 & 0.1393 & -1.44 \\
\hline 287.98 & 1107.7 & 47.84 & 0.1387 & 0.1393 & -0.44 \\
\hline 287.98 & 1107.7 & 47.84 & 0.1381 & 0.1393 & -0.87 \\
\hline 242.38 & 1282.6 & 10.03 & 0.2440 & 0.2667 & -8.53 \\
\hline 242.38 & 1282.6 & 10.03 & 0.2437 & 0.2667 & -8.64 \\
\hline 242.38 & 1282.6 & 10.03 & 0.2415 & 0.2667 & -9.46 \\
\hline 242.38 & 1282.6 & 10.03 & 0.2440 & 0.2667 & -8.53 \\
\hline 245.00 & 1273.7 & 11.12 & 0.2338 & 0.2560 & -8.67 \\
\hline 245.06 & 1273.5 & 11.15 & 0.2357 & 0.2557 & -7.84 \\
\hline 245.03 & 1273.6 & 11.14 & 0.2350 & 0.2559 & -8.16 \\
\hline 245.01 & 1273.7 & 11.13 & 0.2361 & 0.2559 & -7.76 \\
\hline 250.07 & 1256.2 & 13.50 & 0.2218 & 0.2368 & -6.34 \\
\hline 250.00 & 1256.5 & 13.46 & 0.2211 & 0.2371 & -6.74 \\
\hline 250.09 & 1256.1 & 13.51 & 0.2214 & 0.2368 & -6.48 \\
\hline 250.01 & 1256.4 & 13.47 & 0.2216 & 0.2370 & -6.51 \\
\hline 255.09 & 1238.5 & 16.23 & 0.2067 & 0.2197 & -5.93 \\
\hline 255.12 & 1238.4 & 16.25 & 0.2073 & 0.2196 & -5.62 \\
\hline 255.08 & 1238.5 & 16.23 & 0.2068 & 0.2198 & -5.90 \\
\hline 255.10 & 1238.5 & 16.24 & 0.2073 & 0.2197 & -5.65 \\
\hline 260.08 & 1220.4 & 19.38 & 0.1952 & 0.2044 & -4.48 \\
\hline 260.08 & 1220.4 & 19.38 & 0.1953 & 0.2044 & -4.43 \\
\hline 260.08 & 1220.4 & 19.38 & 0.1951 & 0.2044 & -4.53 \\
\hline 260.08 & 1220.4 & 19.38 & 0.1965 & 0.2044 & -3.85 \\
\hline 265.16 & 1201.5 & 23.08 & 0.1825 & 0.1901 & -4.02 \\
\hline 265.15 & 1201.5 & 23.07 & 0.1837 & 0.1902 & -3.40 \\
\hline 265.16 & 1201.5 & 23.08 & 0.1823 & 0.1901 & -4.12 \\
\hline 265.15 & 1201.5 & 23.07 & 0.1825 & 0.1902 & -4.03 \\
\hline 270.08 & 1182.6 & 27.19 & 0.1712 & 0.1775 & -3.57 \\
\hline 270.08 & 1182.6 & 27.19 & 0.1720 & 0.1775 & -3.12 \\
\hline 270.08 & 1182.6 & 27.19 & 0.1718 & 0.1775 & -3.24 \\
\hline 270.08 & 1182.6 & 27.19 & 0.1720 & 0.1775 & -3.12 \\
\hline 275.08 & 1162.7 & 31.98 & 0.1622 & 0.1658 & -2.16 \\
\hline 275.08 & 1162.7 & 31.98 & 0.1621 & 0.1658 & -2.22 \\
\hline 275.07 & 1162.7 & 31.97 & 0.1621 & 0.1658 & -2.24 \\
\hline 275.07 & 1162.7 & 31.97 & 0.1625 & 0.1658 & -2.00 \\
\hline 280.04 & 1142.2 & 37.43 & 0.1525 & 0.1550 & -1.62 \\
\hline 280.03 & 1142.3 & 37.41 & 0.1531 & 0.1550 & -1.24 \\
\hline 280.04 & 1142.2 & 37.43 & 0.1523 & 0.1550 & -1.75 \\
\hline 280.03 & 1142.3 & 37.41 & 0.1530 & 0.1550 & -1.31 \\
\hline 285.13 & 1120.4 & 43.84 & 0.1444 & 0.1447 & -0.24 \\
\hline 285.11 & 1120.4 & 43.81 & 0.1437 & 0.1448 & -0.75 \\
\hline 285.12 & 1120.4 & 43.82 & 0.1437 & 0.1448 & -0.74 \\
\hline 285.12 & 1120.4 & 43.82 & 0.1435 & 0.1448 & -0.87 \\
\hline
\end{tabular}


Table 14. Saturated-liquid viscosities for R410A (continued).

\begin{tabular}{|c|c|c|c|c|c|}
\hline $\begin{array}{c}\mathrm{T} \\
(\mathrm{K}) \\
\text { measured }\end{array}$ & $\begin{array}{c}\rho_{\text {liq }} \\
\left(\mathrm{kg} / \mathrm{m}^{3}\right) \\
\text { calculated }\end{array}$ & $\begin{array}{c}\rho_{\text {vap }} \\
\left(\mathrm{kg} / \mathrm{m}^{3}\right) \\
\text { calculated }\end{array}$ & $\begin{array}{c}\eta_{\text {liq }} \\
(\mathrm{mPa} \cdot \mathrm{s}) \\
\text { measured }\end{array}$ & $\begin{array}{c}\eta_{\text {liq }} \\
(\mathrm{mPa} \cdot \mathrm{s}) \\
\text { calculated }\end{array}$ & $\begin{array}{l}\Delta \eta \\
(\%)\end{array}$ \\
\hline 288.06 & 1107.3 & 47.95 & 0.1428 & 0.1392 & 2.62 \\
\hline 288.06 & 1107.3 & 47.95 & 0.1387 & 0.1392 & -0.33 \\
\hline 288.06 & 1107.3 & 47.95 & 0.1388 & 0.1392 & -0.26 \\
\hline 288.06 & 1107.3 & 47.95 & 0.1382 & 0.1392 & -0.69 \\
\hline 290.11 & 1098.0 & 51.04 & 0.1356 & 0.1354 & 0.17 \\
\hline 290.10 & 1098.0 & 51.02 & 0.1349 & 0.1354 & -0.36 \\
\hline 290.11 & 1098.0 & 51.04 & 0.1351 & 0.1354 & -0.20 \\
\hline 290.10 & 1098.0 & 51.02 & 0.1350 & 0.1354 & -0.29 \\
\hline 295.13 & 1074.3 & 59.39 & 0.1269 & 0.1265 & 0.31 \\
\hline 295.14 & 1074.2 & 59.41 & 0.1270 & 0.1265 & 0.41 \\
\hline 295.13 & 1074.3 & 59.39 & 0.1267 & 0.1265 & 0.16 \\
\hline 295.13 & 1074.3 & 59.39 & 0.1260 & 0.1265 & -0.40 \\
\hline 300.13 & 1049.3 & 68.98 & 0.1193 & 0.1182 & 0.97 \\
\hline 300.13 & 1049.3 & 68.98 & 0.1194 & 0.1182 & 1.05 \\
\hline 300.13 & 1049.3 & 68.98 & 0.1192 & 0.1182 & 0.88 \\
\hline 300.13 & 1049.3 & 68.98 & 0.1192 & 0.1182 & 0.88 \\
\hline 305.06 & 1023.2 & 79.94 & 0.1112 & 0.1103 & 0.80 \\
\hline 305.06 & 1023.2 & 79.94 & 0.1115 & 0.1103 & 1.07 \\
\hline 305.05 & 1023.3 & 79.91 & 0.1114 & 0.1103 & 0.96 \\
\hline 305.06 & 1023.2 & 79.94 & 0.1116 & 0.1103 & 1.16 \\
\hline 310.08 & 994.8 & 92.95 & 0.1030 & 0.1027 & 0.32 \\
\hline 310.08 & 994.8 & 92.95 & 0.1032 & 0.1027 & 0.51 \\
\hline 310.07 & 994.8 & 92.92 & 0.1032 & 0.1027 & 0.50 \\
\hline 310.08 & 994.8 & 92.95 & 0.1034 & 0.1027 & 0.70 \\
\hline 315.18 & 963.5 & 108.58 & 0.0958 & 0.0952 & 0.63 \\
\hline 315.18 & 963.5 & 108.58 & 0.0960 & 0.0952 & 0.84 \\
\hline 315.17 & 963.5 & 108.55 & 0.0957 & 0.0952 & 0.50 \\
\hline 315.17 & 963.5 & 108.55 & 0.0953 & 0.0952 & 0.09 \\
\hline 320.07 & 930.5 & 126.53 & 0.0884 & 0.0881 & 0.31 \\
\hline 320.08 & 930.4 & 126.57 & 0.0882 & 0.0881 & 0.15 \\
\hline 320.08 & 930.4 & 126.57 & 0.0880 & 0.0881 & -0.14 \\
\hline 320.09 & 930.3 & 126.61 & 0.0883 & 0.0881 & 0.20 \\
\hline 288.05 & 1107.4 & 47.94 & 0.1390 & 0.1392 & -0.13 \\
\hline 288.02 & 1107.5 & 47.90 & 0.1384 & 0.1392 & -0.60 \\
\hline 288.04 & 1107.4 & 47.92 & 0.1388 & 0.1392 & -0.28 \\
\hline 288.04 & 1107.4 & 47.92 & 0.1383 & 0.1392 & -0.64 \\
\hline
\end{tabular}




\section{CONCLUSIONS}

Measurements of thermophysical properties have been carried out to address some of the key data gaps for the properties of concern vis a vis the performance of blends operating at extreme conditions. These data have been used to develop new models for the thermodynamic properties for R125 and blends of the HFCs. The new equation of state for R125 is believed to be the most accurate and comprehensive formulation of the properties for that fluid. Likewise, the mixture model developed in this work is the new state of the art for the thermodynamic properties of HFC blends. These models are being incorporated into version 7 of the NIST REFPROP database to be released in the summer of 2002.

Measurements of the viscosity and thermal conductivity of two HFC blends were completed. These data were used to assess the current models for the transport properties of mixtures. These comparisons reveal that further development and refinement of the transport models is warranted. 


\section{REFERENCES}

Abbott, A.P., Eardley, C.A. and Tooth, R. (1999). Relative permittivity measurements of 1,1,1,2tetrafluoroethane (HFC 134a), pentafluoroethane (HFC 125), and difluoromethane (HFC 32). J. Chem. Eng. Data 44: 112-115.

Barão, M.T., Nieto de Castro, C.A. and Mardolcar, U.V. (1997). Molecular properties of alternative refrigerants derived from dielectric-constant measurements. Int. J. Thermophysics 18: 419-438.

Bivens, D.B., Yokozeki, A., Geller, V.Z. and Paulaitis, M.E. (1993). Transport properties and heat transfer of alternatives for R502 and R22. ASHRAE/NIST Refrigerants Conference, August 19-20, Gaithersburg, MD, ASHRAE, 73-84.

Diller, D.E. and Frederick, N.V. (1989). Torsional piezoelectric crystal viscometer for compressed gases and liquids. Int. J. Thermophysics 10: 145-157.

Fröba, A.P., Will, S. and Leipertz, A. (2001). Thermophysical properties of binary and ternary fluid mixtures from dynamic light scattering. Int. J. Thermophysics 22: 1349-1368.

Heide, R. and Schenk, J. (1996). Bestimmung der Transportgrößen von HFKW, Heft 1 Viskosität und Oberflächenspannun, Frankfurt am Main, Germany: Forschungsrat Kältetechnik e. V.

Klein, S.A., McLinden, M.O. and Laesecke, A. (1997). An improved extended corresponding states method for estimation of viscosity of pure refrigerants and mixtures. Int. J. Refrigeration 20: 208-217.

Laesecke, A., Lüddecke, T.O.D., Hafer, R.F. and Morris, D.J. (1999). Viscosity measurements of ammonia, R32, R134a. Vapor buoyancy and radial acceleration in capillary viscometers. Int. J. Thermophysics 20: 401-434.

Lemmon, E.W. and McLinden, M.O. (2001). Method for estimating mixture equation of state parameters. Thermophysical Properties and Transfer Processes of New Refrigerants, International Institute of Refrigeration, Paderborn, Germany.

Lemmon, E.W., McLinden, M.O. and Huber, M.L. (2002). NIST Standard Reference Database 23, NIST Reference Fluid Thermodynamic and Transport Properties-REFPROP, version 7.0. Standard Reference Data Program, National Institute of Standards and Technology.

Lemmon, E.W. and Jacobsen, R.T. (2001). An international standard formulation for the thermodynamic properties of 1,1,1-trifluoroethane (HFC-143a) for temperatures from 161 to $500 \mathrm{~K}$ and pressures to $50 \mathrm{MPa}$. J. Phys. Chem. Ref. Data 29: 521-552.

Lemmon, E.W. and Jacobsen, R.T. (2002a). An equation of state for pentafluoroethane (HFC$125)$ for temperatures from the triple point $(172.52 \mathrm{~K})$ to $500 \mathrm{~K}$ and pressures to $60 \mathrm{MPa} . J$. Phys. Chem. Ref. Data, (to be submitted for publication).

Lemmon, E.W. and Jacobsen, R.T. (2002b). Equations of state for mixtures of R-32, R-125, R134a, R-143a, and R-152a. J. Phys. Chem. Ref. Data, (to be submitted for publication).

Lísal, M. and Vacek, V. (1996). Effective potentials for liquid simulation of the alternative refrigerants HFC-32: $\mathrm{CH}_{2} \mathrm{~F}_{2}$ and HFC-23: $\mathrm{CHF}_{3}$. Fluid Phase Equilibria 118: 61-76.

Magee, J.W. (1996). Isochoric p- $\rho$-T measurements on difluoromethane (R32) from 142 to $396 \mathrm{~K}$ and pentafluoroethane (R125) from 178 to $398 \mathrm{~K}$ at pressures to $35 \mathrm{MPa}$. Int. J. Thermophysics 17: 803-822.

Magee, J.W., Deal, R.J. and Blanco, J.C. (1998). High-temperature adiabatic calorimeter for constant-volume heat capacity measurements of compressed gases and liquids. J. Res. NIST 103: 63-75. 
McLinden, M.O., Lemmon, E.W. and Jacobsen, R.T. (1998). Thermodynamic properties for the alternative refrigerants. Int. J. Refrigeration 21: 322-338.

McLinden, M.O., Klein, S.A. and Perkins, R.A. (2000). An extended corresponding states model for the thermal conductivity of refrigerants and refrigerant mixtures. Int. J. Refrigeration 23: 43-63.

Nabizadeh, H. and Mayinger, F. (1999). Viscosity of gaseous R404A, R407C, R410A, and R507. Int. J. Thermophysics 20: 777-790.

Outcalt, S.L. and McLinden, M.O. (1996). A modified Benedict-Webb-Rubin equation of state for the thermodynamic properties of R152a (1,1-difluoroethane). J. Phys. Chem. Ref. Data 25: 605-636.

Perkins, R.A., Roder, H.M. and Nieto de Castro, C.A. (1991). A high-temperature transient hotwire thermal conductivity apparatus for fluids. J. Res. NIST 96: 247-269.

Ripple, D. and Defibaugh, D.R. (1997). Viscosity of the saturated liquid phase of three fluorinated ethanes: R152a, R143a, and R125. J. Chem. Eng. Data 42: 360-364.

Roder, H.M., Perkins, R.A., Laesecke, A. and Nieto de Castro, C. (2000). Absolute steady-state thermal conductivity measurements by use of a transient hot-wire system. J. Res. NIST 105: 221-253.

Tanaka, Y. and Sotani, T. (1998). Survey of viscosity data for HFC refrigerants and their mixtures (revised). Kobe University, Report to IEA Annex 18.

Tillner-Roth, R. and Baehr, H.D. (1994). An international standard formulation of the thermodynamic properties of 1,1,1,2-tetrafluoroethane (HFC-134a) covering temperatures from $170 \mathrm{~K}$ to $455 \mathrm{~K}$ at pressures up to $70 \mathrm{MPa}$. J. Phys. Chem. Ref. Data 23: 657-729.

Tillner-Roth, R. and Yokozeki, A. (1997). An international standard equation of state for difluoromethane (R-32) for temperatures from the triple point at $136.34 \mathrm{~K}$ to $435 \mathrm{~K}$ and pressures up to $70 \mathrm{MPa}$. J. Phys. Chem. Ref. Data 26: 1273-1328.

Yata, J. (1998). A data survey of the thermal conductivity for HFC mixtures (revised). Kyoto Institute of Technology, Report to IEA Annex 18.

Yokoyama, C., Nishino, T. and Takahashi, M. (2001). Viscosity of gaseous mixtures of HFC125 + HFC-32. Int. J. Thermophysics 22: 1329-1347. 